NEUTRAL PION ELECTROPRODUCTION IN THE $\triangle$ RESONANCE REGION

\author{
By \\ Anthony Nicholas Villano \\ A Thesis Submitted to the Graduate \\ Faculty of Rensselaer Polytechnic Institute \\ in Partial Fulfillment of the \\ Requirements for the Degree of \\ DOCTOR OF PHILOSOPHY \\ Major Subject: Physics
}

Approved by the

Examining Committee:

Paul Stoler, Thesis Adviser

Gary Adams, Member

Robert Block, Member

Peter Bosted, Member

Mark Jones, Member

Jim Napolitano , Member

Rensselaer Polytechnic Institute

Troy, New York

November 2007

(For Graduation December 2007) 


\title{
NEUTRAL PION ELECTROPRODUCTION IN THE $\Delta$ RESONANCE REGION
}

\author{
By \\ Anthony Nicholas Villano \\ An Abstract of a Thesis Submitted to the Graduate \\ Faculty of Rensselaer Polytechnic Institute \\ in Partial Fulfillment of the \\ Requirements for the Degree of \\ DOCTOR OF PHILOSOPHY \\ Major Subject: Physics \\ The original of the complete thesis is on file \\ in the Rensselaer Polytechnic Institute Library
}

\footnotetext{
Examining Committee:

Paul Stoler, Thesis Adviser

Gary Adams, Member

Robert Block, Member

Peter Bosted, Member

Mark Jones, Member

Jim Napolitano , Member
}

Rensselaer Polytechnic Institute

Troy, New York

November 2007

(For Graduation December 2007) 
(C) Copyright 2007

by

Anthony Nicholas Villano

All Rights Reserved 


\section{ACKNOWLEDGEMENTS}

A spark of interest in science is a wonderful thing to experience and equally wonderful is the realization that you can help the scientific community to discover new and amazing facts about our world. These simple realizations alone cannot, however, grow into a developed scientist. There are many people who help in supporting and

maturing a scientists interests through their life. Without these people I certainly could not have contributed to the scientific community.

My family and my friends were instrumental throughout my years at RPI since they were always there for me when I went home, even if I was only able to escape for a while. Whats more is that I found that I had many homes when I was able to visit my friends and family in many different cities. I am truly greatful for those opportunities and all the time we spent together at family gatherings or spontaneous weekends away from school.

I am lucky to have many different relationships in my academic career, each which has enriched me as a scientist and changed the way I look at physics to some extent. Gary Adams was very helpful when I first entered graduate school and wanted to do research with the particle physics group at RPI. During my research at Jefferson Lab I have had the benefit of the experience of Peter Bosted, Valery Koubarovsky, and Mark Jones who shaped my understanding of the physics probed at Jefferson Lab and indeed experimental physics as a whole. Several other members of the Hall C and Hall B collaborations were very helpful throughout my graduate experience. I was also lucky to have the benefit of our collaborator at the University of Witwatersrand, Simon Connell. Back at RPI, I benefited tremendously from the Teaching experiences and physics discussions with Jim Napolitano. I learned much from our conversations on various topics in physics and hope to take some of what Jim taught me and try to forge a deeper understanding of our world. During my time at RPI John Cummings was also instrumental in my development. I realized very quickly that I could pick physics conversations with Jim and John on a pretty regular basis and was happy at all the things we could learn together. Some of the 
best times I've had in physics so far took place while scrawling different equations and diagrams on the chalk boards in their offices as we argued about many things. I truly miss our conversations and am happy to have these fellows as friends.

I would also like to mention my thesis advisor, Paul Stoler. I have learned a great deal from him and am very proud to have been his student. I was lucky to begin work with Paul during my undergraduate years and continue through graduate school. I find that as an advisor he showed me a perfect mixture of motivation, freedom, and advice. I am happy to work with Paul in a physics setting and also to have his friendship.

There are also several people I would like to mention who contributed to my general well-being during graduate school whether it be in a collaborative effort or during fun grad student outings. I had the benefit of working with Mark Dalton and Georgie Mbianda from the University of Witwatersrand and also had fun with these guys during several meetings at Jefferson Lab. At RPI, I had some fun times with Johnny Goett, Puneet Khetarpal, Balazs Kozma, Matt Martin, Dave Poxson, Phil Shemella and Mike Popowich. I especially realized how fun these times were after I had moved away and came back for various events like my thesis defense and graduation.

I would like to mention the older generation in my family without whom I could have never accomplished anything in life. Aunt Carmella, Aunt Arlene, Uncle Joe, Uncle Frank, Grandpa, Papa, and Grandma were all extremely supportive in everything I did and certainly encouraged me to complete my schooling.

Finally, I will mention my mother who always pushed me to do the right thing and taught me by example to never give up. I hope that I can grow into as respectable and strong of a person as she is. 


\section{CONTENTS}

LIST OF TABLES $\ldots \ldots \ldots \ldots \ldots \ldots \ldots$ vii

LIST OF FIGURES . . . . . . . . . . . . . . . . . viii

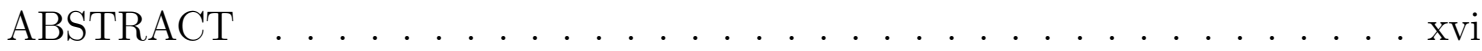

1. Hadronic Physics . . . . . . . . . . . . . . . . . . . . 1

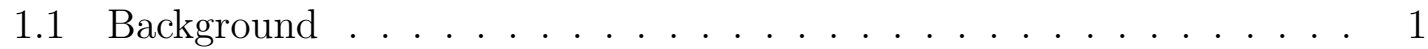

1.2 Elastic scattering off of extended particles $\ldots \ldots \ldots \ldots$

1.3 Inelastic Scattering off of extended particles . . . . . . . . . 5

1.4 Resonance Production _. . . . . . . . . . . . . . . . . 9

1.4.1 Excitation of Spin- $\frac{3}{2}$ State $\ldots \ldots \ldots \ldots$

1.5 Multipole Amplitudes . . . . . . . . . . . . . . . . . . . . . 12

1.5.1 Electromagnetic Transition . . . . . . . . . . . . . . . . 13

1.5.2 Helicity Amplitudes . . . . . . . . . . . . . . . . . . 14

1.5.3 CGLN Amplitudes . . . . . . . . . . . . . . . . 16

1.6 Previous Measurements and Thrust . . . . . . . . . . . . . . 19

1.6.1 pQCD Expectations . . . . . . . . . . . . . 24

1.6.2 Previous World Data . . . . . . . . . . . . . . 25

2. Kinematics . . . . . . . . . . . . . . . . . . . . . . . . . . . . 29

2.1 Kinematics Before Dynamics . . . . . . . . . . . . . . . . . . 29

2.2 Production of $\pi^{0}$ Mesons . . . . . . . . . . . . . . . . . . . . 29

2.2.1 Density Matrix and Rotations . . . . . . . . . . . . . 40

2.2 .2 Symmetry and Cross Sections . . . . . . . . . . . . 42

2.3 Elastic Radiative . . . . . . . . . . . . . . . . . . . . 46

2.4 Meson Production Radiative . . . . . . . . . . . . . . . . . . . . . . . 49

3. Theoretical Issues . . . . . . . . . . . . . . . . . . . . . . . 52

3.1 Theory Meets the Strong . . . . . . . . . . . . . . . . 52

3.2 Quark Model . . . . . . . . . . . . . . . . . . . 52

3.3 pQCD Predictions $\ldots \ldots \ldots \ldots \ldots \ldots \ldots \ldots$

3.4 Phenomenological Analysis . . . . . . . . . . . . . . . . . 60 
4. Dynamics of Physics Backgrounds . . . . . . . . . . . . . . 62

4.1 Backgrounds and Interferences . . . . . . . . . . . . . . . . . . 62

4.2 Elastic Radiative . . . . . . . . . . . . . . . . . . 63

4.3 Meson Radiative . . . . . . . . . . . . . . . . . . . . 69

4.4 Incoherent Radiation and Straggling . . . . . . . . . . . . . . 73

4.5 Multiple Scattering . . . . . . . . . . . . . . . . . . . . . 74

5. Spectrometers and Electronics . . . . . . . . . . . . . . 75

5.1 Overview . . . . . . . . . . . . . . . . . . 75

5.2 Beamline........................... 75

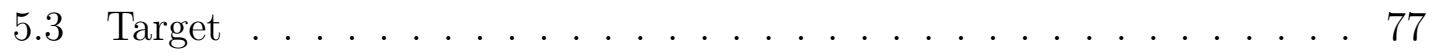

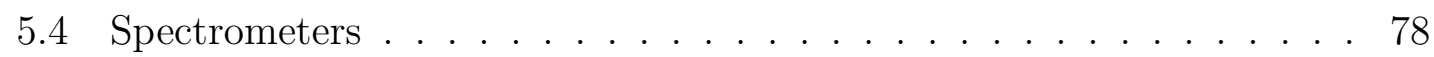

5.5 Electronics and Data Acquisition . . . . . . . . . . . . . . . . 84

6. Calibration and Efficiency . . . . . . . . . . . . . . 87

6.1 Absolute Measurements . . . . . . . . . . . . . . . . . 87

6.2 Calorimeters . . . . . . . . . . . . . . . . . 87

6.3 Drift Chambers . . . . . . . . . . . . . . . . . 88

6.4 Target Boiling . . . . . . . . . . . . . . . . . . . 88

6.5 Proton Absorption . . . . . . . . . . . . . . . . . . . . 92

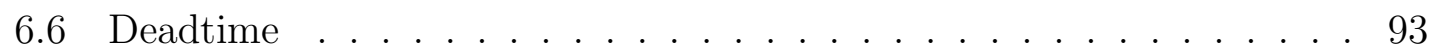

7. Experimental Corrections . . . . . . . . . . . . . . . . . 97

7.1 Realistic Measurements . . . . . . . . . . . . . . 97

7.2 Data Exclusion . . . . . . . . . . . . . . . . . . . . . 98

7.2.1 Collimator Functions . . . . . . . . . . . . . . . 101

7.2.2 SOS Collimator .................... 101

7.2.3 HMS Collimator . . . . . . . . . . . . . . . . . . 102

7.3 Fiducial and PID Rejection Analysis . . . . . . . . . . . . . 103

7.3.1 Time of Flight . . . . . . . . . . . . . . . . . 103

7.3.2 Missing Mass . . . . . . . . . . . . . . . . . 109

7.3.3 Electron Calorimeter . . . . . . . . . . . . . . . . . 113

7.3.4 Electron Čerenkov . . . . . . . . . . . . . . . . . 114

7.4 Apparatus Backgrounds . . . . . . . . . . . . . 116

7.4.1 Accidental Counts . . . . . . . . . . . . . . 117 
7.4.2 Target End Cap Counts . . . . . . . . . . . . . . . . . . 119

7.5 Elastic Radiative Corrections . . . . . . . . . . . . . . . . . . . . . 121

7.5.1 Elastic Radiative Data Removal . . . . . . . . . . . . . . . 122

8. Observable Extraction . . . . . . . . . . . . . . . . . . . 130

8.1 Observables from Experiments . . . . . . . . . . . . . . 130

8.2 Invariants in the Lab . . . . . . . . . . . . . . . . . . 130

8.3 Detector Acceptance . . . . . . . . . . . . . . . . . . . 136

8.3.1 General Acceptance . . . . . . . . . . . . . . . . . 136

8.3.2 Moving Spectrometer Acceptance . . . . . . . . . . . . 138

8.4 Monte Carlo Simulation . . . . . . . . . . . . . . . . . . . 139

8.5 Cross Section Extraction . . . . . . . . . . . . . . . . . . 141

8.6 Kinematic Bin Centering . . . . . . . . . . . . . . . . . . . . 144

8.7 Full Analysis Procedure . . . . . . . . . . . . . . . . . . 145

9. Consistency Checks for Cross Sections _ . . . . . . . . . . . 152

9.1 Data Checks . . . . . . . . . . . . . . . . . . . . . . 152

9.2 Inclusive Check . . . . . . . . . . . . . . . . . . . . 152

9.3 Setting Overlap Check . . . . . . . . . . . . . . . . . . 157

9.4 SOS acceptance issues . . . . . . . . . . . . . . . . . 161

10. Results and Systematic Errors _. . . . . . . . . . . . . . . . 163

10.1 The Tie That Binds . . . . . . . . . . . . . . . . 163

10.2 Systematic Errors . . . . . . . . . . . . . . . 163

10.3 Physical Extractions . . . . . . . . . . . . . . . 166

10.3.1 Multipole Ratios . . . . . . . . . . . . . . . 170

10.3.2 Transition Form Factors _ . . . . . . . . . . . . . 173

10.4 Systematic Studies . . . . . . . . . . . . . 176

10.4.1 Missing Mass Restriction . . . . . . . . . . . . . 176

10.4.2 Elastic Radiative Rejection . . . . . . . . . . . . . . 178

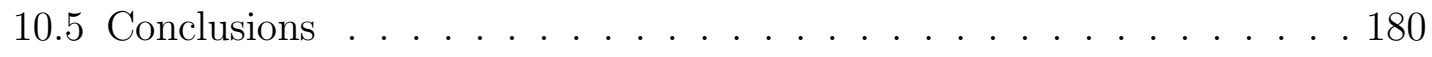
APPENDICES 
A. Leptonic Currents and Couplings . . . . . . . . . . . . . . . . 191

A.1 Dirac Bilinears . . . . . . . . . . . . . . . . . 191

A.2 Dirac Equation and Lagrangian . . . . . . . . . . . . . . . . 192

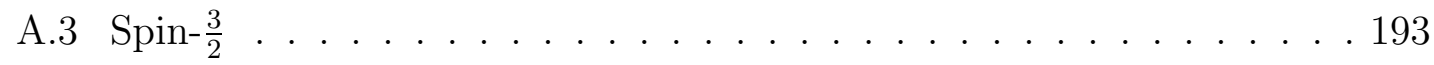

B. Lengthy Derivations . . . . . . . . . . . . . . . . 195

B.1 Inclusive Lepton Scattering . . . . . . . . . . . . . . . 195

B.2 Electromagnetic Transition . . . . . . . . . . . . . . . . 198

C. Kinematical Conventions . . . . . . . . . . . . . . . . . . 209

C.1 Introduction . . . . . . . . . . . . . . . . . . 209

C.2 Elastic Scattering . . . . . . . . . . . . . . . . 209

C.3 Pion Production . . . . . . . . . . . . . . . . . . . 211

C.4 Elastic Radiative . . . . . . . . . . . . . . . . . . . . 212

C.5 Pion Production Radiative . . . . . . . . . . . . . . . 213

D. Experimental Parameters . . . . . . . . . . . . . . . . . . . . 214

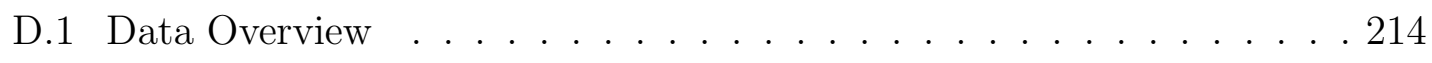

D.2 Experimental Settings . . . . . . . . . . . . . 216

E. Experimental Data . . . . . . . . . . . . . . . . 218

E.1 Differential Cross Sections . . . . . . . . . . . . 218 


\section{LIST OF TABLES}

1.1 Reaction parameters. . . . . . . . . . . . . . . 19

3.1 Quark Charges. .................... 54

$5.1 \quad \mathrm{LH}_{2}$ target wall thickness. . . . . . . . . . . . . . . . 78

5.2 HMS utilized detector components. . . . . . . . . . . . 78

5.3 SOS utilized detector components. . . . . . . . . . . . . 79

6.1 Target rate statistics. $\theta_{\text {sos }}=47.5^{\circ}$ and $p_{\text {sos }}=1.74 \mathrm{GeV}$. . . . . . . . 90

6.2 Target rate statistics. $\theta_{\text {sos }}=69.9^{\circ}$ and $p_{\text {sos }}=1.04 \mathrm{GeV}$. . . . . . . . . 92

7.1 Variable Names. . . . . . . . . . . . . . . . . . . . . . . . . . . 99

7.2 Projected positions. . . . . . . . . . . . . . . . 101

7.3 Missing mass resolutions. . . . . . . . . . . . . . . . . . . . 112

7.4 Radiative rejection in different $\cos \theta^{*}$ bins. . . . . . . . . . . . . 127

8.1 $Q^{2}$ values for each invariant energy bin. Invariant energy and $Q^{2}$ are not independent but are kinematically correlated. . . . . . . . . . . . 135

8.2 E01-002 analysis binning for low $Q^{2}$ data. . . . . . . . . . . 146

8.3 E01-002 analysis binning for high $Q^{2}$ data. . . . . . . . . . . . 146

$9.1 \quad \chi^{2}$ values for pass comparison. . . . . . . . . . . . . . . 161

10.1 Systematic errors for E01-002 extracted cross sections. The size reported for is the final estimated error on the experimental result. . . . . 165

10.2 Efficiency corrections for E01-002 extracted cross sections. The size reported for is the size (or average size) of the correction factor applied to the data. . . . . . . . . . . . . . . . . . . 166

A.1 Dirac bilinears. . . . . . . . . . . . . . . . . . 192

D.1 Experimental spectrometer settings for $Q^{2}=6.36 \mathrm{GeV}^{2} \ldots$. . . . . . 214

D.2 Experimental spectrometer settings for $Q^{2}=7.7 \mathrm{GeV}^{2}$. . . . . . . . 214

D.3 HMS magnet settings. . . . . . . . . . . . . . . . . . 217

D.4 SOS magnet settings. . . . . . . . . . . . . . . . . 217 


\section{LIST OF FIGURES}

1.1 Generalized hadronic vertex. . . . . . . . . . . . . . . . 2

1.2 Lab frame elastic scattering. . . . . . . . . . . . . . . . . 4

1.3 Generalized inelastic scattering. . . . . . . . . . . . . . . 5

1.4 Lab frame inclusive elastic scattering. . . . . . . . . . . . . . 7

1.5 Lepton scattering excitation of an asymptotic $\Delta . \ldots . . . . . . .10$

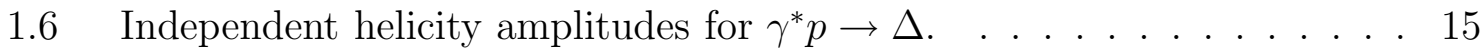

1.7 Current experimental situation for $R_{E M}$. The points are separated by laboratory or detector. The outer error bars are the sum (in quadrature) of the statistical and systematic errors. . . . . . . . . . . . 26

1.8 Current experimental situation for $R_{S M}$. The points are separated by laboratory or detector. The outer error bars are the sum (in quadrature) of the statistical and systematic errors. . . . . . . . . . . . 26

1.9 Current experimental situation for $G_{M}^{*}$ in the Jones and Scadron convention [52]. The points are separated by primary investigator. . . . . . 27

2.1 Lab frame neutral pion production. . . . . . . . . . . . . . 30

2.2 Center of mass frame neutral pion production. . . . . . . . . . . 33

2.3 Kinematics of elastic radiative process. . . . . . . . . . . . . . 47

2.4 True momentum transfer to hadronic current. . . . . . . . . . . . . 48

2.5 Elastic radiative events and their projections onto the pion kinematics. 50

2.6 Kinematics of radiative meson production process. . . . . . . . . 51

3.1 pQCD hard transition amplitude for $\Delta$ excitation. . . . . . . . . . . 59

4.1 Leading order elastic electron proton scattering. . . . . . . . . . . . 65

$4.2 \mathcal{O}\left(\alpha^{\frac{3}{2}}\right)$ amplitude corrections. . . . . . . . . . . . . . . . . . 66

$4.3 \mathcal{O}\left(\alpha^{2}\right)$ amplitude corrections. . . . . . . . . . . . . . . . 67

4.4 Leading order meson production. . . . . . . . . . . . . . . . 71

$4.5 \mathcal{O}\left(\alpha^{\frac{3}{2}}\right)$ amplitude corrections for single meson production. . . . . . . . . 72 
4.6 $\mathcal{O}\left(\alpha^{2}\right)$ amplitude corrections for single meson production. . . . . . . . . 72

5.1 Top view of Hall C. The graphic was inspired by the publication of Armstrong $[5,6]$ and is not to scale. . . . . . . . . . . . . . . 80

5.2 Spectrometer coordinate systems. . . . . . . . . . . . . . 81

5.3 Collimator dimensions. . . . . . . . . . . . . . . . . . 81

5.4 Schematic view of each spectrometer. The figure is not to scale. The label "Q" is for quadrupole, "D" is for dipole and "FP" represents the focal plane. . . . . . . . . . . . . . . . . . . 82

5.5 Typical spectrometer detector package. The graphic was inspired by the publication of Armstrong [5,6]. A sample particle trajectory is shown with drift chamber hits. . . . . . . . . . . . . . . . . 83

5.6 Simple representation of the electronics and data acquisition system for experiment E01-002. . . . . . . . . . . . . . . . 85

6.1 SOS single arm rates vs. current. $\theta_{\text {sos }}=47.5^{\circ}$ and $p_{\text {sos }}=1.74 \mathrm{GeV}$. . 89

6.2 SOS single arm scaled rates vs. current. $\theta_{\text {sos }}=47.5^{\circ}$ and $p_{\text {sos }}=1.74$ GeV. . . . . . . . . . . . . . . . . . . . 89

6.3 SOS single arm rates vs. current. $\theta_{\text {sos }}=69.9^{\circ}$ and $p_{\text {sos }}=1.04 \mathrm{GeV}$. . . 91

6.4 SOS single arm scaled rates vs. current. $\theta_{\text {sos }}=69.9^{\circ}$ and $p_{\text {sos }}=1.04$

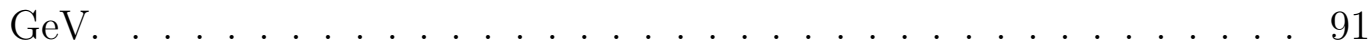

6.5 Schematic of several gate signals and times of two random events. Depending on the gate width, they can arrive within the same electronic gate. . . . . . . . . . . . . . . . . 94

6.6 Schematic of the setup to measure the electronic deadtime. . . . . . . . 95

7.1 The inverse velocities of the deuteron, proton, kaon and pion respec-

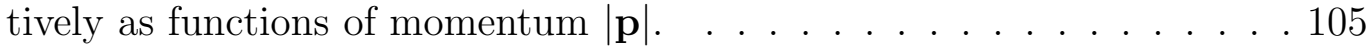

7.2 Coincidence time spectrum for proton and $\pi^{+}$at $3.10 \mathrm{GeV}$ spectrometer central momentum. . . . . . . . . . . . . . . . . . . 106

7.3 $t_{c}^{\star}$ vs. hadron momentum for coincidence data normalized to make the proton vertical. . . . . . . . . . . . . . . . . 107

$7.4 \tilde{t}_{c}$ vs. hadron momentum for coincidence data normalized to make the pion vertical. 
7.5 $t_{c}^{\star}$ for coincidence data normalized to make the proton peak sharp. The structures visible on the low and high edges of the plot are due to the coincidence events from different RF beam bunches. . . . . . . . . . . 108

7.6 $\tilde{t}_{c}$ for coincidence data normalized to make the pion peak sharp. . . . . 109

7.7 Monte Carlo simulation of $M_{x}^{2}$ and $\cos \theta_{p}^{*}$. The vertical lines in the two-dimensional plot signify the boundaries for each of the projections plotted in the lower portion of the figure. The projections progress from left to right and then top to bottom with increasing $\cos \theta_{p}^{*}$. The vertical lines on the projection plots mark the missing mass restrictions used in the analysis. . . . . . . . . . . . . . . . . 11

7.8 Data distribution in $M_{x}^{2}$ and $\cos \theta_{p}^{*}$. The vertical lines in the twodimensional plot signify the boundaries for each of the projections plotted in the lower portion of the figure. The projections progress from left to right and then top to bottom with increasing $\cos \theta_{p}^{*}$. The vertical lines on the projection plots mark the missing mass restrictions used in the analysis. . . . . . . . . . . . . . . . . . . . . 111

7.9 Trends of the $80 \%$ widths. . . . . . . . . . . . . . . . 112

7.10 Calorimeter electron selection cut. The shaded region corresponds to the accepted events identified as electrons. . . . . . . . . . . . 113

7.11 Čerenkov electron selection cut. The shaded region corresponds to that which is accepted into the analysis. . . . . . . . . . . . . . . 114

7.12 Čerenkov electron selection cut pedestal region. . . . . . . . . . 115

7.13 Remaining calorimeter contamination. . . . . . . . . . . 115

7.14 Remaining calorimeter contamination with other particle identifications in place. . . . . . . . . . . . . . . . . 116

7.15 Timing spectrum of coincidence events. The shaded insert shows the typical analysis cut made on this spectrum. . . . . . . . . . . . 118

7.16 Timing spectrum of coincidence events. The shaded insert shows the wing of the timing distribution. . . . . . . . . . . . . . . 118

7.17 Target window event geometry. . . . . . . . . . . . . . 120

7.18 Target window counts from the SOS. . . . . . . . . . . . . 121

7.19 Target window counts. . . . . . . . . . . . . . 121

$7.20 M_{x}^{2}$ vs. $\phi^{*}$ with only standard cuts and $W>1.1 \mathrm{GeV}$ applied. . . . . . 123 
$7.21 \phi^{*}$ vs. $\cos \theta^{*}$ with only standard cuts and $W>1.1 \mathrm{GeV}$ applied. . . . 123

$7.22 X_{\Omega}$ vs. $Y_{\Omega}$ with only standard cuts and $W>1.1 \mathrm{GeV}$ applied. . . . . 124

7.23 Cosine of the angle of emitted radiation and a displayed possible rejection area. . . . . . . . . . . . . . . . . . 125

7.24 $X_{\Omega}$ vs. $Y_{\Omega}$ with restriction on radiation angle and $W>1.1 \mathrm{GeV}$ applied. 125

$7.25 M_{x}^{2}$ vs. $\phi^{*}$ with $W>1.1 \mathrm{GeV}$ and a possible elastic rejection superim-

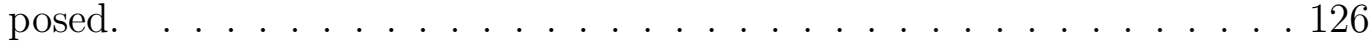

7.26 Radiative rejection overlay for lowest $\cos \theta^{*}$ bin. . . . . . . . . . . 127

7.27 Radiative rejection overlay for intermediate $\cos \theta^{*}$ bin. . . . . . . . 128

7.28 Radiative rejection overlay for highest $\cos \theta^{*}$ bin. . . . . . . . . . 128

$7.29 X_{\Omega}$ vs. $Y_{\Omega}$ with radiative cut and $W>1.1 \mathrm{GeV}$ applied. . . . . . . 129

8.1 Lab frame quantities for $W$ contours in $0.1 \mathrm{GeV}$ step increments running from $Q^{2}$ of $5.0 \mathrm{GeV}^{2}$ to $9.0 \mathrm{GeV}^{2}$ for $E=5.5 \mathrm{GeV}$. . . . . . . . 132

8.2 Lab frame hadronic kinematics. . . . . . . . . . . . . . . . . . . 132

8.3 $W$ contours for planar kinematics in the laboratory variables for the outgoing nucleon. The contours are calculated for $\theta_{e}=47.5^{\circ}$ and the boxes represent the HMS settings from this experiment. . . . . . . . 134

8.4 $W$ contours for planar kinematics in the laboratory variables for the outgoing nucleon. The contours are calculated for $\theta_{e}=69.9^{\circ}$ and the boxes represent the HMS settings from this experiment. . . . . . . 135

8.5 Typical binning situation. . . . . . . . . . . . . . . . . . . . . . 144

8.6 Radiative corrections for $W=1.232 \mathrm{GeV}$ and $Q^{2}=6.36 \mathrm{GeV}^{2}$. The inelasticity parameter $v$ was varied to produce several curves. The shift of the inelasticity parameter from nominal, $\delta v_{c u t}$ is displayed in the figure. The value of $\cos \theta^{*}$ is 0.0 here. . . . . . . . . . . . 149

8.7 Radiative corrections for $W=1.232 \mathrm{GeV}$ and $Q^{2}=6.36 \mathrm{GeV}^{2}$. The inelasticity parameter $v$ was varied to produce several curves. The shift of the inelasticity parameter from nominal, $\delta v_{c u t}$ is displayed in the figure. The value of $\phi^{*}$ is 0.0 here. . . . . . . . . . . . . . 149

8.8 Flow chart describing analysis procedure. . . . . . . . . . . . 151

9.1 Comparison to inclusive cross section at $Q^{2}=6.36 \ldots \ldots \ldots$

$9.2 \quad$ Ratio to inclusive cross section at $Q^{2}=6.36 \ldots \ldots \ldots \ldots$ 
9.3 Overlap pass 1 for $Q^{2}=6.36$ SOS setting. . . . . . . . . . . 158

9.4 Overlap pass 2 for $Q^{2}=6.36$ SOS setting. . . . . . . . . . . 159

9.5 Comparison of non radiatively corrected differential cross sections at $W=1.232 \mathrm{GeV}$ and $Q^{2}=6.36 \mathrm{GeV}^{2} \ldots \ldots \ldots \ldots$

9.6 Comparison of non radiatively corrected differential cross sections at $W=1.232 \mathrm{GeV}$ and $Q^{2}=6.36 \mathrm{GeV}^{2} \ldots \ldots \ldots \ldots 1$

10.1 Fit to differential cross sections at $W=1.232 \mathrm{GeV}$ and $Q^{2}=6.36 \mathrm{GeV}^{2} .169$

10.2 Fit to differential cross sections at $W=1.232 \mathrm{GeV}$ and $Q^{2}=6.36$ $\mathrm{GeV}^{2}$. The data is binned in $\cos \theta^{*}$ and displayed as a function of $\phi^{*}$. . 170

10.3 Results of the simple $M_{1+}$ dominance fit for the lower $Q^{2}$ data set as a function of invariant energy $W \ldots \ldots \ldots$. . . . . . . . . . . . . .

10.4 Results of the simple $M_{1+}$ dominance fit for the higher $Q^{2}$ data set as a function of invariant energy $W \ldots \ldots \ldots . \ldots . \ldots 173$

10.5 The total angle integrated cross section for lower $Q^{2}$ settings fit with a Breit-Wigner function and a fractional power polynomial of second order. . . . . . . . . . . . . . . . . . . . . 174

10.6 The total angle integrated cross section for higher $Q^{2}$ settings fit with a Breit-Wigner function and a polynomial of second order. The dark inverted triangle data points are the only ones used to constrain the fit due to lack of statistics for higher $W$ and the possibility of small elastic radiative contamination at lower $W \ldots \ldots \ldots$. . . . . . . . . . . .

10.7 Extracted value of the Jones and Scadron $G_{M}^{*}$ transition form factor. The values include this and several other exclusive analyses. . . . . . 175

10.8 Various missing mass restrictions used to probe the systematic error of the nominal width. The highlighted lines represent the nominal width. 177

10.9 The variation of the $R_{E M}$ estimator on missing mass restriction. From left to right the restrictions are labeled from narrowest to widest with the arrow marking the nominal. . . . . . . . . . . . . . . 178

10.10 Radiative rejection overlay for lowest $\cos \theta^{*}$ bin. Several variations on the nominal rejection curves are displayed. The nominal curves appear as highlighted.

10.11 Radiative rejection overlay for intermediate $\cos \theta^{*}$ bin. Several variations on the nominal rejection curves are displayed. The nominal curves appear as highlighted. 
B.1 Radiative transition in multipole basis. . . . . . . . . . . . . . 199

B.2 Generalized electromagnetic transition in multipole basis. . . . . . . . . 199

C.1 Angular definitions. . . . . . . . . . . . . . . . . . 210

D.1 Missing mass distribution mapped onto $\mathrm{W}$ distribution. . . . . . . . 215

D.2 Invariant mass vs. missing mass distribution. . . . . . . . . . . 216

E.1 Center of mass differential cross sections for $W=1.112 \mathrm{GeV}$ and $Q^{2}=$ $6.564 \mathrm{GeV}^{2} \cdot \frac{\chi^{2}}{n_{\text {dof }}}=1.145 . \quad \ldots \ldots \ldots . \ldots \ldots 219$

E.2 Center of mass differential cross sections for $W=1.112 \mathrm{GeV}$ and $Q^{2}=$ $6.564 \mathrm{GeV}^{2} \cdot \frac{\chi^{2}}{n_{\text {dof }}}=1.145$.

E.3 Center of mass differential cross sections for $W=1.152 \mathrm{GeV}$ and $Q^{2}=$ $6.5 \mathrm{GeV}^{2} \cdot \frac{\chi^{2}}{n_{d o f}}=1.082$.

E.4 Center of mass differential cross sections for $W=1.152 \mathrm{GeV}$ and $Q^{2}=$ $6.5 \mathrm{GeV}^{2} \cdot \frac{\chi^{2}}{n_{d o f}}=1.082$.

E.5 Center of mass differential cross sections for $W=1.192 \mathrm{GeV}$ and $Q^{2}=$ $6.432 \mathrm{GeV}^{2} \cdot \frac{\chi^{2}}{n_{\text {dof }}}=1.279$.

E.6 Center of mass differential cross sections for $W=1.192 \mathrm{GeV}$ and $Q^{2}=$ $6.432 \mathrm{GeV}^{2} \cdot \frac{\chi^{2}}{n_{\text {dof }}}=1.279$.

E.7 Center of mass differential cross sections for $W=1.232 \mathrm{GeV}$ and $Q^{2}=$

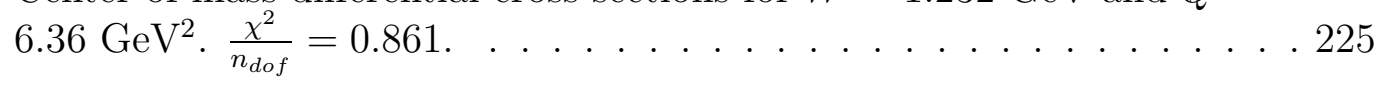

E.8 Center of mass differential cross sections for $W=1.232 \mathrm{GeV}$ and $Q^{2}=$

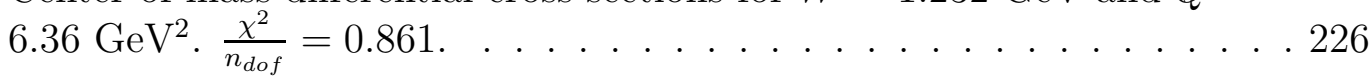

E.9 Center of mass differential cross sections for $W=1.272 \mathrm{GeV}$ and $Q^{2}=$ $6.288 \mathrm{GeV}^{2} \cdot \frac{\chi^{2}}{n_{\text {dof }}}=1.795$.

E.10 Center of mass differential cross sections for $W=1.272 \mathrm{GeV}$ and $Q^{2}=$

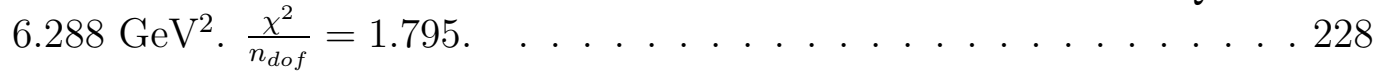

E.11 Center of mass differential cross sections for $W=1.312 \mathrm{GeV}$ and $Q^{2}=$ $6.212 \mathrm{GeV}^{2} \cdot \frac{\chi^{2}}{n_{\text {dof }}}=1.595$.

E.12 Center of mass differential cross sections for $W=1.312 \mathrm{GeV}$ and $Q^{2}=$ $6.212 \mathrm{GeV}^{2} \cdot \frac{\chi^{2}}{n_{\text {dof }}}=1.595$. 
E.13 Center of mass differential cross sections for $W=1.352 \mathrm{GeV}$ and $Q^{2}=$ $6.136 \mathrm{GeV}^{2} \cdot \frac{\chi^{2}}{n_{d o f}}=2.185$.

E.14 Center of mass differential cross sections for $W=1.352 \mathrm{GeV}$ and $Q^{2}=$ $6.136 \mathrm{GeV}^{2} \cdot \frac{\chi^{2}}{n_{\text {dof }}}=2.185$.

E.15 Center of mass differential cross sections for $W=1.392 \mathrm{GeV}$ and $Q^{2}=$

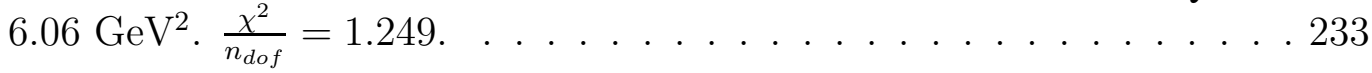

E.16 Center of mass differential cross sections for $W=1.392 \mathrm{GeV}$ and $Q^{2}=$ $6.06 \mathrm{GeV}^{2} \cdot \frac{\chi^{2}}{n_{\text {dof }}}=1.249 . \ldots \ldots \ldots \ldots \ldots \ldots \ldots \ldots \ldots . \ldots \ldots 234$

E.17 Center of mass differential cross sections for $W=1.112 \mathrm{GeV}$ and $Q^{2}=$ $7.924 \mathrm{GeV}^{2} \cdot \frac{\chi^{2}}{n_{\text {dof }}}=0.645$.

E.18 Center of mass differential cross sections for $W=1.112 \mathrm{GeV}$ and $Q^{2}=$ $7.924 \mathrm{GeV}^{2} \cdot \frac{\chi^{2}}{n_{\text {dof }}}=0.645$.

E.19 Center of mass differential cross sections for $W=1.152 \mathrm{GeV}$ and $Q^{2}=$ $7.848 \mathrm{GeV}^{2} \cdot \frac{\chi^{2}}{n_{d o f}}=0.642$.

E.20 Center of mass differential cross sections for $W=1.152 \mathrm{GeV}$ and $Q^{2}=$ $7.848 \mathrm{GeV}^{2} \cdot \frac{\chi^{2}}{n_{\text {dof }}}=0.642$.

E.21 Center of mass differential cross sections for $W=1.192 \mathrm{GeV}$ and $Q^{2}=$ $7.772 \mathrm{GeV}^{2} \cdot \frac{\chi^{2}}{n_{\text {dof }}}=0.583$

E.22 Center of mass differential cross sections for $W=1.192 \mathrm{GeV}$ and $Q^{2}=$ $7.772 \mathrm{GeV}^{2} \cdot \frac{\chi^{2}}{n_{\text {dof }}}=0.583$.

E.23 Center of mass differential cross sections for $W=1.232 \mathrm{GeV}$ and $Q^{2}=$ $7.692 \mathrm{GeV}^{2} \cdot \frac{\chi^{2}}{n_{\text {dof }}}=0.785$.

E.24 Center of mass differential cross sections for $W=1.232 \mathrm{GeV}$ and $Q^{2}=$ $7.692 \mathrm{GeV}^{2} \cdot \frac{\chi^{2}}{n_{\text {dof }}}=0.785$.

E.25 Center of mass differential cross sections for $W=1.272 \mathrm{GeV}$ and $Q^{2}=$ $7.608 \mathrm{GeV}^{2} \cdot \frac{\chi^{2}}{n_{\text {dof }}}=1.435$.

E.26 Center of mass differential cross sections for $W=1.272 \mathrm{GeV}$ and $Q^{2}=$ $7.608 \mathrm{GeV}^{2} \cdot \frac{\chi^{2}}{n_{\text {dof }}}=1.435$.

E.27 Center of mass differential cross sections for $W=1.312 \mathrm{GeV}$ and $Q^{2}=$ $7.524 \mathrm{GeV}^{2} \cdot \frac{\chi^{2}}{n_{\text {dof }}}=0.737$. 
E.28 Center of mass differential cross sections for $W=1.312 \mathrm{GeV}$ and $Q^{2}=$

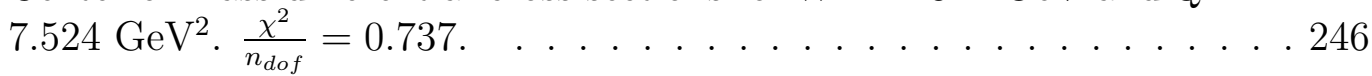

E.29 Center of mass differential cross sections for $W=1.352 \mathrm{GeV}$ and $Q^{2}=$ $7.436 \mathrm{GeV}^{2} \cdot \frac{\chi^{2}}{n_{\text {dof }}}=0.649 . \ldots \ldots \ldots \ldots 247 \ldots \ldots \ldots 2 \ldots \ldots$

E.30 Center of mass differential cross sections for $W=1.352 \mathrm{GeV}$ and $Q^{2}=$ $7.436 \mathrm{GeV}^{2} \cdot \frac{\chi^{2}}{n_{\text {dof }}}=0.649 . \ldots \ldots \ldots \ldots \ldots . \ldots \ldots 248$

E.31 Center of mass differential cross sections for $W=1.392 \mathrm{GeV}$ and $Q^{2}=$ $7.348 \mathrm{GeV}^{2} \cdot \frac{\chi^{2}}{n_{\text {dof }}}=0.442 . \ldots \ldots \ldots \ldots 24 \ldots \ldots \ldots$

E.32 Center of mass differential cross sections for $W=1.392 \mathrm{GeV}$ and $Q^{2}=$

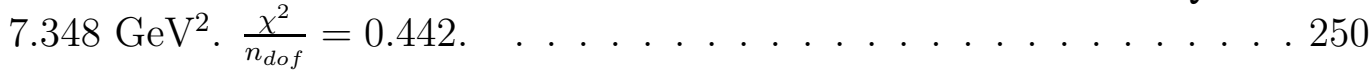




\begin{abstract}
The electroproduction of baryon resonances at high $Q^{2}$ is examined. Analysis focuses on the $\Delta(1232)$ resonance via exclusive pseudoscalar meson production of $\pi^{0}$ particles. Differential cross sections are extracted for exclusive $\pi^{0}$ electroproduction. In the central invariant mass $(W)$ region the cross sections are used to extract resonant multipole amplitudes. In particular, the ratio of the electric quadrupole to magnetic dipole amplitudes $(E 2 / M 1)$ will be discussed for the $\Delta(1232)$ resonance. The transition to pQCD is discussed in terms of $E 2 / M 1$ and other multipoles. The helicity amplitude $A_{\frac{3}{2}}$ can be used as a baryon helicity conservation meter in this context and will be discussed. The fast shrinking of the resonant contribution in the $\Delta$ region is observed at this high momentum transfer. Apart from the observables related to pQCD scaling, the transition form factor $G_{M}^{*}$ is extracted along with the scalar to magnetic dipole ratio $C 2 / M 1$.
\end{abstract}




\section{CHAPTER 1 Hadronic Physics}

\subsection{Background}

One of the most fundamental questions in physics is how the hadronic properties of our world can be described in terms of elementary degrees of freedom. Over the years a fantastic amount of work has been done to both measure the properties of hadrons and predict their behavior with greater accuracy. The result of this long period of research is what physicists have come to know as the Standard Model with its main ingredient being Quantum Chromodynamics (QCD). Unfortunately, QCD suffers from some very challenging obstacles making it very difficult to calculate physical observables in the framework of the theory. Recently there has been great progress in lattice gauge theory which gives some hope to full non-perturbative QCD calculations. Still other attempts have been made to utilize ideas of duality to calculate observables which arise in hadronic physics. Specifically, the so-called AdS/CFT correspondence has been used to link some string models to a class of quantum field theory called conformal field theories. The hope is that a string model can be found that is dual to QCD and calculable.

Luckily, despite the complexities of QCD our understanding of electroweak processes provides a wonderful probe to the hadronic world. The electroweak interaction is known in terms of a family of elementary constituents (i.e. the leptons and gauge particles $\gamma, W^{ \pm}$and $Z^{0}$ ). Since hadronic matter can, in general, carry both electromagnetic and weak charge the coupling of hadronic processes to electroweak ones provides insight into hadronic dynamics.

\subsection{Elastic scattering off of extended particles}

One of the simplest probes of the hadronic environment is elastic scattering. The cross section derivations for two elementary charged or weak-charged particles is extremely straightforward and is embodied in Mott type differential cross sections. 
When probing the hadronic environment one could expect that at low momentum ${ }^{1}$ transfer the process appears as if it were the same as the elementary process. Consider, for example, the elastic scattering of an electromagnetic current from a spin- $\frac{1}{2}$ hadron pictured in Fig. 1.1.

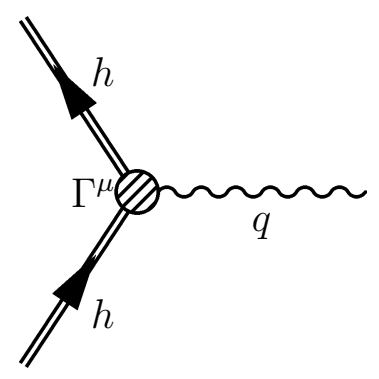

\section{Figure 1.1: Generalized hadronic vertex.}

The four-momentum $q$ represents the momentum coming from an external fermionic current (or even a real photon in which case $q^{2}=0$ ). The vertex factor $\Gamma^{\mu}$ is a generalization ${ }^{2}$ of the usual factor $\gamma^{\mu}$ which takes into account the nonelementary nature of the hadronic particle. In this way, by simply assuming that the asymptotic hadron can represented by a Dirac spinor ${ }^{3}$ all other hadronic effects are embodied in $\Gamma^{\mu}$.

Assuming that the photon originates from a leptonic current one can simply write the matrix element for the process to first order in the fine structure constant $\alpha$ as the product of two current matrix elements.

$$
\mathcal{M}=-\frac{e^{2} \eta_{\mu \nu}}{q^{2}}\left\langle h_{f}\left|J_{h a d}^{\mu}\right| h_{i}\right\rangle\left\langle l_{f}\left|J^{\nu}\right| l_{i}\right\rangle
$$

The tensor $\eta_{\mu \nu}$ is the flat Minkowski space metric tensor with the signature $(+,-,-,-)$. Since the asymptotic particles are taken to be basis states of a Fock basis constructed using tensor products of free particle eigenstates it should be understood that $\left|l_{i}\right\rangle$ and $\left|h_{i}\right\rangle$ are the initial states of the incoming lepton and hadron respectively and

\footnotetext{
${ }^{1}$ Throughout this work natural units are used where $\hbar=c=1$ and the remaining unit is chosen to be energy.

${ }^{2}$ The vertex function is allowed to be a function of kinematic Lorentz invariants for which there is exactly one in this process, $q^{2}$. Therefore we have $\Gamma^{\mu}=\Gamma^{\mu}\left(q^{2}\right)$.

${ }^{3}$ This assumption is akin to saying that the hadron is on its mass shell.
} 
similarly for the outgoing states labeled by the subscript " $f$." The factor $e$ is simply the electronic charge ${ }^{4}$. The current operators are only of the electromagnetic variety in this case and $J_{h a d}^{\mu}$ is the hadronic electromagnetic current while $J^{\nu}$ is the standard leptonic version ${ }^{5}$ (kept general for any lepton). The current matrix elements of asymptotic states are easily expanded in terms of Dirac spinors and simple matrix operators. To first order in the fine structure constant the leptonic matrix element has no "anomalous" spin magnetic moment to account for so that the matrix element takes a extremely simple form.

$$
\left\langle l_{f}\left|J^{\nu}\right| l_{i}\right\rangle=\bar{u}_{l}\left(k^{\prime}\right) \gamma^{\nu} u_{l}(k)
$$

The current matrix element for the hadronic side of the interaction should have a similar form but with $\Gamma^{\mu}\left(q^{2}\right)$ as the generalized version of $\gamma^{\mu}$. For extended (nonelementary) particles the general form of the vertex function $\Gamma^{\mu}\left(q^{2}\right)$ can be deduced from current conservation and parity symmetry. Since the system described is to interact via photon exchange with the leptonic current one can see that if the overall Lagrangian is to have parity symmetry then $\Pi^{\dagger} J_{\text {had }}^{\mu} \Pi=-J_{\text {had }}^{\mu}$, where $\Pi$ is the parity operator ${ }^{6}$. The current should also satisfy the relation $q_{\mu} J_{\text {had }}^{\mu}=0$, which is a statement of electromagnetic current conservation. It is interesting to note that electromagnetic current conservation need not be assumed, it can be proven through the requirement that the $S$ matrix be unitary to all orders [37]. The previous two facts and some properties of Dirac bilinears as seen in Section A.1 leads directly to a simple characterization of $\Gamma^{\mu}\left(q^{2}\right)$ and thus the matrix element of the hadronic current.

$$
\begin{gathered}
\left\langle h_{f}\left|J_{h a d}^{\mu}\right| h_{i}\right\rangle=\bar{u}_{h}\left(p^{\prime}\right) \Gamma^{\mu}\left(q^{2}\right) u_{h}(p) \\
\Gamma^{\mu}\left(q^{2}\right)=F_{1}\left(q^{2}\right) \gamma^{\mu}+F_{2}\left(q^{2}\right) \frac{i \sigma^{\mu \nu} q_{\nu}}{2 M_{h}}
\end{gathered}
$$

The functions $F_{1}\left(q^{2}\right)$ and $F_{2}\left(q^{2}\right)$ are known as the Dirac and Pauli form factors

\footnotetext{
${ }^{4}$ The relation to the fine structure constant is $e^{2}=4 \pi \alpha$

${ }^{5}$ For brevity of notation the symbol $J_{\text {had }}^{\mu}$ and $J^{\nu}$ are used to represent the Hilbert space current operators evaluated at the zero spacetime point $J_{\text {had }}^{\mu}(0)$ and $J^{\nu}(0)$ respectively.

${ }^{6}$ We assume the Lagrangian has a minimal coupling to the electromagnetic hadronic current of the form $A_{\mu} J^{\mu}$.
} 
respectively. These functions can parameterize any hadronic effects while having a rather simple form with regard to the interaction with electromagnetic systems.

Using the above characterization of a simple hadronic current one can calculate the scattering cross section for elastic scattering of electrons and protons in terms of the functions $F_{1}\left(q^{2}\right)$ and $F_{2}\left(q^{2}\right)$.

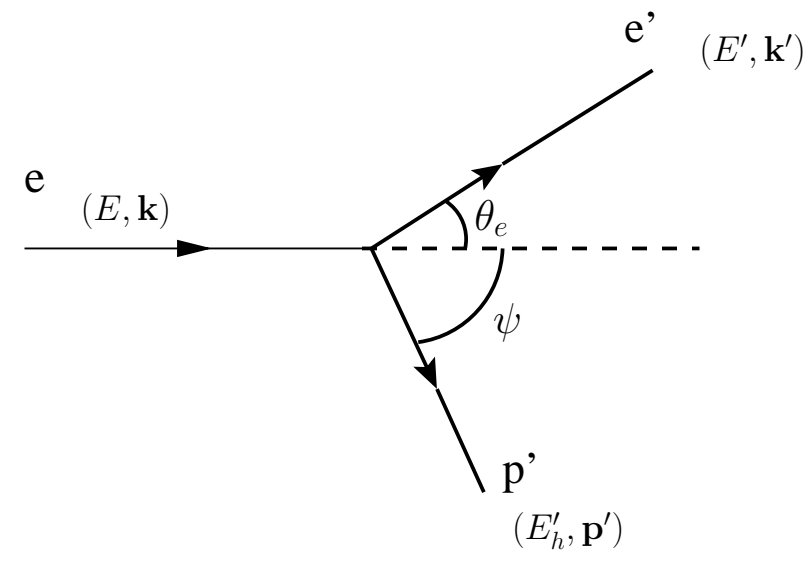

Figure 1.2: Lab frame elastic scattering.

After some manipulation of the amplitude 1.1 with the appropriate leptonic matrix element inserted (electron current) it is simple to arrive at the Rosenbluth formula. Taking the process in the lab frame and kinematic definitions consistent with Fig. 1.2 for the final state the integration over phase space variables can be performed fairly easily. The initial and final lepton 4-momentum are labeled $k$ and $k^{\prime}$ respectively, while the hadronic initial and final 4-momenta are $p$ and $p^{\prime}$. All the 4-momenta can be defined component wise for convenience and the laboratory electron scattering angle can be written in terms of these components.

$$
\begin{gathered}
k=(E, \mathbf{k}) ; \quad k^{\prime}=\left(E^{\prime}, \mathbf{k}^{\prime}\right) ; \quad p=\left(E_{h}, \mathbf{p}\right) ; \quad p^{\prime}=\left(E_{h}^{\prime}, \mathbf{p}^{\prime}\right) \\
\cos \left(\theta_{e}\right) \equiv \frac{\mathbf{k}^{\prime} \cdot \mathbf{k}}{\left|\mathbf{k}^{\prime}\right||\mathbf{k}|} \\
q^{2} \equiv\left(k-k^{\prime}\right)^{2}
\end{gathered}
$$

The definitions outlined lead directly to the Rosenbluth formula [79] for the unpo- 
larized cross section of elastic electron proton scattering in terms of the Dirac and Pauli form factors. Written in the limit where $E \gg m_{e}$ the electron mass can be neglected with extremely small error $\left(\mathcal{O}\left(\frac{m_{e}}{E}\right)\right.$ for most values of $\left.\theta_{e}\right)$.

$$
\frac{d \sigma^{e l}}{d \cos \theta_{e}}=\frac{\pi \alpha^{2}\left[\left(F_{1}^{2}-\frac{q^{2}}{4 m_{p}^{2}} F_{2}^{2}\right) \cos ^{2} \frac{\theta_{e}}{2}-\frac{q^{2}}{2 m_{p}^{2}}\left(F_{1}+F_{2}\right)^{2} \sin ^{2} \frac{\theta_{e}}{2}\right]}{2 E^{2}\left[1+\frac{2 E}{m_{p}} \sin ^{2} \frac{\theta_{e}}{2}\right] \sin ^{4} \frac{\theta_{e}}{2}}
$$

The symbol $m_{p}$ is the proton mass and the functional dependence of $F_{1}$ and $F_{2}$ has been suppressed.

It is plain to see the usefulness of such a parameterization. Simply by using the constraint that the hadronic system interacts with a typical current from QED one is able to parameterize all hadronic effects of the process into the functions $F_{1}\left(q^{2}\right)$ and $F_{2}\left(q^{2}\right)$. These functions contain all effects of the extended structure of the proton and QCD contributions to all orders of the strong coupling constant $\alpha_{s}$. Writing hadronic cross sections in this manner parameterizes QCD effects and allow one to compare to models or even direct QCD predictions.

\subsection{Inelastic Scattering off of extended particles}

Just as the Dirac and Pauli form factors parameterized the structure of the spin- $\frac{1}{2}$ hadron in the previous section, one can also parameterize an inelastic cross section from a spin- $\frac{1}{2}$ hadron with a measure of the amplitude to create arbitrary numbers of hadrons in the final state. Consider, in the one photon exchange approximation, the scattering of a lepton off of the spin- $\frac{1}{2}$ hadron.

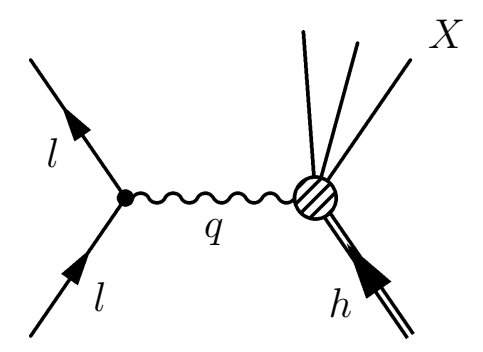

Figure 1.3: Generalized inelastic scattering. 
The symbol " $X$ " represents any hadronic final state which can be produced. Since this process includes all final hadronic states it is called inclusive scattering. The cross section can then be reported as differential in the final lepton variables. Once again, this will lead to a situation where one can parameterize all hadronic (QCD) effects into some set of scalar functions. Some simple calculations will reveal these scalar functions for the unpolarized case. The polarized case can be obtained by using the same technique, though one will find that new structure functions arise.

The matrix element for the inclusive process is in structure no different from the previous elastic case.

$$
\mathcal{M}_{\text {in }}=-\frac{e^{2} \eta_{\mu \nu}}{q^{2}}\left\langle X\left|J_{h a d}^{\mu}\right| h_{i}\right\rangle\left\langle l_{f}\left|J^{\nu}\right| l_{i}\right\rangle
$$

The leptonic current matrix element can be simply written as in the elastic case.

$$
\mathcal{M}_{\text {in }}=-\frac{e^{2} \eta_{\mu \nu}}{q^{2}}\left\langle X\left|J_{h a d}^{\mu}\right| h_{i}\right\rangle \times \bar{u}_{l}\left(k^{\prime}\right) \gamma^{\nu} u_{l}(k)
$$

At this point in the elastic calculation one introduces the functions which parameterize the QCD effects into the matrix element of the hadronic current operator as in Eqn. 1.3. The current situation does not allow such a simplification because $|X\rangle$ is not representable by a Dirac spinor asymptotically. In fact, the state $|X\rangle$ can contain any number of hadrons which need not even be fermions. Now one must proceed in a way which does not depend on what the hadronic final state is. So long as the hadronic final state is not measured one can proceed further without having an explicit form for the hadronic current.

The kinematics for inclusive scattering off of a proton by an electron can be written fairly simply in the lab frame as is represented in Fig. 1.4. The 4-momentum of the initial and final leptons are, as for the elastic case, $k$ and $k^{\prime}$. The initial proton 4-momentum is labeled $p$. When necessary (specifically throughout Appendix B) the 4-momenta of all possible particle states included in $|X\rangle$ is represented by $p_{i}$ where the index $i$ takes values from unity up to $N_{X}$ which represents the number of asymptotic particles in the final hadronic state $|X\rangle$. Only the unpolarized case will be treated in the present work so explicit spin indices are unnecessary. The symbol 


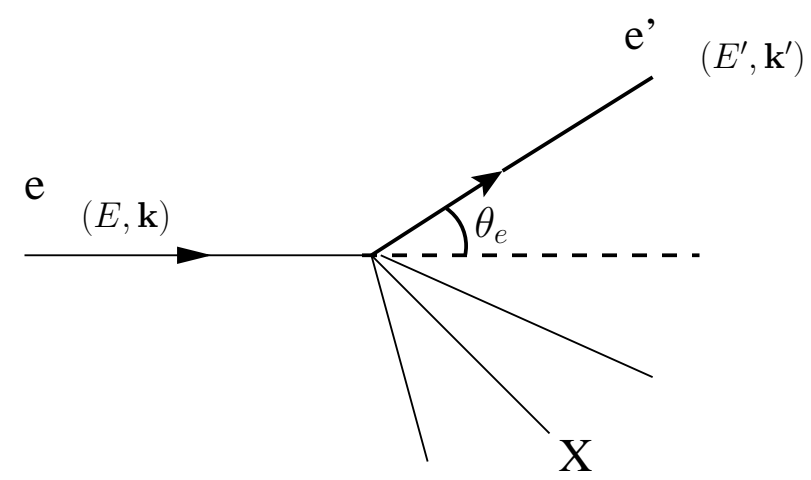

Figure 1.4: Lab frame inclusive elastic scattering.

$\sum_{\text {spins }}$ will be used to denote the sum over all of these suppressed spin indices.

$$
\begin{gathered}
k=(E, \mathbf{k}) ; \quad k^{\prime}=\left(E^{\prime}, \mathbf{k}^{\prime}\right) ; \quad p=\left(E_{p}, \mathbf{p}\right) ; \quad p_{i}=\left(E_{p_{i}}, \mathbf{p}_{i}\right) \\
\cos \left(\theta_{e}\right) \equiv \frac{\mathbf{k}^{\prime} \cdot \mathbf{k}}{\left|\mathbf{k}^{\prime}\right||\mathbf{k}|} \\
q^{2} \equiv\left(k-k^{\prime}\right)^{2}
\end{gathered}
$$

Using these kinematic variables one can write the inclusive cross section, supposing also that one specializes to a proton target for the benefit of what follows.

$$
\frac{d^{2} \sigma_{i n}}{d \Omega_{e} d E^{\prime}}=\frac{\alpha^{2}}{Q^{4}} \frac{E^{\prime}}{E} L_{\mu \nu} W^{\mu \nu}
$$

Here the symbols $L_{\mu \nu}$ and $W_{\mu \nu}$ are the leptonic and hadronic tensors described in detail in Section B.1. The definition $Q^{2} \equiv-q^{2}$ has also been made.

The previous form for the hadronic tensor does indeed provide a parameterization of QCD effects but is not a form which is simple to measure. One knows that there must necessarily still be some kinematical dependence ${ }^{7}$ in $W^{\mu \nu}$ which can be separated out since the only Lorentz structures which are available are $q^{\mu}$ and $p^{\mu}$ and the gauge constraint $q_{\mu} W^{\mu \nu}=0$ should be satisfied. Additionally, the constraint of parity invariance allows one to write a parameterization of the hadronic tensor $W^{\mu \nu}$

\footnotetext{
${ }^{7}$ By kinematical dependence it is meant that the functional form of $W^{\mu \nu}$ cannot be a completely arbitrary function of its arguments which are the only kinematical variables which exist in the problem, $q^{\mu}$ and $p^{\mu}$.
} 
with such a kinematical separation.

$$
\begin{aligned}
W^{\mu \nu}=W_{1}\left(q^{0}, Q^{2}\right) & \left(\frac{q^{\mu} q^{\nu}}{q^{2}}-\eta^{\mu \nu}\right) \\
& +W_{2}\left(q^{0}, Q^{2}\right)\left(p^{\mu}-\frac{p \cdot q}{q^{2}} q^{\mu}\right)\left(p^{\nu}-\frac{p \cdot q}{q^{2}} q^{\nu}\right)
\end{aligned}
$$

In the equation we have $q^{0}$ as a free variable since the energy transfer is no longer constrained by the 4-momentum transfer (as in elastic). Finally, the response functions $W_{1}$ and $W_{2}$ can be dropped in favor of the structure functions ${ }^{8} F_{1}\left(x, Q^{2}\right)$ and $F_{2}\left(x, Q^{2}\right)$ where $q^{0}$ has been replaced by the Bjorken variable $x=\frac{Q^{2}}{2 p \cdot q}$.

$$
\begin{aligned}
& F_{1}\left(x, Q^{2}\right)=m_{p} W_{1}\left(q^{0}, Q^{2}\right) \\
& F_{2}\left(x, Q^{2}\right)=q^{0} W_{2}\left(q^{0}, Q^{2}\right)
\end{aligned}
$$

One can then easily calculate the differential cross section for inelastic scattering where only the electron is detected (inclusive). A useful variable to use is $y=\frac{q \cdot p}{k \cdot p}$ which represents the lepton energy loss in the lab frame. The differential cross section then becomes [86].

$$
\frac{d \sigma_{i n}}{d x d Q^{2}}=\frac{4 \pi \alpha^{2}}{Q^{4}}\left[\left(\frac{1-y}{x}-\frac{m_{p}^{2} y}{s-m_{p}^{2}}\right) F_{2}\left(x, Q^{2}\right)+y^{2} F_{2}\left(x, Q^{2}\right)\right]
$$

A final popular way to write the inclusive electron scattering cross section is in terms of longitudinal and transverse cross sections which will be discussed in detail in the context of neutral meson production in Chapter 2. Defining some new variables:

$$
\begin{aligned}
W & \equiv \sqrt{(q+p)^{2}} \\
\Gamma & \equiv \frac{\alpha}{2 \pi^{2}} \frac{E^{\prime}}{E} \frac{W^{2}-m_{p}^{2}}{2 m_{p} Q^{2}} \frac{1}{1-\epsilon} \\
\epsilon & \equiv\left(1+2 \tan ^{2}\left(\frac{\theta_{e}}{2}\right) \frac{|\mathbf{q}|^{2}}{Q^{2}}\right)^{-1}
\end{aligned}
$$

\footnotetext{
${ }^{8}$ Note that while the structure functions have the same symbols as the Dirac and Pauli elastic form factors, there should be no confusion because of the context and the presence of two arguments.
} 
One can define the inclusive cross section in terms of the new variables.

$$
\frac{d \sigma_{i n}}{d W d Q^{2}}=\operatorname{det}\left[J\left(W, Q^{2}\right)\right] \Gamma\left(\sigma_{T}\left(W, Q^{2}\right)+\epsilon \sigma_{L}\left(W, Q^{2}\right)\right)
$$

The factor det $\left[J\left(W, Q^{2}\right)\right]$ is the determinant of the Jacobian which transforms from the $\left(E^{\prime}, \Omega_{e}\right)$ variable set to the invariant set.

\subsection{Resonance Production}

Although the main interest of this work is meson production, one is really interested in the physics one can probe in the resonance production channel of said meson production. The next two sections will treat the resonance production scenario (or, production of a state of definite angular momentum) without considering the final meson-plus-nucleon state. This will lay out observables that one hopes to extract from meson production data concerning production of an unstable excited state of the nucleon. In the final part of the next section, Section 1.5.3, it will be seen that one can also consider the meson production process without making any statements about the intermediate states. It is this description that will relate the most readily to experiment, but one hopes to draw relations between this description and the resonance description by assuming the resonance channel is the most important contribution. The meson production amplitudes are valuable in their own right and will make up a major part of the results of this work.

Some basic kinematic variables will be needed throughout this section. A detailed definition of all these variables can be found in Chapter 2 while a quick look up table can be found in Appendix C.

\subsubsection{Excitation of Spin- $\frac{3}{2}$ State}

The resonance region which is being examined in this work has one of the most actively studied resonant states in particle physics, the $\Delta$ state. The $\Delta$ particle comes in an isospin multiplet with four charge states having the same strangeness content $(S=0)$, these states are the $\Delta^{++}, \Delta^{+}, \Delta^{0}$ and $\Delta^{-}$. Of course the particular member of the $\Delta$ multiplet probed here is the $\Delta^{+}$, since this is the only one consistent with charge conservation of the observed $e p \rightarrow e^{\prime} p^{\prime} \pi^{0}$ reaction. 
If one restricts the theoretical analysis to the production of the $\Delta$, it is possible to parameterize the reaction with three simple functions of the momentum transfer $Q^{2}$ which are the coefficients of well defined kinematic combinations [52]. To do this one has to be able to treat an asymptotic spin- $\frac{3}{2}$ particle in a similar manner as was considered in Section 1.2. The formalism for accomplishing this goal is provided by the Rarita-Schwinger characterization of an asymptotic spin- $\frac{3}{2}$ field [78]. The basic properties of the Rarita-Schwinger field are displayed in Section A.3.

Since the process of interest here proceeds by electromagnetic excitation, one can use perturbation theory reliably. The relevant diagram for the electroproduction of an asymptotic $\Delta$ state is given in Fig. 1.5. To be precise this is the electro-

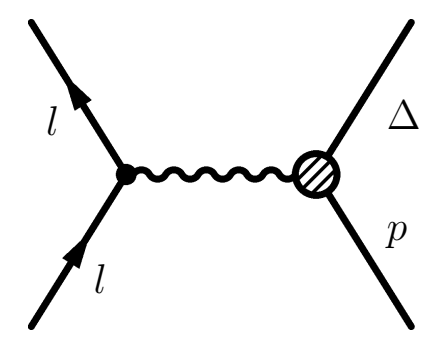

Figure 1.5: Lepton scattering excitation of an asymptotic $\Delta$.

excitation of a "particle" with $I\left(J^{P}\right)=\frac{3}{2}\left(\frac{3}{2}^{+}\right)$and third component of isospin, $I_{3}$ equal to $\frac{1}{2}$. The amplitude for this process can immediately be written in terms of a definite electromagnetic piece and a pure hadronic amplitude that will be parameterized.

$$
\mathcal{M}_{\Delta}=\frac{4 \pi \alpha \eta_{\mu \nu}}{q^{2}}\left\langle e^{\prime}\left|J^{\mu}\right| e\right\rangle\left\langle\Delta\left|J_{\text {had }}^{\nu}\right| p\right\rangle
$$

Using the Rarita-Schwinger spinor structure one can easily understand how to parameterize the vertex characterized by the hadronic piece of the above matrix element. In particular, the electromagnetic current can be written in a similar fashion as the elastic version.

$$
\left\langle\Delta\left|J_{h a d}^{\nu}\right| p\right\rangle=\bar{\psi}_{\tau} \Gamma^{\tau \nu} \psi
$$

The symbol $\Gamma^{\tau \nu}$ is a generalization of the elastic Dirac-type covariant $\Gamma^{\mu}$ used in 
elastic scattering (Eqn. 1.3). The covariant needs an extra Lorentz index to contract with the $\Delta$ spinor in the final state which now has both a Lorentz index and a spinor index (which is suppressed). The job now is to find combinations of the available momenta (here only the incoming proton momentum and the virtual photon momentum) which can be used to form a complete set of functions consistent with the necessary symmetries of $\Gamma^{\tau \nu}$. To create one such set one can simply construct Lorentz tensors (two indices) out of the available 4-vectors $q^{\mu}, p^{\mu}$ and $\gamma^{\mu}$. The important fact to remember when doing this is that $J_{\text {had }}^{\nu}$ and its matrix elements must transform as 4 -vectors which means the object $\Gamma^{\tau \nu}$ should transform with negative parity since the spinor functions $\bar{\psi}_{\tau}$ and $\psi$ each have positive parity. This means that each of the covariants should have explicit factors of $\gamma^{5}$ since any rank-2 tensor (two indices) formed out of 4 -vectors alone will have positive parity. One possible set is listed in Eqn. 1.20 and has been used by Mathews [64].

$$
\begin{aligned}
& \kappa_{1}^{\tau \nu}=\left(q^{\tau} \gamma^{\nu}-\not q \eta^{\tau \nu}\right) \gamma^{5} \\
& \kappa_{2}^{\tau \nu}=\left(q^{\tau} p^{\nu}-q \cdot \eta^{\tau \nu}\right) \gamma^{5} \\
& \kappa_{3}^{\tau \nu}=\left(q^{\tau} q^{\nu}-q^{2} \eta^{\tau \nu}\right) \gamma^{5}
\end{aligned}
$$

Note that each of these constructs also embody the all important current conservation constraint $q_{\nu} \kappa_{i}^{\tau \nu}=0$. The excitation of the delta could now be parameterized in terms of $q^{2}$-dependent coefficients of each of the above terms. These form factors would not be natural for the case at hand since we are interested in separating the electric, magnetic and Coulomb contributions as the Sachs form factors do for elastic scattering. The article of Jones and Scadron [52] and more recently the article of Pascalutsa et al. [73] (with a slightly different convention) constructs covariants which are such that their coefficients correspond to the electric, magnetic and Coulomb transition form factors $G_{E}^{*}, G_{M}^{*}$ and $G_{C}^{*}$. If one uses the 4-momentum $p_{\Delta}$ for that of the $\Delta$ particle and the definition:

$$
\Delta\left(q^{2}\right) \equiv\left(\left(m_{\Delta}+m_{p}\right)^{2}-q^{2}\right)\left(\left(m_{\Delta}-m_{p}\right)^{2}-q^{2}\right)
$$


one can write the covariants found in Eqn. 1.22.

$$
\begin{aligned}
& \kappa_{M}^{\tau \nu}=-\frac{3\left(m_{\Delta}+m_{p}\right)}{2 m_{p}\left(\left(m_{\Delta}+m_{p}\right)^{2}-q^{2}\right)} \epsilon^{\tau \nu \sigma \lambda} p_{\sigma} q_{\lambda} \\
& \kappa_{E}^{\tau \nu}=-\kappa_{M}^{\tau \nu}+6 i \Delta^{-1}\left(q^{2}\right) \epsilon^{\tau \nu \sigma \lambda} p_{\sigma} q_{\lambda} \epsilon^{\tau \nu \sigma \lambda} p_{\Delta \sigma} q_{\lambda} \gamma^{5} \frac{\left(m_{\Delta}+m_{p}\right)}{m_{p}} \\
& \kappa_{C}^{\tau \nu}=3 i \Delta^{-1}\left(q^{2}\right) q^{\tau}\left(q^{2} p^{\nu}-q \cdot p q^{\nu}\right) \gamma^{5} \frac{\left(m_{\Delta}+m_{p}\right)}{m_{p}}
\end{aligned}
$$

One can be sure that these covariant structures correspond to the correct portion of the interaction by evaluating the helicity amplitudes in the $\Delta$ rest frame with the form factors of this section and comparing to the helicity amplitudes calculated with the multipole amplitudes of Section B.2. Indeed, the assumption of a pure magnetic transition will lead to the amplitude being proportional to $\kappa_{M}^{\tau \nu}$ only. Here the Jones and Scadron conventions are followed so that one obtains the standard $G_{M}^{*}, G_{E}^{*}$ and $G_{C}^{*}$. The symbol $\epsilon^{\tau \nu \sigma \lambda}$ is the completely antisymmetric tensor with $\epsilon_{0123}=1$. The explicit factor of $\gamma^{5}$ need not appear on the magnetic covariant because its intrinsic parity has a factor of $(-1)$ relative to the electric and Coulomb excitations. Now one can write the factor $\Gamma^{\tau \nu}$ in a compact way and thus gain a parameterization of the excitation amplitude.

$$
\Gamma^{\tau \nu}=\sqrt{\frac{2}{3}}\left(G_{M}^{*}\left(Q^{2}\right) \kappa_{M}^{\tau \nu}+G_{E}^{*}\left(Q^{2}\right) \kappa_{E}^{\tau \nu}+G_{C}^{*}\left(Q^{2}\right) \kappa_{C}^{\tau \nu}\right)
$$

The factor of $\sqrt{\frac{2}{3}}$ is because we have only written the amplitude for proton excitation when the conventions for the $G^{*}$ take into account the excitation of a general nucleon.

\subsection{Multipole Amplitudes}

In Sections 1.2 and 1.3 the focus was on ways to parameterize the hadronic physics which is difficult to predict. One can also proceed in this way for cases where we have inelastic scattering and actually measure a particular outgoing hadronic state. This allows one to parameterize more specific observables and compare with the inclusive inelastic formalism of Section 1.3 since the sum of all exclusive processes must yield the inclusive process. Of particular importance is the electromagnetic 
transition to a hadronic state of particular total angular momentum. The $\Delta$ electroexcitation and subsequent decay into $\pi N$ final state studied in this work is an important example of this kind of transition. This process will give a natural means by which to describe production of pseudo-particles or "resonant" states.

\subsubsection{Electromagnetic Transition}

To bring the important aspects of an electromagnetic excitation of a target system to the foreground one can think in terms of the canonical problem of excitations of atomic states. It is often desirable to characterize various excitations by the electromagnetic multipole components which dominate their transition. For example a spin-flip transition in atomic hydrogen is dominated by a magnetic dipole amplitude $^{9}$. This means that in a multipole decomposition of incoming (or outgoing) radiation which induces a spin-flip in atomic hydrogen, the magnetic dipole or " $M 1$ " term will dominate. For the excitation of a $\Delta$ resonance via an leptonic current there are three multipoles which are not forbidden and they are the magnetic dipole (M1), electric quadrupole (E2) and Coulomb quadrupole $(C 2)$ amplitudes.

A detailed derivation of the origins of these amplitudes in terms of a formal multipole decomposition of the leptonic current matrix elements is given in Section B.2. The derivation uses irreducible tensor operators of total angular momentum $J$ with electric $(E J)$, magnetic $(M J)$ and Coulomb $(C J)$ contributions. In this derivation the amplitude for an initial state of total spin $J_{i}$ to be excited to a state with total spin $J_{f}$ is considered. These amplitudes map directly onto the $\Delta$ excitation if the final spin configuration is taken to be that of an asymptotic $\Delta$. The phases of these amplitudes are not important if the $\Delta$ is assumed to be asymptotic and the processes are measured in the total angular momentum basis. The reason for this is that in this basis each of the mentioned amplitudes have different selection rules involving the $M_{i}$ and $M_{f}$, the initial and final magnetic quantum numbers. Therefore, in this basis the amplitudes do not interfere. In this study, however, one will probe these amplitudes indirectly through a $\pi N$ decay channel where one cannot select states of definite total angular momentum and magnetic

\footnotetext{
${ }^{9}$ Though electric dipoles are in general larger than magnetic ones on the atomic scale, the electric dipole transition is forbidden in this case.
} 
quantum number $M_{f}$. These amplitudes, then, can interfere. The convention in this work is to endow these amplitudes with the phases of the $\pi N$ angular distribution amplitudes corresponding to them. The amplitudes E2, $M 1$ and $C 2$ are the matrix elements of the multipole transition operators relevant to this work. These operators are defined from the perspective of a nucleon excitation into a $\Delta$ state (not a proton or neutron individually). Isospin factors should be used to relate them back to the multipole transitions for protons or neutrons individually. In this work we will only be interested in ratios of the multipole excitation amplitudes so that even though we measure the proton excitation amplitudes, one can extract the parameters defined from the nucleon perspective.

\subsubsection{Helicity Amplitudes}

The previous sections sought to parameterize the $\Delta$ excitation in terms of multipoles or some decompositions relating to either the electric, magnetic or Coulomb character of the incoming radiation. From a covariant perspective it is much easier to parameterize the hadronic matrix elements in terms of their incoming and outgoing helicities since detailed multipole expansions are not needed for this, only the basic spin structure of each of the interacting constituents is needed. Further, it is easier to make some particular general predictions, which will be explored in Chapter 2, when only simple helicity elements are considered.

In this section the primary reaction is considered to be $\gamma^{*} p \rightarrow \Delta$. That is, a virtual photon impinging on a target proton to make a $\Delta$ particle (assumed to be an asymptotic state). The justification for considering an electroproduction reaction to be analogous to the photoproduction reactions in this way will come in Section 2.2. In fact Section 2.2 will define a "virtual photon cross section" which is differential only in the kinematic variables relating to the hadronic portion of the amplitude, though it depends on the electron invariants as well. The present section and the next section will simply assume that one can consider the relevant processes can be considered to consist of a hadronic current matrix element of the form $\left\langle f\left|J_{\text {had }}^{\mu}\right| i\right\rangle$ contracted with a 4 -vector $\epsilon_{\mu}$ which is analogous to a virtual photon polarization.

The interaction of a photon with a nucleon to produce a $\Delta$ particle can have 
two different independent helicity amplitudes. A third independent amplitude is added if one considers a virtual photon as discussed above, this corresponds to the Coulomb or longitudinal excitation. The helicity diagrams pictured in Fig. 1.6
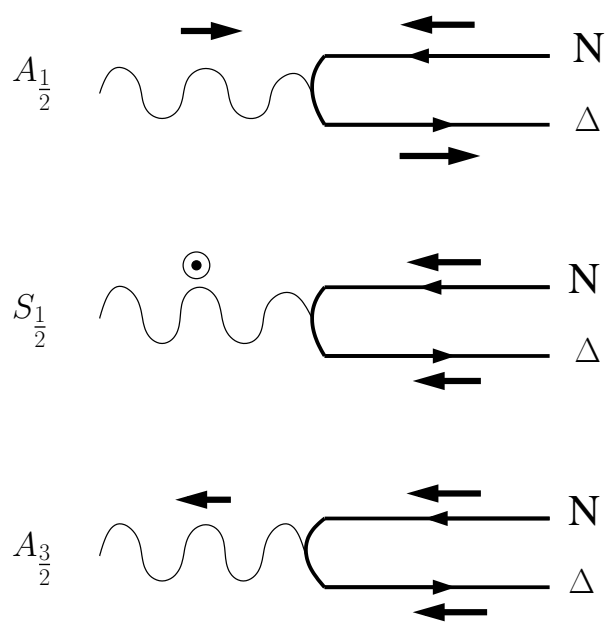

Figure 1.6: Independent helicity amplitudes for $\gamma^{*} p \rightarrow \Delta$.

represent the amplitudes for an nucleon to be excited into a $\Delta$. They can easily be defined explicitly [73].

$$
\begin{aligned}
& A_{\frac{1}{2}} \equiv-\frac{e}{\sqrt{2\left|\mathbf{q}^{* \mid}\right|}} \frac{1}{\sqrt{4 m_{N} m_{\Delta}}}\left\langle\Delta,+\frac{3}{2}\left|\mathbf{J}_{h a d} \cdot \boldsymbol{\epsilon}^{+}\right| N,+\frac{1}{2}\right\rangle \\
& S_{\frac{1}{2}} \equiv \frac{e}{\sqrt{2\left|\mathbf{q}^{*}\right|}} \frac{1}{\sqrt{4 m_{N} m_{\Delta}}}\left\langle\Delta,+\frac{1}{2}\left|J_{h a d}^{0}\right| N,+\frac{1}{2}\right\rangle \\
& A_{\frac{3}{2}} \equiv-\frac{e}{\sqrt{2 \mid \mathbf{q}^{* \mid}}} \frac{1}{\sqrt{4 m_{N} m_{\Delta}}}\left\langle\Delta,-\frac{1}{2}\left|\mathbf{J}_{h a d} \cdot \boldsymbol{\epsilon}^{-}\right| N,+\frac{1}{2}\right\rangle
\end{aligned}
$$

The amplitudes in Eqn. 1.24 are written for the nucleon (though the proton piece is probed in this experiment) and in the $\Delta$ rest frame with the quantization axis along $\mathbf{q}^{*}$, the virtual photon 3-momentum in the photon-nucleon center of mass. The current experiment probes only the proton part of the helicity matrix elements displayed here. This simply means that in all the relation equations one will find factors of the Clebsh-Gordon coefficients since the multipole amplitudes used in this work will be pertaining to the proton excitation only. The polarization vectors used in Eqn. 1.24 are those of definite states of photon helicity $\epsilon^{ \pm}=(0, \mp 1,-i, 0)$. It will 
be shown in Section 2.2 that the electron current factorizes from the hadronic part and can be written so as to separate into transverse pieces for which the 4-vectors contracted with the hadronic current matrix element are proportional to the $\epsilon^{ \pm}$.

\subsubsection{CGLN Amplitudes}

Though the previous parameterizations of the $\Delta$ excitation are useful they do not map directly onto the experimental situation. Since this work deals with data where neutral pions are produced off of proton targets, one should parameterize that interaction. In particular, resonant $\Delta$ production is not the only way to produce neutral pions (though in extracting the previously mentioned amplitudes we will assume this mode dominates at the appropriate invariant energy).

In this section we parameterize the full $\gamma^{*} p \rightarrow p^{\prime} \pi^{0}$ process in terms of multipole amplitudes in the virtual-photon and proton center of mass. This is equivalent to the $\Delta$ rest frame when a $\Delta$ is produced. Section 2.2 deals with writing the full electroproduction cross section in the center of mass frame. In that section one will see that the cross section factorizes into a "virtual photon" cross section, analogous to the neutral pion photoproduction cross section, and a kinematic factor. The current section, then, will deal only with this virtual photon cross section and will parameterize it in terms of the electric, magnetic and scalar multipoles introduced first by Chew, Goldberger, Low and Nambu [21]. The resulting CGLN multipoles are the standard used throughout this work with the already mentioned caveat that only the proton amplitudes are considered.

The virtual photon cross section ${ }^{10}$ for the process $\gamma^{*} p \rightarrow p^{\prime} \pi^{0}$ can be written in terms of a quantum mechanical amplitude in the virtual photon-proton center of mass.

$$
\frac{d \sigma^{\gamma^{*}}}{d \Omega_{\pi}^{*}}=\frac{2 W\left|\mathbf{k}_{\pi}^{*}\right|}{W^{2}-m_{p}^{2}}\left|\epsilon_{\mu} \mathcal{F}^{\mu}\right|^{2}
$$

Equation 1.25 has a pre-factor different from the $\frac{\left|\mathbf{k}_{\pi}^{*}\right|}{\left|\mathbf{q}^{*}\right|}$ introduced in the original work [21], but which reduces to this for real photons. The amplitude introduced has the factor $\epsilon_{\mu}$ as a multiplier which is suggestive of a polarization vector for an

\footnotetext{
${ }^{10}$ The concept of a virtual photon cross section will be defined in Section 2.2. At this point it is sufficient to think of this as a real photon cross section with the possibility of scalar polarization.
} 
incoming "photon." In fact, this factor can be taken to be exactly this for the case of photoproduction. For electroproduction the factor is completely analogous but with some new properties, like the possibility of corresponding to a longitudinal polarization. These properties are discussed in detail in Section 2.2. In this section we will proceed to decompose the amplitude $\epsilon_{\mu} \mathcal{F}^{\mu}$ keeping the explicit dependence on the polarization 4-vector. The amplitude can be decomposed into six independent structures [17].

$$
\epsilon_{\mu} \mathcal{F}^{\mu}=\sum_{i=1,6} F_{i}\left(W, Q^{2}, \cos \theta^{*}\right) \bar{u}\left(\mathbf{p}^{\prime *}\right) O_{i} u\left(\mathbf{p}^{*}\right)
$$

The symbols $u$ and $\bar{u}$ are the initial and final state proton spinors respectively. The complex functions $F_{i}$ parameterize the interaction and separate the contributions due to each of the $O_{i}$ operators.

$$
\begin{aligned}
& O_{1}=i \boldsymbol{\sigma} \cdot \mathbf{b} \\
& O_{2}=\boldsymbol{\sigma} \cdot \mathbf{k}_{\pi}^{*} \boldsymbol{\sigma} \cdot\left(\hat{\mathbf{q}}^{*} \times \mathbf{b}\right) \\
& O_{3}=i \boldsymbol{\sigma} \cdot \hat{\mathbf{q}}^{*} \hat{\mathbf{k}}_{\pi}^{*} \cdot \mathbf{b} \\
& O_{4}=i \boldsymbol{\sigma} \cdot \hat{\mathbf{k}}_{\pi}^{*} \hat{\mathbf{k}}_{\pi}^{*} \cdot \mathbf{b} \\
& O_{5}=-i \boldsymbol{\sigma} \cdot \hat{\mathbf{k}}_{\pi}^{*} b_{0} \\
& O_{6}=-i \boldsymbol{\sigma} \cdot \hat{\mathbf{q}}^{*} b_{0}
\end{aligned}
$$

The symbol $\boldsymbol{\sigma}$ represents the "vector" of Pauli matrices $\left\{\sigma_{x}, \sigma_{y}, \sigma_{z}\right\}$ which is used in place of the Dirac matrices because there need not be any consideration of antiprotons here. The dependence on the polarization vector is contained in the symbol $b^{\mu}$ defined in Eqn. 1.28 the spacial part of this vector is denoted by bold symbols as usual.

$$
b^{\mu}=\epsilon^{\mu}-\frac{\boldsymbol{\epsilon} \cdot \mathbf{q}^{*}}{\left|\mathbf{q}^{*}\right|} q^{\mu}
$$

The complex functions $F_{i}$ are the Lorentz invariant CGLN amplitudes. They can be expanded easily in the angular argument using the derivatives of Legendre polynomials and the CGLN multipoles $\left(E_{l \pm}, M_{l \pm}\right.$ and $\left.S_{l \pm}\right)$. The labels stand for electric, magnetic and scalar and the parameter $l$ denotes the orbital angular momentum of the final state $\pi N$ system while the label \pm denotes the final state proton spin in a 
way such that to total spin of the final state is $l \pm \frac{1}{2}$.

$$
\begin{aligned}
& F_{1}=\sum_{l}\left[P_{l+1}^{\prime}(x) E_{l+}+P_{l-1}^{\prime}(x) E_{l-}+P_{l+1}^{\prime}(x) M_{l+}+(l+1) P_{l-1}^{\prime}(x) M_{l-}\right] \\
& F_{2}=\sum_{l}\left[(l+1) P_{l}^{\prime}(x) M_{l+}+l P_{l}^{\prime}(x) M_{l-}\right] \\
& F_{3}=\sum_{l}\left[P_{l+1}^{\prime \prime}(x) E_{l+}+P_{l-1}^{\prime \prime}(x) E_{l-}-P_{l+1}^{\prime \prime}(x) M_{l+}+P_{l-1}^{\prime \prime}(x) M_{l-}\right] \\
& F_{4}=\sum_{l}\left[-P_{l}^{\prime \prime}(x) E_{l+}-P_{l}^{\prime \prime}(x) E_{l-}+P_{l}^{\prime \prime}(x) M_{l+}-P_{l}^{\prime \prime}(x) M_{l-}\right] \\
& F_{5}=\sum_{l}\left[-(l+1) P_{l}^{\prime}(x) S_{l+}+l P_{l}^{\prime}(x) S_{l-}\right] \\
& F_{6}=\sum_{l}\left[(l+1) P_{l+1}^{\prime}(x) S_{l+}-l P_{l-1}^{\prime}(x) S_{l-}\right]
\end{aligned}
$$

The variable $x$ is simply the cosine of the center of mass angle as $x \equiv \cos \theta^{*}$. The CGLN multipoles are understood to be functions of the invariant energy $W$ and momentum transfer $Q^{2}$ of the reaction. It is this dependence which is independent of the kinematic factors for the process though fully determines the measured angular distribution. The values of these multipoles will be direct consequences of the hadronic dynamics at hand and can be related easily to theoretically calculable parameters.

The relation of the CGLN multipoles to the colloquial multipoles EJ, MJ and $C J$ is important in this work. These relations can easily be obtained by parity arguments [32]. The total spin of the initial state must be conserved and obviously is equal to the total spin of the transition operator, $J$, plus or minus the spin of the initial state nucleon.

$$
J_{t o t}=\left|J \pm \frac{1}{2}\right|=\left|l \pm \frac{1}{2}\right|
$$

For electric and Coulomb multipoles one has that $|J-l|=1$ whereas for magnetic multipoles one has $J=l$. This fact means that for electric and Coulomb multipoles, $l=1$ corresponds to quadrupole $(J=2)$ or monopole $(J=0)$ amplitudes. For magnetic transitions $l=1$ is only a dipole $(J=1)$ contribution. These relations are obvious after realizing that the final state of a nucleon an pseudoscalar meson will have the parity $(-1)^{l+1}$ and that this must be equal to the initial state parity 
(strong and electromagnetic interactions conserve parity) which is $(-1)^{J}$ for electric and Coulomb multipoles but $(-1)^{J+1}$ for magnetic multipoles.

\subsection{Previous Measurements and Thrust}

In order to obtain concrete results from a study, such as in this work, one should pose an unambiguous question, gather information on said question, examine various hypothesis, perform a test (experiment) to test the ideas, and compare the final results.

The work at hand has two main thrusts. The first is to use physical observables to test if perturbative QCD (pQCD) has predictive power for production of a $\Delta$ pseudoparticle in the venue of electroproduction at large momentum transfer ${ }^{11}$. The second is to extract the relevant $\Delta$ transition parameters and pion electroproduction cross sections in a new region of kinematics, in order to compare with other previously measured kinematic regions. It is useful to have a table of the observables which are important to this study and from what perspective they are defined in this work ${ }^{12}$. As is obvious from the computations of this chapter and from Tbl.1.1,

\begin{tabular}{|c|c|c|c|}
\hline Observables & Defined For & Reaction & Comments \\
\hline$G_{M}^{*}, G_{E}^{*}, G_{C}^{*}$ & Nucleon & $e N \rightarrow e^{\prime} \Delta$ & $\Delta$ asymptotic \\
\hline$M 1, E 2, C 2$ & Nucleon & $e p \rightarrow e^{\prime} \Delta$ & $\Delta$ asymptotic \\
\hline$A_{\frac{1}{2}}, S_{\frac{1}{2}}, A_{\frac{3}{2}}$ & Nucleon & $\gamma^{*} N \rightarrow \Delta$ & $\epsilon^{ \pm, 0}$ basis \\
\hline$M_{l \pm}, E_{l \pm}, S_{l \pm}$ & Proton & $\gamma^{*} p \rightarrow p^{\prime} \pi^{0}$ & $\gamma^{*}$-p center of mass \\
\hline
\end{tabular}

Table 1.1: Reaction parameters.

the observables are not independent and are simply different ways of looking at the same physics.

There is a reason for each of the sets of parameters in Tbl. 1.1 to be introduced in this work. No sets of parameters have been used that are not needed for clarity, though there are many more sets that have been used in the past. The form factors $G_{M, E, C}^{*}$ are of interest in their own right and serve as a numerical meeting ground

\footnotetext{
${ }^{11}$ Large here means approximately $6.3-7.7 \mathrm{GeV}^{2}$.

${ }^{12}$ The reader should take care when reading other references since here the multipoles $M 1, M_{l \pm}$ etc. refer to only the proton portion of the reaction but in other references they may refer to the nucleon.
} 
for calculations of various types. Complete multipole sets (here chosen to be $E_{l \pm}$, $M_{l \pm}$ and $S_{l \pm}$ ) are used as parameters for separating angular dependence. These are natural to use when making an angular fit to a particular data set. The multipoles $E J, M J$ or $C J$ are used as a set of parameters which facilitates discussions about which incident radiation patterns will tend to excite nucleon states into $\Delta$ states and their relative strengths. These parameters essentially sweep away all the technicalities and make it easy to speak about the heart of the matter for resonant excitations. For example, if it is said that electron induced $\Delta$ excitations proceed dominantly via a magnetic dipole $M 1$ amplitude, then relative sizes of angular distributions and form factors roughly follow. Lastly, the amplitudes $A_{\frac{1}{2}}, A_{\frac{3}{2}}$ and $S_{\frac{1}{2}}$ appear in order to connect with pQCD calculations and intuitive estimations which are easier to carry out in a covariant helicity basis, especially when dealing in the limit of massless quarks and weak coupling.

It is imperative to relate the parameters, especially since calculations in pQCD prefer a covariant description and thus the helicity amplitudes are more natural. When writing these relations it is important to remember the isospin factors which occur due to the fact that some of the observables are used in this work as referring only to the proton. The helicity amplitudes are related to the colloquial multipoles as displayed in Eqn. 1.31 [41, 20]. The colloquial multipoles are taken as real.

$$
\begin{aligned}
& A_{\frac{1}{2}}=-\frac{1}{2}(M 1+3 E 2) \\
& A_{\frac{3}{2}}=\frac{\sqrt{3}}{2}(-M 1+E 2) \\
& S_{\frac{1}{2}}=-C 2
\end{aligned}
$$

An important observation regarding these relations is that the $A_{\frac{3}{2}}$ amplitude vanishes if the magnetic and electric multipoles are of equal strength. Another relation of particular importance is the connection of the so-called " $R_{E M}$ " and " $R_{S M}$ " parameters with both the colloquial multipoles and the CGLN multipoles.

$$
\begin{aligned}
R_{E M} & \equiv \frac{E 2}{M 1} \\
R_{S M} & \equiv \frac{C 2}{M 1}
\end{aligned}
$$


The ratios are then related to the pure isospin- $\frac{3}{2}$ part of the corresponding CGLN multipoles. This relationship is of the utmost importance in this kind of exclusive analysis because only the CGLN multipoles have a simple connection to the angular distributions which are experimentally observed, indeed Section 1.5.3 was devoted to previewing how the coefficients fit into an expansion in the center of mass azimuthal angle.

$$
\begin{aligned}
& \frac{E 2}{M 1}=\frac{\Im\left(\bar{E}_{1+}^{(3 / 2)}\right)}{\Im\left(\bar{M}_{1+}^{(3 / 2)}\right)} \\
& \frac{C 2}{M 1}=\frac{\Im\left(\bar{S}_{1+}^{(3 / 2)}\right)}{\Im\left(\bar{M}_{1+}^{(3 / 2)}\right)}
\end{aligned}
$$

The symbols $\bar{E}_{1+}^{(3 / 2)}, \bar{M}_{1+}^{(3 / 2)}$ and $\bar{S}_{1+}^{(3 / 2)}$ are the $\frac{3}{2}$ isospin portions of the CGLN multipoles defined for the nucleon ${ }^{13}$. What is measured in the present work is the addition of the isospin- $\frac{1}{2}$ and $\frac{3}{2}$ intermediate state contribution from proton excitations. We rationalize these two differences starting with the use of a proton target instead of the appropriate combination of neutron and proton targets (nucleon). Since the transition operators in question also have isospin structure it is useful to examine the transitions in terms of the natural isospin states. This means that the nucleon and $\Delta$ multiplets should rightfully be considered as different aspects of the same particle. The isospin structure of the transition operators may not be trivial in the case of photo or electroproduction of definite isospin states since the electromagnetic interaction does not conserve isospin. Assuming that there is no isotensor transition operator, the ratios of the multipole operators will be equivalent from excitation of either an initial nucleon or proton given that only the isospin- $\frac{3}{2}$ intermediate state component is used [69]. Though there seemed to be experimental evidence of an isotensor transition [80], the assumption that there is no isotensor transition operator seems to be a good one based on many more subsequent measurements $[13,42,90,26,23,22]$.

It is well known that a final state with two hadrons of non-zero isospin is in

\footnotetext{
${ }^{13}$ The notation used in this work is such that a bar on a CGLN multipole will specify that the multipole is from the nucleon perspective, otherwise it is assumed to be defined from the proton perspective.
} 
general not in a state of definite total isospin. Therefore it is impossible to "pick" out the $\frac{3}{2}$ isospin component (corresponding to the $\Delta$ ) as Eqn. 1.33 would necessitate. Isolating this contribution would require either a more complex fitting procedure than will be pursued in this work [31, 56], or a simplifying assumption. In this case the simplifying assumption will be that the $\Delta$ resonance dominates at the pole position $^{14}$. The amplitudes will then be dominated by the $\frac{3}{2}$ contribution and be purely imaginary. In the case of the electromagnetic ratio one has the following when evaluated at the resonance pole $W=1.232 \mathrm{GeV}$.

$$
\begin{aligned}
\frac{\Re\left(E_{1+}^{*} M_{1+}\right)}{\left|M_{1+}\right|^{2}} & =\frac{\Re\left(E_{1+}\right) \Re\left(M_{1+}\right)+\Im\left(E_{1+}\right) \Im\left(M_{1+}\right)}{\Re\left(M_{1+}\right)^{2}+\Im\left(M_{1+}\right)^{2}} \\
& \simeq \frac{\Im\left(E_{1+}\right)}{\Im\left(M_{1+}\right)} \\
& \simeq \frac{\Im\left(E_{1+}^{(3 / 2)}\right)}{\Im\left(M_{1+}^{(3 / 2)}\right)}
\end{aligned}
$$

A similar relation holds for a similar combination involving the $S_{1+}$ interference term. All together one can see that:

$$
\begin{aligned}
& R_{E M} \simeq \frac{\Re\left(E_{1+}^{*} M_{1+}\right)}{\left|M_{1+}\right|^{2}} \\
& R_{S M} \simeq \frac{\Re\left(S_{1+}^{*} M_{1+}\right)}{\left|M_{1+}\right|^{2}}
\end{aligned}
$$

These are the parameters that can be easily extracted from the data in the current analysis with some assumptions. Of course, if enough data is collected with different reactions including reactions which measure polarization observables, the CGLN multipoles can be extracted individually. This sort of fit is beyond the scope of this work but will be performed when the data analyzed here is combined with the worlds data for similar reactions. The assumption that the $\Delta$ contribution dominates will be seen to be a strong one which becomes less valid as $Q^{2}$ increases.

Finally, the form factors can be related to the CGLN multipoles [17] which

\footnotetext{
${ }^{14}$ All relations from this section implicitly assume evaluation at the resonance pole position for the $\Delta$ which is taken to be $W=1.232 \mathrm{GeV}$.
} 
are accessible from experiment.

$$
\begin{aligned}
& \Im\left(M_{1+}^{(3 / 2)}\right)=\eta_{b} \sqrt{\frac{2}{3}} G_{M}^{*} \\
& \Im\left(E_{1+}^{(3 / 2)}\right)=-\eta_{b} \sqrt{\frac{2}{3}} G_{E}^{*} \\
& \Im\left(S_{1+}^{(3 / 2)}\right)=-\eta_{b} \frac{\left|\mathbf{q}^{*}\right|}{2 m_{\Delta}} \sqrt{\frac{2}{3}} G_{C}^{*}
\end{aligned}
$$

As usual $\mathbf{q}$ is the 3-momentum transfer to the initial nucleon (in this case proton) system. The symbol $\eta_{b}$ is a kinematic pre-factor. The $G^{*}$ are obviously devoid of this shape since they come from an analysis which considers the $\Delta$ to be asymptotic. The factors of $\sqrt{\frac{2}{3}}$ are isospin factors considering that the measured CGLN multipoles correspond only to the proton in this work.

$$
\begin{aligned}
\eta_{b} & \equiv N \sqrt{\frac{3 m_{p}\left|\mathbf{q}^{*}\right|}{8 \pi m_{\Delta}\left|\mathbf{k}_{\pi}^{*}\right| \Gamma}} \\
N & \equiv \frac{e}{2 m_{p}} \sqrt{\frac{m_{\Delta}\left|\mathbf{q}^{*}\right|}{m_{p}}} \frac{1}{\left[1+\frac{Q^{2}}{\left(m_{p}+m_{\Delta}\right)^{2}}\right]^{\frac{1}{2}}}
\end{aligned}
$$

It is obvious that the $\eta_{b}$ factor endows the CGLN multipoles with the with the correct resonance properties showing up as kinematic factors. As is usual, $e$ is the electric charge of the incoming and outgoing nucleon (proton) momenta. The symbol $W$ is the invariant energy of the reaction and $\Gamma$ is the full width at half maximum (decay width).

In this work we are using the prescription of "resonance dominance" quite loosely. Generally, it is not good practice to assume all enhancements in unpolarized cross section spectra are due to quasi-bound states of the Hamiltonian (composite particles). One must see that both the size and phase of an amplitude change as expected through a given energy region to have reasonable evidence that there is a quasi-bound state at a particular energy. In other language, one could say that there is a "pole" in the S-matrix at this energy. In this work one can relax the strict need for extracting size and phase of amplitudes since the $\Delta$ is a well established and reasonably well separated state in the hadronic spectrum. The initial observation 
was by Fermi and collaborators over 50 years ago [4]. Since that time there have been other works confirming the resonant structure of the $\Delta$ excitation and indeed that at low energy the magnetic dipole transition seems to nearly maximal from the prespective of unitarity [14].

As a final note it should be mentioned that sometimes in the literature the scalar multipoles $S_{l \pm}$ are replaced by the longitudinal multipoles with $S_{l \pm}=\frac{|\mathbf{q}|}{q^{0}} L_{l \pm}$.

\subsection{1 pQCD Expectations}

One of the most interesting aspects of hadronic physics is the possible onset of pQCD at high momentum transfers. In this framework quark and gluon degrees of freedom should describe hadronic observables via a well defined perturbative expansion. It is predicted that at very large momentum transfer a well behaved perturbation series can be used for QCD calculations [60]. Perturbative QCD calculations have varying degrees of success for hadronic observables, thus it becomes important to quantify which observables can be described by pQCD and at what momentum scale these descriptions become valid.

The current data has two important indicators relating to the validity of pQCD. The first is related to helicity conservation. A massless vector particle (like a gluon or photon) cannot transfer helicity to a massless fermion (like a quark). Given this fact, the helicity of the initial and final state hadrons cannot be changed in the limit that the quarks are massless. Since the high energy limit of QCD is chirally symmetric, this situation should be attained. It turns out that in the rest frame of a produced $\Delta$ state in the reaction $e p \rightarrow \Delta \rightarrow e^{\prime} p^{\prime} \pi^{0}$ the $E 2$ amplitude will exactly equal the $M 1$ amplitude if helicity is conserved ${ }^{15}$. This means that the $\frac{E 2}{M 1}$ ratio should approach unity in this limit. Equation 1.31 shows that this will be the situation if the helicity conserving amplitude, $A_{\frac{1}{2}}$, is non-zero while the helicity non-conserving amplitude, $A_{\frac{3}{2}}$, is zero. It is clear from previous studies that this is far from realized in data up to a momentum transfer of $Q^{2} \sim 4.0 \mathrm{GeV}$, in fact the value is small in magnitude and negative [40]. The second important indicator for relevance of $\mathrm{pQCD}$ is a $\frac{1}{Q^{3}}$ behavior of the helicity conserving amplitude in the

\footnotetext{
${ }^{15}$ Assuming pure resonance excitation the amplitudes are both purely imaginary at the resonance position.
} 
$\gamma^{*} p \rightarrow \Delta$ reaction [20]. This will reveal itself as a fall off for the transition form factor $G_{M}^{*}$ consistent with the dipole form $\left(Q^{-4}\right.$ at high $\left.Q^{2}\right)$. A fall off of the form factor which is faster than this will indicate that soft processes are still important.

\subsubsection{Previous World Data}

Previous measurements of the important parameters for $\Delta$ production unambiguously show that one has not reached a kinematic region where pQCD contributions become dominant up to a momentum transfer of approximately $Q^{2}=5.0$ $\mathrm{GeV}^{2}$. However, one can also ask if the data is beginning to show an "expected" interpolating behavior between the values at the currently accessible kinematic regions and the pQCD predictions. This "expected" behavior depends, of course, on estimation and intuition since the true behavior above the previously mentioned momentum transfer is not known. A some simple expectations have been put fourth based on the knowledge of the behaviors of other known form factors and specific pQCD predictions [20]. Using these expectations as a bench mark, it would be very significant indeed $^{16}$ if the ratio $R_{E M}$ had a sign change in the range $2.5 \leq Q^{2} \leq 10.0$ $\mathrm{GeV}^{2}$.

Figure 1.7 displays the current situation for the ratio $R_{E M}$. The real photon point at $Q^{2}=0$ began small in magnitude and negative in sign. This situation has had no drastic change up to $Q^{2}$ of about $5.0 \mathrm{GeV}^{2}$. The current experimental situation for the scalar to magnetic ratio, $R_{S M}$ is displayed in Fig. 1.8. In Figs. 1.7 and 1.8 the data are separated by the collaboration from which they were obtained. The MAMI (Mainzer Microtron) collaboration has been able to obtain much precision data on the subject $[77,3,35,85,84]$. The Jefferson Laboratory CLAS (Cebaf Large Acceptance Spectrometer) collaboration has also obtained a large amount of data displayed in the previous figures from a variety of publications $[55,9,87]$. The Jefferson Laboratory spectrometer Halls A [57] and C [40] have also made significant contributions to the question of the structure of the $N \rightarrow \Delta$ transition. Several other collaborations have also made important contributions to the measurement of the multipole ratios. These collaborations are the electron accelerator ELSA (Uni-

\footnotetext{
${ }^{16}$ Very significant, but not quite conclusive.
} 


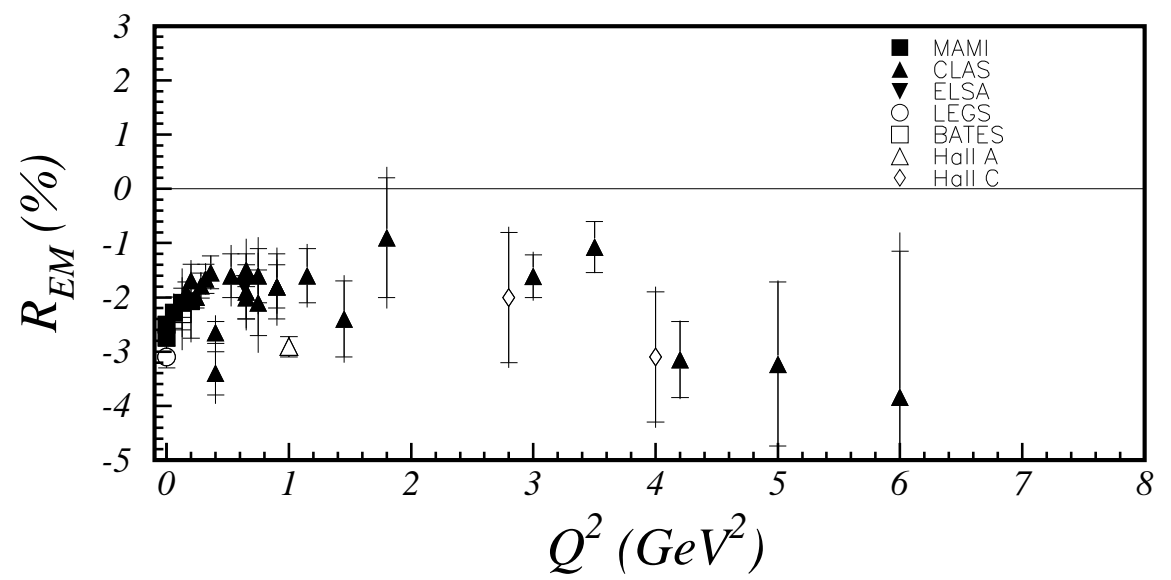

Figure 1.7: Current experimental situation for $R_{E M}$. The points are separated by laboratory or detector. The outer error bars are the sum (in quadrature) of the statistical and systematic errors.

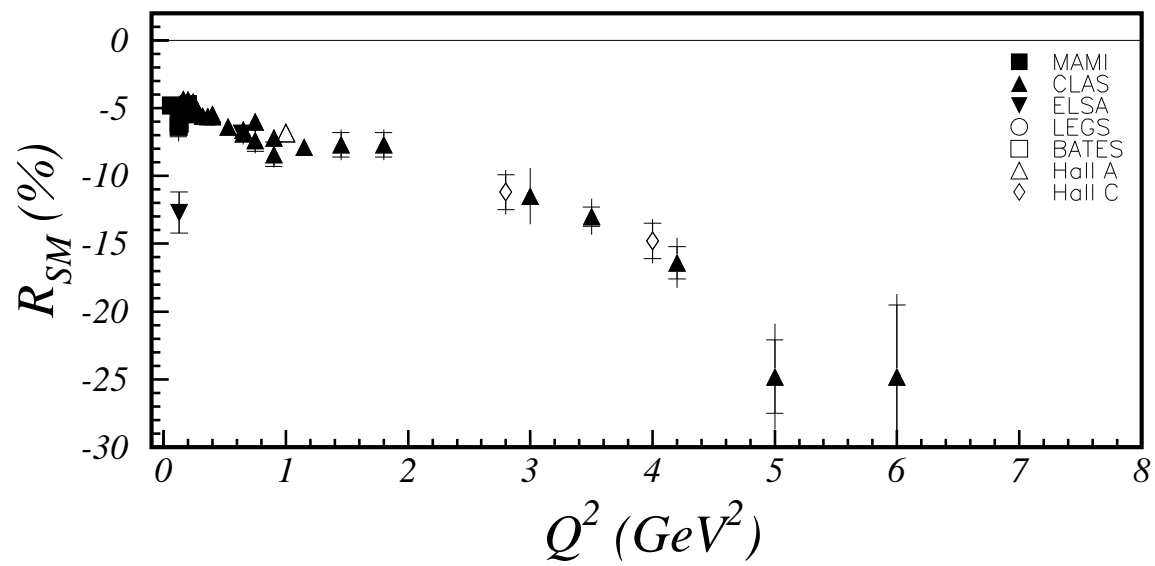

Figure 1.8: Current experimental situation for $R_{S M}$. The points are separated by laboratory or detector. The outer error bars are the sum (in quadrature) of the statistical and systematic errors.

versity of Bonn) collaboration [10], the LEGS (Brookhaven) collaboration [82, 15] and the BATES (MIT) collaboration [83].

The transition form factor $G_{M}^{*}$ is another traditional parameter that has been measured concerning the $\Delta$ excitation. Since the multipole $M_{1+}$ has long since dominated all relevant multipoles for neutral pion production in the vicinity of the $\Delta$ resonance pole, the best data in this region of invariant energy exists for $G_{M}^{*}$. Therefore we will concern ourselves only with this transition form factor in this work 
even though the situation of $M_{1+}$ dominance is fast-changing and it is not likely that one will be able to straightforwardly extract the transition form factor $G_{M}^{*}$ much above $Q^{2}$ of $5.0 \mathrm{GeV}^{2}$ by ignoring contributions in the cross section of multipoles like $E_{0+}$. Figure 1.9 shows that the magnetic transition form factor is falling much faster than the dipole form factor, $G_{D}$.

$$
G_{D}=\frac{1}{\left(1+\frac{Q^{2}}{0.71(\mathrm{GeV})}\right)^{2}}
$$

Asymptotically, this form factor should have the same large $Q^{2}$ dependence as the dipole form factor and therefore this is an indicator that soft QCD processes are still very important for the calculation of the electromagnetic nucleon to $\Delta$ transition.

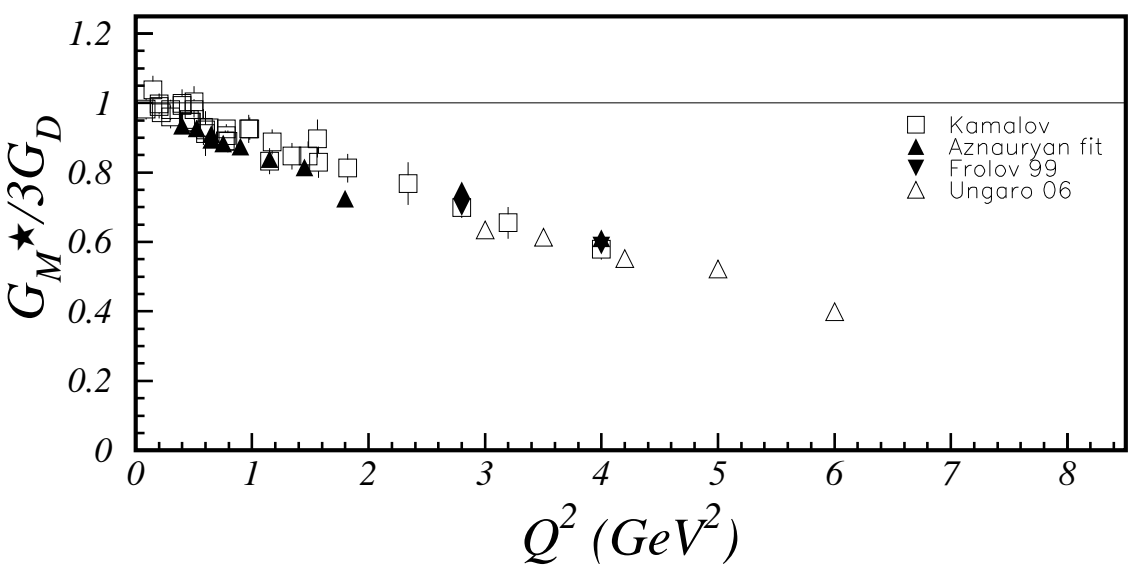

Figure 1.9: Current experimental situation for $G_{M}^{*}$ in the Jones and Scadron convention [52]. The points are separated by primary investigator.

This work will improve the current experimental situation. First and foremost it will provide more neutral pion production cross section data in a range of invariant energies from just above threshold to about $W=1.4 \mathrm{GeV}$. This data will provide new points for phenomenological fitting and allow the extraction of not only resonant properties of the delta but a stronger constraint on the various neutral pion production background contributions. It will also be clear from this experimental investigation how one must proceed to measure some of these hadronic properties at larger momentum transfer. It is very likely that the approach to the next generation 
experiments will need to be significantly different for studies of the $\Delta$ due to the rapid decrease of its contribution to the total cross section. 


\section{CHAPTER 2 \\ Kinematics}

\subsection{Kinematics Before Dynamics}

It is usually the case in physics that one is interested in the dynamics of a particular phenomenon because it is this aspect which can be stripped of any specific application and applied very broadly. That is not to say, however, that the kinematics of a process are necessarily trivial. Particle interactions which have more than two particles in the final state are a good example of complicated kinematics [70]. The kinematical complication is an important reason why Monte Carlo codes are used so frequently in modeling particle interactions with particular detector systems. Despite these complications most would advocate the position that kinematics should be separated from dynamical considerations to the largest extent possible. Indeed, the parameterization of hadronic effects in terms of form factors and structure functions as was seen in Sections 1.2 and 1.3 is in just that spirit. The present chapter will seek to do so for the most important processes related to the pseudoscalar meson production cross section extraction.

\subsection{Production of $\pi^{0}$ Mesons}

Considering the production of a single neutral pion $\left(\pi^{0}\right)$ requires one to have a detailed understanding of the interaction kinematics which depends at least in part on reaction mechanisms (though we strive to minimize this dependence ${ }^{1}$ ). The single dynamical assumption which is made that makes kinematics simpler and indeed even allows straightforward parameterization of the dynamics is the single photon perturbative approximation. The results of this work relating to dynamical form factors are valid only to the extent that this approximation is satisfied. It is also very important to understand the process at hand in both the laboratory and

\footnotetext{
${ }^{1}$ One will see that the kinematics depend on the reaction mechanisms in mostly a logistical way. If more than one photon is exchanged between the lepton and hadron current it will make the definition of a hadron-virtual photon center of mass frame difficult. The hadron-virtual photon center of mass frame will be used extensively in what follows.
} 
the center of mass frames, to be defined in what follows. This is essential because the measuring apparatus are understood more fully in the lab frame while the dynamical predictions are simplified in the center of mass frame.

It is natural to examine the differential cross section for a neutral pion $\left(J^{P}=\right.$ $0^{-}$) from the following exclusive reaction.

$$
e+p \rightarrow e^{\prime}+p^{\prime}+\pi^{0}
$$

Including only leading nontrivial order in the fine structure constant $\alpha$ one can go a considerable distance in the calculation of the cross section just by the knowledge of the interaction with a pure QED system. The kinematics for such a process are displayed in Fig.2.1. In electroproduction of a single meson five kinematic variables

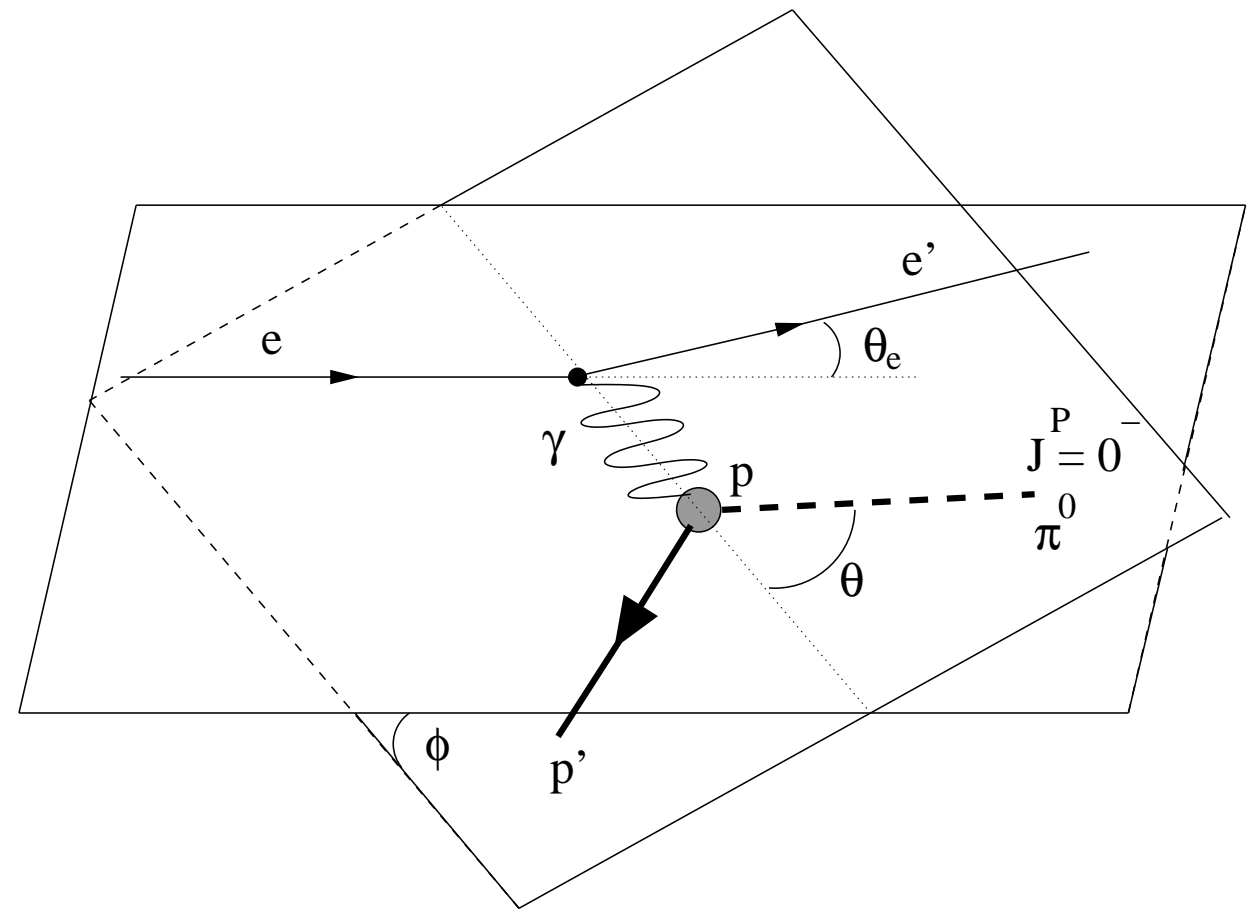

Figure 2.1: Lab frame neutral pion production.

are needed to specify the unpolarized reaction fully. Assuming that the energy of the incident electron is known these variables can be chosen to be $E^{\prime}$, the electron angles $\Omega_{e}$ and the meson angles $\Omega_{\pi}$. Using the fact that the incident energy $E$ is known and the target energy is simply $m_{p}$, one can completely specify the interaction. Given 
this convention, the 5-fold differential cross section can be obtained as a function of the mentioned variables. The definitions of the solid angles as they correspond to Fig. 2.1 should be noted.

$$
\begin{aligned}
& d \Omega_{e} \equiv \sin \theta_{e} d \theta_{e} d \phi_{e} \\
& d \Omega_{\pi} \equiv \sin \theta d \theta d \phi
\end{aligned}
$$

It must be mentioned that the variable $\phi_{e}$ is completely ignorable physically in the context of the definitions of Fig. 2.1 with unpolarized scattering. The variable simply specifies the angle of the reaction plane with respect to some external reference. The differential cross section may not depend on this variable since a rotation of this coordinate corresponds to the same physical situation. It will, however be important to understand that the differential cross section will be distributed uniformly in this variable. Neglecting this fact will lead to logistical problems like invalid normalization for a possible Monte Carlo model of the process. The cross section then takes the following general form where the order of the derivative has been suppressed but is obvious from the dependency.

$$
\frac{d \sigma}{d \Omega_{e} d E^{\prime} d \Omega_{\pi}}=f\left(\theta_{e}, E^{\prime}, \Omega_{\pi}\right)
$$

It is useful to express as many of the variables as possible through the use of Lorentz invariants. In so doing we de-emphasize the dependence of the cross section on ignorable coordinates such as $\phi_{e}$ and emphasize the fundamental energy and momentum variables which influence the interaction in all reference frames. This procedure also makes one able to predict some simple dynamical effects from the covariant procedures for calculating QED matrix elements (the Feynman rules). Another happy side effect is the fact that the lepton current portion will completely factorize in a frame-invariant way, which will help one to write the amplitudes in terms of only hadronic variables multiplied with some known (and frame invariant) QED factors. The most obvious new coordinate which is suggested from the lab frame kinematics and the treatment of the elastic process in Section 1.2 is the momentum transfer from the electron to the proton. In view of the one-photon exchange approximation 
this can be viewed as the 4-momentum of the exchanged photon ${ }^{2}$. This understanding of the 4-momentum transfer will be especially useful when moving to the center of mass frame.

$$
q^{\mu}=k^{\mu}-k^{\prime \mu}
$$

As before $k$ is the 4-momentum of the incoming electron and $k^{\prime}$ is the 4-momentum of the outgoing electron. If one is to define the incoming proton 4-momentum to be $p$ and the outgoing to be $p^{\prime}$, the two electron invariants become apparent.

$$
\begin{aligned}
& Q^{2} \equiv-q^{2}=2 E E^{\prime}\left(1-\cos \theta_{e}\right) \\
& W \equiv \sqrt{(q+p)^{2}}=\sqrt{m_{p}^{2}+2 q^{0} m_{p}-Q^{2}}
\end{aligned}
$$

The rightmost equalities in Eqn. 2.5 hold in the lab frame. This definition makes it possible to write the previous differential cross section partially in terms of invariants.

$$
\frac{d \sigma}{d Q^{2} d W d \Omega_{\pi}}=g\left(Q^{2}, W, \Omega_{\pi}\right)
$$

The dependence on the leptonic variables is now completely in terms of invariants which can be calculated in any frame. It will be seen that $g$ is a very special distribution with respect to its arguments, in particular, it factorizes keeping the electron piece separate. To be complete all the variables should be unambiguously defined. In addition to the previously defined 4-vectors one has the outgoing pion 4-momentum $k_{\pi}$. The non-covariant vector components can easily be defined and their center of mass frame (which has yet to be defined) counterparts are denoted as per the convention in Appendix C.

$$
\begin{aligned}
& k=(E, \mathbf{k}) ; \quad k^{\prime}=\left(E^{\prime}, \mathbf{k}^{\prime}\right) ; \quad p=\left(E_{p}, \mathbf{p}\right) ; \quad p^{\prime}=\left(E_{p}^{\prime}, \mathbf{p}^{\prime}\right) ; \quad k_{\pi}=\left(E_{\pi}, \mathbf{k}_{\pi}\right) \\
& \cos \left(\theta_{e}\right) \equiv \frac{\mathbf{k}^{\prime} \cdot \mathbf{k}}{\left|\mathbf{k}^{\prime}\right||\mathbf{k}|} \\
& \cos (\theta) \equiv \frac{\mathbf{q} \cdot \mathbf{k}_{\pi}}{|\mathbf{q}|\left|\mathbf{k}_{\pi}\right|}
\end{aligned}
$$

The $\phi$ coordinate takes a bit more thinking to define without reference to compo-

\footnotetext{
${ }^{2}$ With the caveat that the internal (unobservable) photons need not be on shell.
} 
nents. Using an inverse cosine will lead to an ambiguity since $\phi$ must range from $-\pi$ to $\pi$. A simple remedy can be used utilizing the $\operatorname{sgn}(\cdot)$ function. The notation $\hat{\mathbf{u}}$ will also be used to denote the unit vector in the $\mathbf{u}$ direction.

$$
\phi=\operatorname{sgn}\left(\widehat{\mathbf{k} \times \mathbf{k}^{\prime}} \cdot \hat{\mathbf{k}}_{\pi}\right) \arccos \left(\widehat{\mathbf{k} \times \mathbf{k}^{\prime}} \cdot \widehat{\mathbf{p}^{\prime} \times \mathbf{k}_{\pi}}\right)
$$

It has been mentioned that it is desirable to move to the hadron-virtual photon center of mass frame. Kinematically it is easy to see why this is desirable, it essentially replaces three body final state with the two body version. Dynamical considerations for the pure QED portion of the matrix element must, however, be taken into account. Though it was suggested that the lepton current portion of the matrix element will factorize, this has not been shown. In particular, one would like to treat the hadronic cross section as the interaction of a virtual photon with a target hadron and forget the leptonic current completely aside from some pre-factor to the amplitude which is a function of the Lorentz invariants $Q^{2}$ and $W$. Even though the details of this are not clear yet, the picture of the kinematic variables in the hadron-virtual photon center of mass is clear. Lorentz boosting along the direction of the momentum transfer will do the job.

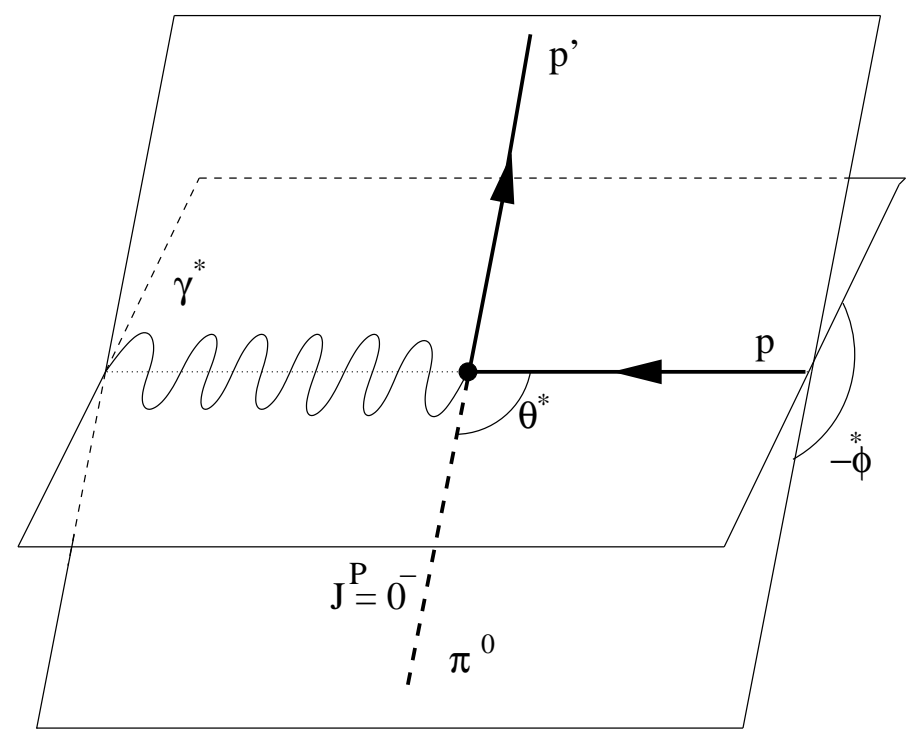

Figure 2.2: Center of mass frame neutral pion production. 
The matrix element for pion electroproduction can be written down unambiguously. The pion production matrix element in the one photon exchange approximation will be as follows.

$$
\mathcal{M}_{\pi}=\frac{4 \pi \alpha \eta_{\mu \nu}}{q^{2}}\left\langle e^{\prime}\left|J^{\mu}\right| e\right\rangle\left\langle\pi^{0}, p^{\prime}\left|J_{h a d}^{\nu}\right| p\right\rangle
$$

Equation 2.10 looks remarkably similar to the photoproduction matrix element which can be written out just as simply.

$$
\mathcal{M}_{\pi}^{\gamma}=e \tilde{\epsilon}_{\mu}\left\langle\pi^{0}, p^{\prime}\left|J_{h a d}^{\mu}\right| p\right\rangle
$$

The $\tilde{\epsilon}_{\mu}$ is the photon polarization vector for the incoming real photon. It is easy to see that the electroproduction and photoproduction amplitudes are so similar in structure that a redefinition of the "photon polarization" will suffice to have them take exactly the same form. It turns out that one can actually go considerably further with this analogy and be able to talk about "polarizations" in much the same way as for photoproduction ${ }^{3}[62]$. One must recall that the photoproduction amplitude satisfies the Ward identity (gauge invariant) so that if $\tilde{q}$ is the incoming photon 4-momentum then one has the following with $T^{\mu} \equiv e\left\langle\pi^{0}, p\left|J_{\text {had }}^{\mu}\right| p\right\rangle$.

$$
\tilde{q}_{\mu} T^{\mu}=0
$$

There are similar relations for the electroproduction amplitude due to current conservation. Given the following definition of $\epsilon_{\mu}$ these relations follow.

$$
\begin{aligned}
\epsilon^{\mu} & \equiv\left\langle e^{\prime}\left|J^{\mu}\right| e\right\rangle \\
q_{\mu} T^{\mu} & =0 \\
q_{\mu} \epsilon^{\mu} & =0
\end{aligned}
$$

The amplitude for the pion production can be made into a cross section through squaring and kinematic factors. Leaving the kinematic factors aside one

\footnotetext{
${ }^{3}$ Of course, photoproduction does not have a longitudinal polarization.
} 
can compare the unpolarized pion electroproduction to photoproduction.

$$
\begin{aligned}
\sum_{\text {spins }}\left|\mathcal{M}_{\pi}\right|^{2} & =\left(\frac{e}{q^{2}}\right)^{2} \sum_{\text {lspins }} \epsilon_{\mu}^{*} \epsilon_{\nu} \sum_{\text {hspins }} T^{\mu *} T^{\nu} \\
\sum_{\text {spins }}\left|\mathcal{M}_{\pi}^{\gamma}\right|^{2} & =\tilde{\epsilon}_{\mu}^{*} \tilde{\epsilon}_{\nu} \sum_{\text {hspins }} T^{\mu *} T^{\nu}
\end{aligned}
$$

The portion of the photon matrix element squared which relates to the incoming photon polarizations is explicitly written in Eqn. 2.14. This portion of the expression can be shown to be derivable from a bona-fide density operator for the incoming photon states $\tilde{\rho}[28]^{4}$. Using the states $\left|\gamma^{i}\right\rangle$ as pure states for the photon ${ }^{5}$ spin portions of the incoming photon states one can explicitly write it. Here the index $i$ takes values in $\{1,2,3\}$. The indices $\{1,2\}$ will represent two independent transverse pure states and the index $\{3\}$ will be a longitudinal pure state. Real photons of course cannot have longitudinal polarization but we keep this state for the purpose of easy generalization to electroproduced "virtual" photon density matrix. For a real photon state $|k, \lambda\rangle$ (where $\lambda$ is the helicity) we simply agree that $\left\langle 0\left|A^{\mu}(x)\right| k, 0\right\rangle=0$. A remark should be made about real photons in this venue. It would be logical to think that a 4 -vector field such as the photon field $A^{\mu}$ would have 4 degrees of freedom. This is not so in the case of massless photons as above the claim is that there are only two. The longitudinal polarization can be considered the mode which is removed by the physical constraint ${ }^{6} \partial_{\mu} A^{\mu}(x)^{+}|\psi\rangle=0$. This constraint has no analog for the lepton or "virtual" photon case. The other mode that is "removed" while quantizing the photon field corresponds to a pure gauge mode and comes from considering the vacuum as being free of any modes, when in general it can have any number of pure gauge modes and still represent the same physical state [63].

First we make an explicit calculation for an incident photon, then we generalize the density matrix ideas for use with lepton currents. A basis for the $\epsilon^{\mu}$ is necessary and will contain three basis vectors, including a longitudinal polarization but neglecting a possible pure gauge polarization. The quantization axis is chosen

\footnotetext{
${ }^{4}$ Beware of the metric convention in this reference, it has cost at least one bright physicist half a day's worth of algebra.

${ }^{5}$ For a $3 \times 3$ density matrix one needs at most 3 pure states.

${ }^{6}$ In a covariant canonical photon quantization scheme.
} 
along the direction of the photon momentum.

$$
\begin{aligned}
\epsilon_{x}^{\mu} & \equiv(0,1,0,0) \\
\epsilon_{y}^{\mu} & \equiv(0,0,1,0) \\
\epsilon_{L}^{\mu} & \equiv\left(\tilde{q}^{0}, 0,0, \tilde{q}^{3}\right) / \sqrt{\tilde{q}^{2}}
\end{aligned}
$$

Note that specifying the spacial portion of any of the basis vectors in Eqn. 2.15 completely specifies the vector as long as one agrees that the only way one can have a component along the $\tilde{\mathbf{q}}$ direction is by addition of an $\epsilon_{L}^{\mu}$ component. Now one can give a typical specification of the density operator $\tilde{\rho}$ and relate this to the photon polarization portion of $\mathcal{M}_{\pi}^{\gamma}$.

$$
\tilde{\rho} \equiv a\left|\gamma^{1}\right\rangle\left\langle\gamma^{1}|+b| \gamma^{2}\right\rangle\left\langle\gamma^{2}|+c| \gamma^{3}\right\rangle\left\langle\gamma^{3}\right|
$$

with,

$$
|a|^{2}+|b|^{2}+|c|^{2}=1
$$

This means that the density matrix can be more general than simply a projector for some pure state. This can be simply related to the factor in $\mathcal{M}_{\pi}^{\gamma}$.

$$
\tilde{\epsilon}_{\mu}^{*} \tilde{\epsilon}_{\nu}=\left\langle 0\left|A_{\mu}(x) \tilde{\rho} A_{\nu}(x)\right| 0\right\rangle
$$

It has been noted that the spacial components of this object will define the behavior of all components, so it is useful for one to examine the following matrix.

$$
\tilde{\rho}_{i j} \equiv \tilde{\epsilon}_{i}^{*} \tilde{\epsilon}_{j}
$$

Now use the pure states $\left|\gamma_{x}^{\perp}\right\rangle$ (linear polarized in $\mathrm{x}$ ), $\left|\gamma_{R}\right\rangle$ (right circular polarized) and $\left|\gamma_{0}\right\rangle$ (longitudinal polarized) to define the states $\left|\gamma^{1}\right\rangle,\left|\gamma^{2}\right\rangle$ and $\left|\gamma^{3}\right\rangle$ of the density operator. If $a=1$ then the density matrix obtained is:

$$
\tilde{\boldsymbol{\rho}}=\left(\begin{array}{lll}
1 & 0 & 0 \\
0 & 0 & 0 \\
0 & 0 & 0
\end{array}\right)
$$


On the other hand if we use the above state specification and with $b=1$ one can find the density matrix for a circularly polarized incoming photon.

$$
\tilde{\boldsymbol{\rho}}=\left(\begin{array}{ccc}
\frac{1}{2} & \frac{-i}{2} & 0 \\
\frac{i}{2} & \frac{1}{2} & 0 \\
0 & 0 & 0
\end{array}\right)
$$

Finally, if one sets $c=1$ for a pure longitudinal polarization the density matrix becomes $^{7}$ :

$$
\tilde{\boldsymbol{\rho}}=\left(\begin{array}{ccc}
0 & 0 & 0 \\
0 & 0 & 0 \\
0 & 0 & \left(\frac{\left(\tilde{q}^{0}\right)^{2}}{\tilde{q}^{2}}\right)
\end{array}\right)
$$

We have now reached a solid generalization point. Everything about the spin structure of the pure QED piece of the matrix element $\mathcal{M}_{\pi}^{\gamma}$ is quantified in $\tilde{\boldsymbol{\rho}}$. Furthermore, it is a simple matter to tell if the photons (virtual or otherwise) represent a pure state since $\frac{\operatorname{tr}\left(\tilde{\boldsymbol{\rho}}^{2}\right)}{\operatorname{tr}(\tilde{\boldsymbol{\rho}})}=1$ for a pure state. Moving on to unpolarized lepton scattering and defining a new density matrix $\boldsymbol{\rho}$, one has the following ${ }^{8}$

$$
\rho_{i j} \equiv f_{i j} \sum_{l s p i n s} \epsilon_{i}^{*} \epsilon_{j}
$$

The symbols $f_{i j}$ are as-yet-unspecified kinematic factors. The right hand side of Eqn. 2.23 can be written out explicitly (neglecting electron mass) given the definitions of $\epsilon_{\mu}$.

$$
\begin{aligned}
\sum_{\text {lspins }} \epsilon_{\mu}^{*} \epsilon_{\nu} & =\operatorname{tr}\left(\gamma_{\mu} \not k \gamma_{\nu} \not k^{\prime}\right) \\
& =4\left(k_{\mu} k_{\nu}^{\prime}+\frac{1}{2} q^{2} \eta_{\mu \nu}+k_{\mu}^{\prime} k_{\nu}\right)
\end{aligned}
$$

Evaluating this expression one can obtain the following relationships, which will end

\footnotetext{
${ }^{7}$ For real the real photon this appears to be singular but one is reminded that the real photon will never have longitudinal polarization and thus never suffer the singularity. The example is used as a sort of place holder for electroproduction.

${ }^{8}$ Note that the density matrices defined here are rigorously based on a true density matrix but frequently lack the normalization condition $\operatorname{tr}(\boldsymbol{\rho})=1$. This poses no problem here.
} 
up being of the utmost importance to the subject at hand.

$$
\begin{aligned}
& \sum \epsilon_{1}^{*} \epsilon_{1}=2 q^{2}\left(\frac{q^{2}}{|\mathbf{q}|^{2}} \cot ^{2} \frac{\theta_{e}}{2}-1\right) \\
& \sum \epsilon_{2}^{*} \epsilon_{2}=-2 q^{2} \\
& \sum \epsilon_{3}^{*} \epsilon_{3}=2 q^{2}\left(\frac{-\left(q^{0}\right)^{2}}{q^{2}}\right)\left(\frac{q^{2}}{2|\mathbf{q}|^{2}}\right) \cot ^{2} \frac{\theta_{e}}{2} \\
& \sum \epsilon_{1}^{*} \epsilon_{3}=\frac{4 q^{0}|\mathbf{k}|\left|\mathbf{k}^{\prime}\right|}{|\mathbf{q}|^{2}} \sin \theta_{e}\left(|\mathbf{k}|+\left|\mathbf{k}^{\prime}\right|\right)
\end{aligned}
$$

The object expressed by Eqn. 2.25 is symmetric and all components unconstrained by Eqn. 2.25 are zero. There should be some manipulation to write this as a density matrix, since there are common kinematic factors in the elements. Firstly, it is customary and convenient to define the parameter $\epsilon$.

$$
\epsilon \equiv\left(1+2 \frac{|\mathbf{q}|^{2}}{Q^{2}} \tan ^{2} \frac{\theta_{e}}{2}\right)^{-1}
$$

The definition allows one to express the elements in Eqn. 2.25 in a more compact way, without reference to the electron scattering angle.

$$
\begin{aligned}
& \sum \epsilon_{1}^{*} \epsilon_{1}=-2 q^{2} \frac{1+\epsilon}{1-\epsilon} \\
& \sum \epsilon_{2}^{*} \epsilon_{2}=-2 q^{2} \\
& \sum \epsilon_{3}^{*} \epsilon_{3}=2 q^{2}\left(\frac{\left(q^{0}\right)^{2}}{q^{2}}\right) \frac{\epsilon}{1-\epsilon} \\
& \sum \epsilon_{1}^{*} \epsilon_{3}=-2 q^{2} \sqrt{\frac{\left(q^{0}\right)^{2}}{4 Q^{2}}} \frac{[2 \epsilon(1+\epsilon)]^{\frac{1}{2}}}{1-\epsilon}
\end{aligned}
$$

One can now choose the factors $f_{i j}$.

$$
\begin{aligned}
& f_{11}=f_{22}=\frac{1}{-4 q^{2}}(1-\epsilon) ; \quad f_{33}=\frac{1}{-2 q^{2}}\left(\frac{q^{4}}{\left(q^{0}\right)^{4}}\right)(1-\epsilon) \\
& f_{13}=\frac{1}{-2 q^{2}}\left(\frac{Q^{2}}{\left(q^{0}\right)^{2}}\right)(1-\epsilon)
\end{aligned}
$$

These factors serve the purpose of constructing a density matrix with simple interpretation and will allow one to write the squared matrix elements for the process 
of interest directly in terms of the matrices. If one defines $\epsilon_{L} \equiv\left(\frac{Q^{2}}{\left(q^{0}\right)^{2}}\right) \epsilon$, then the density matrix can be written as follows [28].

$$
\boldsymbol{\rho}=\left(\begin{array}{ccc}
\frac{1}{2}(1+\epsilon) & 0 & -\left[\frac{1}{2} \epsilon_{L}(1+\epsilon)\right]^{\frac{1}{2}} \\
0 & \frac{1}{2}(1-\epsilon) & 0 \\
-\left[\frac{1}{2} \epsilon_{L}(1+\epsilon)\right]^{\frac{1}{2}} & 0 & \epsilon_{L}
\end{array}\right)
$$

This decomposition of the electron current gives the interpretation of the virtual photon as an incoherent mixture of pure states, as can be found from the density matrix in Eqn. 2.29. Note that in the extreme forward limit with $Q^{2} \rightarrow 0, \epsilon$ approaches zero ${ }^{9}$ and the density matrix is diagonal without a longitudinal component. It will be useful to write the density matrix as the sum of the squares of "polarization" vectors $\mathbf{b}_{1}$ and $\mathbf{b}_{2}$.

$$
\mathbf{b}_{1} \mathbf{b}_{1}^{\dagger}+\mathbf{b}_{2} \mathbf{b}_{2}^{\dagger}=\boldsymbol{\rho}
$$

Now that the insight of the density matrix has been obtained, one can write the electroproduction amplitude in terms of it. It is important to first rewrite the amplitude using the current conservation restrictions of Eqn. 2.13. In this way one will be able to see that the $3 \times 3$ size of the density matrix will suffice to parameterize the $4 \times 4$ leptonic tensor. In the center of mass frame previously discussed the momentum transfer vector can be written explicitly.

$$
q=\left(q^{0}, 0,0, q^{3}\right)
$$

From current conservation one can easily find a few useful relationships.

$$
\begin{aligned}
& q^{0} \epsilon^{0}-q^{3} \epsilon^{3}=0 \\
& q^{0} T^{0}-q^{3} T^{3}=0
\end{aligned}
$$

Recall that $\epsilon^{\mu}$ is the lepton current matrix element modulo the charge factor and

\footnotetext{
${ }^{9}$ In the limit $Q^{2} \rightarrow 0$ one must not neglect the electron mass even if the momenta are high. To get the limiting behavior of $\epsilon$ the expression with $m_{e}$ restored is to be used instead of the expression in the text.
} 
that $T^{\mu}$ is the hadronic current matrix element including the charge factor. With these facts the amplitude can be rewritten.

$$
\begin{aligned}
\epsilon_{\mu} T^{\mu} & =\left[\left(\left(\frac{q^{3}}{q^{0}}\right)^{2}-1\right) \epsilon^{3} T^{3}-\epsilon^{1} T^{1}-\epsilon^{2} T^{2}\right] \\
& =\left[\left(\frac{Q^{2}}{\left(q^{0}\right)^{2}}\right) \epsilon^{3} T^{3}-\epsilon^{1} T^{1}-\epsilon^{2} T^{2}\right]
\end{aligned}
$$

This makes the amplitude become:

$$
\mathcal{M}_{\pi}=\frac{e}{Q^{2}}\left[\left(\frac{Q^{2}}{\left(q^{0}\right)^{2}}\right) \epsilon^{3} T^{3}-\epsilon^{1} T^{1}-\epsilon^{2} T^{2}\right]
$$

The appropriate amplitude is now written strictly in terms of the spacial components of the current matrix elements. This means that the density matrix has enough components to specify the cross section, along with the additional benefit of the smooth link to photoproduction.

$$
\sum_{\text {spins }}\left|\mathcal{M}_{\pi}\right|^{2}=\left(\frac{2 e^{2}}{Q^{2}}\right) \frac{1}{1-\epsilon} \sum_{i j} \rho_{i j} \rho_{i j}^{H}
$$

The definition of the hadronic density matrix as used above should be evident.

$$
\rho_{i j}^{H} \equiv \sum_{h s p i n s} T_{i}^{*} T_{j}
$$

\subsubsection{Density Matrix and Rotations}

Now that the photon density matrix is properly defined one can examine the properties of this construction which will benefit the present study. It is useful to change the basis of the polarization vectors $\epsilon_{\lambda}^{\mu}$. The basis displayed in Eqn. 2.37 makes use of the circular polarization states along with a longitudinal state.

$$
\begin{aligned}
e_{+}^{\mu} & \equiv \frac{1}{\sqrt{2}}(0,-1,-i, 0) \\
e_{-}^{\mu} & \equiv \frac{1}{\sqrt{2}}(0,1,-i, 0) \\
e_{L}^{\mu} & \equiv\left(q^{0}, 0,0, q^{3}\right) / \sqrt{q^{2}}
\end{aligned}
$$


In the previous basis $\lambda$ took values in $\{x, y, L\}$ and the new basis $\lambda$ takes values in $\{+,-, L\}$. One can obviously write the virtual photon polarization vectors $\mathbf{b}_{1}$ and $\mathbf{b}_{2}$ in the new basis. This point will be demonstrated on a generic vector $\mathbf{b}$.

$$
\mathbf{b}=\sum_{\lambda} a_{\lambda} \mathbf{e}_{\lambda}
$$

One can then use the orthogonality conditions for the basis set defined in Eqn. 2.37 in order to specify the coefficients $a_{\lambda}$.

$$
a_{\lambda}=f_{\lambda} \mathbf{b} \cdot \mathbf{e}_{\lambda}^{*}
$$

Where $f_{\lambda}$ is positive for $\lambda$ in $\{L\}$ and negative otherwise but always of unit magnitude. Taking the " $\mathrm{z}$ " direction to be along the direction of the virtual photon, the transformation of $\mathbf{b}$ about the azimuthal angle of the electron plane is known [62].

$$
\begin{aligned}
& b^{1}(\phi)=\cos \phi b^{1}(0)-\sin \phi b^{2}(0) \\
& b^{2}(\phi)=\sin \phi b^{1}(0)+\cos \phi b^{2}(0)
\end{aligned}
$$

The decomposition used in Eqn. 2.38 will change as a function of azimuthal angle $\phi$ but in a convenient way.

$$
\begin{aligned}
& a_{+}(\phi)=e^{-i \phi} a_{+}(0) \\
& a_{-}(\phi)=e^{+i \phi} a_{-}(0) \\
& a_{L}(\phi)=a_{L}(0)
\end{aligned}
$$

These are precisely the transformations of the helicity states of a spin one object under spacial rotation. The benefit of transforming the density matrix into this basis is that it will be clear how each component of the density matrix transforms. The change of basis can be simply effected by writing the spacial part of the polarization vector $\mathbf{b}$ in terms of the new components.

$$
\tilde{\boldsymbol{b}}=\left(a_{+}, a_{-}, a_{L}\right)
$$


When this procedure is carried out on both the polarization vectors $\mathbf{b}_{1}$ and $\mathbf{b}_{2}$ that describe the density matrix, the new specification of the density matrix can easily be written.

$$
\tilde{\mathbf{b}}_{1} \tilde{\mathbf{b}}_{1}^{\dagger}+\tilde{\mathbf{b}}_{2} \tilde{\mathbf{b}}_{2}^{\dagger}=\left(\begin{array}{ccc}
\rho_{++} & \rho_{+-} & \rho_{+L} \\
\rho_{-+} & \rho_{--} & \rho_{-L} \\
\rho_{L+} & \rho_{L-} & \rho_{L L}
\end{array}\right)
$$

The components $\rho_{\lambda \lambda^{\prime}}$ transform as $a_{\lambda}^{*} a_{\lambda^{\prime}}$ under Lorentz transformations, in particular the azimuthal rotations which were previously discussed. This is an important development since, as Fig. 2.2 clearly shows, any general pion production kinematics can be reached from the planar kinematics $\left(\phi^{*}=0\right)$ by an azimuthal rotation of the electron density matrix. The $\phi^{*}$ dependence for specific linear combinations of the density matrix elements will turn out to be important.

$$
\begin{aligned}
\rho_{L L}\left(\phi^{*}\right) & =\rho_{L L}(0) \\
\left(\rho_{++}\left(\phi^{*}\right)+\rho_{--}\left(\phi^{*}\right)\right) & =\left(\rho_{++}(0)+\rho_{--}(0)\right) \\
\left(\rho_{+-}\left(\phi^{*}\right)+\rho_{-+}\left(\phi^{*}\right)\right) & =\left(\rho_{+-}(0)+\rho_{-+}(0)\right) \cos 2 \phi^{*} \\
\left(\rho_{+L}\left(\phi^{*}\right)+\rho_{L+}\left(\phi^{*}\right)\right) & =\left(\rho_{+L}(0)+\rho_{L+}(0)\right) \cos \phi^{*} \\
\left(\rho_{-L}\left(\phi^{*}\right)+\rho_{L-}\left(\phi^{*}\right)\right) & =\left(\rho_{-L}(0)+\rho_{L-}(0)\right) \cos \phi^{*}
\end{aligned}
$$

\subsubsection{Symmetry and Cross Sections}

Parity symmetry was used to write Eqn. 2.44 in such a concise form. In particular for an unpolarized reaction, the object $\boldsymbol{\rho}$ is symmetric and the phase transformations from azimuthal rotations stated in Eqn. 2.41 can be turned into trigonometric functions. A polarized measurement will have the density matrix $\boldsymbol{\rho}$ being of mixed symmetry with the antisymmetric portion being proportional to the measured helicity. Equation 2.45 shows how the longitudinal-transverse interference term behaves for an antisymmetric density matrix.

$$
\begin{aligned}
\left(\rho_{+L}\left(\phi^{*}\right)+\rho_{L+}\left(\phi^{*}\right)\right) & =-\left(e^{i \phi^{*}} \rho_{+L}(0)+e^{-i \phi^{*}} \rho_{L+}(0)\right) \\
& =-2 \rho_{+L}(0)\left(e^{i \phi^{*}}-e^{-i \phi^{*}}\right) \\
& =-4 \rho_{+L}(0) \sin \phi^{*}
\end{aligned}
$$


This analysis shows that an unpolarized cross section for a parity symmetric interaction will have the density matrix elements being even functions of the azimuthal angle. Adding polarization to the measurement will give the possibility of odd trigonometric functions as displayed in Eqn.2.45. For the current measurement the density matrix elements (and thus the cross section) will be an even function of $\phi^{*}$ with precisely the dependencies listed in Eqn. 2.44.

All that remains is to construct the pion production cross section written with its $\phi^{*}$ dependence explicit. It is a simple matter to add phase space differentials to the squared matrix element in order to obtain a cross section. Working (for the moment) in the laboratory frame and taking the symbol dLips to represent the Lorentz invariant phase space of the final state hadrons, one can write Eqn. 2.46.

$$
d \sigma=\frac{1}{4 E m_{p}}\left(\frac{d^{3} k^{\prime}}{(2 \pi)^{3} 2 E^{\prime}} d \text { Lips }\right)\left[\frac{1}{4} \sum_{\text {spins }}\left|\mathcal{M}_{\pi}\right|^{2}\right]
$$

The invariant phase space differential dLips is understood to include the 4-momentum conserving delta function and normalization factor $(2 \pi)^{4} \delta^{4}\left(p_{i}-p_{f}\right)$ where $p_{i}$ and $p_{f}$ are understood to represent the total initial and final 4-momentum respectively. This specification is fairly standard and has been used in many references [75]. The final electron integration measure can be unpacked so to produce the distribution in lab frame electron variables.

$$
d \sigma=\frac{1}{4 E m_{p}}\left(\frac{E^{\prime} d E^{\prime} d \Omega_{e}}{2(2 \pi)^{3}} d \text { Lips }\right)\left[\frac{1}{4} \sum_{\text {spins }}\left|\mathcal{M}_{\pi}\right|^{2}\right]
$$

Then the differential cross section can be written fairly simply.

$$
\frac{d \sigma}{d E^{\prime} d \Omega_{e}}=\frac{E^{\prime}}{64 \pi^{3} E m_{p}}(\text { Lips })\left[\frac{1}{4} \sum_{\text {spins }}\left|\mathcal{M}_{\pi}\right|^{2}\right]
$$

Making the density matrix substitution, one can then arrive at a new expression which can be matched more easily to photoproduction, with the density matrix as 
the connection point.

$$
\frac{d \sigma}{d E^{\prime} d \Omega_{e}}=\frac{\alpha}{8 \pi^{2}} \frac{E^{\prime}}{E} \frac{1}{m_{p} Q^{2}} \frac{1}{1-\epsilon}(d \text { Lips })\left[\frac{1}{4} \sum_{i j} \rho_{i j} \rho_{i j}^{H}\right]
$$

The indices on the density matrices now take values in the set $\{+,-, L\}$. This will not change the statement of Eqn. 2.35 because the transformation represented in 2.43 leaves that combination invariant so long as the new polarization basis vectors are normalized. It is useful to work out the phase space for real photon excitations as well.

$$
\begin{gathered}
d \sigma^{\gamma}=\frac{1}{2 \tilde{q}^{0} 2 m_{p}}(\text { LLips })\left[\frac{1}{4} \sum_{\text {spins }}\left|\mathcal{M}_{\pi}^{\gamma}\right|^{2}\right] \\
\frac{d \sigma^{\gamma}}{d \text { Lips }}=\frac{1}{2\left(W^{2}-m_{p}^{2}\right)}\left[\frac{1}{4} \sum_{i j} \tilde{\rho}_{i j} \rho_{i j}^{H}\right]
\end{gathered}
$$

It is thus intuitively obvious to even the most casual of observers that one can write the electroproduction cross section as a factor times the "virtual" photoproduction cross section, obtained by replacing in Eqn. 2.51 the real photon density matrix with the electron current density matrix previously derived.

$$
\begin{gathered}
\frac{d \sigma^{\gamma^{*}}}{d \text { Lips }} \equiv \frac{1}{2\left(W^{2}-m_{p}^{2}\right)}\left[\frac{1}{4} \sum_{i j} \rho_{i j} \rho_{i j}^{H}\right] \\
\frac{d \sigma}{d E^{\prime} d \Omega_{e} d \text { Lips }}=\frac{\alpha}{2 \pi^{2}} \frac{E^{\prime}}{E} \frac{\left(W^{2}-m_{p}^{2}\right)}{2 m_{p} Q^{2}} \frac{1}{1-\epsilon}\left(\frac{d \sigma^{\gamma^{*}}}{d \text { Lips }}\right)
\end{gathered}
$$

Equation 2.53 suggests the definition of the virtual photon flux $\Gamma$.

$$
\begin{gathered}
\Gamma \equiv \frac{\alpha}{2 \pi^{2}} \frac{E^{\prime}}{E} \frac{\left(W^{2}-m_{p}^{2}\right)}{2 m_{p} Q^{2}} \frac{1}{1-\epsilon} \\
\frac{d \sigma}{d E^{\prime} d \Omega_{e} d \text { Lips }}=\Gamma\left(\frac{d \sigma^{\gamma^{*}}}{d \text { Lips }}\right)
\end{gathered}
$$

Equation 2.54 is satisfactory so long as one stays away from the extreme forward direction and works with highly relativistic electrons which is the case of the present study. Due to the fact that the electrons are highly relativistic there is the added 
benefit that the helicity states stay approximately unchanged under boosts. Therefore the helicity labels of the virtual photon density matrix $\boldsymbol{\rho}$ remain unchanged in a frame related to the laboratory frame by a boost. This means that we can write the virtual photon cross section in the center of mass frame and retain the interpretation of each of its terms.

If one specializes to the virtual photon-proton center of mass frame for the hadronic variables in the cross section (though leaving the leptonic variables in the $\mathrm{lab}^{10}$ ) a simple form can be obtained for our 5-fold and 2-fold cross sections.

$$
\frac{d \sigma}{d E^{\prime} d \Omega_{e} d \Omega_{\pi}^{*}}=\frac{\alpha}{2 \pi^{2}} \frac{E^{\prime}}{E} \frac{\left(W^{2}-m_{p}^{2}\right)}{2 m_{p} Q^{2}} \frac{1}{1-\epsilon}\left(\frac{d \sigma^{\gamma^{*}}}{d \Omega_{\pi}^{*}}\right)
$$

Using the facts that were discovered about the $\phi^{*}$ dependence of the factors $\rho_{i j}$ one can break the center of mass photon cross section into pieces with definite $\epsilon$ dependence. This will also incidentally separate the transverse and longitudinal polarization states and their interferences.

$$
\frac{d \sigma^{\gamma^{*}}}{d \Omega_{\pi}^{*}}=\frac{d \sigma_{T}}{d \Omega_{\pi}^{*}}+\epsilon \frac{d \sigma_{L}}{d \Omega_{\pi}^{*}}+\epsilon \frac{d \sigma_{T T}}{d \Omega_{\pi}^{*}} \cos 2 \phi^{*}+\sqrt{2 \epsilon(1+\epsilon)} \frac{d \sigma_{L T}}{d \Omega_{\pi}^{*}} \cos \phi^{*}
$$

The differentials $d \sigma_{\beta}$, then, probe specific portions of the hadronic density matrix previously defined.

$$
\begin{aligned}
\frac{d \sigma_{T}}{d \Omega_{\pi}^{*}} & \propto\left(\rho_{++}^{H}+\rho_{--}^{H}\right) \\
\frac{d \sigma_{L}}{d \Omega_{\pi}^{*}} & \propto \rho_{L L}^{H} \\
\frac{d \sigma_{T T}}{d \Omega_{\pi}^{*}} & \propto-\left(\rho_{+-}^{H}+\rho_{-+}^{H}\right) \\
\frac{d \sigma_{L T}}{d \Omega_{\pi}^{*}} & \propto\left(\rho_{-L}^{H}+\rho_{L-}^{H}-\rho_{+L}^{H}-\rho_{L+}^{H}\right)
\end{aligned}
$$

The labels on these functions obviously refer to transverse (T), longitudinal (L), transverse-transverse interference (TT) and longitudinal-transverse interference (LT). Now one has a straightforward way to fit the angular dependence of the extracted

\footnotetext{
${ }^{10}$ This can be done because our designations of the virtual photon polarizations will not be strongly affected by this due to the small mass of the electron. Any kinematic factors will cancel in the relation between the 5 -fold and 2-fold differential cross sections.
} 
cross sections and find these functions (given some suitable expansion in the azimuthal angle). It will also become apparent in Chapter 10 that these functions can be explicitly written in terms of the CGLN multipoles defined in Section 1.5.3 and can thus be used to probe the multipoles at some level.

\subsection{Elastic Radiative}

When one identifies the backgrounds for the most important process to this work (pion electroproduction) one inevitably must discuss radiative processes. Far more will be learned about radiative events in Chapter 4. The current section concerns itself with elastic radiative processes which may "masquerade" as pion electroproduction processes in data analysis. The process of interest in this section is represented in Eqn. 2.58.

$$
e+p \rightarrow e^{\prime}+p^{\prime}+\gamma
$$

The kinematics for such a reaction will not be as easily aided by the one photon exchange approximation as the case of pure pion electroproduction in the center of mass was. The approach of this section is then to define kinematical variables first in a general way (with no implicit approximation) with basically only vectorial quantities defined. After that is concrete some important kinematical angles will be discussed and pictured in the context of the single photon exchange approximation. It should also be mentioned that multiple photon emissions could also have an important effect on calculated cross sections as the total radiated photon energy becomes very small. Luckily, the angular dependence of many-photon radiations will not be important and can essentially be integrated out in the low energy limit. This prevents one from having to work out the full kinematics of the n-body final state. The multi-photon effect will therefore only be discussed in Chapter 4.

In the case of single meson production with no radiation or pure elastic processes with no radiation the momentum and energy constraints on the phase space were of a simple nature. This effectively meant that we could use momentum and energy conservation prior to calculating the detailed phase space integrations that occur. In these cases it was also true that the angles and energies which were kept as the "free" variables in those processes were easily related to measured quanti- 
ties. For example, because of the center of mass description of pion production one was able to separate the the variables which describe the electron current, $Q^{2}$ and $W$ from those which described the hadron current, $\Omega_{\pi}$. The presence of radiation destroys this separation because $Q^{2}$ and $W$ are no longer enough to characterize the leptonic current, information is needed on the "free" variables corresponding to the radiated photon. What this means is that it is important to define all kinematic variables for the final state (for $n$ particles on shell there are $3 n$ of them) and carefully perform the phase space integrations in order to eliminate the unnecessary ones.

Figure 2.3 makes a consistent definition of all of the observed momenta for a radiative process. It is assumed that the observed momenta match the experimental definitions that will be espoused in this work. The initial and final state leptons are detected along with the initial and final state nucleons. The figure represents

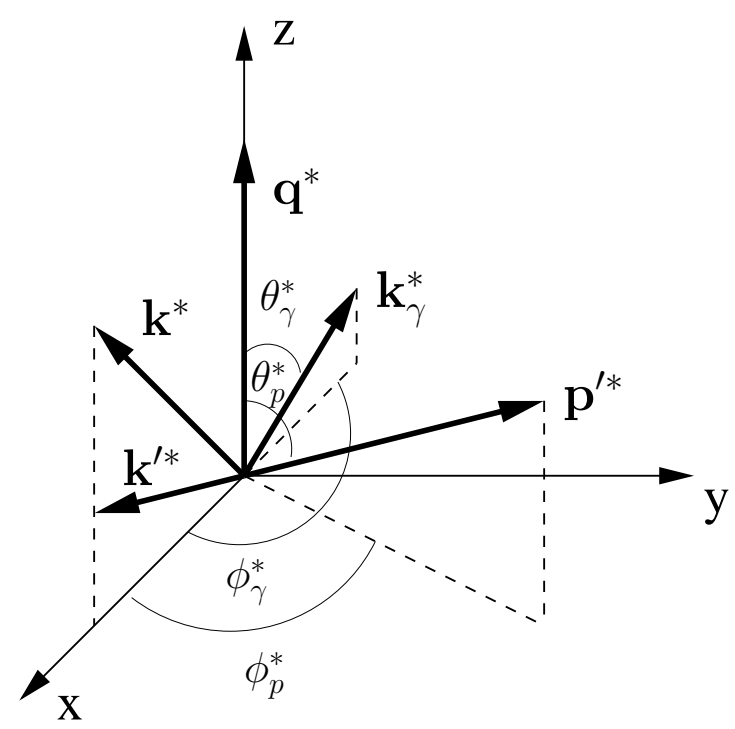

Figure 2.3: Kinematics of elastic radiative process.

the kinematics in the center of mass system described in the Section 2.2. Note that the previous center of mass frame is still well defined for the elastic and elastic radiative reactions, though may no longer correspond to the center of mass. Drawn in Fig. 2.3 are the measured kinematics and the $\mathbf{q}$ vector which is defined to be the subtraction of the incoming and outgoing leptons, $q \equiv k-k^{\prime}$. For radiative 
processes this need not be the momentum transfer to the hadronic system and therefore will not represent the value of momentum transfer used in the proton form factors to work out the elastic radiative dynamics. Nevertheless, the figure represents the kinematical variables that one wants to cast the final differential cross section in. This could force some algebraic manipulation during the integration over the photon momentum variables. The true momentum transfer to the hadronic current will be displaced from the " $\mathrm{z}$ " axis in the previous figure and will be needed to do computations for the dynamics ${ }^{11}$. The true momentum transfer (4-momentum of the

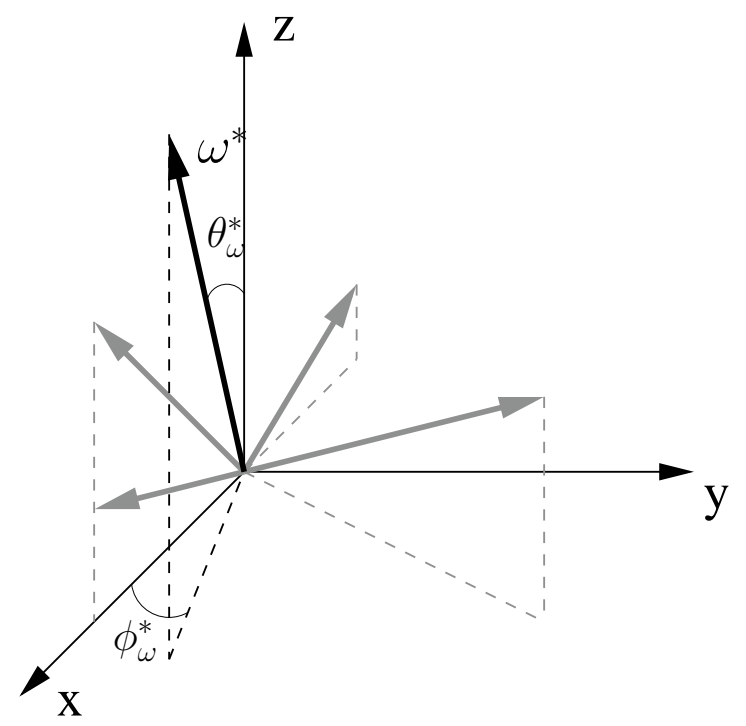

Figure 2.4: True momentum transfer to hadronic current.

exchanged hard photon) will be denoted as $\omega$ and a representation of the direction of this momentum is seen in Fig. 2.4. In the figure the silhouettes of the lepton momenta, proton momentum, and radiated photon momentum have been retained but their labels suppressed. The labels of the momenta can then be summarized somewhat succinctly for the center of mass frame variables.

$$
\begin{aligned}
& k=\left(E^{*}, \mathbf{k}^{*}\right) ; \quad k^{\prime}=\left(E^{* *}, \mathbf{k}^{\prime *}\right) ; \quad p=\left(E_{p}^{*}, \mathbf{p}^{*}\right) ; \\
& p^{\prime}=\left(E_{p}^{\prime *}, \mathbf{p}^{\prime *}\right) ; \quad k_{\gamma}=\left(E_{\gamma}^{*}, \mathbf{k}_{\gamma}^{*}\right)
\end{aligned}
$$

\footnotetext{
${ }^{11}$ Although it will be seen that this difference in momentum transfer can be ignored to first approximation if the radiated photon momentum is "soft."
} 
The lab frame counterparts to these variables are denoted by the convention in Appendix C. The true momentum transfer 4-vector $\omega$ should be defined piecewise because the definition depends on which side of the interaction the radiated photon comes from. This separation is only valid in the single photon exchange approximation.

$$
\omega= \begin{cases}k-k^{\prime}, & \text { hadron radiates; } \\ p-p^{\prime}, & \text { lepton radiates; }\end{cases}
$$

It is useful to think about the elastic radiative process and how it will correlate with the measured kinematic variables. As far as the computed kinematics for each event of the present work, it is assumed that one is working with a neutral pion production event. From the point of view of missing mass and energy restrictions (which will be discussed in Chapter 7), however, some events can look like pion production but really be elastic radiative events. Figure 2.5 displays this situation of radiation on the incoming and/or outgoing lepton leg. When one calculates the elastic radiative process or even if one just attempts to estimate these processes, the most salient feature will be the narrow cone in which the radiation emerges about the lepton lines. This is depicted in Fig. 2.5. From the figure one of the most important features of the data used in the current work is visible. That is, that elastic radiative events will correlated to small values of the pion polar angle $\phi$. This fact is also true in the center of mass system. This means that most, if not all, of the elastic radiative events can be eliminated by rejecting a narrow band about $\phi=0$. This fact will be very useful in Chapter 7 .

\subsection{Meson Production Radiative}

Processes which produce $\pi^{0}$ particles can also radiate a photon into the final state. Although the treatment of this process will involve the same principles as the treatment of elastic radiative processes, the application of this treatment must be different. Elastic radiative processes can simply be removed from the data with only logistical concerns relating to experimental resolutions and the ability to isolate the elastic processes. On the other hand treating the radiation processes which also have a $\pi^{0}$ produced is a different story. These processes represent important signal physics 


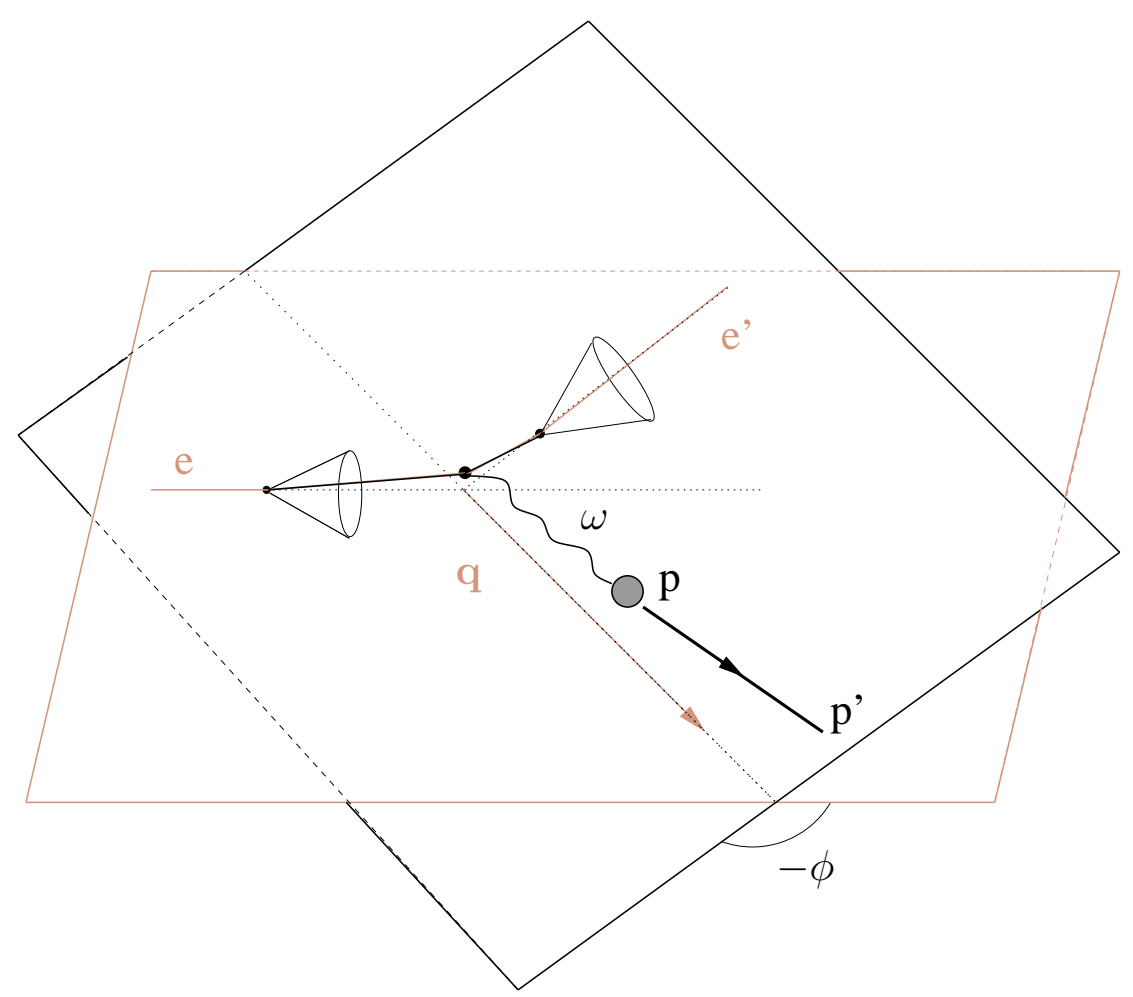

\section{Figure 2.5: Elastic radiative events and their projections onto the pion kinematics.}

which is foolish to throw away, indeed rejecting these events is even impossible if the radiated photon energy is less than the spectrometer resolution. One must then correct for these processes rather than subtract them. The details of this correction relating to the dynamics of physically measurable cross sections is postponed until Chapter 4 . Here only the kinematics of the radiative process are discussed.

$$
e+p \rightarrow e^{\prime}+p^{\prime}+\pi^{0}+\gamma
$$

As with the elastic radiative processes it is wise to retain the variables used to describe the non radiative process, although they may not have the same dynamical interpretation. In this spirit a picture of the kinematics of the process can be constructed. The picture is shown in Fig.2.6. The standard definition of the momentum transfer 4-vector, $q$, holds here. Once again the vectors in Fig. 2.6 are taken in the center of mass system described in Section 2.2. The initial and final 


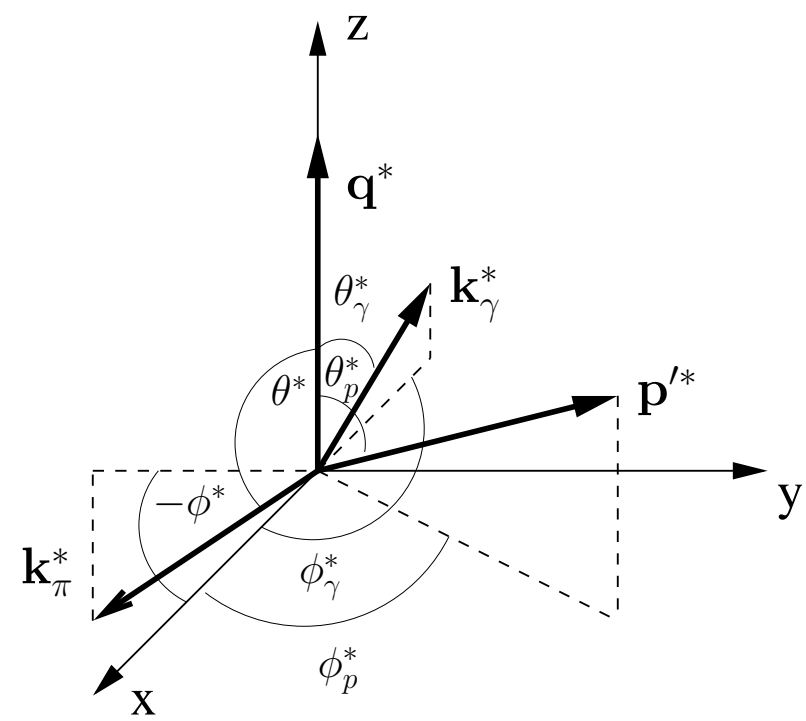

Figure 2.6: Kinematics of radiative meson production process.

lepton momenta are omitted from Fig. 2.6 since they are basically embodied in $\mathbf{q}^{*}$. Again, the kinematic variables can be summarized quite simply.

$$
\begin{aligned}
& k=\left(E^{*}, \mathbf{k}^{*}\right) ; \quad k^{\prime}=\left(E^{\prime *}, \mathbf{k}^{* *}\right) ; \quad p=\left(E_{p}^{*}, \mathbf{p}^{*}\right) ; \\
& p^{\prime}=\left(E_{p}^{\prime *}, \mathbf{p}^{\prime *}\right) ; \quad k_{\gamma}=\left(E_{\gamma}^{*}, \mathbf{k}_{\gamma}^{*}\right) ; \quad k_{\pi}=\left(E_{\pi}^{*}, \mathbf{k}_{\pi}^{*}\right) ;
\end{aligned}
$$




\section{CHAPTER 3 \\ Theoretical Issues}

\subsection{Theory Meets the Strong}

After the hard work of understanding the kinematics of electron scattering has been done, as in Chapters 1 and 2, one can begin to focus on the strong interaction physics. Though there is a quantum field theory which is supposed to predict the behavior of strongly interacting systems (QCD), it is difficult to solve, so insight will not come cheap. For this reason a multitude of simplifying calculations have been used to explain the phenomenon of electro-excitation of hadronic states. Some of these calculations have little to do with QCD other than the presence of "quark" objects and others are more directly inspired from the full structure of the QCD Lagrangian.

\subsection{Quark Model}

Despite our knowledge of QCD and the modern attempts to elucidate the spectrum of hadrons, the physics community still classifies baryons via the quark model [48]. This is not a bad thing, indeed the quark model provides the easiest classification scheme via symmetry properties of hadronic wave functions. There are also many dynamical predictions of the quark model which are remarkably accurate for a model which is supposed to be subsumed by the standard model and QCD. The quark model is so successful with a number of decay and mass predictions that QCD must explain why the quark model is true in many situations.

In this work one is interested in the dynamical couplings involved in $\gamma N \rightarrow \Delta$. Therefore, it is clear that one must go beyond classification type symmetries and delve into the actual mechanisms for the situation. Even still, it is important to know what is the standard classification scheme for the nucleon and delta multiplets. The needs of baryon spectroscopy dictate three distinct ways in which quark type models can be applied. Ideally one can use the marriage of these different branches to obtain

maximal information. Firstly, one can apply static symmetry groups which can be 
thought of as devoid of dynamics just to attempt to classify the baryon states which are observed ${ }^{1}$. Secondly one can use these "static" type symmetries to try to gain information about the relationship between different particle couplings, for example how $\pi^{+} n \Delta^{+}$vertexes constrain the properties of $\pi^{0} p \Delta^{+}$vertexes. Lastly, one can simply put actual quarks into a model potential and observe which bound states are borne out. Hyperfine splittings can then be added on to this bulk potential to effectively model the spectrum and simple electromagnetic interaction dynamics.

It is interesting to note that the founding work of Gell-Mann on the subject never endows "quarks" with any significant "reality" but just as triplet representations of $S U(3)_{f}$ symmetry multiplets [44]. Progress was even made obtaining and utilizing the baryon and meson mass-splittings in this non-dynamical approach $[71,72]$. Modern light baryon classification schemes deal with baryons containing $(u, d, s)$ quarks only. It is then assumed that each quark inside a baryon is a triplet representation of $S U(3)_{f}$. The empirical fact that each baryon has three quarks tells one how to construct the possible representations of $S U(3)_{f}$ in which the baryons transform. A good review of the detailed procedure of how this is worked out in group theory and a tutorial on the Young Tableaux formalism has been given elsewhere [48]. Using the $S U(3)_{f}$ symmetry one then has that (in group theory language):

$$
3 \otimes \mathbf{3} \otimes \mathbf{3}=\mathbf{1 0} \oplus \mathbf{8} \oplus \mathbf{8} \oplus \mathbf{1}
$$

The bold symbols stand for the representation of $S U(3)_{f}$ which has that number of states. These can also be called n-plets. For example the $\mathbf{3}$ in Eqn. 3.1 represents a basic triplet. The symbol $\otimes$ is the direct product and $\oplus$ represents the direct sum of groups of states. The easiest way to explain this is the simple example of a spin- $\frac{1}{2}$ object which transforms as an $S U(2)$ doublet. Coupling two spin- $\frac{1}{2}$ objects can result in total spin one (three states) or total spin zero (one state). This would be signified by $\mathbf{2} \otimes \mathbf{2}=\mathbf{3} \oplus \mathbf{1}$. In our baryon example it is easy to see that the $\mathbf{1 0}$ should be identified with the $\Delta$ decuplet and one of the $\mathbf{8}$ 's should be identified with the nucleon octet. From this application of flavor symmetry it is clear that should

\footnotetext{
${ }^{1}$ Though these symmetry groups will often use things like "spin" as a justification for use, the dynamics that "spin" would imply are more-or-less ignored.
} 
the quarks be endowed with reality in any model (like a potential or bag model) then they should be assigned fractional charges as displayed in Tbl. 3.1. The pure

\begin{tabular}{ccc}
\hline Quark Name & Quark Symbol & Charge (e) \\
\hline up & $\mathrm{u}$ & $\frac{2}{3}$ \\
down & $\mathrm{d}$ & $-\frac{1}{3}$ \\
strange & $\mathrm{s}$ & $-\frac{1}{3}$ \\
\hline
\end{tabular}

Table 3.1: Quark Charges.

flavor symmetry, however, cannot describe all of the states which are observed in baryon spectroscopy. For example, there are many more than 27 states expected to contain only combinations of the lightest three quarks. For this reason, and since it is well established that "partons" do exist and are of spin- $\frac{1}{2}$ nature $[18,39]$, one can extend the symmetry of the quark model to $S U(3)_{f} \times S U(2)=S U(6)$. This extension is equivalent to giving quarks a spin- $\frac{1}{2}$ nature and assuming that their spin orientations have no effect on their properties ${ }^{2}$. With this additional symmetry one is left with the situation displayed in Eqn. 3.2.

$$
6 \otimes 6 \otimes 6=56 \oplus 70 \oplus \mathbf{7 0} \oplus \mathbf{2 0}
$$

The Young tabulation techniques make the symmetry of these decomposed multiplets obvious so that the previous relation can be written as in Eqn.3.3.

$$
\mathbf{6} \otimes \mathbf{6} \otimes \mathbf{6}=\mathbf{5 6} \mathbf{6}_{S} \oplus \mathbf{7 0}_{M} \oplus \mathbf{7 0}_{M} \oplus \mathbf{2 0} \mathbf{0}_{A}
$$

The subscripts denote totally symmetric $(S)$, mixed-symmetric $(M)$ and totally anti-symmetric $(A)$ states with regard to permutation of any two flavor quantum numbers. Both the nucleon and the $\Delta$ multiplets are known to fit into the $\mathbf{5 6}_{S}$ along with 29 other particles which represent spin and/or flavor permutations of the three quarks. A final modification to this scheme is to include angular excitations since a three quark system need not have orbital angular momentum zero, in general. Then the total spin of the baryon will be produced by the orbital angular momentum and

\footnotetext{
${ }^{2}$ This is, of course, not true since hyperfine interactions can have a sizeable contribution to the baryon mass splittings.
} 
spin of the quarks.

$$
\begin{aligned}
\mathbf{J} & =\mathbf{L}+\mathbf{S} \\
P & \equiv(-1)^{l}
\end{aligned}
$$

The symbol " $P$ " defines the parity of the particle (or resonance) although this is only the naive expectation for the parity of the resonance in this case because dynamical effects are not taken into account in the relation between parity and angular momentum. Still this picture does quite well for predicting baryon multiplicity. The compact way of stating this addition to the model is that one has extended the required symmetry group to be $S U(6) \times O(3)$, where $O(3)$ is the familiar group of rotations in three dimensions. This makes the lowest particle multiplet, with $\left\{n-\right.$ plet,$\left.l^{P}\right\}$ representing the $S U(6)$ multiplet, orbital angular momentum and parity, a $\left\{\mathbf{5 6}_{S}, 0^{+}\right\}$which represents 56 states of zero orbital angular momentum and positive parity including the nucleon and $\Delta$ multiplets. If one respects this symmetry, then, it means that the nucleon and the $\Delta$ each are positive parity Swave states with equal mass. This is nearly reality and a good prediction for a model with no dynamics present. Many negative parity states in the $\left\{\mathbf{7 0}_{M}, 1^{-}\right\}$and D-wave states from $\left\{\mathbf{5 6}_{S}, 2^{+}\right\}$have been observed [48] and the predictions of the $S U(6) \times O(3)$-symmetric quark models seem to be at least partially displayed in nature.

It is clear that to go further and predict the mass splittings and other nonsymmetric properties of the baryon spectrum one must break the $S U(6) \times O(3)$ symmetry. Though this can be done in many different ways, some of the easiest to conceptualize are quark models which actually study the dynamics of bound states of three particles in a potential with endowed with "constituent" quark masses. In this picture symmetry breaking terms can simply be added to the potential. With this level of control the potentials can incorporate a variety of effects and can even be inspired by aspects of QCD. Indeed the migration from the classical symmetry descriptions to building block objects of the parton model to the formulation of current quarks as actual fermionic fields belonging to QCD began from exactly this sort of approach.

Because of the strong coupling nature of $\mathrm{QCD}$, quark mass operators are heav- 
ily renormalized and thus the mass of the quark fields (current quark masses) in QCD can be significantly lighter than the canonical 33\% of a baryon's mass. For this reason the constituent quark masses are defined to be much closer to this canonical percentage, since they are not modified by the interactions. In some sense, the use of constituent quark masses parameterizes a portion of the QCD interaction for light baryons. Examining a system of quarks endowed with constituent quark masses and a model potential will be the most fruitful method for predictions pertaining to this work. The appropriate classification type symmetries can easily be built into this model and are always included in the construction of baryon or meson wave functions. Another important restriction is consistency with the Pauli principle. Given these restrictions and all the appropriate model potentials quark models have come quite far in predicting the baryon spectrum [19]. One salient feature in many successful quark model calculations from non-relativistic to fully relativized approaches is a color hyperfine interaction inspired by one gluon exchange in QCD [49, 50, 45, 19]. The portion of the interaction Hamiltonian which represents the color hyperfine interaction for baryons is written in Eqn. 3.5.

$$
H_{h y p}^{i j}=\frac{2 \alpha_{s}}{2 m_{i} m_{j}}\left[\frac{8 \pi}{3} \delta^{3}\left(\mathbf{r}_{i j}\right) \mathbf{S}_{i} \cdot \mathbf{S}_{j}+\frac{1}{r_{i j}^{3}}\left(3 \mathbf{S}_{i} \cdot \hat{\mathbf{r}}_{i j} \mathbf{S}_{j} \cdot \hat{\mathbf{r}}_{i j}-\mathbf{S}_{i} \cdot \mathbf{S}_{j}\right)\right]
$$

The vector $\mathbf{r}_{i j}$ represents the separation between the $i^{\text {th }}$ and the $j^{\text {th }}$ constituent just as the symbols $\mathbf{S}_{i}$ and $\mathbf{S}_{j}$ represent their spins. The strong coupling constant is denoted by $\alpha_{s}$ and the shorthand $r_{i j}=\left|\mathbf{r}_{i j}\right|$ is used. This potential has been found by many models to be the key ingredient along with either a harmonic [49] or linear $[45,19]$ confining potential is used. Owing to the smallness of the spin-orbit forces [49] the color hyperfine interaction seems to be the term which is responsible for the mass splittings and possible orbital contaminations within this model ${ }^{3}$.

In relation to the multipole excitation of the $\Delta$ state in this work, the color hyperfine interaction plays a very significant role if the data is to be reconciled with a quark model. In the limit of exact $S U(6) \times O(3)$ symmetry the nucleon

\footnotetext{
${ }^{3}$ It is the point of view of some reputable modern references [19] that the smallness of the spin orbit terms compared to the hyperfine terms is a consequence of treating the system nonrelativistically. This is rectified in relativized models.
} 
and $\Delta$, as stated, belong to the $\left\{\mathbf{5 6}_{S}, 0^{+}\right\}$multiplet. Thus the transitions $E 2$ and $M 1$ are to be made between states with no orbital angular momentum. In this situation the E2 transition is forbidden and the $M 1$ transition is a pure spin-flip transition. So if the group symmetry is exact then $M 1$ must dominate [12] and $R_{E M}=0$. The current world data on the subject in Fig. 1.7 shows that this is very nearly the case and was an early success of the $S U(6) \times O(3)$ schemes. The mass splittings between nucleon and $\Delta$ multiplets make it clear that this symmetry must be broken in some way so the question is how can it be broken in such a way as to not contradict the validity of the current $R_{E M}$ data. The addition of the mentioned color hyperfine interaction provides a method of breaking this symmetry which leads to D-wave admixtures in the nucleon and $\Delta$ and a value of $R_{E M} \simeq-0.41 \%$ at the real photon point [50]. The calculation that revealed this value of $R_{E M}$ was made in a truncated harmonic oscillator basis that has been shown to induce sensitivity to the truncation energy (highest oscillator energy) [89, 30]. A similar value of $R_{E M} \simeq-0.21 \%$ has been obtained in a model where the basis is expanded and the dynamics are relativized [19]. Therefore one can see that quark models with appropriate symmetry breaking potentials are perfectly capable of obtaining small negative values for $R_{E M}$ which are roughly consistent with experiments. What is especially surprising in this picture is that this seems to remain valid even when the photon virtuality is taken out to $\sim 6 \mathrm{GeV}^{2}$ ! Naively one would never expect this situation when probing physics on a scale roughly a sixth of the size of a proton since many of the quark model concepts like using "constituent" masses and absorbing some QCD effects into model potentials seem like they should depend on observations at a momentum scale corresponding to roughly the size of baryons.

\section{3 pQCD Predictions}

One of the most important discoveries relating to the strong interaction and our detailed understanding of QCD is that of asymptotic freedom [47]. Asymptotic freedom means that the renormalized coupling constant of QCD, $g$, has a stable UV fixed point at $g=0$ in the renormalization group flow which represents a free 
field theory ${ }^{4}$. This fact is true of Yang-Mills theories in general. The implication is that at infinite momentum (compared to the renormalization scale $\Lambda_{Q C D}$ ) QCD becomes just a free field theory of quarks and gluons. In particular, the so-called "beta" function ${ }^{5} \beta(g)$ can be calculated in QED and compared to Yang-Mills theories. The beta function controls the behavior of the interaction as one approaches higher momenta, if $\beta(g)$ is negative the coupling will decrease at high momenta and conversely. The fact that $\beta(g)$ is negative for Yang-Mills theories shows that the coupling constant for these theories, QCD in particular, becomes small in the vicinity of the previously mentioned fixed point. This allows a perturbative treatment in this momentum regime [76]. These facts make possible rigorous predictions of QCD which should be borne out in experiment with "large enough" momentum transfer at each quark-gluon vertex. The problem is simply the vague notion of "large enough" momenta, which means that there is no rigorous prediction for when these UV approximations should start to become close to reality.

After these ground breaking realizations about QCD, it was important to make a more solid connection with currently possible scattering experiments which essentially only probe quarks which are embedded in hadrons. Some of the most interesting testable predictions began when the abstract notions of the previous paragraph were applied to exclusive reactions in a way that focused on the calculational promise of pQCD [60]. A typical calculation of an $\mathrm{N}-\Delta$ magnetic transition form factor in pQCD can be represented as in Eqn. 3.6.

$$
G_{M}^{*}\left(Q^{2}\right)=\int_{0}^{1}[d x] \int_{0}^{1}[d y] \phi_{\Delta}^{*}\left(y_{i}, \tilde{Q}_{y}\right) T_{H}\left(x_{i}, y_{i}, Q^{2}\right) \phi_{N}\left(x_{i}, \tilde{Q}_{x}\right)\left[1+\mathcal{O}\left(\frac{m^{2}}{Q^{2}}\right)\right]
$$

The symbols $x_{i}$, and $y_{i}$ represent the fractions of the hadronic momenta carried by the quarks in the nucleon and $\Delta$ respectively. Obviously these must satisfy $\sum_{i=1}^{3} x_{i}=1$. The functions $\phi_{N}$ and $\phi_{\Delta}^{*}$ are the amplitudes for finding the nucleon and $\Delta$ in their three quark valence states respectively. One also has the definitions $\tilde{Q}_{x} \equiv \min _{i}\left(x_{i} Q\right)$ and $[d x] \equiv d x_{1} d x_{2} d x_{3} \delta\left(1-\sum_{i} x_{i}\right)$. Finally, the amplitude $T_{H}$ is the

\footnotetext{
${ }^{4}$ The coupling constant $g$ is analogous to the electric charge $e$ of QED and can be converted into a fine-structure type constant for QCD as $g^{2}=4 \pi \alpha_{s}$.

${ }^{5}$ Here $g$ is a generic coupling constant, using $\alpha_{s}$ will imply that QCD is being referred to.
} 
hard scattering amplitude which is calculable by normal perturbative techniques for the $3 q+\gamma^{*} \rightarrow 3 q$ process. The overview of the process is that the nucleon finds itself in a collinear configuration with probability parameterized by $\phi_{N}$, this configuration then participates in a hard scattering process into another collinear quark configuration and finally that quark configuration reforms into a $\Delta$ with probability parameterized by $\phi_{\Delta}$. Detailed calculation is beyond the scope of this survey but a few facts can be observed immediately. The hard scattering amplitude $T_{H}$ can be represented by diagrams of the type displayed in Fig. 3.1. The momentum transfered to the hadronic system by the photon must be transfered to one quark and then subsequently transfered to the other two. Each of the gluon exchanges come with a factor of $Q^{2}$ since all of the quarks must begin and end the hard scattering in a collinear configuration. The asymptotic behavior of $G_{M}^{*}\left(Q^{2}\right)$ is then governed by these gluon exchanges.

$$
G_{M}^{*}\left(Q^{2}\right) \propto\left(\frac{\alpha_{s}\left(Q^{2}\right)}{Q^{2}}\right)^{2}
$$

If one remembers that the strong coupling constant runs like logarithms of $Q^{2}$ then it is clear that the dominant asymptotic behavior of the magnetic form factor is $\frac{1}{Q^{4}}$. This variation is roughly equivalent to the variation of the dipole form factor $G_{D}=$ $\frac{1}{\left(1+\frac{Q^{2}}{0.71}\right)^{2}}$, and therefore if one observes experimentally that the quantity $\frac{G_{M}^{*}}{G_{D}} \sim$ const. it would be a possible hint of pQCD dominated behavior. Figure 1.9 shows that for previous measurements this situation is emphatically not borne out and that it appears that soft processes are still important up to $\sim 6 \mathrm{GeV}^{2}$. As briefly discussed

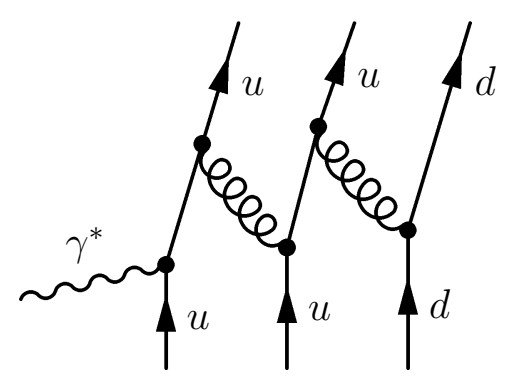

Figure 3.1: pQCD hard transition amplitude for $\Delta$ excitation. 
in Section 1.6, helicity conservation is a hallmark of perturbative QCD. At large momentum transfers the mass of the quarks effectively decrease or are renormalized to zero and therefore the chiral symmetry of QCD is exact at these momentum scales. Massless vector bosons can never transfer helicity to massless fermions and therefore in the pQCD picture hadron helicity must be conserved (since hadron helicities are composed of quark helicities). Therefore (as was previously stated) the amplitude $A_{\frac{3}{2}}$ must vanish in the large $Q^{2}$ limit. This forces $R_{E M}$ to unity. It is unlikely, however, that one will ever actually measure a value of unity for this parameter since this occurs in the $Q^{2} \rightarrow \infty$ limit. At higher $Q^{2}$ the data may move closer to this value but the parameter is still small and negative at $Q^{2} \sim 6 \mathrm{GeV}^{2}$. The question then becomes one of interpolating behavior for which interesting conjectures have been made [20]. The essential features of the arguments are that the asymptotic

smallness of the amplitude $A_{\frac{1}{2}}$ can slow the approach of $R_{E M}$ to unity such that the current experimental data are not completely unexpected even if pQCD makes a significant contribution to the large $Q^{2}$ behavior. In this picture the ratio $R_{E M}$ may cross zero somewhere between 4 and $10 \mathrm{GeV}^{2}$ while remaining small and negative before this cross over point.

\subsection{Phenomenological Analysis}

Though the previous sections have shown that there is some hope of getting rigorous theoretical input from QCD, it is unlikely that one will be able to predict all the amplitudes necessary in all kinematic regions to describe meson production off of nucleons as a whole. More likely the theory focuses on a single channel, like the resonant channel, via transition amplitudes. All of these specific predictions can be related to the pure $E_{l \pm}, M_{l \pm}$, and $S_{l \pm}$ meson production amplitudes. It can be seen, however, that any connections drawn between these amplitudes and a particular physical parameter (form factor maybe) might need some assumptions. It would be nice to simply extract all of the meson production multipoles which would make the connection between rigorous theoretical predictions and experiment free of assumptions. Unfortunately, it is not clear which multipole amplitudes are the most important for in a particular invariant energy region for all values of the momentum 
transfer $Q^{2}$. In order to compare with experiment one must then use phenomenological models with free parameters which represent the physical parameters of interest (like a $\Delta$ excitation form factor for example). These simpler models will provide the ability to fit experimental data and extract a physical parameter to compare with a presumably more fundamental calculation from QCD. The price that is paid for the use of such tools is possible model dependence. The numbers obtained from an experiment may have some ambiguity in them related to the reliability of the model used to extract them. The goal of such models is simply to be realistic but not as complicated as QCD which has, in principle, no free parameters. 


\section{CHAPTER 4 \\ Dynamics of Physics Backgrounds}

\subsection{Backgrounds and Interferences}

In order to reliably extract the parameters that are to be measured one must have a solid understanding of the dynamics of all physical processes involved in an experiment. The kinematical considerations covered in Chapter 2 clearly specify (through momentum and energy conservation) the mutual constraints that variables place on one another ${ }^{1}$. In order to extract specific observables in the presence of other uninteresting processes it is often not enough to know only these constraints. Therefore one must do as well as one can to predict how the phase space of each of these uninteresting processes is populated. For example, in some cases the background processes are exactly calculable like lepton current radiation. The calculation of radiation from a high energy lepton current will show that the radiated photons emerge in a very tight angular cone. This dynamical fact can be utilized in analysis. One must also have a solid understanding of which uninteresting processes are "coherent" and which are "incoherent." Additional coherent processes have precisely the same final state (though possibly different phase space population) and therefore must interfere with the process of interest. These should be treated with care and one must either be sure to be examining a region of phase space where these other coherent processes make negligible contribution or include interference parameters in the extraction. On the other hand the incoherent processes, if understood, can be subtracted straightforwardly ${ }^{2}$. There are still other processes which can be termed "quasi-coherent" processes which, from the point of view of quantum mechanics do not add at the amplitude level to the signal process and yet still cannot be separated from the signal processes, even in principle. Examples of such situations are soft photon radiative events. If one is to make concrete conclusions about the parameters of interest, each of these types of processes must be fully understood

\footnotetext{
${ }^{1}$ Known as phase space.

${ }^{2}$ Although experimental resolution can make this subtraction difficult at times.
} 
and in control.

\subsection{Elastic Radiative}

The kinematics for the elastic radiative interactions have been discussed in Section 2.3. The elastic radiative events are events which come from the reaction $e+p \rightarrow e^{\prime}+p^{\prime}+\gamma$, with a photon radiated either before or after the primary momentum transfer vertex. The missing mass for this work is always calculated by summing the 4-momenta of the incoming and outgoing measured particles. With the standard kinematic conventions one has that $p_{m}^{2}=\left(k+p-k^{\prime}-p^{\prime}\right)^{2}$. For elastic radiative events with a single radiated photon the missing mass is zero. These processes are an extremely important to understand because the low mass of the $\pi^{0}$ makes it difficult to separate from processes which have $p_{m}^{2}=0$. This is of course because of experimental resolution effects on the undetected invariant mass ${ }^{3}$.

One is then required to calculate or approximate in some way the contribution of processes which include an extra photon in the final state. Actually, one will find that the situation is made more complicated by the so called infrared divergence of QED. When the emitted photon energy is extremely low one calculates an infinite amplitude and other nonsensical features. The way to deal with these problems has, thankfully, been worked out and given a greater understanding of photon radiation. The procedure is:

1. Include next to leading order Feynman diagrams in the fine structure constant $\alpha$. These diagrams will have a similar and exactly cancelling IR divergence and render the perturbation series usable again.

2. For extremely low radiated energies one must re-sum the perturbation series including emission of any number of photons. This will essentially modify the predictions by an overall pre-factor.

The procedure means that one must, in general, consider radiation of many different

\footnotetext{
${ }^{3}$ The undetected invariant mass is computed experimentally by summing the energy and momentum present in the detectors and subtracting from the incident energy and momentum, the resulting 4 -vector is $p_{m}^{\mu}$. Sometimes in this work the variable $M_{x}^{2} \equiv p_{m}^{2}$ will be used to denote missing mass.
} 
numbers of photons despite the fact that they are supposed to be "small" since they come with more factors of $\alpha$ for more radiated photons. To be clear this would include calculating all of the following processes.

$$
e+p \rightarrow e^{\prime}+p^{\prime}+n \gamma
$$

It turns out that for the low energy photon limit (the only region of phase space where this re-summation is necessary) each of these processes are closely related to one another, so that we are only required to calculate single photon radiation [94].

It is important to be specific about the physical process one wishes to predict especially in the case of radiative events. Here the concern is for the cross section which includes an outgoing proton and electron. This will obviously include both elastic events and elastic with any amount of radiation in the final or initial states. Assuming the experimenter only has accurate particle identification on the detected particles $^{4}$, one can then come up with all the observed distributions. It is clear that in measuring elastic scattering one needs the tree-level theoretical prediction plus a soft bremsstrahlung contribution [65]. For the inelastic scattering contribution, i.e. with the mass of the hadronic system greater than $m_{p}$, a hard photon radiation should also be included [68]. In the end the cross section which is measured can be written as follows.

$$
\frac{d \sigma^{m e s}}{d \Omega_{e}}=\frac{d \sigma^{e l}}{d \Omega_{e}}+\int \frac{d^{3} k}{(2 \pi)^{3} 2 E_{\gamma}} \frac{d \sigma^{\gamma}}{d \Omega_{e} d E_{\gamma} d \Omega_{\gamma}}
$$

The $d \sigma^{\gamma}$ refers to the single photon emission process from an otherwise elastic process. The integral in Eqn. 4.2 should be taken over all the photon phase space. It should be mentioned that even calculation of "pure elastic" cross sections which are to be compared with experiment should contain the second term in Eqn. 4.2 but with a low photon energy cut-off corresponding to the resolution of the detector or, rather, its ability to reject events which have an extremely soft radiated

\footnotetext{
${ }^{4}$ In the meson production experiment discussed in this work particle identification rejections will also be used for the missing 4-momentum. This type of filtration will depend heavily on the process being measured, the extent to which that process is separable from background, and the resolutions of the measured parameters. It is best not to bias initial background calculations with this type of correlated filtration.
} 
photon. In the low energy photon limit the $d \sigma^{\gamma}$ term will have an IR divergence exactly canceled by the $\mathcal{O}\left(\alpha^{2}\right)$ and $\mathcal{O}(\alpha)$ interference contribution to $d \sigma^{e l}$. Since this IR divergence cancellation is absolutely necessary and the rest of the integral in Eqn.4.2 is dependent only on experimental conditions, many authors separate this term into soft and hard contributions. It should also be mentioned that while the form 4.2 is convenient in order to lay out the theoretical underpinnings of radiative events, it is not convenient for predicting what is seen in real detectors for exclusive processes since the integration in the second term on the right hand side should include detector acceptance effects. In those experimental situations the differential cross sections $d \sigma^{e l}$ and $d \sigma^{\gamma}$ can be used in conjunction with a Monte Carlo method to effectively perform the integration in the equation, with the detector properties folded in. Chapter 1 dealt with the lowest order elastic scattering. The single diagram that is needed for such a calculation is shown in Fig. 4.1 and the cross section thus computed will be referred to as $\frac{d \sigma^{e l}}{d \Omega_{e}}$. Recalling the analysis of Section 1.2 it

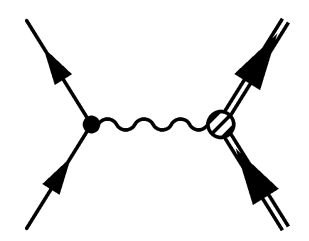

Figure 4.1: Leading order elastic electron proton scattering.

is clear that the leading order cross section (as well as all cross sections computed in this section) will depend on the Dirac and Pauli form factors $F_{1}$ and $F_{2}$. To calculate the elastic cross section including radiation of hard and soft photons two matrix elements need to be calculated. Firstly one should calculate the matrix element for emission of a single radiated photon $\left(e p \rightarrow e^{\prime} p^{\prime} \gamma\right), \mathcal{M}_{\gamma}$, to leading order. This will involve the mentioned IR divergence. Next one must calculate the pure elastic $\left(e p \rightarrow e^{\prime} p^{\prime}\right)$ amplitude $\mathcal{M}_{e l}$ consistently to $\mathcal{O}\left(\alpha^{2}\right)$ in order to prepare for the IR divergence cancellation. It is easy to relate the matrix elements to the cross 
sections denoted in Eqn. 4.2.

$$
\begin{aligned}
\frac{d \sigma^{e l}}{d \Omega} & \propto\left|\mathcal{M}_{e l}\right|^{2} \\
\frac{d \sigma^{\gamma}}{d \Omega_{e} d \Omega_{\gamma}} & \propto\left|\mathcal{M}_{\gamma}\right|^{2}
\end{aligned}
$$

The only thing missing is then to create a procedure which regulates IR divergent integrals that are guaranteed to come up. One popular method for dealing with this is to give the photon a small non-zero mass $m_{\gamma}$ and hope the final results are independent of this parameter.

The way to begin calculating these elastic radiative effects is to lay out each Feynman diagram which should be computed. Though it has been mentioned that calculating the amplitude contributions from single photon radiation will not be sufficient, they must still be included and represent the next to leading contribution to elastic radiative scattering. Figure 4.2 clearly depicts all single photon processes. The hadronic currents are drawn with a double line and leptonic with a single.

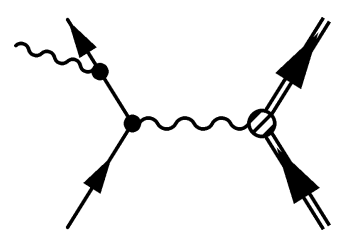

a)

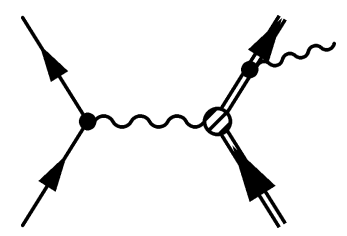

c)

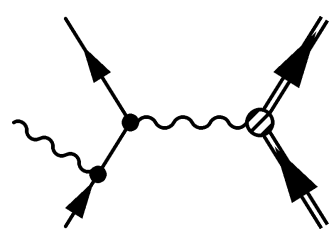

b)

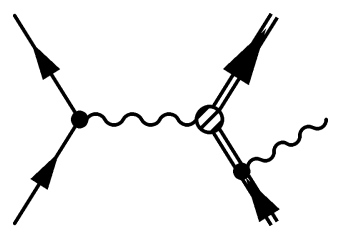

d)

Figure 4.2: $\mathcal{O}\left(\alpha^{\frac{3}{2}}\right)$ amplitude corrections.

For the pure elastic calculation the $\mathcal{M}_{e l}^{(1)}$ is a trivial calculation and is written schematically in Eqn. 1.1. The next contributions to the pure elastic matrix element will be denoted $\mathcal{M}_{e l}^{(2)}$. These contributions will include four electromagnetic vertices. Figure 4.3 shows each amplitude contribution at this order. Only the di- 


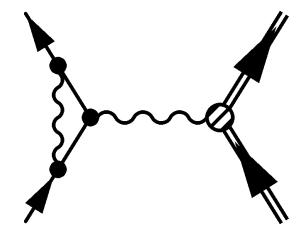

a)

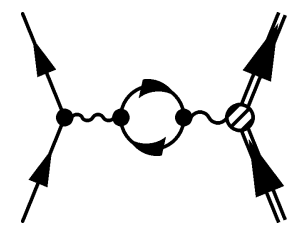

c)

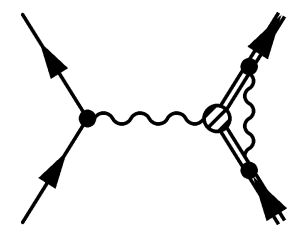

b)

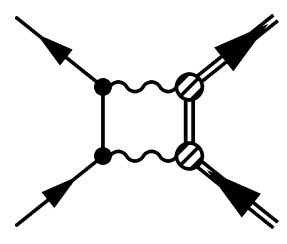

d)

Figure 4.3: $\mathcal{O}\left(\alpha^{2}\right)$ amplitude corrections.

agrams which only include pure QED corrections are straightforward to calculate. The others are complicated by the fact that the Dirac and Pauli form factors, though they parameterize the interaction in a general way, are no substitute for a perturbative expansion where they would be calculable in the strong coupling constant to appropriate order. This means that in order to calculate diagrams like those appearing in Fig. $4.3 \mathrm{~b}$ ) and d), one must use measured values for $F_{1}\left(q^{2}\right)$ and $F_{2}\left(q^{2}\right)$ and make some assumptions about how off-shell nucleons propagate. The measured values have considerable uncertainty and definitely do not span the range of photon virtuality while assumptions about off-shell nucleons tend to be complicated or drastic. The option is then simple, ignore them or calculate with an approximate model. It may be argued that these are much less important than the other diagrams, but one should still remember that the contribution from Fig. 4.3 b) is necessary to cancel the IR divergences in the diagrams depicted in Fig.4.2 b) and d).

Discussing all the theoretical aspects of elastic radiative events is beyond the scope of this work. The reference of R. Ent et al. takes up the task of producing a reliable method to calculate radiative effects for exclusive experiments using Monte Carlo methods [36]. Since one is typically not interested in measuring the outgoing photons some clever methods can be used to be sure that a Monte Carlo simulation has the correct "missing" energy dependence even though one may not be using the 
exact differential cross section for radiation of an extra photon into the final state. The reference deals with all of the theoretical issues previously discussed. The ability to make the mentioned calculations ensuring the correct energy dependence is based on the fact that the radiative reaction is strongly peaked such that the radiated photons travel along nearly the same direction as the incident and scattered electron and scattered proton. This fact has been previously mentioned and pictured in Fig. 2.5 for the lepton current.

If one assumes that the radiated photons are soft compared to the energies of the incoming and outgoing leptons and protons then one can compute the amplitudes of Fig. 4.2, which turn out to be proportional to the first order elastic scattering amplitude, $\mathcal{M}_{e l}^{(1)}$, in this approximation.

$$
\begin{aligned}
& \mathcal{M}_{\gamma}^{(a)}=e \mathcal{M}_{e l}^{(1)}\left(\frac{\epsilon \cdot k}{k_{\gamma} \cdot k}\right) \\
& \mathcal{M}_{\gamma}^{(b)}=e \mathcal{M}_{e l}^{(1)}\left(\frac{-\epsilon \cdot k^{\prime}}{k_{\gamma} \cdot k^{\prime}}\right) \\
& \mathcal{M}_{\gamma}^{(c)}=-e \mathcal{M}_{e l}^{(1)}\left(\frac{\epsilon \cdot p}{k_{\gamma} \cdot p}\right) \\
& \mathcal{M}_{\gamma}^{(d)}=-e \mathcal{M}_{e l}^{(1)}\left(\frac{-\epsilon \cdot p^{\prime}}{k_{\gamma} \cdot p^{\prime}}\right)
\end{aligned}
$$

The labels for the radiative amplitudes in Eqn. 4.4 are made to match the diagrams of Fig. 4.2. The expressions in Eqn. 4.4 are evaluated using the elastic kinematics for a given $Q^{2}$, this is approximately correct for a soft photon radiation. After this approximation several steps are used in the reference to come up with an IR finite cross section which can be used to generate Monte Carlo events.

The approximate steps which lead to a fruitful Monte Carlo simulation for elastic radiative events are outlined here in the order they are used in reference [36].

1. The angular distribution for the radiative process are very peaked around the directions of the external particle which emitted the photon. Schematically, one can take the angular distribution as the following.

$$
A\left(\Omega_{\gamma}\right)=\lambda_{e} \delta\left(\hat{\mathbf{k}}_{\gamma}-\hat{\mathbf{k}}\right)+\lambda_{e^{\prime}} \delta\left(\hat{\mathbf{k}}_{\gamma}-\hat{\mathbf{k}}^{\prime}\right)+\lambda_{p^{\prime}} \delta\left(\hat{\mathbf{k}}_{\gamma}-\hat{\mathbf{p}}^{\prime}\right)
$$


This is known as the "peaking approximation." The factors $\lambda_{e}, \lambda_{e^{\prime}}$ and $\lambda_{p^{\prime}}$ parameterize the angular distribution and are related to the angle integrals over each contribution which is peaked about the incident electron, outgoing electron and outgoing proton vectors respectively. The $\delta$-functions in Eqn. 4.5 simply pick out the appropriate angles and integrate to unity over the entire photon angle space $\Omega_{\gamma}$.

2. By integrating over all angles the energy dependence is constrained and the IR divergences are cancelled. Multiple low-energy photon radiations are taken into account in a similar fashion to Yennie et al. [94]. By using the parameter $\Delta E_{m}$ as the maximum total radiated energy one obtains Eqn. 4.6.

$$
\frac{d \sigma^{\text {meas }}}{d \Omega_{e}}\left(\sum_{i} E_{\gamma_{i}}<\Delta E_{m}\right)=\frac{d \sigma^{e l}}{d \Omega_{e}}\left(1-\delta_{\text {hard }}\right) e^{-\delta_{\text {soft }}\left(\Delta E_{m}\right)}\left[1+\mathcal{O}\left(\alpha^{2}\right)\right]
$$

The $E_{\gamma_{i}}$ are the individual photon energies and $\delta_{\text {hard }}$ parameterizes the radiative correction and IR cancellation along with $\delta_{\text {soft }}$.

3. Put the energy dependence and the peaked angular distribution into the calculation of a weight factor for a Monte Carlo event. For each elastic event pick a "leg" to radiate on and select a value for the radiated energy. Apply the weight factor based on the generated energy for the radiated photon and adjust the outgoing momentum 4-vectors to be consistent with energy conservation. The energies for the radiated photons are selected so as to produce the appropriate energy dependence.

Armed with this procedure one gains both an intuition of where radiative events will be seen (strongly peaked) and a means by which to simulate them. In this work both of these benefits will be utilized by removing most of the radiative events with a data restriction and verifying this with simulation.

\subsection{Meson Radiative}

Radiative processes do not only effect elastic scattering cross sections but the meson production mechanisms of interest in this work as well. The electromag- 
netic structure of these real photon emissions and vertex corrections are similar on the leptonic current side but more complicated on the meson production side, with the possibility of dependence on many more form factors than just the elastic radiative effects. Another key difference in the treatment of these radiative processes as opposed to the elastic radiative processes treated in Section 4.2 is that they more directly relate to the signal process. In Section 4.1 these processes were termed "quasi-coherent." The reason for this mostly relates to the special nature of massless particle radiation and IR divergences. In a quantum mechanical sense the one photon radiative processes which attach themselves to meson production as in Eqn. 4.7 do not add coherently (at the amplitude level) to the "pure" meson production process in Eqn. 4.8.

$$
\begin{gathered}
e+p \rightarrow e^{\prime}+p^{\prime}+\pi^{0}+\gamma \\
e+p \rightarrow e^{\prime}+p^{\prime}+\pi^{0}
\end{gathered}
$$

Even though the single photon (and multi-photon) radiations do not add coherently, the physical measured cross section still includes both processes since any real detector has an energy threshold below which one cannot detect an extra radiated photon. Then, though quantum mechanics says that these processes add incoherently and are in some sense "separate," they must both be included to obtain any physically measurable cross section. The symbol $\sigma^{m}$ will be used to represent the meson production process without radiation and the symbol $\sigma^{m \gamma}$ will represent the meson cross section including a single photon radiation. The symbol $\sigma_{m}^{\text {mes }}$ will mean the measured meson production cross section. Experimentally, this situation would allow the rejection of reasonably hard photon radiations since by measuring both the outgoing electron and proton one can enforce the missing momentum constraint $p_{m}^{2}=m_{\pi}^{2}$. The constraint allows one to (within resolutions) limit the maximum energy of the radiated photon. If one assumes that the maximum energy photon which can masquerade as a single pion event is $\Delta E$, then it is easy to see what cross 
section is actually being measured by the data extraction.

$$
\frac{d \sigma_{m}^{m e s}}{d \Omega_{e} d E^{\prime} d \Omega_{\pi}}=\frac{d \sigma^{m}}{d \Omega_{e} d E^{\prime} d \Omega_{\pi}}+\int_{0}^{\Delta E} \frac{d^{3} k}{(2 \pi)^{3} 2 E_{\gamma}} \frac{d \sigma^{m \gamma}}{d \Omega_{e} d E^{\prime} d \Omega_{\pi} d E_{\gamma} d \Omega_{\gamma}}
$$

Equation 4.9 displays the limits for only the photon energy integration while the angular integration limits are left implicit. One should recall that the IR divergence arises from the amplitude contribution to the second term in Eqn. 4.9 in the limit $E_{\gamma} \rightarrow 0$. The IR divergence is present for any finite $\Delta E$ and must be dealt with. The extraction of useful physics comes most straightforwardly from the meson production process with no extra radiations. This process is embodied in the first term of Eqn. 4.9 and the Feynman diagram that goes along with such a process is pictured in Fig. 4.4 with the dashed line representing the outgoing pion. This first order contribution was parameterized in Chapter 1. Here the interest is

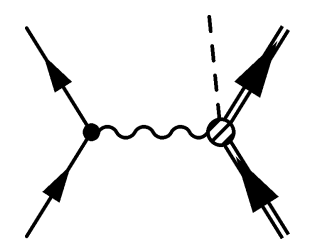

\section{Figure 4.4: Leading order meson production.}

in correcting the $\sigma_{m}^{m e s}$ cross section such that it is represented by the lowest order diagram contribution. A method for doing this has been developed by Afanasev et al. [2]. As one would expect, this calculation is model dependent and a MAID model is used in this work for the neutral pion production portion of the relevant diagrams [31]. The method of Afanasev calculates exactly the contributions from the pure QED portion of the matrix elements up to uncertainty in the hadronic models. The hadronic models, however, are included in a modular way such that better models (perhaps constrained by a first iteration of data analysis) can be included. The reference does not calculate radiations due to the hadronic currents and states that these are smaller by an order of magnitude and contain considerable theoretical uncertainties. This situation is understandable given the fact that the hadronic observables are typically poorly known in any new region of kinematics, 
and sparsely known in general. To calculate the photon emission probabilities the diagrams in displayed in Fig. 4.5 are used.

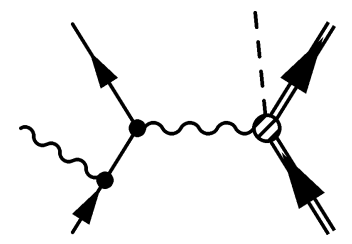

a)

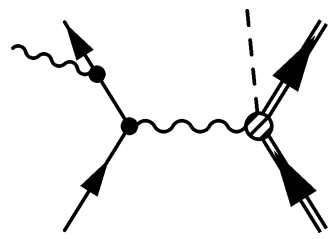

b)

Figure 4.5: $\mathcal{O}\left(\alpha^{\frac{3}{2}}\right)$ amplitude corrections for single meson production.

Once again, in order to cancel the IR divergences in the diagrams of Fig. 4.5 and restore the consistency of the perturbative expansion one must calculate the diagrams displayed in Fig. 4.6. In the approach of the mentioned reference the non-

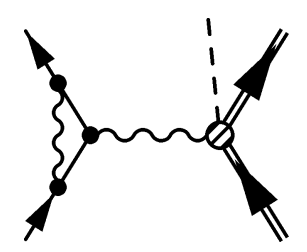

a)

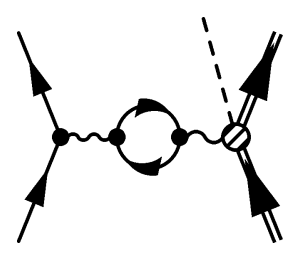

b)

Figure 4.6: $\mathcal{O}\left(\alpha^{2}\right)$ amplitude corrections for single meson production.

covariant approach of Ent et al. [36] is replaced by a covariant approach which uses instead of the maximum radiated energy, $\Delta E_{m}$, as a parameter uses the maximum value of the "inelasticity," $v$, which specifies the boundaries of missing mass to allow in the calculation and must be integrated over [11]. The inelasticity parameter is defined by:

$$
v \equiv\left(k_{\pi}+k_{\gamma}\right)^{2}-m_{\pi}^{2}
$$

for situations where the pions are undetected experimentally. The parameter is such that no radiation corresponds to the situation where $v=0$. If all particles were detected then the procedure would have the value of the inelasticity unambiguously specified with no need for integration. It is clear that the minimum value of $v$ is always zero due to the possibility of radiating a photon with arbitrarily low energy 
and the maximum value should correspond to the experimental data selection. Since in the current work it is advantageous to select pions via missing mass technique as will be discussed in Chapter 7, the method described here for radiative corrections is especially appropriate. Schematically the radiative correction can be written as in Eqn. 4.11 for the current situation.

$$
\sigma_{m}^{m e s}=\sigma^{m} e^{\delta_{i n f}}\left(1+\delta_{V R}+\delta_{v a c}\right)+\sigma_{F}
$$

The parameters $\delta_{i n f}, \delta_{V R}$ and $\delta_{v a c}$ parameterize the radiative correction in the same way as the elastic " $\delta$ " parameters did. Indeed the exponentiation of $\delta_{\text {inf }}$ to include low energy multi-photon effects is precisely analogous to the exponentiation of $\delta_{\text {soft }}\left(\Delta E_{m}\right)$ in the elastic radiative treatment. The term $\sigma_{F}$ is the IR divergence free part of the measured cross section utilizing the same separation of the IR divergent and IR divergence free portions of the cross section as the Afanasev reference ${ }^{5}$. The correction factor which must be applied to measured is defined by the number $\frac{1}{\delta}$ with:

$$
\delta \equiv \frac{\sigma_{m}^{m e s}}{\sigma^{m}}
$$

This correction factor must be applied to all the measured data in this work since the born cross section $\sigma^{m}$ is the one to be extracted.

\subsection{Incoherent Radiation and Straggling}

So far the background physical processes which have been treated in this chapter still pertain to scattering off of the main target proton. In other words one can say they are connected at the point of interaction to the detected hadron. Other interactions can, of course, occur as either of the exiting particles traverses experimental material including the target and target walls. Interactions which appreciably change the momentum of the outgoing particles are extremely rare because the signal scatterings are rare in and of themselves. Incoherent radiations and small angle scatters may, however, occur and only slightly modify the momentum of the

\footnotetext{
${ }^{5}$ It should be noted that this separation is somewhat arbitrary but any choice keeps the measured cross sections invariant.
} 
outgoing particles. These events are slightly less rare than other hard scattering events and so must be taken into account. The incoherent radiations are those which occur from the exiting lepton recoiling against target protons which were not involved in the initial hard scatter. These events have negligible probability for the exiting hadrons (protons). The incoherent radiative events do not involve the hard scattering vertex and can be calculated completely separately unlike the coherent effects which had a delicate interdependence on the signal processes.

There are many references on such events and the situation is very well understood theoretically. The corrections can be incorporated via Monte Carlo technique, and this is the action taken in this work.

\subsection{Multiple Scattering}

It can also happen that the outgoing protons for a scattering event are deflected within some collimator material through small angles and subsequently taken as data in the accepted range of kinematics. This effect is also well understood and taken into account via Monte Carlo technique. 


\section{CHAPTER 5 \\ Spectrometers and Electronics}

\subsection{Overview}

The pseudoscalar meson production experiment E01-002 took place in the Hall C of Thomas Jefferson National Accelerator Facility. While the main thrust of this work is to understand the physics and extract important measurements from the raw data of experiment E01-002, it is important to understand how that data was collected. This will give a context to all the physics which is discussed and also allow one to begin to think about systematic errors which will plague the observable extraction covered in Chapter 8. Systematic errors, unlike statistical (or counting) errors affect differing experimental setups in different (unrelated) ways. In order to quantify these errors which are apparatus dependent, one must know the measuring devices very well. Since Jefferson Lab is a facility which constantly maintains and upgrades its accelerator technology and practices in order to provide high quality electron beams to each of the experimental halls, it is clear that exposition of the beam creation and maintenance procedures is out of the scope of this work. The reader is referred to the accelerator group at Jefferson Lab for references on the beam preparation portion of the experimental setup. For an executive type overview of the machine capabilities, construction and design see [59]. Accelerator physics is an interesting field in its own right, there are many effects which must be overcome to deliver quality beam $[25,61]$. This work will begin from a energetically and spatially stable, high quality, continuous wave electron beam entering the experimental Hall C. With the exception of any mention of beam energy measurements or other beam diagnostics performed in the Hall C Arc, the experimental analysis presented here begins when the beam enters the hall.

\subsection{Beamline}

Prior to the interaction in the target the electron beam traverses several points of interest. These points correspond to the beam current monitoring, beam energy 
measurement and beam raster points. The experiment depends on knowing to a reasonable accuracy the beam energy, and current.

The energy of the electron beam is typically measured in a large arc bend just before the entrance to the experimental Hall C. Sensitive beam position detectors (harps) are used to monitor the position of the beam at two different locations of the bend. The information is then used to calculate the electron energy entering the hall. The energy uncertainty when using this technique is $5 \times 10^{-4} \mathrm{GeV}$. The current data from experiment E01-002 did not have the benefit of this type of arc measurement periodically throughout the run. Instead the beam energy was measured by this method at the beginning of the running period and used to calibrate a measurement of the energy using a bending dipole magnet prior to the hall and a series of beam position monitors which can be fit. This value has a similar error to the typical arc measurement and is assumed to have the same error. For the current experiment the energy varied somewhat during the first quarter of the run or so. The energy varied from approximately $E_{\text {beam }}=5.501 \mathrm{GeV}$ to as low as about $E_{\text {beam }}=5.492$ $\mathrm{GeV}$. After this period of variation the beam energy was stable and $E_{\text {beam }}=5.499$ $\mathrm{GeV}$. The small energy difference was taken into account in all simulation work and data reconstruction. Since results are not reported as a function of energy and the values of kinematics were calculated with the appropriate value for $E_{\text {beam }}$, the beam energy is stated to be $5.5 \mathrm{GeV}$ throughout this work when listing kinematics.

The beam current measurement is accomplished by using two beam current monitors (BCMs) positioned along the beam line. These current monitors are quite stable but do not have the ability to make an accurate absolute measurement. An additional current monitor, the Unser monitor, has what amounts to basically the opposite problem with a very accurate absolute current measurement which has an unstable zero [88], experiencing typical drifts of $3 \mu \mathrm{A}$. Luckily, it is an easy enough beam exercise to extract the Unser monitor zero since when the beam ramps down to zero the residual reading left on the monitor will be the offset. Therefore the best procedure to use (and the one used in this experiment) is to periodically calibrate the BCMs to the Unser monitor using a special run where the current is brought to zero and ramped up to several different currents. The error on the charge measurement 
was typically $1.0 \%$ for a run [8] and was stable throughout the E01-002 runs.

After several current monitors on the beam line there is a so-called "rastering" system. Electron beams tend to have very small spacial extent and therefore in cryogenic targets would induce significant boiling if the beam were allowed to impinge on the target for too long at a current of a few to several tens of micro Amps. For this reason, Hall C uses a system which sweeps the beam uniformly over a square pattern on the target. The size of this pattern is typically $\pm 1.2 \mathrm{~mm}$ in the $\mathrm{x}$ and $\mathrm{y}$ directions.

It should also be mentioned that the beam itself has a periodic time structure due to the RF techniques used to create and accelerate the beam. For the Jefferson Lab accelerator the frequency of this structure (corresponding to the excitation frequency of the cryogenic accelerator cavities) was $1497 \mathrm{MHz}$ which corresponds to beam pulses which are about 668 ps apart. The beam is delivered to each hall by a kicker which moves a third of the beam into each of the three hall beam pipes. Therefore when the beam arrives in each hall it will have bunches which are separated by roughly $2.0 \mathrm{~ns}$. This intrinsic beam structure will be important for subtracting coincidence spectrometer events which have two particles that do not correlate to the same beam bunch.

\section{$5.3 \quad$ Target}

After the beam line is understood it is useful to understand the target mechanism. The geometry of the target is especially important because of the possibility of electron scattering interactions in the target. These interactions will be quantified in Section 7.4.2 while the current section will deal with the logistics of the target carriage.

The target for the current experiment is a liquid Hydrogen " $\mathrm{LH}_{2}$ " target which was kept in a constant cooling loop with a temperature of $19.0 \mathrm{~K}$. The target ladder for the experiment contained several other targets along with a "dummy" target which was used for measuring the contribution to the data due to scattering in the target walls. The only targets used in the current experiment were the $\mathrm{LH}_{2}$ target and the Al dummy. The target cell was cylindrical and $4.013 \pm 0.008 \mathrm{~cm}$ in diameter, 
made of 7075 Aluminum ${ }^{1}$. The thickness of the target cell was measured at four places around the cylinder [66] and the results are reported in Tbl.5.1.

\begin{tabular}{|c|c|c|c|c|}
\hline Target Loop & top $(\mathrm{mm})$ & right $(\mathrm{mm})$ & bottom $(\mathrm{mm})$ & left $(\mathrm{mm})$ \\
\hline $\mathrm{LH}_{2}$ & $0.1384 \pm 0.0013$ & $0.1359 \pm 0.0013$ & $0.1270 \pm 0.0013$ & $0.1308 \pm 0.0013$ \\
\hline
\end{tabular}

Table 5.1: $\mathrm{LH}_{2}$ target wall thickness.

\subsection{Spectrometers}

Jefferson Lab Hall C houses two small acceptance but high resolution spectrometers. The spectrometers are called the High Momentum Spectrometer (HMS) and the Short Orbit Spectrometer (SOS). The naming of the latter is due to its ability to detect particles which have a shortened detectable flight path due to instability. The present section will outline the structure of these spectrometers as well as each of the components which are utilized for the exclusive analysis in experiment E01-002. The reader is referred to other references for description of unused components of the general HMS or SOS detector packages. For the experimental

\begin{tabular}{|c|c|}
\hline HMS detector & Utilized \\
\hline Drift Chamber 1 & $\sqrt{ }$ \\
\hline Drift Chamber 2 & $\sqrt{ }$ \\
\hline Front Hodoscope (X \& Y) & $\sqrt{ }$ \\
\hline Gas Cerenkov & \\
\hline Aerogel Cerenkov & $\sqrt{ }$ \\
\hline Rear Hodoscope (X \& Y) & $\sqrt{ }$ \\
\hline Calorimeter & \\
\hline
\end{tabular}

Table 5.2: HMS utilized detector components.

data in experiment E01-002, and for neutral pion production in Hall C in general, one measures a proton in the HMS spectrometer and an electron in the SOS spectrometer. Some of the detector components mentioned in Tbls. 5.2 and 5.3 are used for particle identification to accomplish the coincidence measurement. Though the coincidence data is the main focus, the spectrometers are set to read both coincidence

\footnotetext{
${ }^{1} 7075$ Aluminum is an Aluminum alloy which uses Zinc as the alloying agent.
} 


\begin{tabular}{|c|c|}
\hline SOS detector & Utilized \\
\hline Drift Chamber 1 & $\sqrt{ }$ \\
\hline Drift Chamber 2 & $\sqrt{ }$ \\
\hline Front Hodoscope (X \& Y) & $\sqrt{ }$ \\
\hline Gas Cerenkov & $\sqrt{ }$ \\
\hline Rear Hodoscope (X \& Y) & $\sqrt{ }$ \\
\hline Calorimeter & $\sqrt{ }$ \\
\hline
\end{tabular}

Table 5.3: SOS utilized detector components.

and single arm electron data using separate triggers. Here the interest is obviously single arm electron data using the SOS as a good cross check since below two pion threshold and aside from radiative corrections the inclusive data is only different from the exclusive data (either $\pi^{0}$ or $\pi^{+}$) by the reaction which is essentially just an isospin rotation ${ }^{2}$.

The spectrometers are situated in the hall such that the HMS is to the right when facing in the direction of the beam and the SOS is to the left. The range of angular (and momentum) settings used in the current experiment is reported in Section D.1 while the magnet settings to achieve the various momentum settings are reported in Section D.2. The layout of Hall C is pictured in Fig. 5.1. Aside from the detector packages of each of the spectrometers whose components were mentioned, there are some important general aspects of each of the spectrometers which should be considered.

The general parameters of the spectrometers including the magnetic structure will be discussed and each important component of the detector package will be visited in turn in this section. The first and most important thing to note about the spectrometers as far as accurate data acquisition is concerned is the coordinate systems that they use. The systems are largely determined by the logistics of the magnets used in the spectrometer as it is always desirable (from the perspective of magnet design and maintenance) to have the "z" axis moving down the central axis of the spectrometer and to have the " $\mathrm{x}$ " axis point in the positive dispersive direction. The "y" axis will then be defined by the choice of a right handed coor-

\footnotetext{
${ }^{2}$ Though, isospin is not conserved in electromagnetic interactions so this provides no hard constraint.
} 


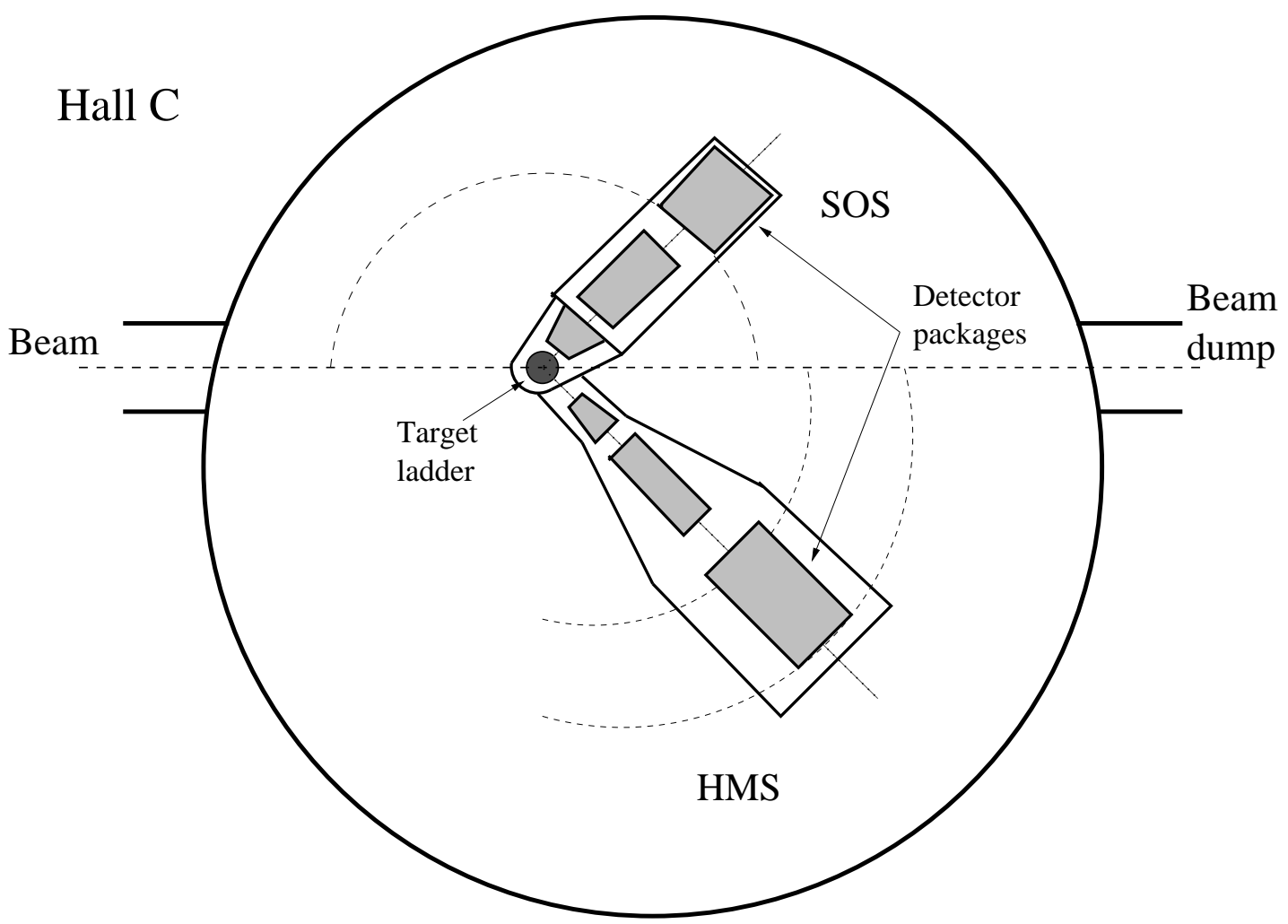

Figure 5.1: Top view of Hall C. The graphic was inspired by the publication of Armstrong [5, 6] and is not to scale.

dinate system. Figure 5.2 shows the coordinate systems of both the SOS and HMS spectrometers. Both the focal plane and target quantities will use this coordinate system for detected particles, in particular the change in the $\mathrm{x}$ or $\mathrm{y}$ coordinates per unit change in the $\mathrm{z}$ coordinate will be used to calculate angles. One should also note that the entrance to the spectrometers is accompanied by a collimator with different dimensions for the HMS and the SOS. The dimensions of the collimators are displayed in Fig. 5.3. It is also important to note that the flight distance from the target to the collimator is $166.37 \mathrm{~cm}$ for the HMS and $126.3 \mathrm{~cm}$ for the SOS spectrometer. Each of the collimators are $6.3 \mathrm{~cm}$ thick and with the exit openings being slightly larger than the entrance. The dimensions of Fig. 5.3 are not complete unless the apparent symmetry of the collimators is assumed.

The HMS and SOS use momentum dispersion due to dipole magnetic fields in order to analyze the momentum of particles. Different momenta will be displayed at 


\section{HMS}

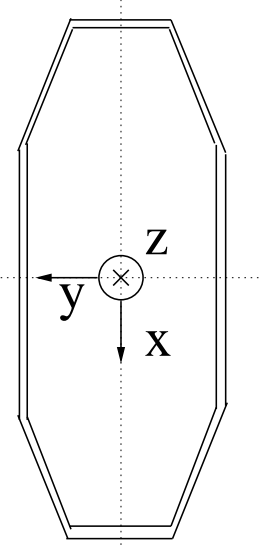

SOS

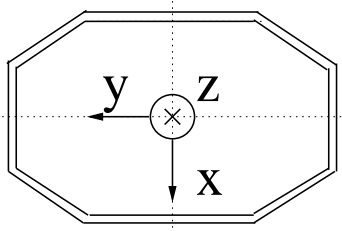

Figure 5.2: Spectrometer coordinate systems.

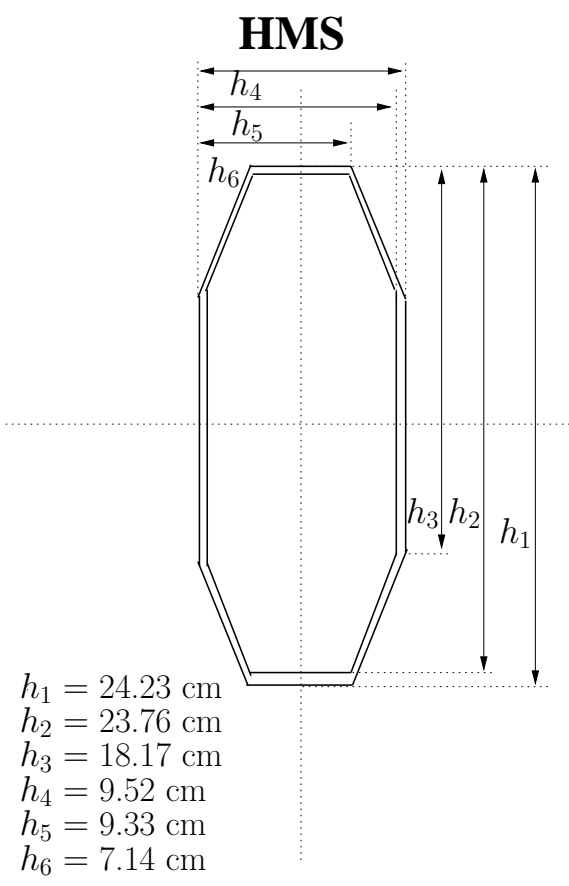

\section{SOS}

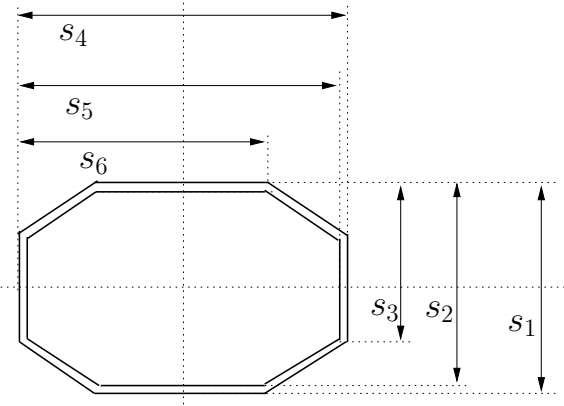

$$
\begin{aligned}
& s_{1}=9.87 \mathrm{~cm} \\
& s_{2}=9.63 \mathrm{~cm} \\
& s_{3}=6.32 \mathrm{~cm} \\
& s_{4}=15.14 \mathrm{~cm} \\
& s_{5}=14.77 \mathrm{~cm} \\
& s_{6}=10.99 \mathrm{~cm}
\end{aligned}
$$

Figure 5.3: Collimator dimensions. 
different positions inside the detector hut on a two dimensional surface referred to as the focal plane. In older magnetic spectrometers this two dimensional surface was actually made of a detection material which gave position (and thereby momentum) resolution. In modern times this plane is often not an actual physical entity but just a surface which one projects all the drift chamber data (or other particle trajectory data) back to. The modern specification of a focal plane can then be changed to minimize errors on the measured parameters. Each spectrometer in Hall $\mathrm{C}$ has its magnets configured to focus back to such a "virtual" focal plane. Figure 5.4 displays a schematic view of each spectrometer along with the magnet configurations $(\mathrm{Q}$ for quadrupole, D for dipole) and the location of the focal plane. The magnet

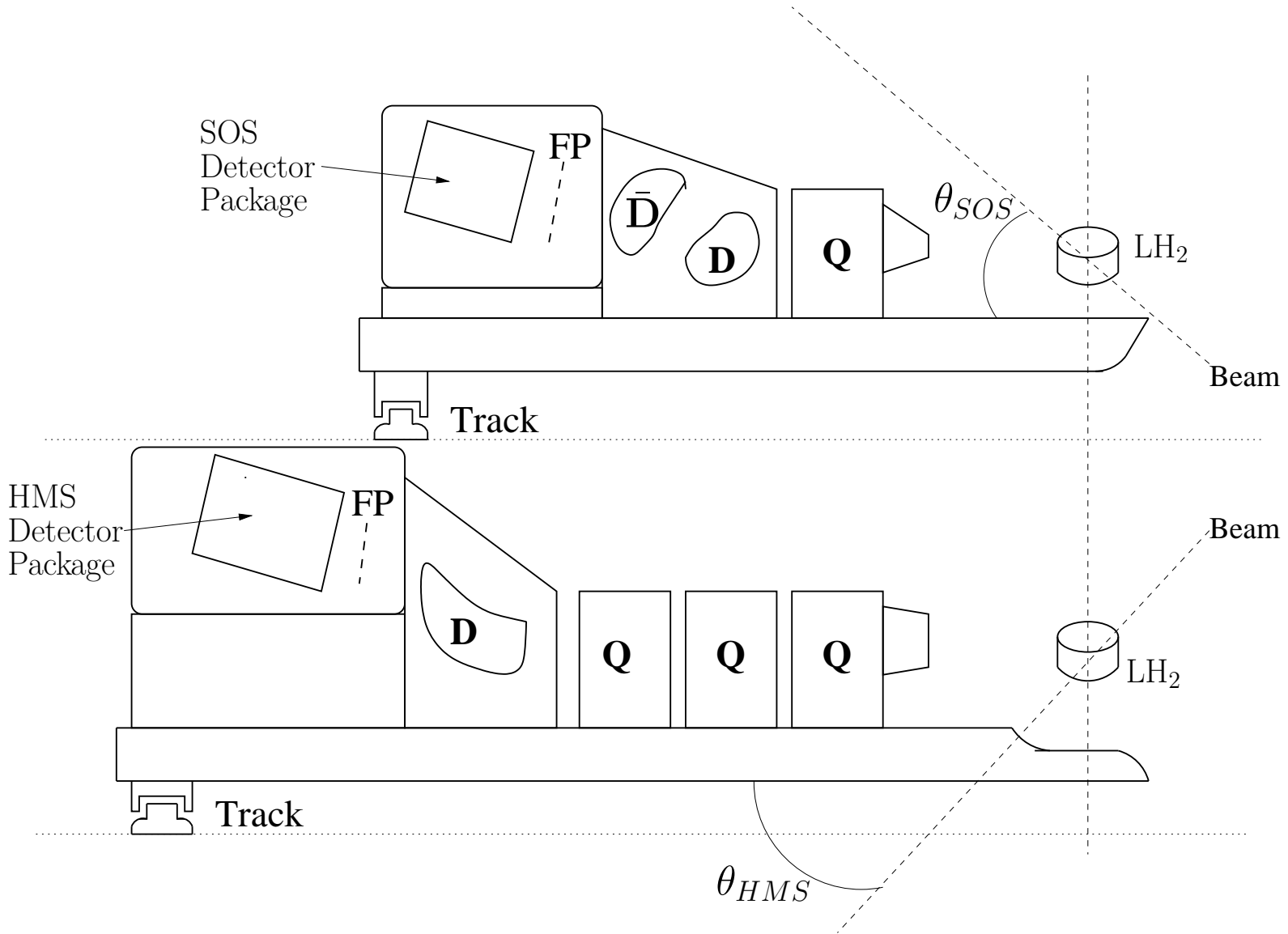

Figure 5.4: Schematic view of each spectrometer. The figure is not to scale. The label "Q" is for quadrupole, "D" is for dipole and "FP" represents the focal plane. 


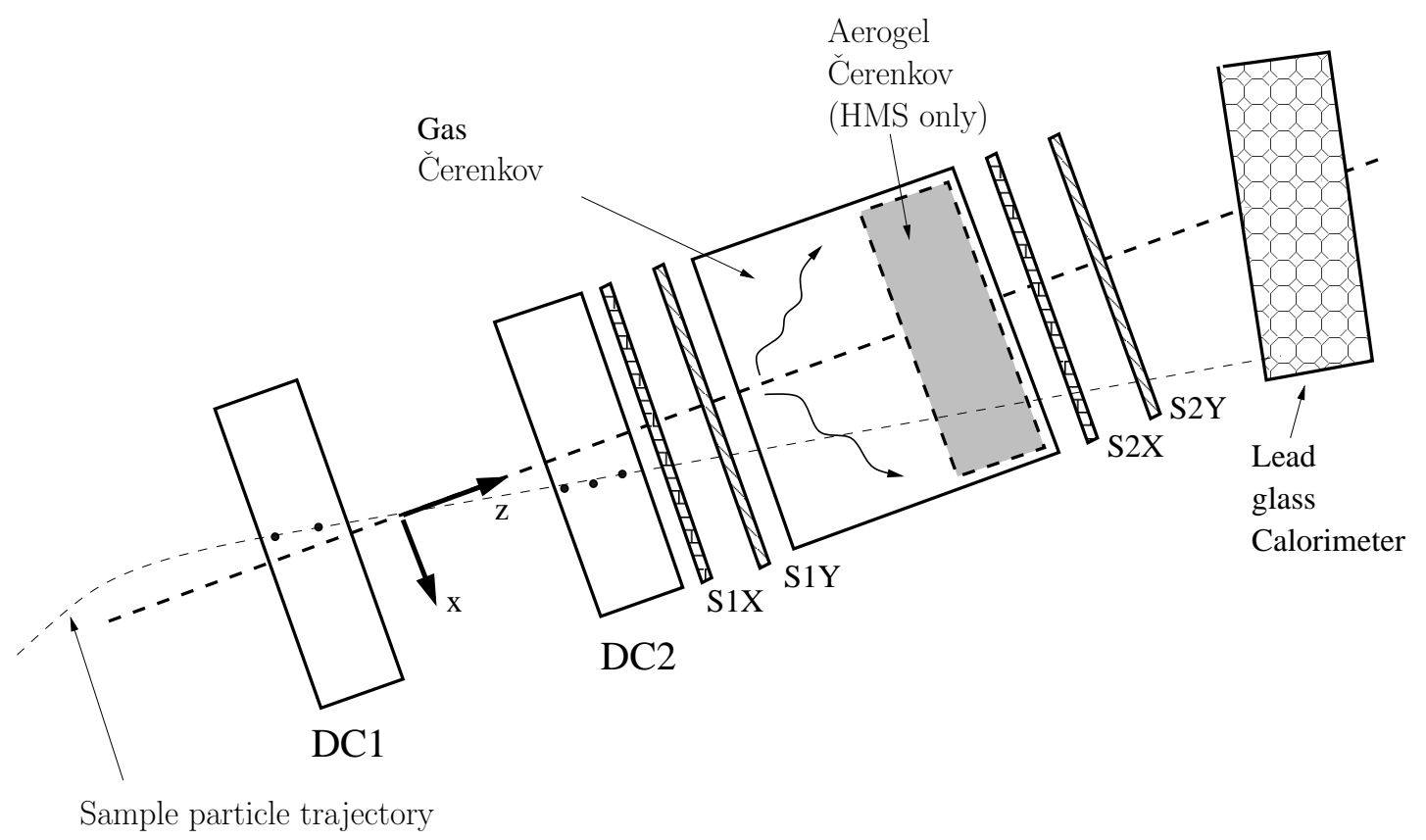

Figure 5.5: Typical spectrometer detector package. The graphic was inspired by the publication of Armstrong [5, 6]. A sample particle trajectory is shown with drift chamber hits.

configuration for the HMS is $Q Q Q D$ (three quadrupoles then a dipole) and the configuration for the SOS spectrometer is $Q D \bar{D}$, where the bar denotes a dipole of opposite polarity. The quadrupoles are used as focusing elements in general to allow the apparatus to accept events which would hit the spectrometer material had they not been focused prior to bending [46]. Both the HMS and SOS spectrometers use a point-to-point magnetic configuration, wherein particles which originate from a common point with common momenta will be focused to the same point on the focal plane. The magnets in the spectrometer are typically modeled by transport matrices in phase space where the matrix elements are fitted to data or obtained from a precise field map. Procedures for the optimization of the matrix elements for the magnets in Hall $\mathrm{C}$ have been refined over the years [33]. It should be mentioned that the SOS dipole saturates above about $1 \mathrm{GeV}$ in momentum so that a separate optic matrix had to be used for the $1.74 \mathrm{GeV}$ (low $Q^{2}$ ) setting in the current experiment. The HMS had the same magnet matrix for all settings. This fact leads to a somewhat poorer knowledge of the SOS acceptance than the HMS 
acceptance which will be discussed in Section 9.4.

The drift chambers are located on either side of the focal plane in each spectrometer (this is almost by definition) and the rest of the detector package is located after the last drift chamber. Figure 5.5 shows the typical detector package which is utilized in each spectrometer hut. One of the spectrometers has a detector (the aerogel Cerenkov detector) which is not pictured but is in line with the gas Čerenkov detector. The figure labels the drift chambers by "DC1" and "DC2" and the focal plane sits between them (but not exactly normal to the axis of the spectrometer). The two sets of X-Y hodoscopes are pictured on either side of the gas Cerenkov (and labeled S1X, S1Y, S2X and S2Y in order along $+\hat{\mathbf{z}}$ ), these are used for the electronics trigger in a 3-out-of-4 configuration, that is, a pretrigger is generated if 3 out of 4 of the hodoscope planes fire.

\subsection{Electronics and Data Acquisition}

The detectors produce only raw electrical (usually NIM-type) signals. These need to be interpreted and eventually read to a computer via $\mathrm{ADC}$ and $\mathrm{TCD}^{3}$ modules. However, for experiments which have continuous beams and high rates (on order of $\mathrm{MHz}$ ) there should be some selection mechanisms built into the electronics (which are fast) that prevent the computers (which are slow) from having to read every signal and inevitably missing a high portion of the physical events (or having large "deadtime," which is discussed in Section 6.6).

The basic electronics selection mechanisms and read out scheme is represented in Fig. 5.6. The four hodoscope planes on each detector are used to create a pretrigger. Since the hodoscopes are simply scintillator bars read out on either the $\mathrm{X}$ or $\mathrm{Y}$ edges, one can easily form a signal which will give an electronic logical true if any of these bars fires. If three out of four of the planes fire, a trigger signal is generated for each spectrometer. As Fig. 5.6 indicates, these pretriggers are then passed to a programmable module which decides which kind(s) of data acquisition triggers to produce. The so-called "8LM" programmable module will not produce a data

\footnotetext{
${ }^{3}$ The abbreviations ADC and TDC stand for analog-to-digital and time-to-digital conversion respectively.
} 


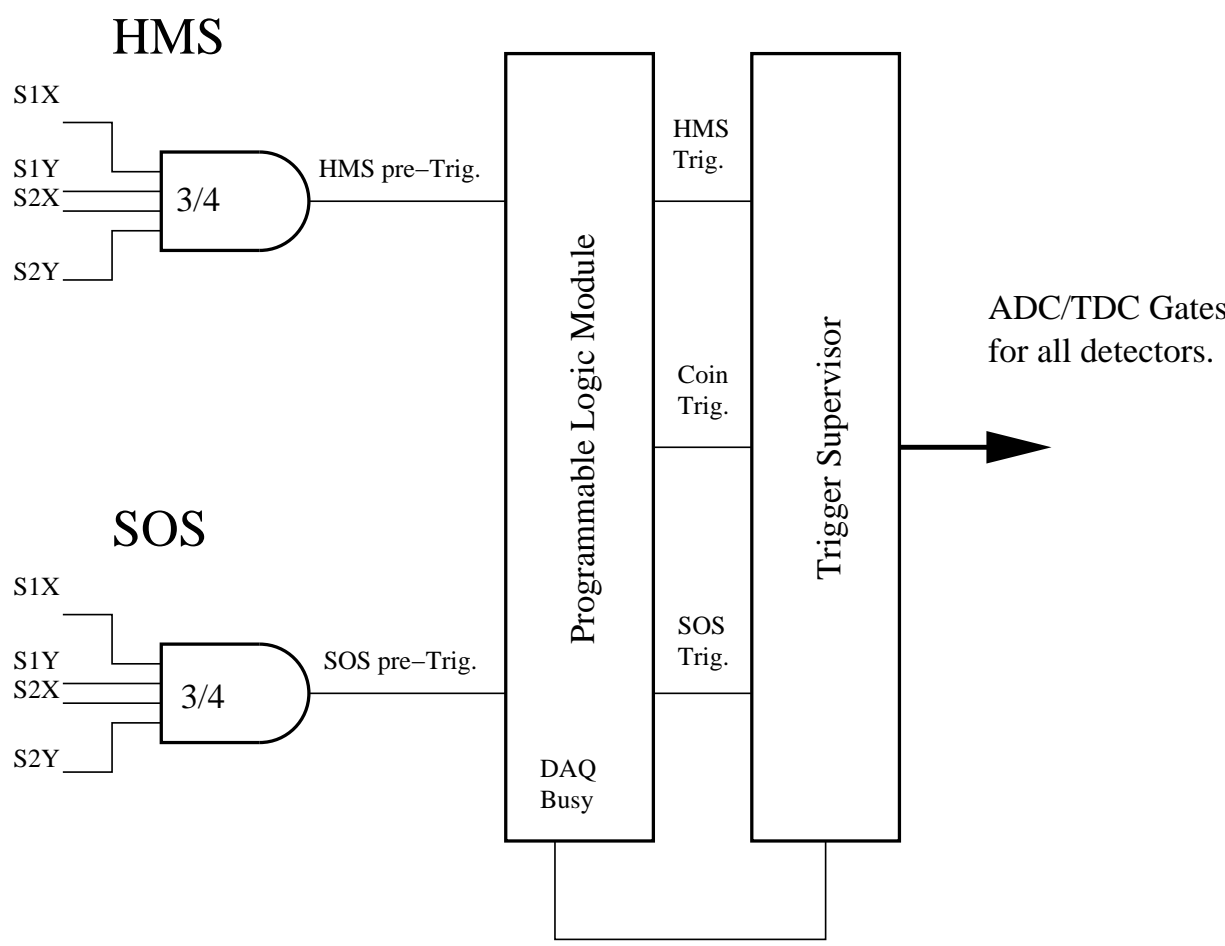

\section{Figure 5.6: Simple representation of the electronics and data acquisition system for experiment E01-002.}

acquisition (hereafter, DAQ) trigger if it receives a "busy" signal from the DAQ, indicating that the DAQ is not ready for another event yet. If the DAQ is not busy then the 8LM module will produce HMS, SOS, or coincidence triggers which are passed along to the trigger supervisor. The HMS and SOS triggers depend on only those detectors to fire, whereas the coincidence requires there to be a $\frac{3}{4}$ scintillator plane event in each detector. The timing between the SOS and HMS pretriggers was adjusted so that there was an overlap and therefore a possibility for a coincidence trigger. The appropriate signals finally arrive at the trigger supervisor, whose role is to control the DAQ by dispensing gates to the ADC and TDC modules only when a valid event is present and the DAQ is not already busy digitizing a previous event. The trigger supervisor also performs any necessary prescaling of the signals for example SOS singles often have prescale of a few to five ${ }^{4}$ and the HMS singles have prescales which are anywhere from 100 or so to a few thousand at the

\footnotetext{
${ }^{4}$ The SOS singles prescale factors used in this work are 1, 2, 3 and 5.
} 
low hadron momentum settings (where $\pi^{+}$production is copious). The prescaling allows the DAQ to skip some set number of events, or, read only every $n^{\text {th }}$ event where $n$ is the prescale factor. After the appropriate gates are dispensed for the appropriate triggers, the ADC and TDC modules (located in FastBus crates) will begin to digitize all relevant information concerning analog photomultiplier signals and time difference signals.

After the gates are sent out the computer DAQ system must perform its function. Hall $\mathrm{C}$ used the resident intranet connection to communicate with the CPUs which were carrying out the ADC or TDC conversions. The CODA [1] event builder is used at Jefferson Lab to retrieve all relevant information from the ADC and TDC modules while storing event information on disk and/or on tape. 


\section{CHAPTER 6 \\ Calibration and Efficiency}

\subsection{Absolute Measurements}

In counting experiments (which encompass most of the direct observables obtained from particle physics) it is not terribly difficult to obtain precise measurements. The precision of counting experiments is usually mostly a function of the

total time the experiment takes, since the statistical error goes like $\frac{\sqrt{n}}{n}$. Accuracy in counting experiments is quite a different story. It is typical for the accuracy of a measurement of the type considered in this work to depend strongly on the apparatus used and other analysis details. Usually the inaccuracy introduced via the analysis details are difficult to quantify straight away. This subject is left to Chapters 7 and 10 in the discussion of systematic errors. The present chapter concerns itself with inaccuracies which are apparatus dependent and somewhat readily quantifiable. In this case, once the processes or devices are understood corrections can be made to combat them and/or expected errors on the final counts can be quantified.

\subsection{Calorimeters}

The experiment utilized the SOS calorimeter to get a measurement of the energy deposited per unit momentum. Electrons will, of course, deposit the most energy since they are very susceptible to radiative events in the lead glass of the calorimeter (a high Z material). The value of $\frac{E}{p}$ should be near unity for electrons. This was ensured on a run-by-run basis in the post analysis. The calorimeter was calibrated so that the electron peak occurred very near unity (within 2.5\%). The widths of the electron peaks were stable within a percent. This fact allowed one to be very nearly certain that an electron was identified if $\frac{E}{p} \geq 0.8$. With the addition of the Čerenkov restriction and coincidence missing mass restrictions discussed in Section 7.2 , one is sure that electrons are identified very efficiently. 


\subsection{Drift Chambers}

Using the drift chambers to constrain the paths of good detector hits is essential to constraining the positions of particles at the spectrometer focal plane which is in turn used to fully reconstruct an event. Unfortunately, the process of using the drift chamber sense wire signals to reconstruct a particle path is not something that can be done with perfect efficiency or at any arbitrary detector rate. It is necessary to have a measure of how many good tracks are constructed compared to an estimate of how many good events probably traversed the drift regions. In the current experiment this was done by using the scintillator detectors to find all possible "good" events and then comparing this value to how many of these good events ended up with useable reconstructed tracks [53]. A good proton event was considered one with a number of photons in the HMS gas Čerenkov $\left(N_{h}^{\gamma}\right)$ below 0.5 and one with a number of photons in the HMS aerogel $\left(N_{a}^{\gamma}\right)$ to be below 2.0. A good electron event was considered one with calorimeter $\frac{E}{p}$ of greater than 0.7 while having a number of photons in the SOS gas Cerenkov of greater than 1.0. The tracking efficiency in each spectrometer was then monitored on a run by run basis. The HMS tracking efficiency averaged $95.2 \%$ while the SOS tracking efficiency averaged $99.5 \%$. Since the runs have a fixed rate at a given beam current and the beam currents were typically stable during a run (within reasonable limits), the average tracking efficiency per run was used to correct the data of that particular run.

\subsection{Target Boiling}

Although the beam rastering system was designed to distribute the heat load more evenly on the front face of the target, there can still be localized boiling. The best place to check for such an effect is in the SOS spectrometer rates, since there is only one configuration change of this spectrometer. If one observes a drop in the electron detection rate, this signifies a drop in the average density of the target and is most likely due to localized boiling. This effect should only be significant at the highest beam currents and can be added as a systematic error or corrected for in the high current runs. Figure 6.1 shows the count rates in the electron spectrometer as a function of beam current. The reported rates and currents correspond to the 
averages for each individual experimental run. It is clear that three different SOS prescale factors were used during the experiment. The SOS prescale factor, $f_{\text {sos }}$, is

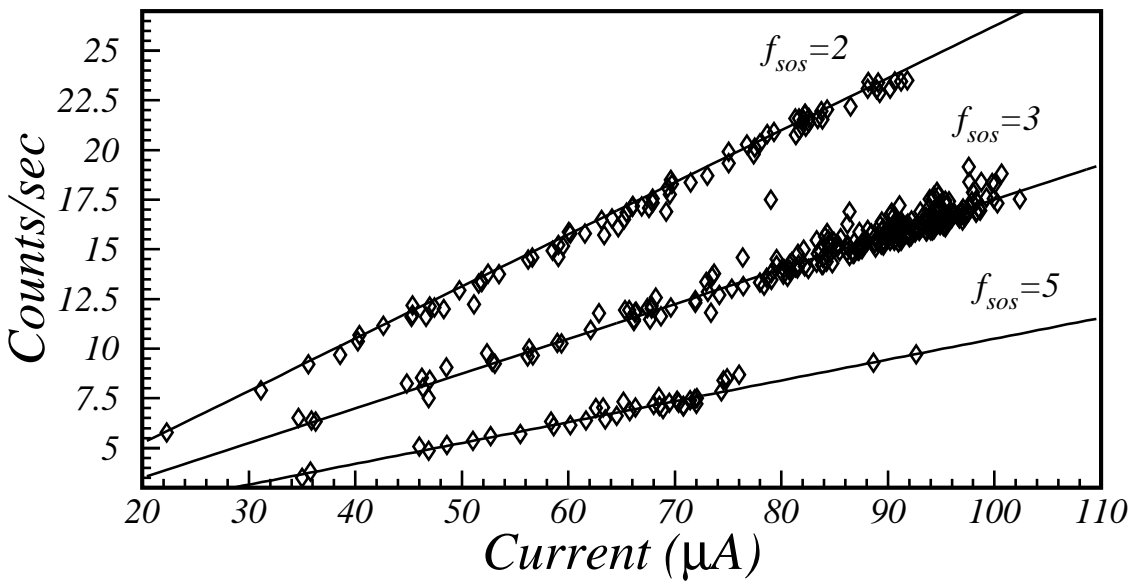

Figure 6.1: SOS single arm rates vs. current. $\theta_{\text {sos }}=47.5^{\circ}$ and $p_{\text {sos }}=1.74$ GeV.

reported with the figure and one can see that the average rates fall nicely on the expected lines with few exceptions. By scaling out the obvious linear dependence in these plots one can gain an idea about the possibility of target boiling. Figure 6.2

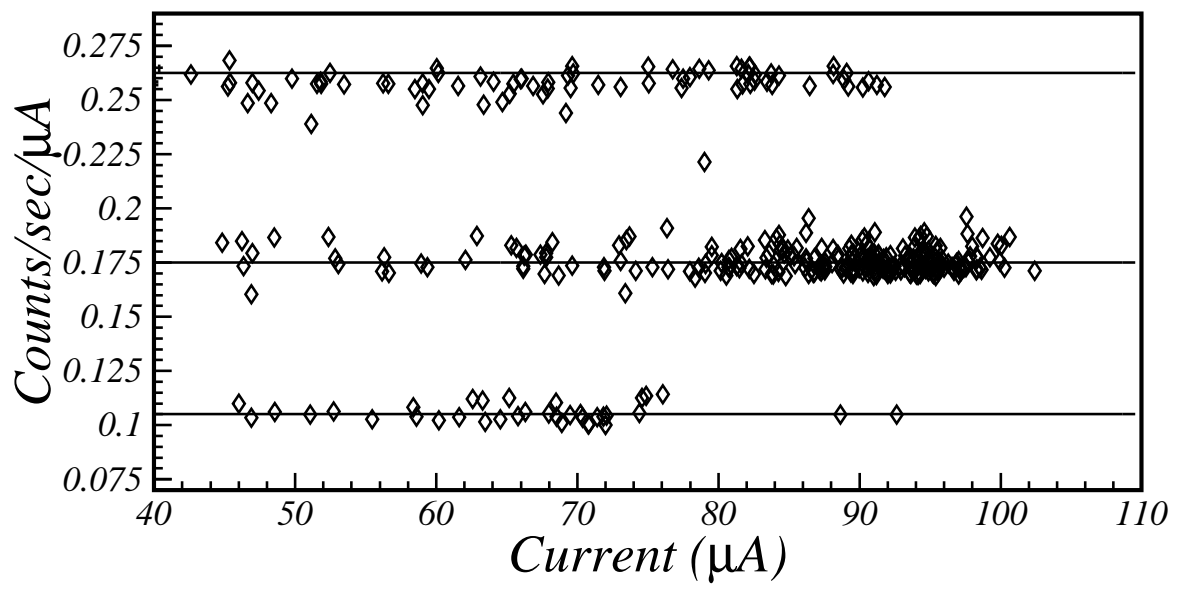

Figure 6.2: SOS single arm scaled rates vs. current. $\theta_{\text {sos }}=47.5^{\circ}$ and $p_{\text {sos }}=1.74 \mathrm{GeV}$.

shows that to a very good approximation the SOS singles rate increase per micro amp is constant. The especially encouraging part of this plot is that the scaled 
rate seems not to fall off on average, even at the highest utilized currents. This fact means that on average there is only a negligible amount of target boiling and therefore negligible rate fluctuations. To be precise and bound the error for the target boiling one can simply average the above data for each prescale factor from a minimum current up to the max. Doing this one produces Tbl. 6.1. In Tbl. 6.1 $\bar{r}_{i}$

\begin{tabular}{|c|c|c|c|c|c|c|}
\hline Min. Current $(\mu \mathrm{A})$ & $\bar{r}_{5}$ & $\sigma_{5}$ & $\bar{r}_{3}$ & $\sigma_{3}$ & $\bar{r}_{2}$ & $\sigma_{2}$ \\
\hline 20.0 & 0.1055 & 0.0039 & 0.1759 & 0.0056 & 0.2577 & 0.0065 \\
\hline 40.0 & 0.1056 & 0.0039 & 0.1759 & 0.0055 & 0.2578 & 0.0066 \\
\hline 60.0 & 0.1056 & 0.0042 & 0.1758 & 0.0054 & 0.2584 & 0.0068 \\
\hline 80.0 & 0.1048 & - & 0.1757 & 0.0053 & 0.2601 & 0.003435 \\
\hline 90.0 & - & - & 0.1757 & 0.0052 & 0.2570 & 0.001104 \\
\hline 95.0 & - & - & 0.1756 & 0.0056 & - & - \\
\hline
\end{tabular}

Table 6.1: Target rate statistics. $\theta_{\text {sos }}=47.5^{\circ}$ and $p_{\text {sos }}=1.74 \mathrm{GeV}$.

stands for the average reduced rate (rate per micro amp) for prescale factor "i," and $\sigma_{i}$ stands for the corresponding standard deviation. The table shows that there is no significant change in the average reduced rates. If one wanted to place very generous limits on the percentage error due to target boiling one can take the largest average negative deviation from the table and assume it is all due to target boiling. The only place were one sees a steady decrease in average reduced rate is for prescale factor 3. This is also the prescale factor group which probes the highest currents. Ignoring the fact that the deviation of -0.0003 over the current scan is well within the calculated errors, one obtains a bound for the target boiling of $0.17 \%$ on average. It is unnecessary to correct for such a small error but it will be noted. It should be emphasized that this calculation included only average boiling over runs and its result means that there is no significant deviation on the average sense. However, the boiling rates and deviations can be larger for small time slices within a particular run. This type of short timescale boiling will be washed out by statistical errors and is not considered in detail.

This analysis should also be carried out for the SOS spectrometer setting corresponding to the higher average $Q^{2}$. Figures 6.3 and 6.4 show the SOS spectrometer rates and reduced rates for the larger angle SOS setting. The figures show that 


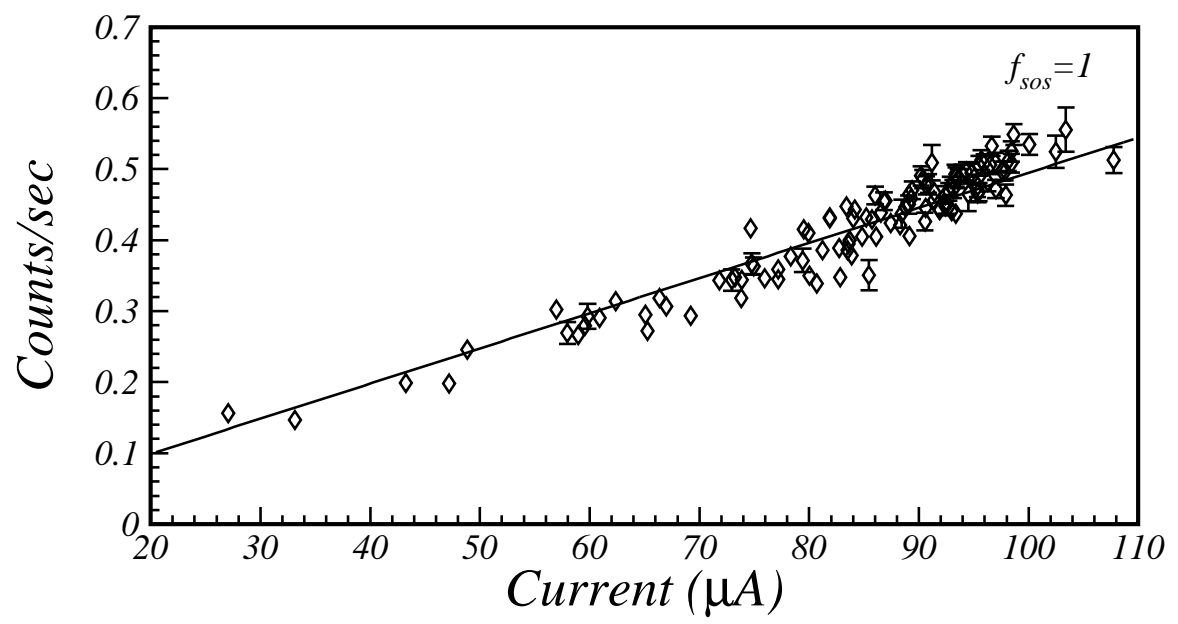

Figure 6.3: SOS single arm rates vs. current. $\theta_{\text {sos }}=69.9^{\circ}$ and $p_{\text {sos }}=1.04$ GeV.

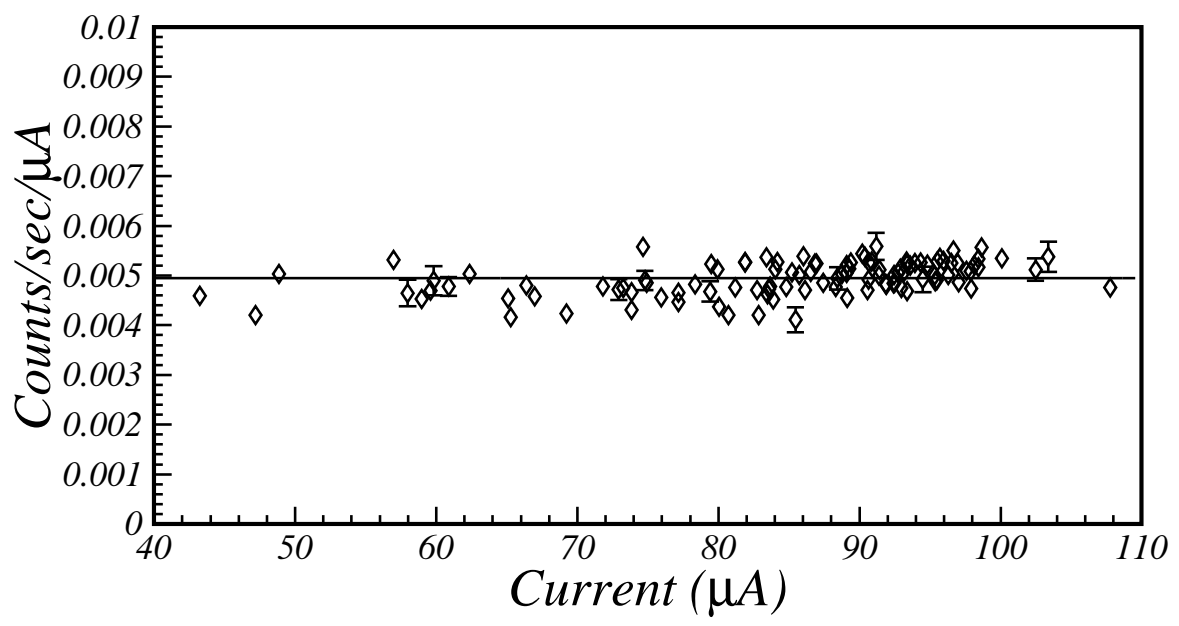

Figure 6.4: SOS single arm scaled rates vs. current. $\theta_{s o s}=69.9^{\circ}$ and $p_{\text {sos }}=1.04 \mathrm{GeV}$.

target boiling is a very small effect on average for the larger angle SOS setting as well. Since cross sections and therefore the rates fall quite rapidly as one goes to larger average $Q^{2}$, this SOS setting had a prescale factor of unity. The Tbl. 6.2 shows that there is no clear downward trend so that one can consider the target boiling for this SOS spectrometer setting as negligible, bounded by the previously quoted value.

Since on average the target boiling effect is basically undetectable, the conser- 


\begin{tabular}{|c|c|c|}
\hline Min. Current $(\mu \mathrm{A})$ & $\bar{r}_{1}$ & $\sigma_{1}$ \\
\hline 20.0 & $4.944 \times 10^{-3}$ & $3.480 \times 10^{-4}$ \\
\hline 40.0 & $4.941 \times 10^{-3}$ & $3.383 \times 10^{-4}$ \\
\hline 60.0 & $4.957 \times 10^{-3}$ & $3.347 \times 10^{-4}$ \\
\hline 80.0 & $5.025 \times 10^{-3}$ & $3.058 \times 10^{-4}$ \\
\hline 90.0 & $5.114 \times 10^{-3}$ & $2.328 \times 10^{-4}$ \\
\hline 95.0 & $5.154 \times 10^{-3}$ & $2.256 \times 10^{-4}$ \\
\hline
\end{tabular}

Table 6.2: Target rate statistics. $\theta_{\text {sos }}=69.9^{\circ}$ and $p_{\text {sos }}=1.04 \mathrm{GeV}$.

vative estimate of $0.5 \%$ will be used to account for the target boiling effect on the systematic error.

\subsection{Proton Absorption}

Because the proton interacts strongly there is a reasonable probability that it will interact with the nuclei in either the target material or the material that makes up the HMS detection package. This means that the HMS will have an inefficiency and this effect is termed "proton absorption."

Unfortunately the present experiment did not have dedicated elastic runs within the running period. However an estimate to the inefficiency that this effect caused can be made and checked with data from an experiment which ran just after the exclusive pion data taking at Jefferson Lab Hall C. The physics governing the proton absorption is obviously the nuclear proton-nucleon interaction. The measurement of this effect events shows that for proton-proton interactions, one has a cross section varying from about 47 to $42 \mathrm{mb}$ over the range of incident proton momentum from 2 to $5 \mathrm{GeV}$ [93]. Thus, in the current experiment the cross section for this interaction can be taken as constant for all settings and equal to the average value over this range with little error. For heavier nuclei the cross section can be approximated as $\sigma_{p p} A^{0.7}$, where $\sigma_{p p}$ is the previously quoted proton-proton cross section. The main interaction points in the HMS spectrometer are the scintillator planes $(1.0 \mathrm{~cm}$ thick) and aluminum windows of the gas Čerenkov and aerogel detectors which have total thickness of $0.51 \mathrm{~cm}$. Using the measured cross sections to compute the proton disappearance one obtains that $95 \%$ of the protons are detected 
by the HMS.

Since the kinematics of $e p \rightarrow e^{\prime} p^{\prime}$ events are fully constrained by a single arm measurement, this can be used to check the proton disappearance rate. For a configuration with $\theta_{S O S}=50.01^{\circ}$ and $\theta_{H M S}=18.00^{\circ}$ this process was measured [54]. The SOS and HMS central momenta were $1.74 \mathrm{GeV}$ and $4.335 \mathrm{GeV}$ respectively, while the beam energy was $5.247 \mathrm{GeV}$. The data acquisition records single arm events from both the SOS and HMS spectrometers in addition to coincidence events. Given this, one can compare the electron arm (SOS) elastic events to the coincidence elastic events in the pure elastic region of invariant energy, $0.9 \leq W \leq 1.0 \mathrm{GeV}$. In principle these should count precisely the same number of events if a central region of each detector is selected so that the acceptance is unity. Here the SOS in-plane angle was constrained to be $\pm 50 \mathrm{mr}$ which guaranteed the proton would arrive within the region of the HMS which has acceptance of unity. Further, the typical particle identification cuts for SOS electrons were applied as in Section 7.2 along with the time of flight restriction for the coincidence events displayed in Eqn. 7.3. The result of this study was 2451 coincident events and 254 more single arm events. The contribution from the target end caps for the single arm measurement was measured with a dummy target in this situation to be $127 \pm 12$. Similarly, the dummy target produced $11 \pm 3$ events. Subtracting these events and comparing the elastic yields one obtains that the proton absorption effect causes an inefficiency of approximately $4.0 \pm 1.0 \%$. That is, the coincidence case registered $95 \pm 1 \%$ of the single arm events. This measurement is in good agreement with the simple prediction and so will be used as an estimate for the proton absorption effect.

\subsection{Deadtime}

Even with extremely sensitive equipment and detectors with high resolution and very fast recovery properties it is impossible to take data at high rates (as are observed in this experiment) flawlessly. Between the response of detector materials and the saved computer data files there is some loss. In this experiment (and in general for the most part) there are two types of losses which one must deal with in the data acquisition. These are electronic and computer deadtime. Roughly, 
electronic deadtime refers to the inability of the electronics to "recover" from an event infinitely fast and the computer deadtime refers to the inability of the data reading system to "recover" infinitely quickly.

The electronic deadtime is mainly due to the fact that the gates created by the trigger supervisor (see Section 5.5) have finite width and therefore finite probability of encountering two events within a gate. If this happens the computer still makes only one read to record information and so not all of the relevant physical information for the two events is retained. A schematic of this gate timing situation is shown in Fig. 6.5. Fortunately, simple scaler counters can operate without any sophisticated

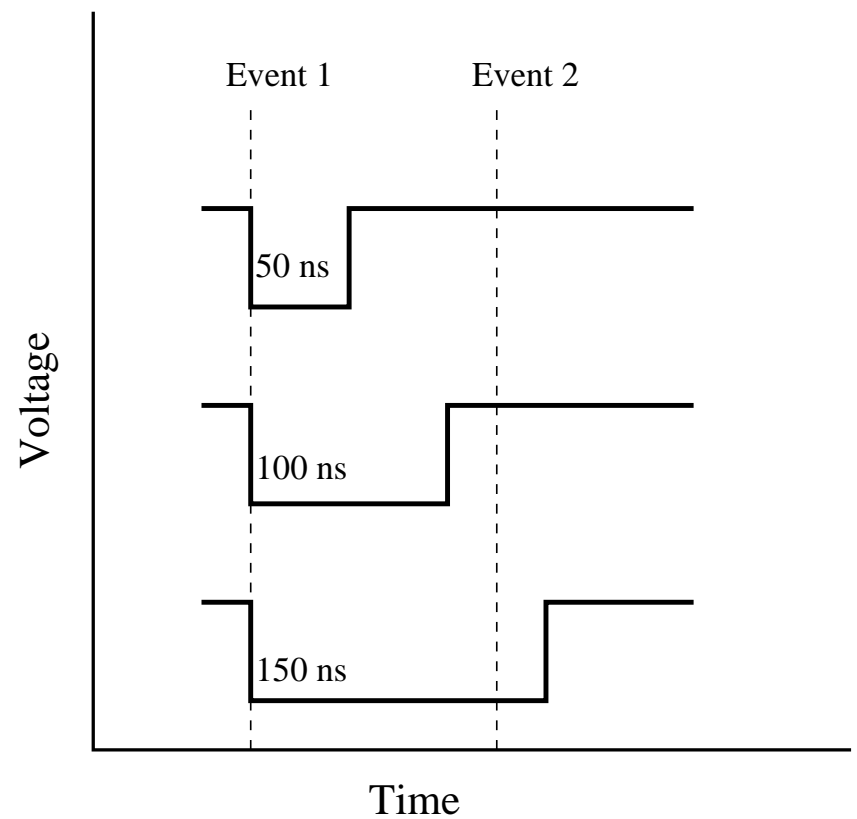

\section{Figure 6.5: Schematic of several gate signals and times of two random events. Depending on the gate width, they can arrive within the same electronic gate.}

gating to obtain information like rate and number of counts. This means that if one had a scaler read (that is, rate information) for many different gate sizes one could possibly find a correction which would extrapolate the final number of counts back to what would have been observed at zero gate width. This should remove the effect of finite gate size and thus correct for the electronic deadtime. This can essentially 
be done on a run by run basis ${ }^{1}$ using a setup displayed in Fig. 6.6. Using the fact

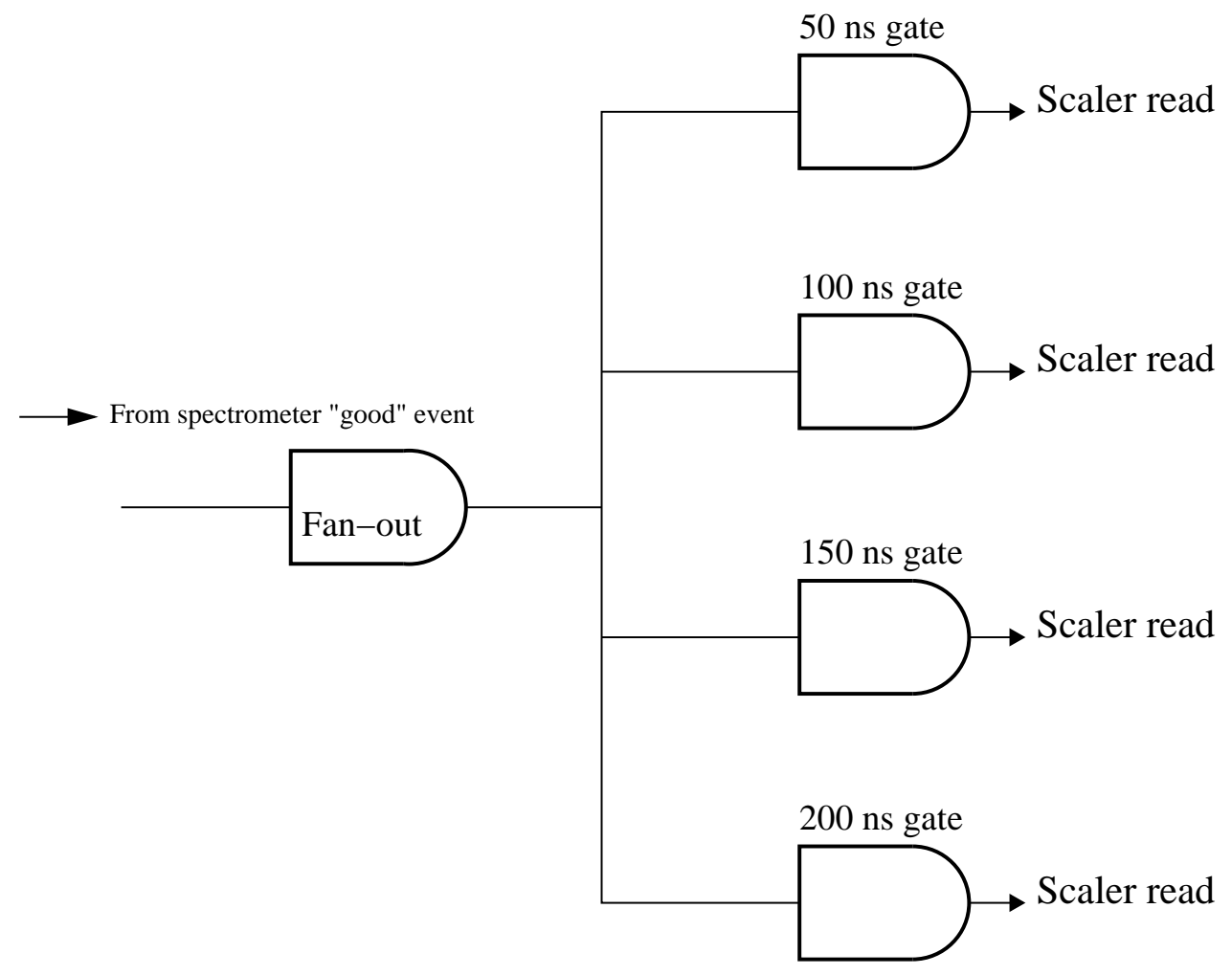

Figure 6.6: Schematic of the setup to measure the electronic deadtime.

that the probability for a count to arrive at a time $t$ greater than some other time $\tau$ is exponential, one can estimate the electronic dead time [43].

$$
P(t>\tau)=e^{-R \tau} \simeq 1-R \tau
$$

The symbol $R$ represents the detection rate. One knows that the approximation in equation 6.1 as long as one has a small dead time since in this equation $\tau$ would represent the gate width and we are examining the probability for not having a double count inside a gate which is near unity for small dead time. Any observed degradation of rate is due to the situation which is the converse of this probability, that is, the probability that a count will come at some time $t$ less than $\tau$. This probability is $R \tau$. Now, with the different gate widths described above, one knows

\footnotetext{
${ }^{1}$ The electronic dead time should be constant throughout a run since the spectrometer configuration (and therefore the rates) are not changing.
} 
that the difference in a number of observed counts for two different gate widths would be proportional to the difference of these probabilities.

$$
N_{100}-N_{200}=R(100 n s)
$$

With $N_{100}$ being the number of counts on the 100 ns gate in the previous figure and $N_{200}$ correspondingly for the $200 \mathrm{~ns}$ gate. Then one can use this equation to solve for the "true" number of counts. In words, this is the measured number of counts plus some factor times the nominal gate width.

$$
N_{\text {true }}=N_{\text {meas }}+R \tau_{\text {nom }}
$$

The factors $N_{\text {true }}$ and $N_{\text {meas }}$ are the true and measured number of counts. The factor $R$ can be obtained from the previous equation and used to obtain:

$$
\frac{N_{\text {meas }}}{N_{\text {true }}}=1-\frac{N_{100}-N_{200}}{N_{\text {true }}} \frac{\tau_{\text {nom }}}{100 n s}
$$

For calculational purposes one can substitute $N_{100}$ into the right hand side of Eqn. 6.4 for $N_{\text {true }}$ to get quick running estimate of the deadtime. Using this procedure the electric deadtime is $0.49 \%$ on average. This is small and thus justifies any other smallness assumptions in the calculations.

The computer dead time can be calculated simply by comparing the number of pretriggers to the number of triggers. Recalling Fig. 5.6, one can see that this comparison gives a direct measure of the average percentage of counts which observed the 8LM programmable logic module as busy. The computer dead time can then be quoted as $\frac{N_{\text {pre }}}{N_{\text {trig }}}$. The symbol $N_{\text {pre }}$ is the number of pretriggers whereas $N_{\text {trig }}$ is the number of triggers created by the $8 \mathrm{LM}$ module. The computer deadtime was estimated to be $6.8 \%$ on average for this experiment. 


\section{CHAPTER 7 Experimental Corrections}

\subsection{Realistic Measurements}

In a realistic world any investigator of natural science must deal with the fact that calculation prefers the simplest possible physical situation and often experiments can cooperate only approximately. In some cases it will become clear that even a "perfect" experiment will not do to isolate the simplest situation in our physical world. This fact is especially true in particle physics. In most cases the parameters which one is interested in are not related in a simple way to the experimental observables ${ }^{1}$ (traditionally cross sections). Indeed this fact should not be surprising since the entire goal of science is to explain all phenomenon from a few general (and therefore abstract) principles.

Because of the fact that experimental evidence cannot be perfectly matched to a theoretical calculation one can either modify the calculation to predict more realistic situations or modify the data to "extract" the simpler situation. Since it is advantageous to keep the theoretical calculations as simple as possible so as to look at only the basic parameters of the theory, one typically chooses to modify the experimental data. This is the method advocated in the current study. This data modification can be neatly summarized into three categories which are all very important for particle physics mostly due to the field theoretic nature of theoretical treatments of electromagnetic interactions.

1. Logistical Corrections: Corrections for which the current detector system has sufficient resolution to make reliably. In this case the detector will be used to reliably reject portions of data as not pertaining to the process of interest.

2. Empirical Corrections: Corrections for which the current detector system does not have sufficient resolution to make reliably. In this case empirical

\footnotetext{
${ }^{1}$ This fact is true in quantum field theory because of the arbitrariness of so called "bare" parameters.
} 
determinations of the contamination or theoretical models should be used to reject the uninteresting portions of the data set.

3. Physical Corrections: Corrections for processes which can never be separated from the processes of interest, even for "perfect" experiments ${ }^{2}$. These corrections are often a direct consequence of the nature of quantum mechanics and arise either because the processes simply cannot be distinguished or the theory used to predict the background processes necessarily involves the process of interest.

The present chapter will treat most such corrections for the meson production process. The examples of each of the above corrections are particle identification (PID) type rejections and elastic radiative corrections respectively. Dealing with the meson production radiative events will be relegated to Section 8.7.

\subsection{Data Exclusion}

Logistical corrections can include fiducial volume restrictions and restrictions which ensure proper particle identification. Each such correction is important to the success of an experiment but there are no serious problems with making precise rejections in most experiments including the current one. Therefore one need not make empirical modifications which attempt to account for detector resolution.

It might be useful to make a table of some variable names and symbols that will be used to describe them. A short description of the variables is also given below.

The data rejection can be divided into three different general categories which are fiducial, particle identification, and missing mass. The fiducial rejections are listed below with the exception of the collimator slit rejection which is described in the following paragraph. First one should define some variables for one of the (more complicated) exclusions.

\footnotetext{
${ }^{2}$ Experiments with no resolution effects.
} 


\begin{tabular}{|c|c|c|}
\hline Symbol & N-tuple Identifier & Description \\
\hline$y_{s}^{\prime}$ & ssyptar & SOS y angle target \\
\hline$x_{s}^{\prime}$ & ssxptar & SOS x angle target \\
\hline$y_{s}$ & ssytar & SOS y position target \\
\hline$X_{s}$ & ssxfp & SOS y position focal plane \\
\hline$Y_{s}$ & ssyfp & HMS y angle target \\
\hline$y_{h}^{\prime}$ & hsyptar & HMS x angle target \\
\hline$x_{h}^{\prime}$ & hsxptar & HMS x position focal plane \\
\hline$y_{h}$ & hsytar & HMS y position focal plane \\
\hline$X_{h}$ & hsxfp & SOS percent central momentum (\%) \\
\hline$Y_{h}$ & hsyfp & HMS percent central momentum $(\%)$ \\
\hline$\delta_{s}$ & ssdelta & SOS shower counter sum \\
\hline$\delta_{h}$ & hsdelta & SOS Cerenkov number of photons \\
\hline$t_{c}$ & cointime & Cosine of the hadronic angle in COM ${ }^{3}$ \\
\hline$\beta_{s}$ & ssshsum & Hadronic out of plane angle in COM \\
\hline$N_{s}^{\gamma}$ & scer_npe & Missing mass of hadronic system \\
\hline $\cos \theta^{*}$ & cos_thet & phicm \\
\hline$\phi^{*}$ & mmass2 & \\
\hline$M_{x}^{2}$ & &
\end{tabular}

Table 7.1: Variable Names.

$$
\begin{aligned}
& y_{\text {min }}^{\prime} \equiv \frac{1}{1000}\left(-125.0+4.25 \delta_{s}+64.0 y_{s}-1.7 \delta_{s} y_{s}\right) \\
& y_{\text {max }}^{\prime} \equiv \frac{1}{1000}\left(125.0-4.25 \delta_{s}+64.0 y_{s}-1.7 \delta_{s} y_{s}\right)
\end{aligned}
$$

With these definitions the fiducial exclusions are:

$$
\begin{aligned}
-20.0 & \leq X_{s} \leq 22.0 \\
y_{\min }^{\prime} & \leq y_{s}^{\prime} \leq y_{\max }^{\prime} \\
-15.0 & \leq \delta_{s} \leq 18.0 \\
-9.0 & \leq \delta_{h} \leq 9.0
\end{aligned}
$$

The collimator slit functions can be implemented by sampling the value of some function of a "tolerance" variable. This function will reproduce the octagonal geometry of the collimator but has the flexibility via the tolerance variable to accept some particles which traced back to inside the collimator. The SOS collimator function is represented by $f_{s}(t)$ and the HMS version is $f_{h}(t)$. The variable $t$ is the 
tolerance and $f_{s}(0), f_{h}(0)$ will represent boarders precisely at the geometric edges of the collimator. The definition of the function will be delayed to a later section. The restrictions placed on the data are as follows:

$$
\begin{aligned}
& f_{s}(0)=1 \\
& f_{h}(0)=1
\end{aligned}
$$

The particle identification exclusions can also be defined with the variables above. While the missing mass $\left(M_{x}^{2}\right)$ requirement is for particle identification, it need not be used in some analysis methods and thus will be considered separately. The particle identification cuts are listed in Eqn. 7.3 assuming that the coincidence time peak of interest is centered about $T$.

$$
\begin{aligned}
\left|t_{c}-T\right| & \leq 1.5 \\
\beta_{s} & \geq 0.8 \\
N_{s}^{\gamma} & \geq 0.5
\end{aligned}
$$

The missing mass requirement is made as a function of $\cos \theta^{*}$ to account for the resolution change in the double arm measurement.

$$
\begin{gathered}
M_{\text {min }}^{2}=\left(-a_{1} \cos \theta^{*}+a_{1}+a_{2}+a_{3}\right) \\
M_{\text {max }}^{2}=\left(a_{4} \cos \theta^{*}+a_{4}+a_{5}+a_{3}\right) \\
a_{1}=\frac{0.035}{2.0} \\
a_{2}=0.01 \\
a_{3}=0.28146 \\
a_{4}=\frac{0.045}{2.0} \\
a_{5}=0.03
\end{gathered}
$$

The missing mass requirement can then be expressed as the following:

$$
M_{\min }^{2} \leq M_{x}^{2} \leq M_{\max }^{2}
$$




\subsubsection{Collimator Functions}

The collimator functions $f_{s}$ and $f_{h}$ can be simply determined by the geometry and orientation of the collimator. The object of such a function is to return a value of unity if the particle traces back to an acceptable region at the collimator and zero otherwise. One can define a set of variables which represent the positions projected to the front and back face of the SOS and HMS collimator.

\begin{tabular}{|c|c|}
\hline Symbol & Description \\
\hline$\tilde{y}_{s}^{f}$ & SOS y front collimator face \\
\hline$\tilde{y}_{s}^{r}$ & SOS y rear collimator face \\
\hline$\tilde{x}_{s}^{f}$ & SOS x front collimator face \\
\hline$\tilde{x}_{s}^{r}$ & SOS x rear collimator face \\
\hline$\tilde{y}_{h}^{f}$ & HMS y front collimator face \\
\hline$\tilde{y}_{h}^{r}$ & HMS y rear collimator face \\
\hline$\tilde{x}_{h}^{f}$ & HMS x front collimator face \\
\hline$\tilde{x}_{h}^{r}$ & HMS x rear collimator face \\
\hline$h_{e}^{s}=7.201$ & SOS entrance collimator height \\
\hline$h_{e x}^{s}=7.567$ & SOS exit collimator height \\
\hline$v_{e}^{s}=4.696$ & SOS entrance collimator width \\
\hline$v_{e x}^{s}=4.935$ & SOS exit collimator width \\
\hline$h_{e}^{h}=4.575$ & HMS entrance collimator height \\
\hline$h_{e x}^{h}=4.579$ & HMS exit collimator height \\
\hline$v_{e}^{h}=11.646$ & HMS entrance collimator width \\
\hline$v_{e x}^{h}=12.114$ & HMS exit collimator width \\
\hline$x_{o}^{s}=0$ & SOS x collimator offset \\
\hline$y_{o}^{s}=0$ & SOS y collimator offset \\
\hline$x_{o}^{h}=0$ & HMS x collimator offset \\
\hline$y_{o}^{h}=0.028$ & HMS y collimator offset \\
\hline
\end{tabular}

Table 7.2: Projected positions.

\subsubsection{SOS Collimator}

The SOS collimator is set at a particular distance from the target and we can thus define the projected target positions for the SOS. 


$$
\begin{aligned}
& \tilde{y}_{s}^{f} \equiv y_{s}+126.3 y_{s}^{\prime} \\
& \tilde{x}_{s}^{f} \equiv 126.3 x_{s}^{\prime} \\
& \tilde{y}_{s}^{r} \equiv y_{s}+(126.3+6.3) y_{s}^{\prime} \\
& \tilde{x}_{s}^{r} \equiv(126.3+6.3) x_{s}^{\prime}
\end{aligned}
$$

If all of the following inequalities hold then the event is inside the collimator-plustolerance region and will be rejected, i.e. $f_{s}(t)=1$.

$$
\begin{aligned}
\left|\tilde{y}_{s}^{f}-y_{o}^{s}\right| & <\left(h_{e}^{s}+t\right) \\
\left|\tilde{x}_{s}^{f}-x_{o}^{s}\right| & <\left(v_{e}^{s}+t\right) \\
\left|\tilde{x}_{s}^{f}-x_{o}^{s}\right| & <\left(\frac{-v_{e}^{s}}{h_{e}^{s}}\left|\tilde{y}_{s}^{f}-y_{o}^{s}\right|+3.0 \frac{v_{e}^{s}}{2.0}+t\right) \\
\left|\tilde{y}_{s}^{r}-y_{o}^{s}\right| & <\left(h_{e x}^{s}+t\right) \\
\left|\tilde{x}_{s}^{r}-x_{o}^{s}\right| & <\left(v_{e x}^{s}+t\right) \\
\left|\tilde{x}_{s}^{r}-x_{o}^{s}\right| & <\left(\frac{-v_{e x}^{s}}{h_{e x}^{s}}\left|\tilde{y}_{s}^{f}-y_{o}^{s}\right|+3.0 \frac{v_{e x}^{s}}{2.0}+t\right)
\end{aligned}
$$

So to summarize $f_{s}(t)$ is piecewise and defined by the above condition for being "inside" the collimator-plus-tolerance.

$$
f_{s}(t)= \begin{cases}1, & \text { inside } \\ 0, & \text { outside }\end{cases}
$$

\subsubsection{HMS Collimator}

The HMS collimator is set at a particular distance from the target and we can thus define the projected target positions for the HMS.

$$
\begin{aligned}
\tilde{y}_{h}^{f} & \equiv y_{h}+126.2 y_{h}^{\prime} \\
\tilde{x}_{h}^{f} & \equiv 126.2 x_{h}^{\prime} \\
\tilde{y}_{h}^{r} & \equiv y_{h}+(126.2+6.3) y_{h}^{\prime} \\
\tilde{x}_{h}^{r} & \equiv(126.2+6.3) x_{h}^{\prime}
\end{aligned}
$$


If all of the following inequalities hold then the event is inside the collimator-plustolerance region and will be rejected, i.e. $f_{h}(t)=1$.

$$
\begin{aligned}
\left|\tilde{y}_{h}^{f}-y_{o}^{h}\right| & <\left(h_{e}^{h}+t\right) \\
\left|\tilde{x}_{h}^{f}-x_{o}^{h}\right| & <\left(v_{e}^{h}+t\right) \\
\left|\tilde{x}_{h}^{f}-x_{o}^{h}\right| & <\left(\frac{-v_{e}^{h}}{h_{e}^{h}}\left|\tilde{y}_{h}^{f}-y_{o}^{h}\right|+3.0 \frac{v_{e}^{h}}{2.0}+t\right) \\
\left|\tilde{y}_{h}^{r}-y_{o}^{h}\right| & <\left(h_{e x}^{h}+t\right) \\
\left|\tilde{x}_{h}^{r}-x_{o}^{h}\right| & <\left(v_{e x}^{h}+t\right) \\
\left|\tilde{x}_{h}^{r}-x_{o}^{h}\right| & <\left(\frac{-v_{e x}^{h}}{h_{e x}^{h}}\left|\tilde{y}_{h}^{f}-y_{o}^{h}\right|+3.0 \frac{v_{e x}^{h}}{2.0}+t\right)
\end{aligned}
$$

So to summarize $f_{h}(t)$ is piecewise and defined by the above condition for being "inside" the collimator-plus-tolerance.

$$
f_{h}(t)= \begin{cases}1, & \text { inside } \\ 0, & \text { outside }\end{cases}
$$

\subsection{Fiducial and PID Rejection Analysis}

The relevant variables for all data rejections should be given a somewhat closer look. It is useful to know the specifics of all such data processing as it will ultimately determine the integrity of experimental data obtained.

\subsubsection{Time of Flight}

A good method for identifying particles which are of different masses is a time of flight method. One can simply calculate the time of flight for a given particle species and then normalize to obtain the relative flight times of other particle species. In the proton detection experiment this can be done by correcting the time of flight to make the proton appear at a specific time independent of momentum. One can then take a simple interval of the timing spectrum and be reasonably sure that all of the events in that interval are protons. This procedure can be applied to get any final state particle. One can also plot the other possible final state particles 
relative to the normalized particle's time. This will allow one to see how efficient the rejection is likely to be if there is background from other non-signal events.

It is obvious that the velocity of a particle can be simply obtained from its momentum and energy in units of, $c$, the speed of light ${ }^{4}$.

$$
\beta=\frac{|\mathbf{p}|}{\sqrt{|\mathbf{p}|^{2}+m^{2}}}
$$

The inverse of this velocity is related to the time by multiplication of the path length in appropriate units. A correction to account for the momentum-dependent difference in path lengths in a particle spectrometer should be applied if highly accurate results are required. Ignoring the path length correction at first one can simply obtain the inverse velocities (i.e. $\frac{1}{\beta}$ ) as functions of the particle momentum for different particle types. For a few particle types in the vicinity of the mass of the nucleon these functions are the following:

$$
\frac{1}{\beta_{i}}=\frac{\sqrt{|\mathbf{p}|^{2}+m_{i}^{2}}}{|\mathbf{p}|}
$$

The index $i$ can run over all possible particle types. Figure 7.1 lists the inverse velocities of several different particle types. The pion, being the lightest particle, is clearly going nearly the speed of light for all momenta of interest. One can then obtain a time by multiplying the inverse velocities by the appropriate distance. Assuming that the appropriate distance is not momentum dependent for a moment, one can obtain this distance by using the measured time spectrum where there are two clear particle peaks at a given momentum. Particularly one has that the time of flight $t_{i}$ for a given particle is simply related to the path length $s$.

$$
t_{i}(|\mathbf{p}|)=\frac{s}{\beta_{i}(|\mathbf{p}|)}
$$

Although it may be difficult to obtain $s$ analytically because of the bending of the spectrometer paths, it is not too difficult to calculate the absolute time difference

\footnotetext{
${ }^{4}$ The unit system here is natural units where $\hbar=c=1$ and the remaining unit is chosen to be energy in GeV.
} 


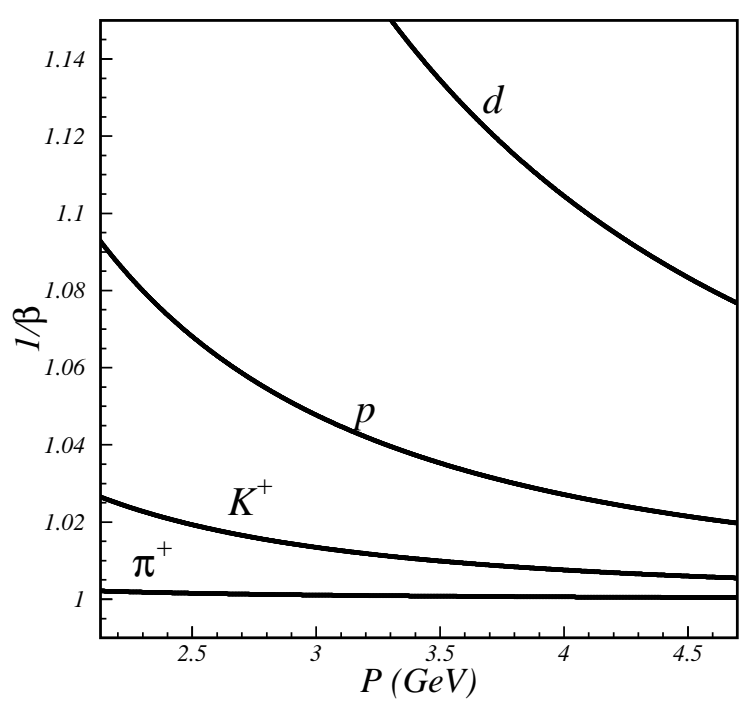

\section{Figure 7.1: The inverse velocities of the deuteron, proton, kaon and pion respectively as functions of momentum $|\mathbf{p}|$.}

between two particle types at a given momentum.

$$
\left|t_{i}(|\mathbf{p}|)-t_{j}(|\mathbf{p}|)\right|=s\left|\frac{1}{\beta_{i}(|\mathbf{p}|)}-\frac{1}{\beta_{j}(|\mathbf{p}|)}\right|
$$

Figure 7.2 shows the coincidence time spectrum for proton and $\pi^{+}$events at particle momentum $3.10 \mathrm{GeV}$. Neglecting the possible path-length differences for these events one can get the value of the parameter $s$ in the appropriate units to give the calculated times the same units as the coincidence spectrum ${ }^{5}$. The only important feature of these times is that the differences in times between two different particles gives the appropriate value. This gives the freedom to define one particular particle as having a time which is independent of momentum and at an arbitrary value, as long as the other times are properly adjusted. This procedure is advantageous because after normalizing to a particular particle, removing other events from the spectrum becomes an easy task which can be done by taking an interval of the spectrum in coincidence time. Mathematically the important feature of the calculated

\footnotetext{
${ }^{5}$ The coincidence spectrum is quoted in a time variable which has more negative times representing longer elapsed times. This has no bearing on the absolute time difference as calculated in Eqn. 7.10 but in order to compare to the coincidence time spectrum a negative sign and overall normalization factor in the $t_{i}=\frac{1}{\beta_{i}}$ will be introduced.
} 


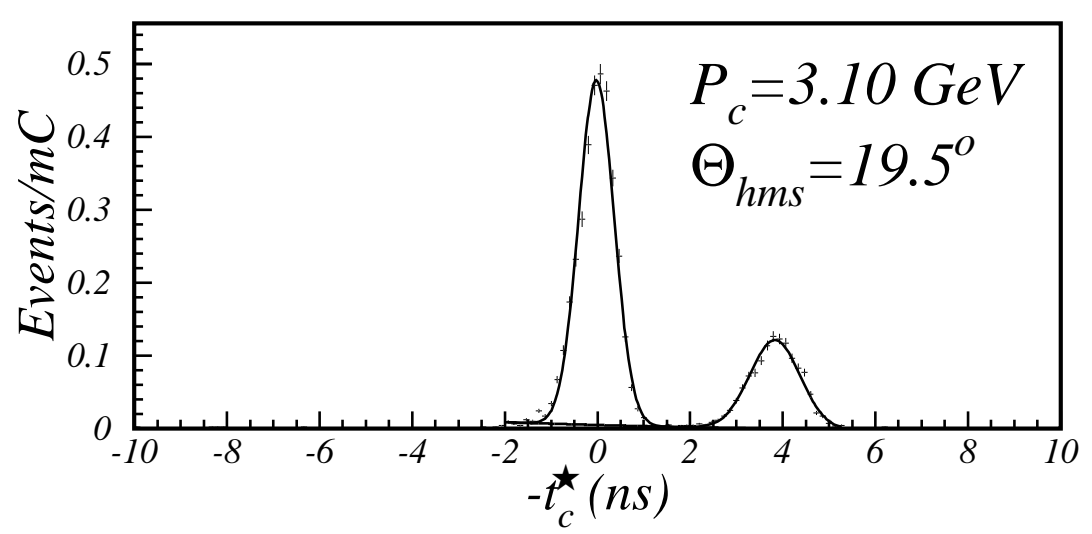

\section{Figure 7.2: Coincidence time spectrum for proton and $\pi^{+}$at $3.10 \mathrm{GeV}$ spectrometer central momentum.}

times (normalizing to the proton time) is:

$$
\left|t_{i}-t_{p}\right|=s\left|\frac{1}{\beta_{i}}-\frac{1}{\beta_{p}}\right|
$$

It is then clear that if one defines $t_{i}^{\star} \equiv\left(t_{p}-t_{i}\right)$ then the following relation holds:

$$
\left|t_{i}^{\star}-t_{p}^{\star}\right|=s\left|\frac{1}{\beta_{i}}-\frac{1}{\beta_{p}}\right|
$$

Which is the same as the defining Eqn. 7.11. With this new time variable we have $t_{p}^{\star} \equiv 0$. Therefore all the relative times are the same but the proton time is defined to be zero for all momenta. This fact can obviously be used to normalize to any particle. The new times can be written easily in terms of the momenta.

$$
t_{i}^{\star}=\frac{s}{|\mathbf{p}|}\left(\sqrt{|\mathbf{p}|^{2}+m_{p}^{2}}-\sqrt{|\mathbf{p}|^{2}+m_{i}^{2}}\right)
$$

The same is obviously true if one normalizes to another particle, for example, the $\pi^{+}$. The new time variable which satisfies Eqn. 7.11 can then be expressed similarly as before.

$$
\tilde{t}_{i}=\frac{s}{|\mathbf{p}|}\left(\sqrt{|\mathbf{p}|^{2}+m_{\pi}^{2}}-\sqrt{|\mathbf{p}|^{2}+m_{i}^{2}}\right)
$$

One can then easily plot coincidence data normalizing to any particle one wishes, in particular the proton or $\pi^{+}$. Making the momentum dependence of the desired 
particle straight will then correspond to a sharper peak for the desired events in a one dimensional coincidence time histogram. The construction of such a histogram allows one to reject all but the desired particle with very high accuracy. Defining the coincidence experimental time as $t_{c}^{\star}$ when normalizing to the proton and $\tilde{t}_{c}$ when normalizing to the $\pi^{+}$one can use the data and theoretical curves above to examine the traces of each particle type. If one is to define the experimental

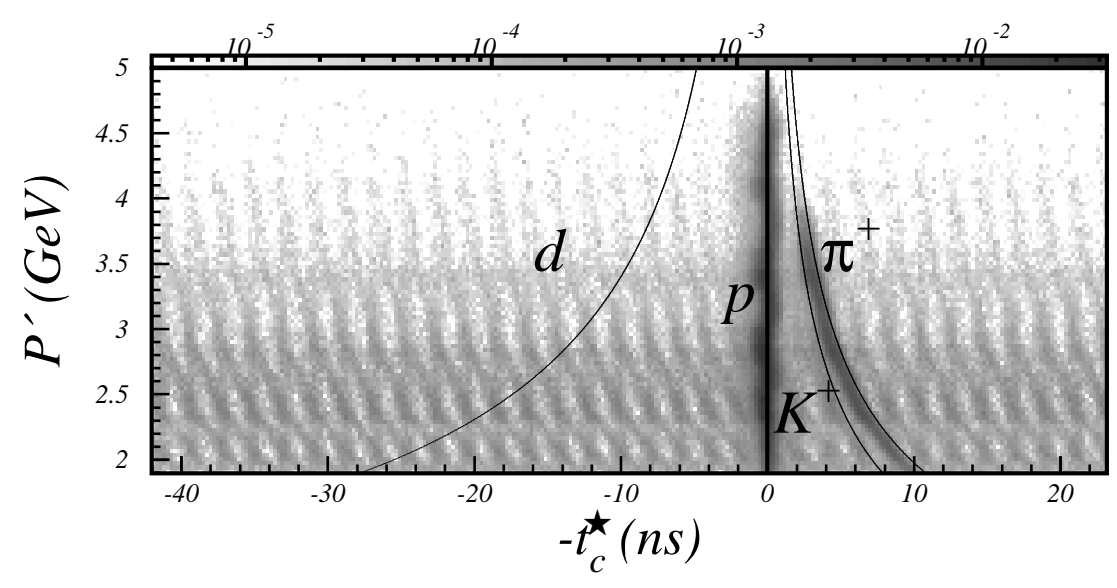

Figure 7.3: $t_{c}^{\star}$ vs. hadron momentum for coincidence data normalized to make the proton vertical.

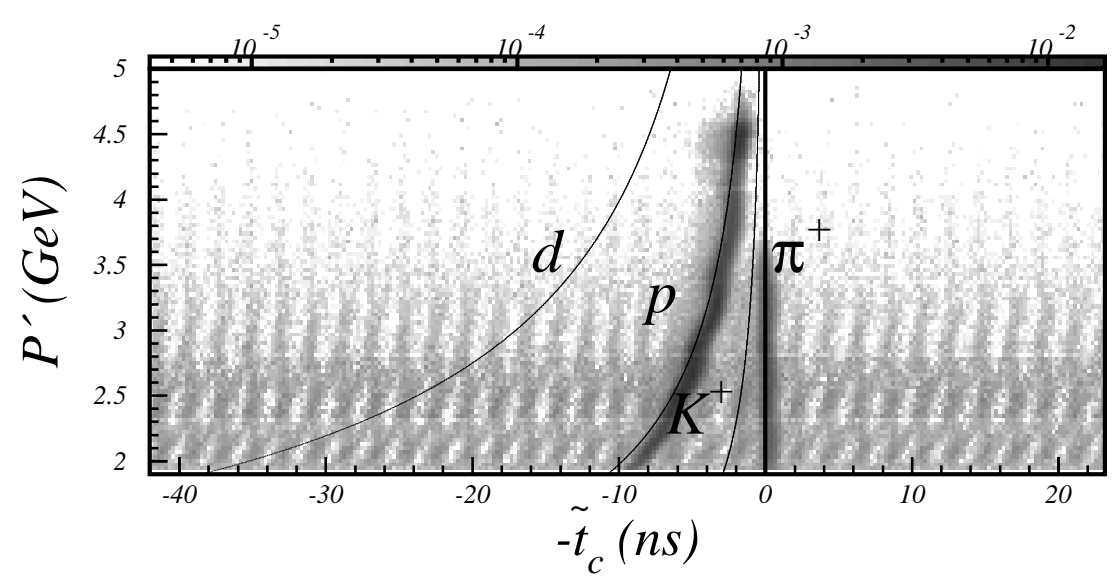

Figure 7.4: $\tilde{t}_{c}$ vs. hadron momentum for coincidence data normalized to make the pion vertical.

variable coincidence time as $t_{c}^{\star}$ when normalized to the proton, then obtaining the 
experimental coincidence time normalized to the $\pi^{+}$is trivial to construct.

$$
\tilde{t}_{c}=t_{c}^{\star}-t_{\pi}^{\star}
$$

By using this procedure the coincidence time variable distribution is made to peak about the time of the particle of interest. The Fig. 7.5 justifies the use of the data

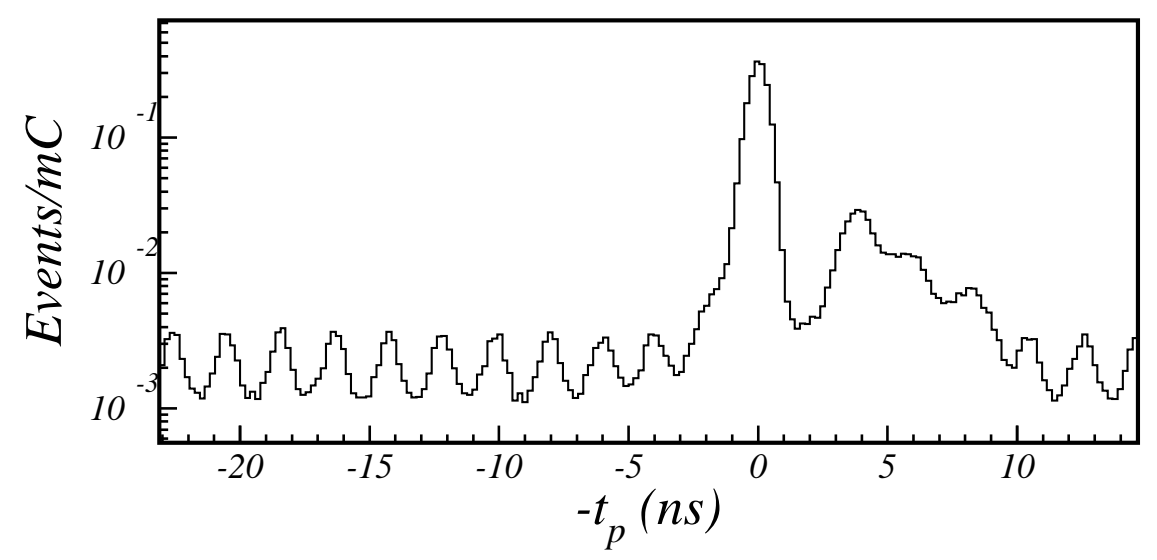

Figure 7.5: $t_{c}^{\star}$ for coincidence data normalized to make the proton peak sharp. The structures visible on the low and high edges of the plot are due to the coincidence events from different $R F$ beam bunches.

restriction mentioned in Eqn. 7.3. Most of the proton peak is captured by this restriction and what is not captured should be included in the systematic analysis of the coincidence time cut postponed until Chapter 10. It should be noted that the above normalization is useful only when the constant $s$ is assumed to have no functional dependence on the momentum p. It is known, however, that $s$ must depend on the momentum as $s(\mathbf{p})$ since the bending spectrometer gives particles with different momenta slightly different paths. It is clear from the previous analysis that this functional dependence is weak at best so that $s(\mathbf{p}) \simeq s_{0}+\epsilon f(\mathbf{p})$, where $\epsilon \ll 1$. The $s$ that was used previously can then be identified with $s_{0}$. If this path length correction were a less negligible effect it may be useful to empirically fit the coincidence time peaks at each momentum to try to extract the function $f(\mathbf{p})$ and thereby obtain a path length correction to the different momentum paths in the spectrometer. This sort of analysis is overkill for the above purpose since the 


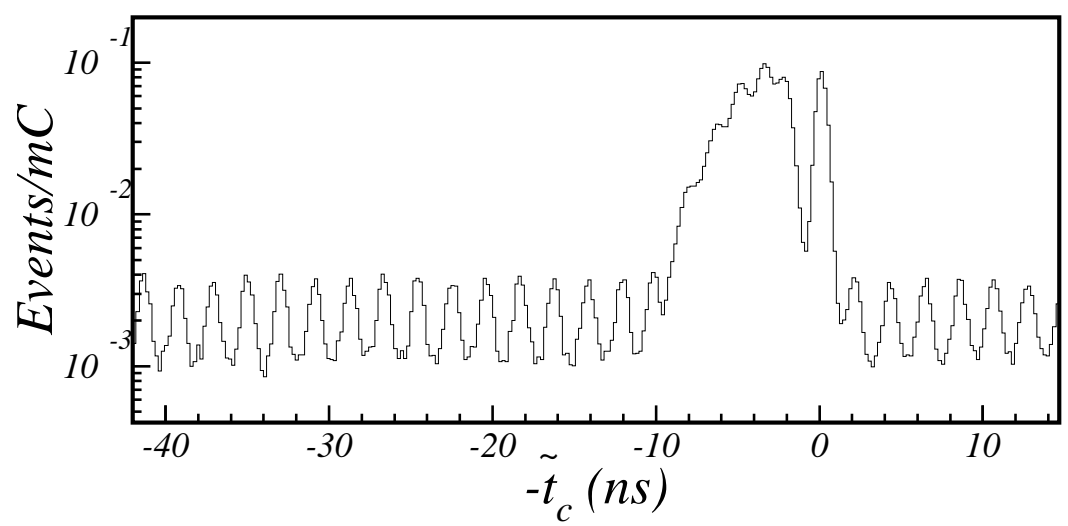

\section{Figure 7.6: $\tilde{t}_{c}$ for coincidence data normalized to make the pion peak sharp.}

separation obtained between proton and $\pi^{+}$events is good in both cases.

\subsubsection{Missing Mass}

Since the interest is neutral pion events and the spectrometer system only measures an outgoing electron and proton, it is clear that energy and momentum conservation must be exploited to identify any other particles present in the final state. The best way to do this in a covariant way is to use the missing mass already introduced as $M_{x}^{2}$. If one defines a missing 4 -momentum as $p_{m}$ then this quantity is the square of that 4 -momentum vector, $p_{m}^{2}=M_{x}^{2}$. If there is only a single missing particle this quantity will be very close to the mass squared of that missing particle and exhibit a sharp (depending on resolution as we shall see) spike. This is how the present experimental analysis will identify pions as opposed to heavier missing mesons ( $\eta$ for example). The simplest way to do the missing mass exclusion is to examine the experimental resolution and then define limits which select most pion events and reject everything else. This is, of course, the most ideal case. Experimental resolutions can vary with the kinematic parameters so that this missing mass peak actually becomes wider in some regions of the measured kinematics. This effect is indeed present in the current analysis. Figure 7.7 shows how one can expect the missing mass resolution to vary as a function of $\theta_{p}^{*}$. This plot was obtained from Monte Carlo simulation of the signal events using a flat 

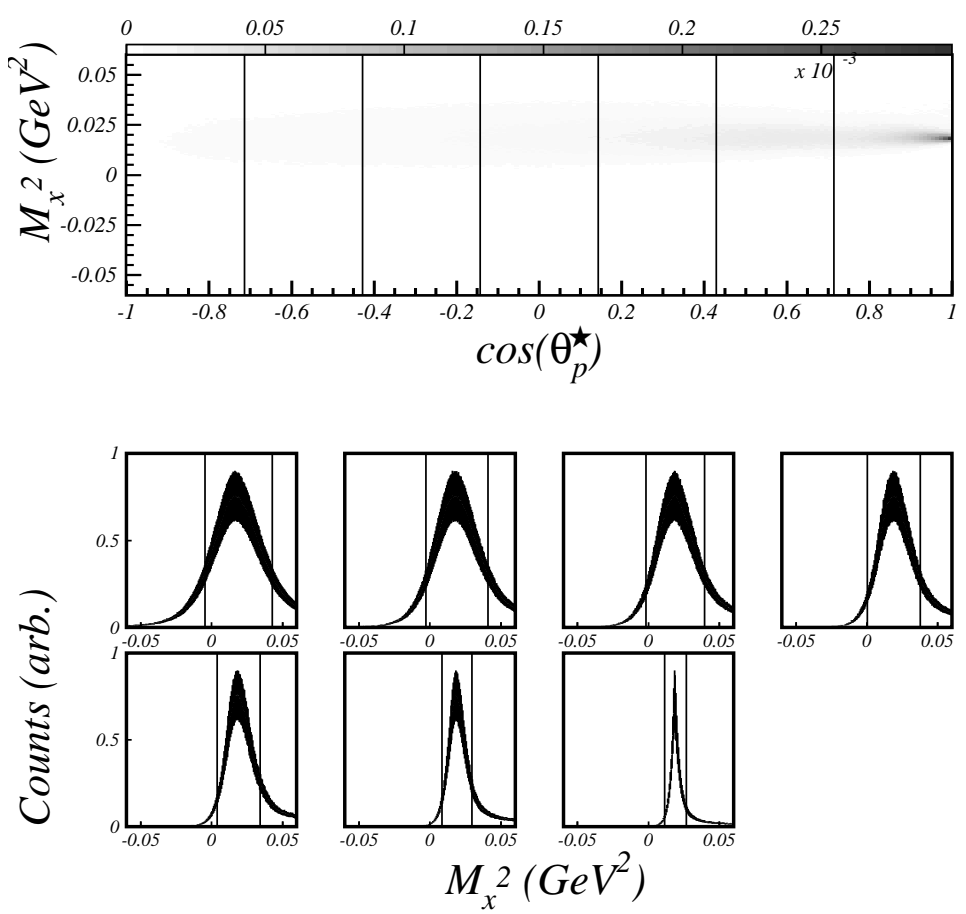

Figure 7.7: Monte Carlo simulation of $M_{x}^{2}$ and $\cos \theta_{p}^{*}$. The vertical lines in the two-dimensional plot signify the boundaries for each of the projections plotted in the lower portion of the figure. The projections progress from left to right and then top to bottom with increasing $\cos \theta_{p}^{*}$. The vertical lines on the projection plots mark the missing mass restrictions used in the analysis.

center of mass virtual photon cross section. The simulation is useful for studying resolutions in different kinematic variables. The two dimensional plot is viewed in the figure along with the slices in the variable $\cos \theta_{p}^{*}$ which clearly show that as the protons become more forward going with respect to the momentum transfer vector, the missing mass distributions become narrower. This is obviously a resolution effect because one can see that the peaks displayed in Fig. 7.7 are centered about $m_{\pi}^{2}=0.019 \mathrm{GeV}^{2}$ and would have infinitesimal width for perfect resolution detectors since by definition the signal Monte Carlo simulation produces neutral pions with $100 \%$ efficiency with respect to any other kind of event. These facts would not be such a problem in an analysis if the Monte Carlo matches the data in resolution at all kinematic points. In that case one would simply make the missing mass restriction a function of the kinematic variables involved. In the present work the 
only important kinematic variable for the missing mass resolution was found to be $\cos \theta_{p}^{*}$ or equivalently $\cos \theta^{*}$. Figure 7.8 shows the data in a similar light as the previous plot. The elastic radiative events have been subtracted and thus the
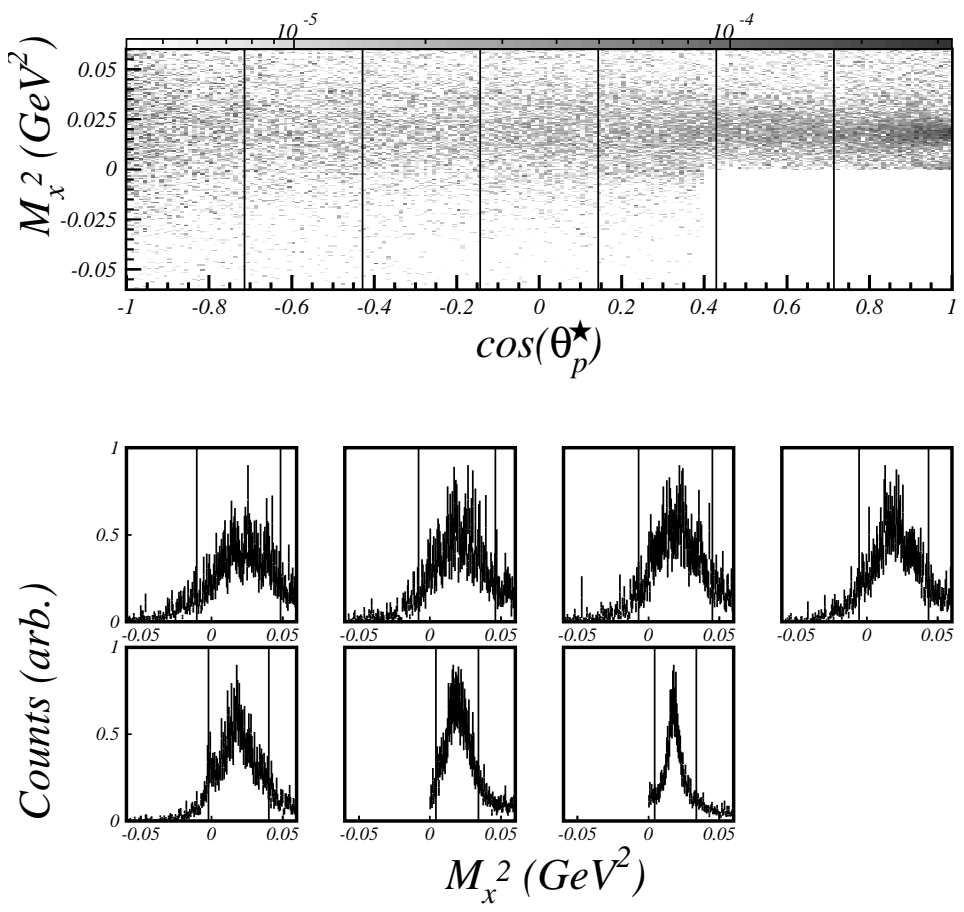

Figure 7.8: Data distribution in $M_{x}^{2}$ and $\cos \theta_{p}^{*}$. The vertical lines in the two-dimensional plot signify the boundaries for each of the projections plotted in the lower portion of the figure. The projections progress from left to right and then top to bottom with increasing $\cos \theta_{p}^{*}$. The vertical lines on the projection plots mark the missing mass restrictions used in the analysis.

appearance of some empty regions in the data distribution. It is clearly seen that this dependence of missing mass resolution on the proton angle (or pion angle) is ever present. Realizing this, from an empirical perspective one can simply check that the resolutions are the same within errors for the Monte Carlo and the data and then apply an angle dependent particle identification exclusion. To check this, the width of the window about $M_{x}^{2}=0.019 \mathrm{GeV}^{2}$ which encloses $80.0 \%$ of the events is examined. This width is actually pictured in each of the Figs. 7.7 and 7.8 but is also displayed in numerical form in Tbl. 7.3. In Tbl. $7.3 c_{m c}$ represents the center of the Monte Carlo distribution and $w_{m c}$ represents its width, a similar convention holds 


\begin{tabular}{|c|c|c|c|c|}
\hline $\cos \theta_{p}^{*}$ range & $c_{m c}\left(\mathrm{GeV}^{2}\right)$ & $w_{m c}\left(\mathrm{GeV}^{2}\right)$ & $c_{d}\left(\mathrm{GeV}^{2}\right)$ & $w_{d}\left(\mathrm{GeV}^{2}\right)$ \\
\hline$-1.0 \leq \cos \theta_{p}^{*}<-0.714$ & 0.019 & 0.0473 & 0.019 & 0.0591 \\
\hline$-0.714 \leq \cos \theta_{p}^{*}<-0.429$ & 0.019 & 0.0439 & 0.019 & 0.054 \\
\hline$-0.429 \leq \cos \theta_{p}^{*}<-0.143$ & 0.019 & 0.0412 & 0.019 & 0.0519 \\
\hline$-0.143 \leq \cos \theta_{p}^{*}<0.143$ & 0.019 & 0.0372 & 0.019 & 0.0492 \\
\hline $0.143 \leq \cos \theta_{p}^{*}<0.429$ & 0.019 & 0.0302 & 0.019 & 0.0427 \\
\hline $0.429 \leq \cos \theta_{p}^{*}<0.714$ & 0.019 & 0.0212 & 0.019 & 0.0300 \\
\hline $0.714 \leq \cos \theta_{p}^{*}<1.0$ & 0.019 & 0.0153 & 0.019 & 0.0292 \\
\hline
\end{tabular}

Table 7.3: Missing mass resolutions.

for data variables and the subscript "d." It is clear that the Monte Carlo simulation systematically underestimates the missing mass resolution. It is good to also plot the behavior of the widths as the cosine of the angle increases to see if there is simply a systematic shift or a true dependence difference. Figure 7.9 shows that the simulation is systematically narrower than the data at all of the points. The last two

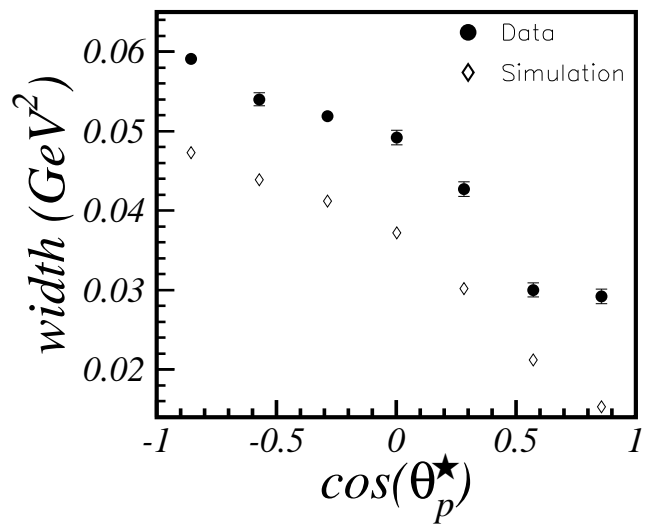

Figure 7.9: Trends of the $80 \%$ widths.

points of the data widths are affected by the elastic radiative subtraction rejection. This is plainly visible in the data distributions of Fig. 7.8. The error associated with this effect will be evaluated as part of the systematic error for the elastic radiative rejection and should not concern us here. The systematic width shift still remains. There are two ways to deal with the situation, one involves modifying the simulation and the second is quantifying the error and including it as part of the systematic error on the extracted cross section. Since the modification of the simulation may 
take a considerable amount of analysis, the error will be quantified here and if the final results can be considerably improved by a modification of the simulation, then the necessary steps will be taken. The final rejection criterion should be chosen according to the data since the width of this rejection is the one which will determine the size of the statistical errors, which are important on a precise measurement and can be included consistently in any extraction routine which involves fitting. This work uses the rejections listed in Eqn. 7.4.

\subsubsection{Electron Calorimeter}

The SOS spectrometer is used to detect electrons. The information gathered from this spectrometer is used to construct the kinematic variables for the center of mass cross section. It is therefore imperative that the events in the SOS be identified affirmatively as electrons. Fortunately there is a good way to do this given the light mass of electrons with respect to many other (negatively charged) particles which could show up in the SOS spectrometer. The SOS calorimeter will measure the ratio of a particles deposited energy to its momentum and the electron is the only particle (which is negatively charged) for which this ratio should be approximately unity. One can clearly see that there is a pronounced peak at considerably less than

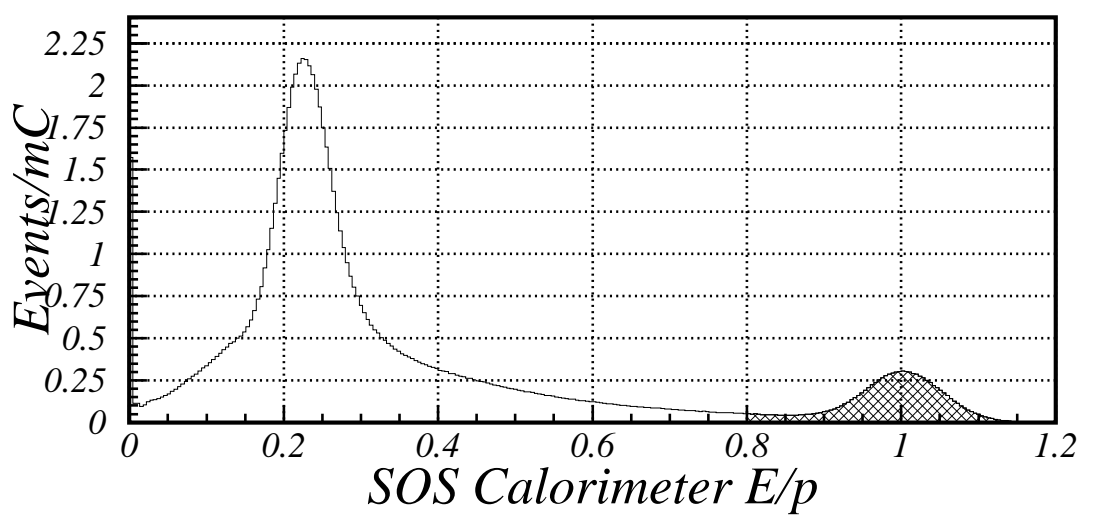

Figure 7.10: Calorimeter electron selection cut. The shaded region corresponds to the accepted events identified as electrons.

unity in Fig. 7.10. This is due mostly to the copious production of $\pi^{-}$particles which can enter into the SOS acceptance fairly easily since the SOS is set to detect 
negatively charged particles. Figure 7.10 shows the data which was retained as the shaded portion of the histogram. This rejection of low $\frac{E}{p}$ events corresponds to the inequality 7.3 in Section7.2, which summarizes the data restrictions.

\subsubsection{Electron Čerenkov}

The SOS Čerenkov detector utilizes Čerenkov radiation to distinguish between particles going faster and slower than light in a gaseous environment. A full service ring-imaging Čerenkov detector will be able to measure a charged particles velocity and thus coupled with a measure of momentum, is a direct constraint on the mass of the particle. The Čerenkov detector can also be used in a "veto mode" which is the use relevant to the present work. No direct measure of the particles velocity is attempted but it is clear that in the present range of momenta, only the lightest particles will radiate appreciably. For the SOS, this means that the requirement of a minimum number of Čerenkov photons will strengthen the electron signal relative to the signal of negatively charged pions. This restriction, coupled to the calorimeter restriction will give a clean electron identification. Figure 7.11 shows the electron

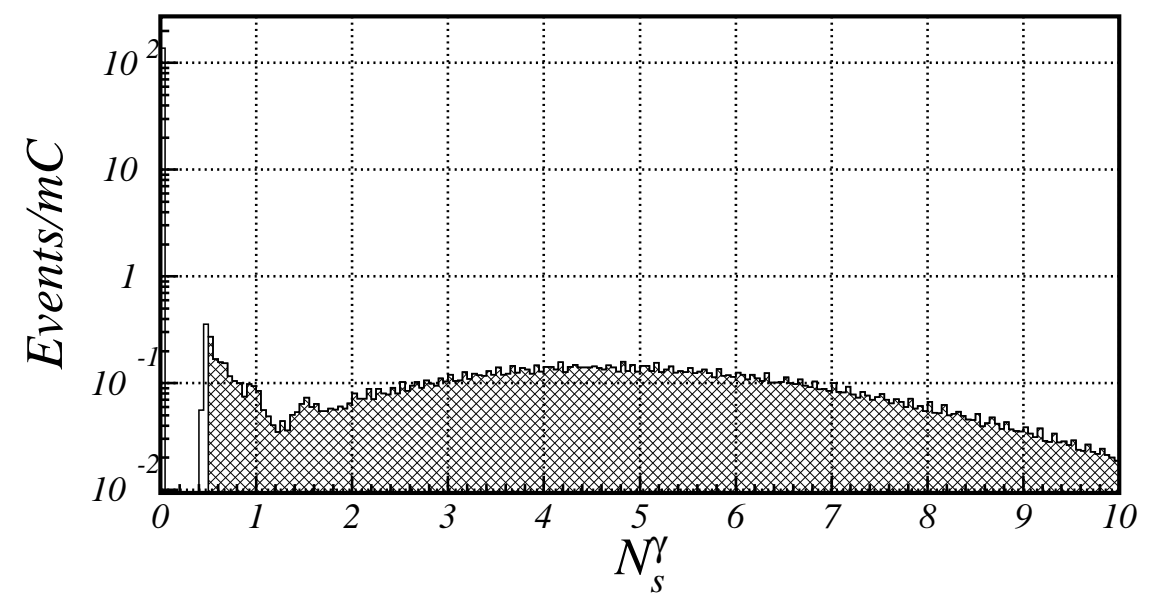

Figure 7.11: Čerenkov electron selection cut. The shaded region corresponds to that which is accepted into the analysis.

selection with the cross-hatched portion of the histogram. The portion of the data that did not fire the Cerenkov is evident in the lowest data bin. An enlargement of this section of the plot is displayed in Fig. 7.12. Most of the events which do 


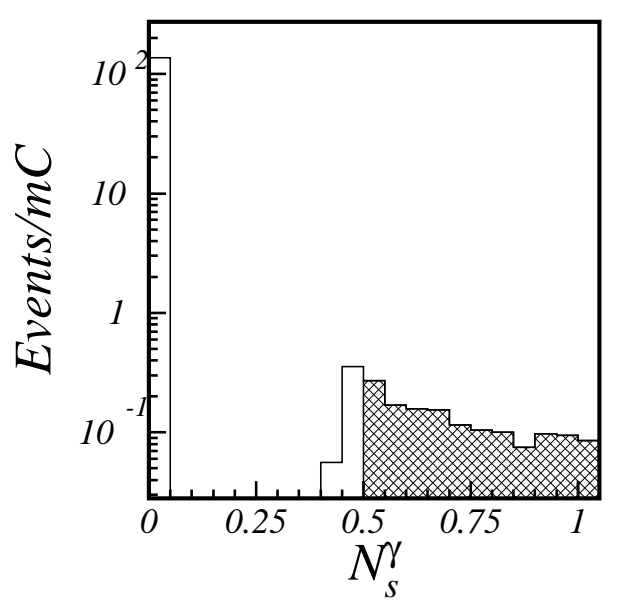

Figure 7.12: Čerenkov electron selection cut pedestal region.

not fire the Cerenkov correspond to $\pi^{-}$events which are evident in Fig. 7.10. The combination of the Čerenkov veto and the calorimeter rejection will identify electrons in the SOS spectrometer to an acceptable percentage. The small percentage of non-electron events should be quoted as a systematic error on these two particle identification restrictions. Figure 7.13 shows the effect of the Cerenkov restriction on the calorimeter spectrum in its accepted region. The curves plotted are a Gaussian and a polynomial background which parameterize the remaining contamination to the electron particle identification. Using the fitted form of Fig. 7.13 one can come

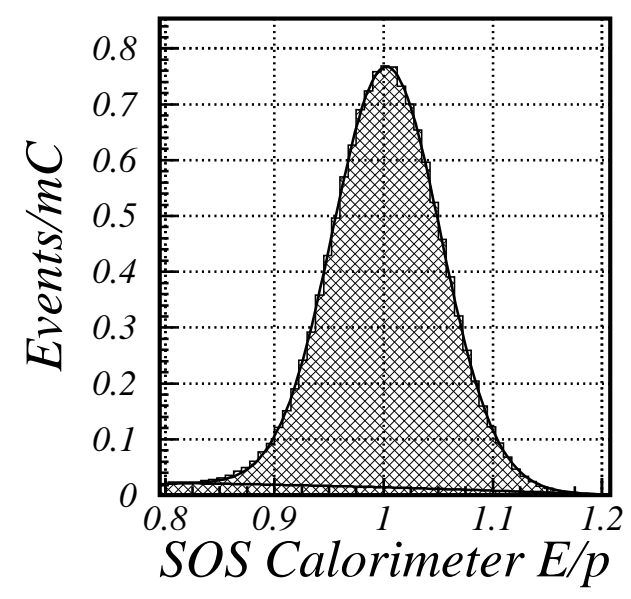

Figure 7.13: Remaining calorimeter contamination.

up with a percent contamination, and use this as a bound for the systematic error 
due to the inefficiency in the electron selection. This value comes out to be $5.3 \%$ according to the above fit. To really estimate how much systematic error comes from this effect one should include other particle identification restrictions. Figure 7.14 shows the calorimeter spectrum after the additional particle identifications for a double arm electron proton measurement. One can then reevaluate the backgrounds

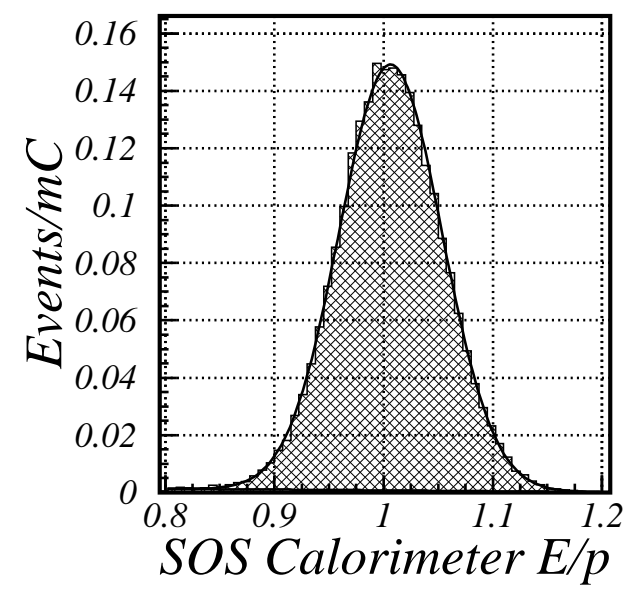

\section{Figure 7.14: Remaining calorimeter contamination with other particle identifications in place.}

to find that the approximate amount of contamination is $1.6 \%$. This value will be included in the calculation of systematic error.

\subsection{Apparatus Backgrounds}

Even with appropriate particle identification restrictions, as have been discussed, there are events present which do not come from the signal process. If left unchecked these events can cause large systematic errors in measured cross sections and therefore in other quantities extracted from the cross sections as well. Often, if knowledge is gathered about non-signal processes one can make effective corrections to extracted data which eliminate the need to place large systematic errors on the final answers or worse, ignoring them. 


\subsubsection{Accidental Counts}

Though the experimental results hinge on measuring the amplitude of a single electron scattering off of a proton, this situation is experimentally unfeasible. In reality accelerated beams of electrons are used which have inherent structure. While this is not much of a problem for inclusive measurements since there is only one particle detected, it can pose a problem for experiments where multiple particles are detected. One must be sure that each of the particles that are detected originated from the same event so as not to lose the kinematic correlation which encodes the scattering amplitudes. Though it is typical of experiments to open a small timing gate upon detection of the first particle, this process is not perfect. Low energy particles from previous events can be produced in such a way as to be detected in the timing gate of the current event. If these "accidentally" detected particles are included with the other particles from the current event, kinematic correlation may be lost. These accidental events must be corrected in order to ensure that the data represents the appropriate process.

The electron beam at Jefferson Lab's continuous electron beam accelerator has a regular periodic structure in time and this structure helps identify contamination from non-vertex electrons or hadrons. The "coincidence time" is a variable which measures when the HMS detector triggers (proton detection) with respect to when the SOS detector triggered. Figure 7.15 shows the distribution of events in this timing variable. The relative scale of Fig. 7.15 is in nanoseconds although the absolute scale is set so that proton events have a coincidence time of zero. Further, the absolute scale is set so that longer times have a more negative value. The prominent proton peak is clearly visible in the timing spectrum, as is the periodic structure of the beam (approximately $2 \mathrm{~ns}$ period) away from zero relative time. Using this periodic structure one can correct for the radio frequency electron bunches which show up under the prominent proton coincidence peak. The specific way in which this correction can be affected is by analyzing the regular structure on the wings of the coincidence time spectrum. The distribution of these events in the other kinematic variables provides us with the number of events that should be removed from each kinematic bin, thereby correcting physically measured quantities such as 


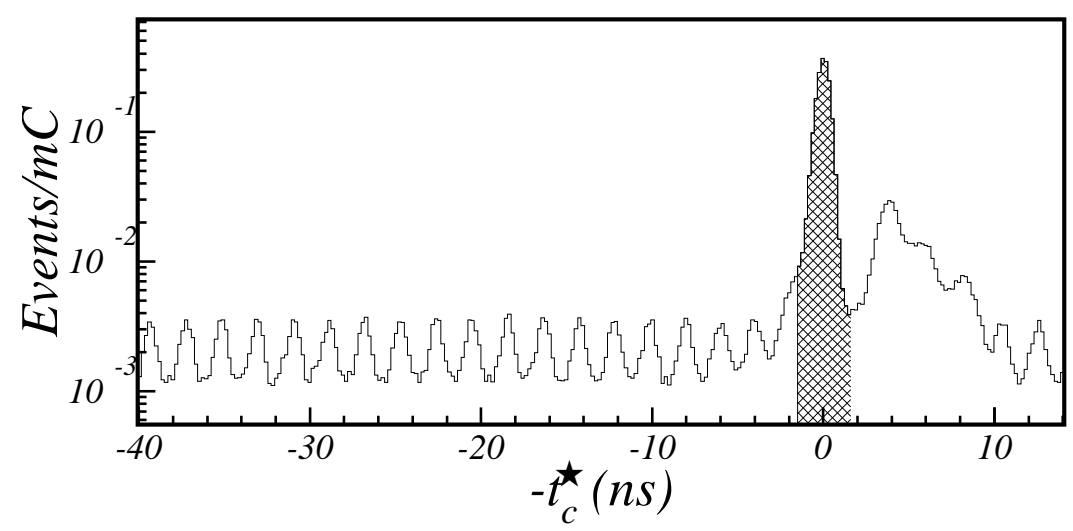

Figure 7.15: Timing spectrum of coincidence events. The shaded insert shows the typical analysis cut made on this spectrum.

cross sections. Figure 7.16 shows the selected timing region in which the distribution has a simple periodic shape (with no coherent contamination like a $\pi^{+}$detected in HMS). In the timing spectrum a secondary shoulder on the positive side of the main

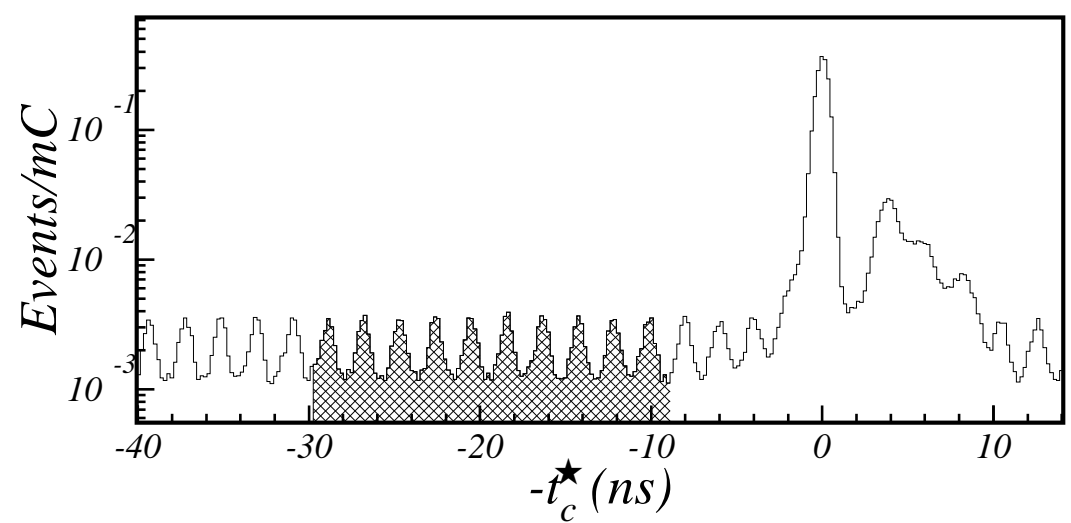

Figure 7.16: Timing spectrum of coincidence events. The shaded insert shows the wing of the timing distribution.

proton timing peak is clearly seen. The obvious interpretation of this peak is that this can represent valid coincidence events which have the correct vertex timing but possibly with a hadron detected in the HMS which is not a proton ${ }^{6}$. It is worth looking at the coincidence time spectrum as a function of HMS detected momentum. Since the relative time dependence on momentum is easily derived one can hope to

\footnotetext{
${ }^{6}$ The polarity of the HMS magnets is set to accept only positively charged particles so $\pi^{+}$and $K^{+}$particles are the most likely candidates
} 
see large distortions in the spectrum with clear momentum dependence for positive hadrons detected in the HMS detector. This dependence can be used (and essentially is) for particle identification but care should be taken that these events do not get confused with ones determination of the periodic beam structure. Moreover, the events used to perform the accidental correction should not include any coherent contribution from vertex hadrons which are simply not protons. Figure 7.3 shows the momentum dependence of the coincidence time spectrum.

The proton has been consistently normalized to appear at the zero point for all momentum. Therefore, the curves in Fig. 7.3 represent the momentum dependence of the denoted particles with the same normalization applied to them as the proton ${ }^{7}$. It is now easy to see the origin of the shoulder in Figs. 7.15 and 7.16. The shoulder is due to a large amount of $\pi^{+}$events $^{8}$. Some other example particles in the mass range from the $\pi^{+}$mass to the deuteron mass have their momentum dependencies plotted in Fig. 7.3. When analyzing the beam structure for the accidental subtraction it is important to use regions of the coincidence time spectrum which are free of other hadronic contaminants. Using the structure from the side where coincidence time is negative and far away from the deuteron curve is sufficient. A more sophisticated extraction of the underlying beam structure might have been warranted if there were higher rates for these other positively charged hadrons. The accidental count subtraction was typically small, $<1.0 \%$, with the worst case being $8.0 \%$ which occured in only one kinematic bin.

\subsubsection{Target End Cap Counts}

Though the experiment is interested in electron scatters off of the liquid hydrogen target, this target material must be contained by material which is obviously not immune to interaction. As a consequence, the data will include interactions that happen within the target walls but these should not be included in the signal process. Since there is a low probability for multiple scatters of a high energy electron beam in a small $\sim 10 \mathrm{~cm}$ length, one can expect most of the target window

\footnotetext{
${ }^{7}$ It is clear that with the normal physical dependence of these curves the only particles which should have no momentum dependence are massless particles. Furthermore, all other massive particles should asymptote from the left.

${ }^{8}$ So large in fact that there is hope that the data may be used for some $\pi^{+}$production physics
} 
events to come from the end caps of the target, just where the beam enters and exits. A simple diagram of the target wall event geometry is given in Fig. 7.17 for a typical spectrometer configuration from the present experiment. If one takes an

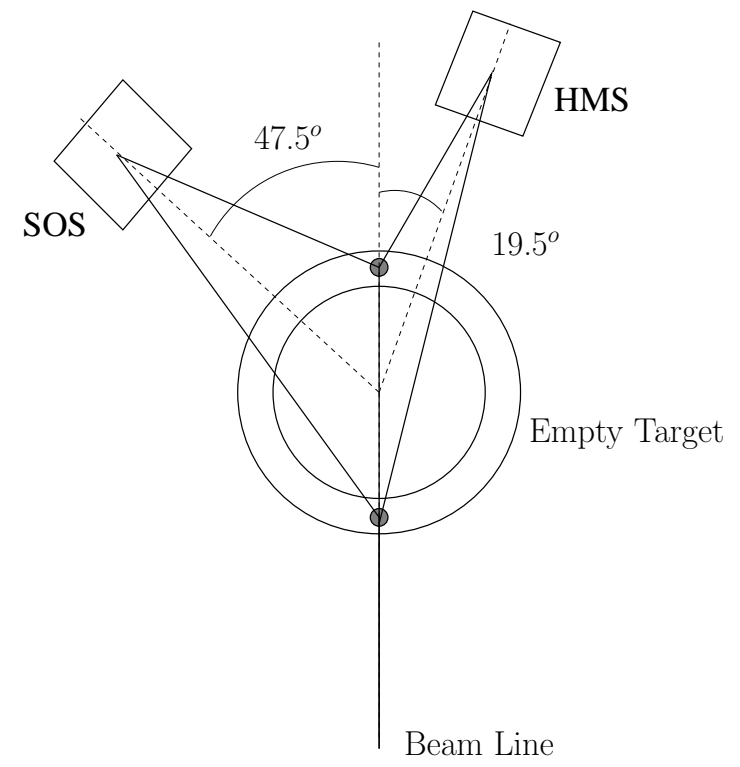

Figure 7.17: Target window event geometry.

experimental run with only a dummy target (target wall but no target material) the end caps should be readily visible. Given the spectrometer coordinate systems defined in Section 5.4 it is easy to see that the planer (or "y") coordinate of the target variables is where this effect should be most easily seen. Figures 7.18 and 7.19 show the count distributions for the target " $y$ " positions for each of the spectrometers. The setting used in producing these plots is $\theta_{S O S}=47.5^{\circ}, \theta_{H M S}=19.5^{\circ}$ and $p_{H M S}=4.5 \mathrm{GeV}$. The angular difference between the spectrometers is evident in the apparent distance between the two end cap peaks. The HMS is set at a smaller angle and therefore perceives the $y_{h}$ separation of the end caps as much smaller. This effect shows that the target window events come from the end caps. One could just remove these events but because of finite resolution the full end cap subtraction can never be affected by such a removal without removing valuable data. Since the contribution of the target walls is found to be very small in coincidence experiments, and in particular this experiment these counts will not be explicitly subtracted but their size will be quoted as a systematic error. Measurements suggest that with 


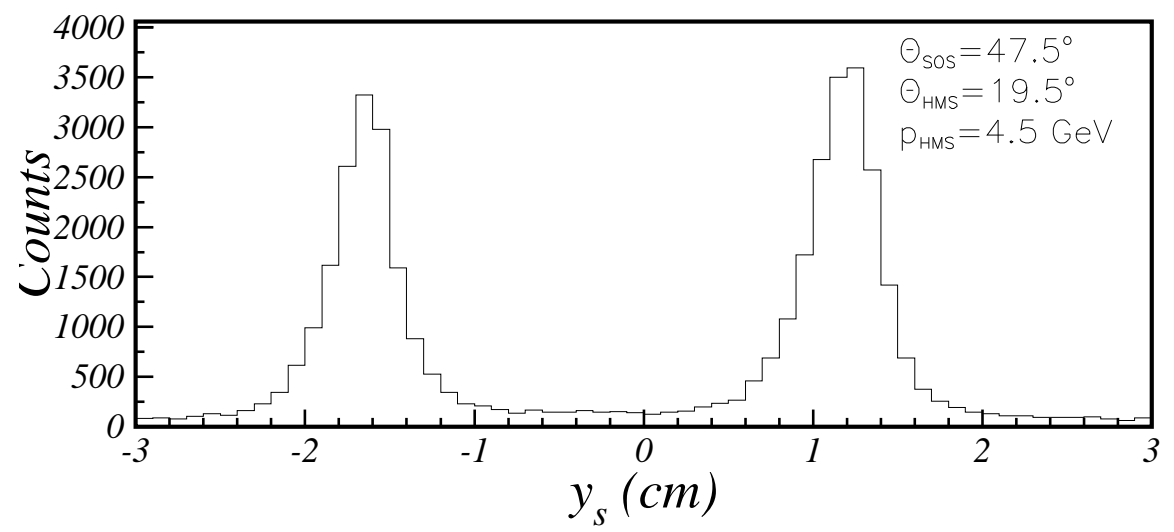

Figure 7.18: Target window counts from the SOS.

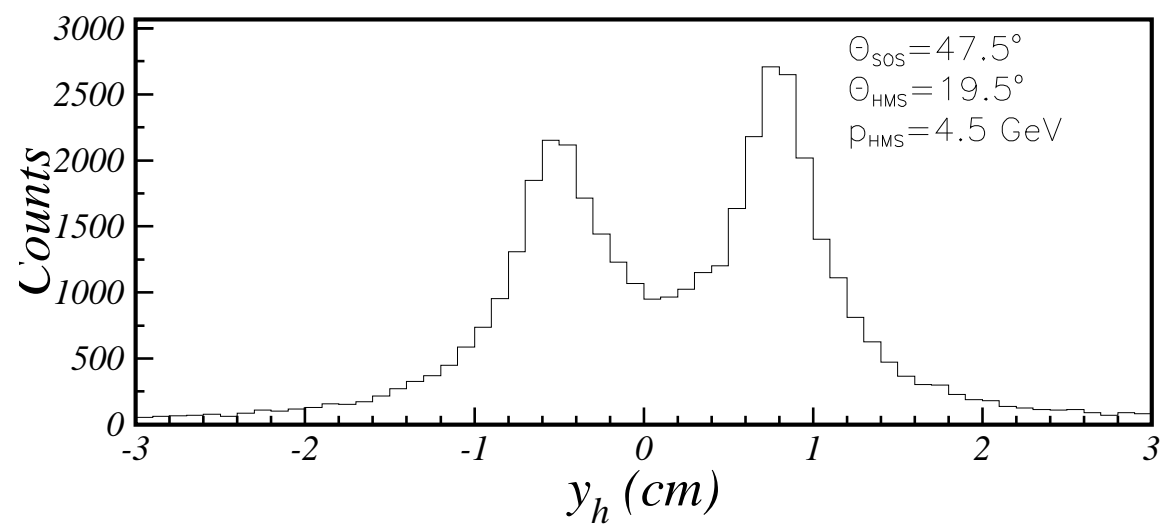

Figure 7.19: Target window counts.

appropriate particle identification restrictions it is typical to have a contribution of $0.5 \%$ in any given kinematic bin $\left(W, \cos \theta^{*}, \phi^{*}\right)$ with the worst case being bounded by $1.0 \%$. Because of the smallness of this contribution for a coincidence measurement, a subtraction will not be done in the current work but a small systematic error will be included.

\subsection{Elastic Radiative Corrections}

There is a final restriction that must be made on the given data which relates to the removal of elastic radiative events which can (because of resolution effects) encroach on the active missing mass region for pions. It was mentioned in Sections 2.3 and 4.2 that there are particular features of this type of process which are 
good differentiators for its rejection. In this section particular use will be made of the fact that this sort of process will come out at values of $\phi^{*}$ very near zero ${ }^{9}$.

The theoretical aspects of calculating reliably the radiative contribution to the elastic cross section are treated elsewhere [36]. The implementation of such equations, however, has a few more considerations to take into account. Owing to varying spectrometer resolutions and acceptances a simulation approach could be the most fruitful. The hope with this approach is that the simulated distribution is representative of the analytical expression within a good approximation. It is wise, however, to begin with an optimized data selection scheme which attempts to remove as much elastic bremsstrahlung as possible. It is therefore useful to first investigate this scheme before using known physics and simulation.

\subsubsection{Elastic Radiative Data Removal}

The effects of elastic radiative events can easily be seen in both the missing mass and $\phi^{*}$ distributions. The difficulty in subtracting these apparent events is the fact that one inevitably must also remove some $\pi^{0}$ events which are important to the extraction of pion production cross section. Luckily, there are some properties of the elastic radiative events such as their tendency to be in plane, which can be utilized.

The distribution of missing mass squared, $p_{m}^{2}$, can be examined along with the $\phi^{*}$ distribution to view the elastic radiative events. The radiative events can be seen spreading out to all missing mass near the zero of $\phi^{*}$ as well as a large concentration near missing mass of zero. These are essentially the events which should be disposed of but one should keep in mind that the full angular coverage cannot be sacrificed for it is this feature that allows the extraction of cross sections over all center of mass angles. In Fig. 7.21 one can see that the full angular coverage is realized somewhat independently of the elastic radiative events, which can be seen faintly concentrated along the line $\phi^{*}=0$. The problem at hand is then to extract these elastic radiative events while disturbing the angular coverage only minimally. Firstly, it will be

\footnotetext{
${ }^{9}$ Note that $\phi^{*}$ is a variable calculated anticipating pion production. It need not have any particular interpretation for other types of processes. Nevertheless $\phi^{*}$ will be small for elastic radiative events. Examining the kinematics figures from Chapter 2 more carefully the reader can justify this fact.
} 


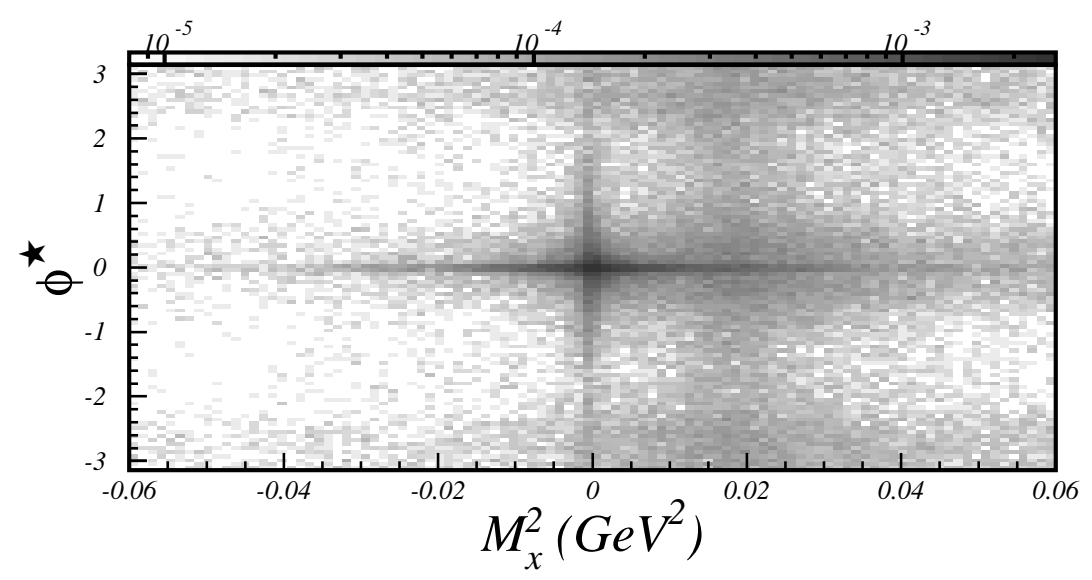

Figure 7.20: $M_{x}^{2}$ vs. $\phi^{*}$ with only standard cuts and $W>1.1 \mathrm{GeV}$ applied.

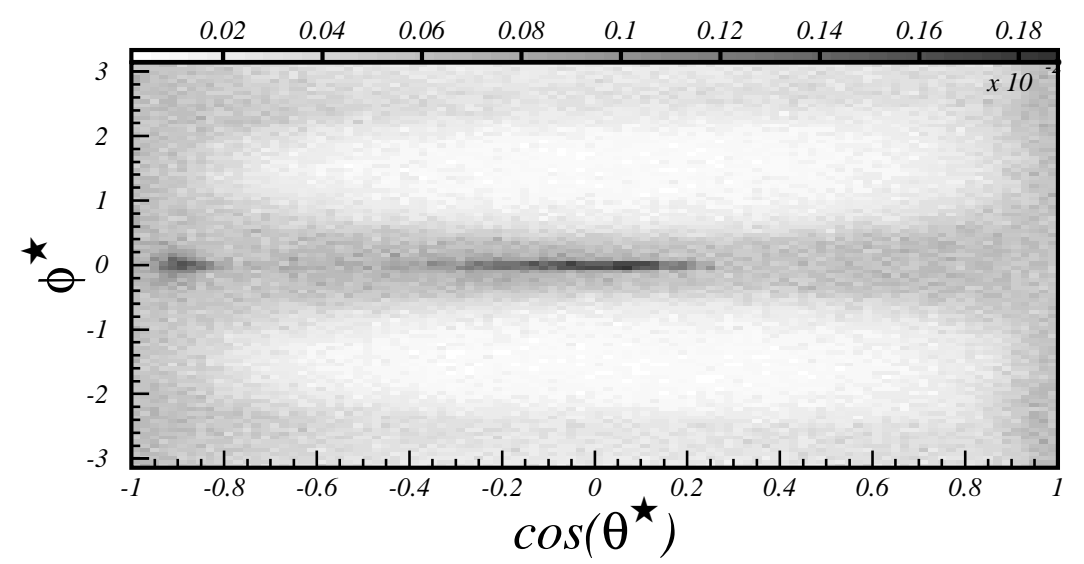

Figure 7.21: $\phi^{*}$ vs. $\cos \theta^{*}$ with only standard cuts and $W>1.1 \mathrm{GeV}$ applied.

useful now and in the future to use a change of variables when displaying the center of mass angular coverage. Since the variables used to describe the center of mass angle are exactly those that appear in a three dimensional coordinate system with a fixed radius, it would seem natural to project the distributions in these angles onto a sphere. The prospect of plotting in three dimensions is, however, undesirable because it will complicate plots. Therefore, the use of polar coordinates on a two dimensional plot seems to strike a balance between naturalness and feasibility. To accomplish this task define the following variables which will reflect the periodicity of $\phi^{*}$, though, will suppress the periodicity of $\theta^{*}$ which is represented as a "radius." It is in some sense natural to retain the periodicity of $\phi^{*}$ since unpolarized pseu- 
doscalar meson cross sections have definite symmetry in this variable whereas in $\theta^{*}$ any symmetry would rely heavily on the dynamics of the situation rather than the kinematics.

$$
\begin{gathered}
X_{\Omega} \equiv\left(\cos \theta^{*}+1\right) \cos \phi^{*} \\
Y_{\Omega} \equiv\left(\cos \theta^{*}+1\right) \sin \phi^{*}
\end{gathered}
$$

When the angular coverage in the center of mass is plotted in terms of the two dimensions $X_{\Omega}$ and $Y_{\Omega}$ a circular pattern is produced with the polar angle representing the $\phi^{*}$ variable and the radius related to $\theta^{*}$. In this way, if the circular distribution is filled with data, full angular coverage has been obtained in the center of mass. Figure 7.22 shows that without any data filtration the data has full angular coverage but has many elastic radiative events which populate the positive $X_{\Omega}$ axis.

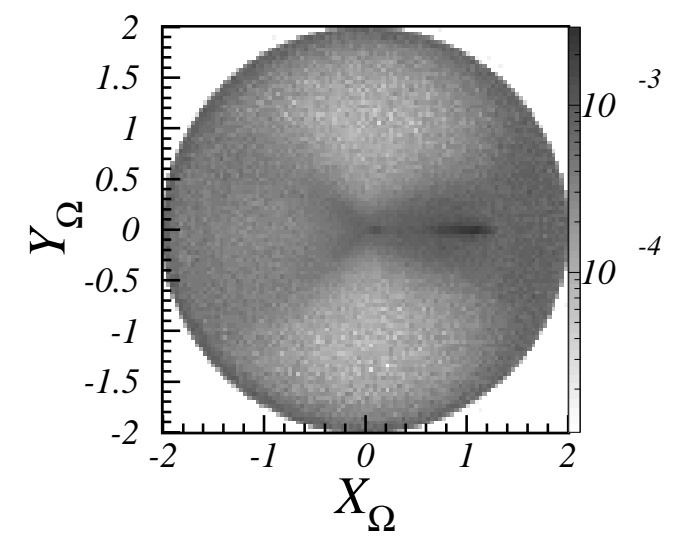

Figure 7.22: $X_{\Omega}$ vs. $Y_{\Omega}$ with only standard cuts and $W>1.1 \mathrm{GeV}$ applied.

Since the majority of the radiation is emitted along the beam and scattered electron directions (see for example peaking approximation in [68]) one can attempt to isolate it according to the angle of the emitted "radiation." Plotting the cosine of the angle of the emitted radiation angle can be fruitful in this way. Unfortunately using the radiation angle in Fig. 7.23 as the basis for exclusion of radiative events seems to be inefficient due to an apparent correlation with the all important variable $\cos \theta^{*}$. When this cut is applied to the data the distribution in Fig. 7.24 is produced. This is clearly not acceptable from the standpoint of angular distributions in the center of mass system. For this reason one can resort to an empirical filter using the $\phi^{*}$ vs. $p_{m}^{2}$ distribution. The band near $\phi^{*}=0$ can be eliminated by a simple 


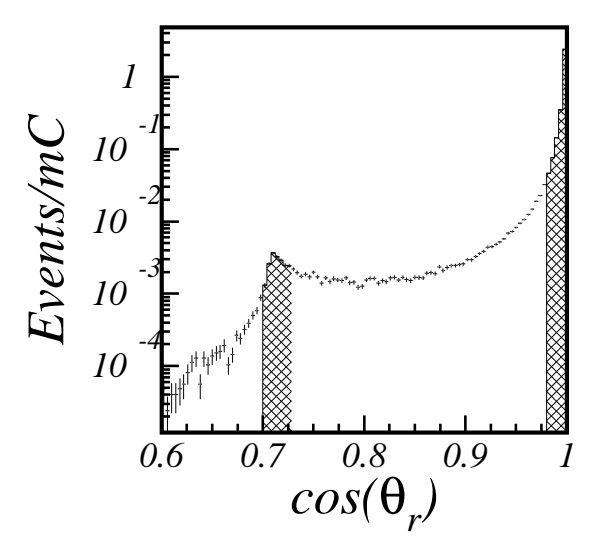

Figure 7.23: Cosine of the angle of emitted radiation and a displayed possible rejection area.

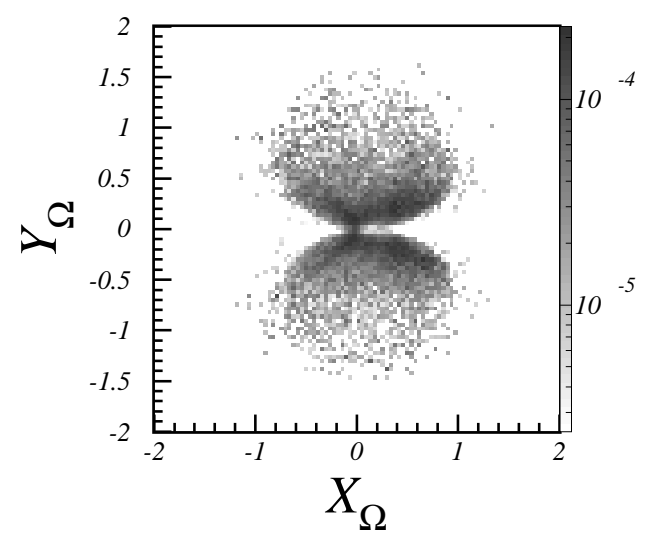

Figure 7.24: $X_{\Omega}$ vs. $Y_{\Omega}$ with restriction on radiation angle and $W>1.1$ GeV applied.

removal of events which are too close to planar. Obviously, meson events can also be produced near $\phi^{*}=0$ but the distribution of radiative events in this region is necessarily much narrower and the hope is that the number of meson events will be accounted for when Monte Carlo comparisons are used to extract the cross section. Actually, a slightly more intricate two dimensional cut can be very effective in eliminating most of the elastic radiative contribution. Examining Fig.7.20 more closely, one can see that the largest part of the elastic radiative contribution is concentrated near $M_{x}^{2}=0$ and $\phi^{*}=0$ with tails extending along the lines with $\phi^{*}=0$ and $M_{x}^{2}=0$. Figure 7.25 is a first attempt to reject some of the elastic radiative background. If one restricts oneself to the larger $M_{x}^{2}$ side of the line 
displayed in the figure, most of the elastic radiative events will be cast aside. The

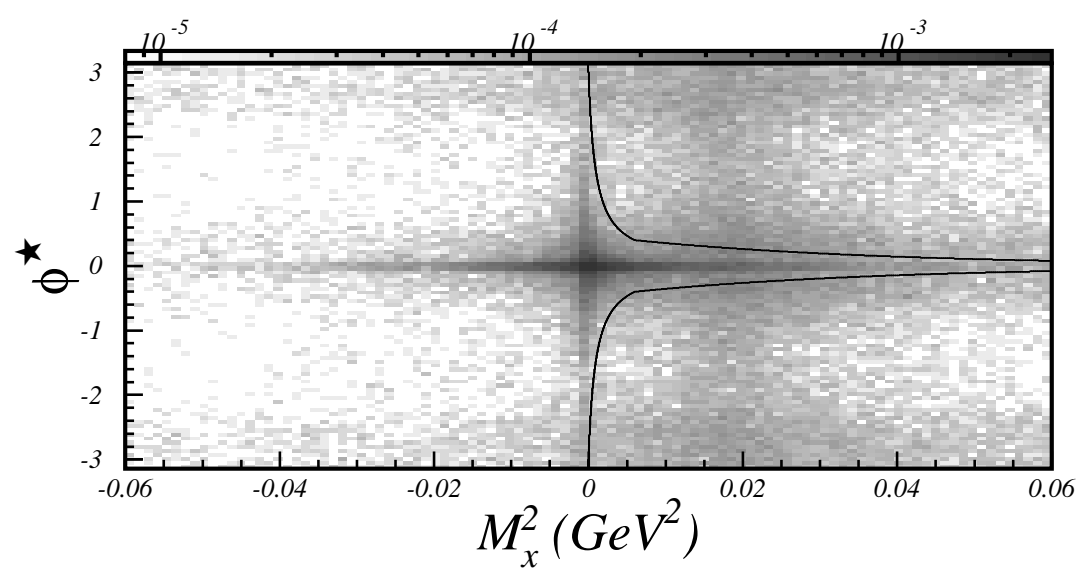

Figure 7.25: $M_{x}^{2}$ vs. $\phi^{*}$ with $W>1.1 \mathrm{GeV}$ and a possible elastic rejection superimposed.

parameterization of this line is accomplished by the use of two exponential functions of $M_{x}^{2}$. First one chooses a point $m_{t}$ at which to terminate the behavior of the first exponential and begin the behavior of the second. The Fig. 7.25 has $m_{t}=0.006$ $\mathrm{GeV}^{2}$. The next step is to define a point $m_{g}$ at which $\phi^{*}$ must have a certain value specified by $\phi_{g}$. The full cut is then constrained by specifying the $\phi^{*}$ value at $M_{x}^{2}=m_{t}, \phi_{t}$, and the decay rate, $\gamma$, of the exponential which takes over for $M_{x}^{2}>m_{t}$.

$$
\left|\phi^{*}\right|= \begin{cases}\left(\frac{\phi_{g}}{e^{-\gamma^{\prime} m_{g}}}\right) e^{-\gamma^{\prime} M_{x}^{2}}, & M_{x}^{2}<m_{t} \\ \left(\frac{\phi_{t}}{e^{-\gamma m_{t}}}\right) e^{-\gamma M_{x}^{2}}, & M_{x}^{2} \geq m_{t}\end{cases}
$$

With the definition:

$$
\gamma^{\prime} \equiv\left(\frac{1}{m_{t}} \ln \frac{\phi_{g}}{\phi_{t}}\right)
$$

The rejection does a reasonably good job, but as was noted in Section7.3.2, the missing mass resolution for meson events gets considerably wider with decreasing $\cos \theta_{p}^{*}$ or increasing $\cos \theta^{*}$. It is then logical to try to retain the same form of the cut but make it vary as a function of $\cos \theta^{*}$. This is logical because the above form seems to be able to reject based on the most salient radiative signatures of the $\phi^{*}$ vs. $M_{x}^{2}$ plots that have been examined so far. Suppose the rejection parameters are allowed to change depending on which of the three $\cos \theta^{*}$ bins from Tbl. 7.4 the 
events fall in. The boundaries in the table were chosen empirically in order to sepa-

\begin{tabular}{|c|c|c|c|c|c|}
\hline $\cos \theta^{*}$ range & $\phi_{t}$ & $\phi_{g}$ & $\gamma$ & $m_{t}\left(\mathrm{GeV}^{2}\right)$ & $m_{g}\left(\mathrm{GeV}^{2}\right)$ \\
\hline$-1.0 \leq \cos \theta^{*}<-0.4$ & 0.4 & 3.0 & 30.0 & 0.006 & 0.0 \\
\hline$-0.4 \leq \cos \theta^{*}<0.25$ & 0.19 & .20 & 20.0 & 0.006 & 0.0 \\
\hline $0.25 \leq \cos \theta^{*} \leq 1.0$ & - & - & - & - & - \\
\hline
\end{tabular}

Table 7.4: Radiative rejection in different $\cos \theta^{*}$ bins.

rate the behaviors that one will see in following plots. There is no apparent radiative elastic contribution in the last $\cos \theta^{*}$ listed in the table and therefore there will be no rejection in this bin. Any radiative elastic contribution to this bin must be either very small or impossible to separate from the important meson production events. The first parameterization listed in the table is the same as was superimposed on Fig. 7.25. Figures 7.26, 7.27 and 7.28 show the overlayed empirical rejections and the separated $M_{x}^{2}$ vs. $\cos \theta^{*}$ plots. This rejection method removes a large por-

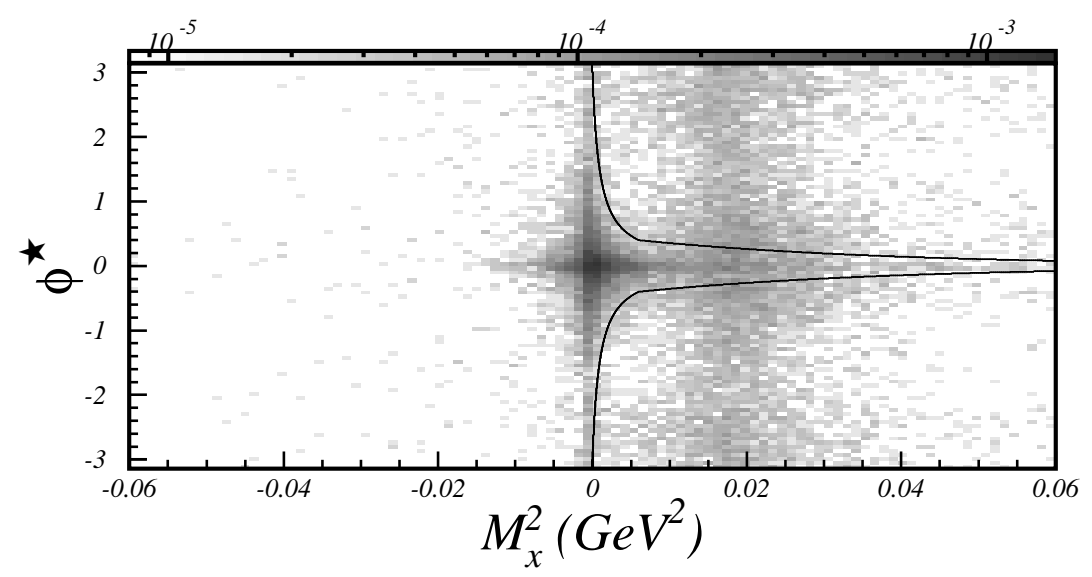

Figure 7.26: Radiative rejection overlay for lowest $\cos \theta^{*}$ bin.

tion of the elastic radiative contamination and removes comparatively little meson production data. Of course, the fact that it removes any pion production data at all signals the need for a correction, this correction will be applied implicitly in the cross section extraction methods of Chapter 8. The basic idea is to extract the physical cross section only by comparison to a phase space simulation of the meson production (to eliminate acceptance). Given this method, if one makes the identical restrictions in the phase space simulation, a reliable extraction should follow. 


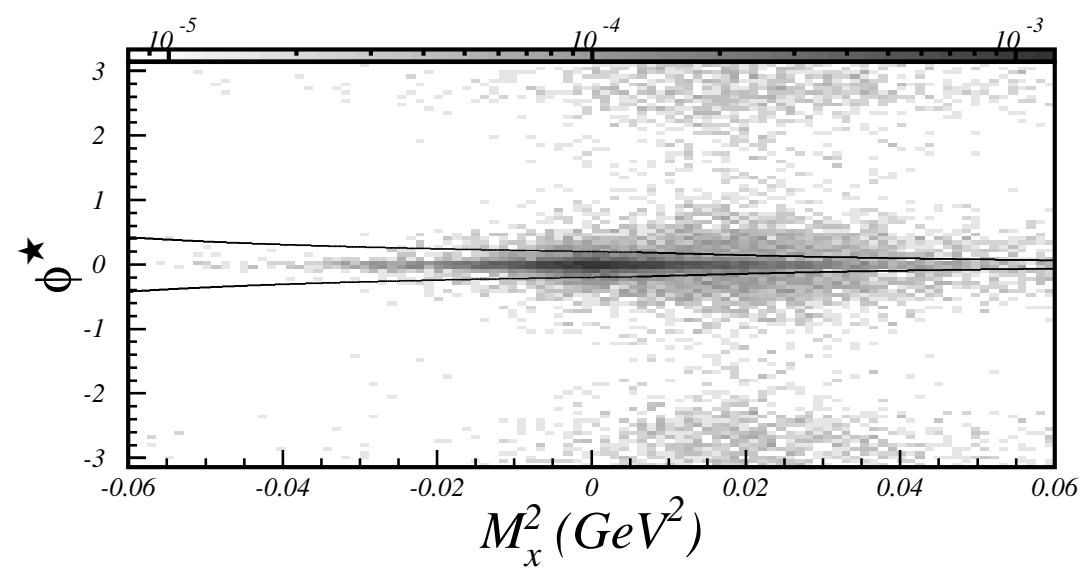

Figure 7.27: Radiative rejection overlay for intermediate $\cos \theta^{*}$ bin.

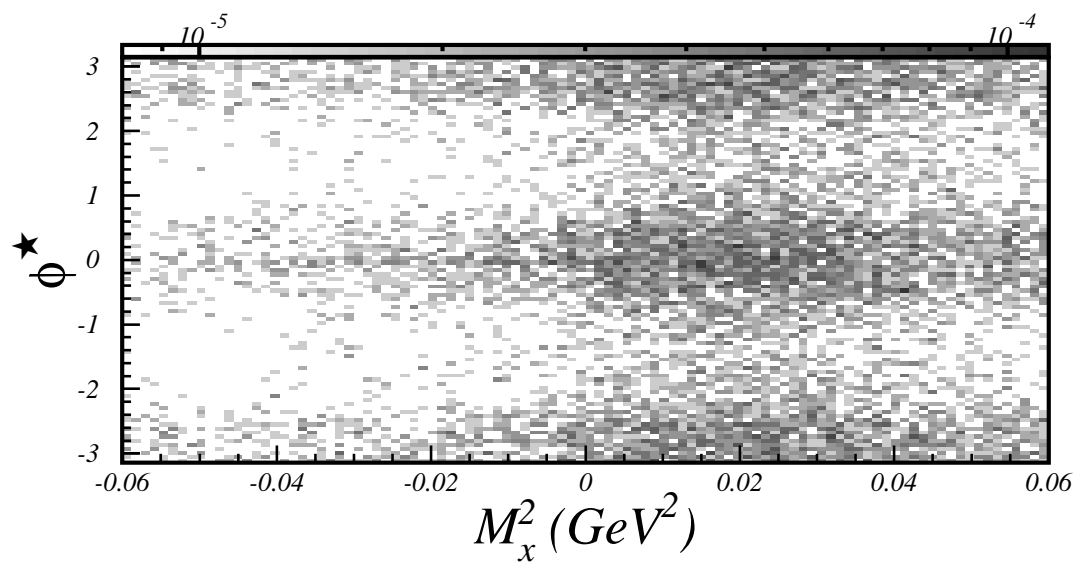

Figure 7.28: Radiative rejection overlay for highest $\cos \theta^{*}$ bin.

Figure 7.29 shows that the angular coverage has been retained for the experiment, after the application of the radiative elastic rejection. This is therefore an acceptable method for the partial elimination of the radiative background. As with all other particle ID or physics data restrictions, it is useful to know how efficient these are and how much systematic error is introduced. For the restriction at hand this is a difficult question, and the best answer can be formed via a sensitivity study of the final answer with respect to small variations in this rejection. This study is postponed until Chapter 10.

In summary, the radiative elastic events have been removed from the current data set via a restriction on the kinematic variables. Since the missing mass spectra 


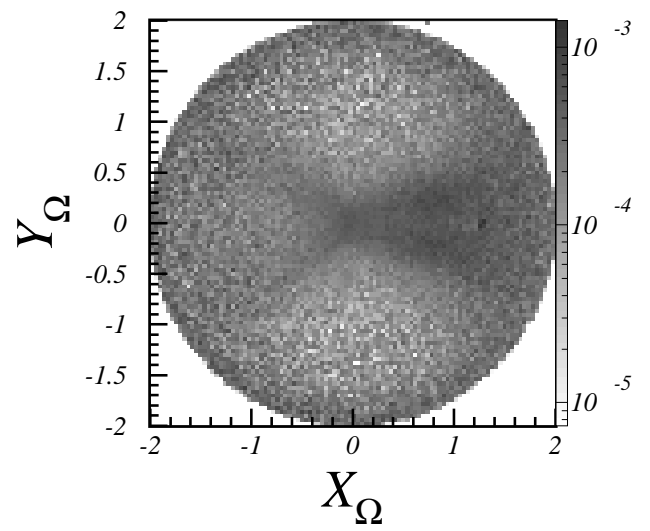

Figure 7.29: $X_{\Omega}$ vs. $Y_{\Omega}$ with radiative cut and $W>1.1 \mathrm{GeV}$ applied.

of Fig. 7.8 look quite clean, no further simulation or subtraction are needed in the current work. 


\section{CHAPTER 8 \\ Observable Extraction}

\subsection{Observables from Experiments}

Once one has a concrete description of the observables which are to be extracted from a data set, it is then necessary to study the logistics of such an extraction. Though these studies deal primarily with the practical challenges which an experiment presents it is not correct to consider these sorts of studies as pure logistics. It is often required to have a substantial understanding of the physics being studied both from a kinematical and dynamical perspective. An obvious example of such a situation is the understanding of radiative effects in electron scattering experiments. In principle this sort of physics can be loosely referred to as a "background" in that it is not of interest from an experimental point of view. It was realized in previous chapters that radiative effects and a host of other types of physics cannot be separated from measurement even in principle. It is just these types of situations which requires one to understand the mechanics of the extraction of observables from detectors. The present chapter will concern itself with precisely this area of understanding, while trying to stay as general as possible. It is possible, however, to gain insight through an analysis of what quantities are actually measured and how they are related to the quantities discussed in the previous chapters.

\subsection{Invariants in the Lab}

The first challenge in an experiment is identifying physical kinematics and gaining insight on the phase space geometry which these kinematics generate. Indeed, a rough estimate along these lines is necessary in order to check the feasibility of a measurement with a particular apparatus.

When one examines electron scattering off of a stationary target in the process $e+p \rightarrow e^{\prime}+p^{\prime}+M^{1}$ it is first, useful to understand the kinematics of the electron current. Since the target will be hadronic in any reasonable experiment, it is clear

\footnotetext{
${ }^{1} \mathrm{M}$ is a meson in the final state.
} 
that there will necessarily be an electron in the final state. Normally, a hadronic target is probed by a t-channel electromagnetic exchange so that the momentum and energy transfered to the target is given simply by the initial and final state electron momenta. Assuming the 4-momentum transfer from the electron current is $q$ one can simply relate the magnitude of this 4-vector to laboratory observables.

$$
Q^{2}=-q^{2}=2 E E^{\prime}\left(1-\cos \theta_{e}\right)
$$

In the laboratory frame $\theta_{e}$ is the angle between the outgoing electron and the beam, $E^{\prime}$ is its energy and $E$ is the incoming electron energy. The other invariant which completely specifies the electron kinematics is the invariant energy $W$. This can be written in the lab frame quite simply.

$$
W=\sqrt{(q+p)^{2}}=\sqrt{m_{p}^{2}+2 q^{0} m_{p}-Q^{2}}
$$

The most useful way to summarize this information is to observe how different values for the invariants $Q^{2}$ and $W$ map on to the more practical laboratory observables such as $E^{\prime}$ and $\theta_{e}$. Figure 8.1 shows the laboratory variables for several $W$ contours. The electron momenta are referred to as $k$ and $k^{\prime}$. Each of the curves is parameterized by $Q^{2}$ which runs from 5.0 to $9.0 \mathrm{GeV}^{2}$. The incoming electron energy is $E=5.5 \mathrm{GeV}$. Also pictured in Fig. 8.1 is the geometric acceptance for two electron arm spectrometer settings in the experiment ${ }^{2}$ at the Thomas Jefferson National Accelerator Facility (TJNAF) Hall C. At a given $W$ one can see that there are two $Q^{2}$ ranges which are gathered with the two settings.

It is also useful to understand the kinematics of the hadronic part of the interaction which is assumed to be comprised of a target (nucleon) and an extra final state meson. Since for simple electroproduction one has three final state particles, the reaction need not be in plane. This fact makes for a non-planar reaction but some useful knowledge can be gained by assuming the reaction happened in plane. Figure 8.2 displays this in-plane process which will give useful limits on the hadronic angle $\theta_{q p}$. For a given $W$ and detected hadronic momentum $\left|\mathbf{p}^{\prime}\right|$ the planar schematic

\footnotetext{
${ }^{2}$ The experiment is e01-002 and is funded by the DOE.
} 


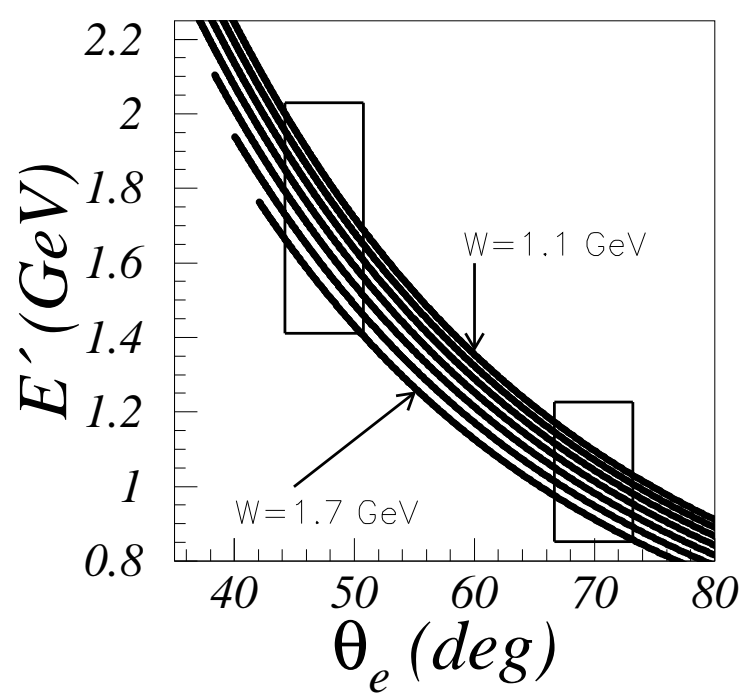

Figure 8.1: Lab frame quantities for $W$ contours in $0.1 \mathrm{GeV}$ step increments running from $Q^{2}$ of $5.0 \mathrm{GeV}^{2}$ to $9.0 \mathrm{GeV}^{2}$ for $E=5.5$ GeV.

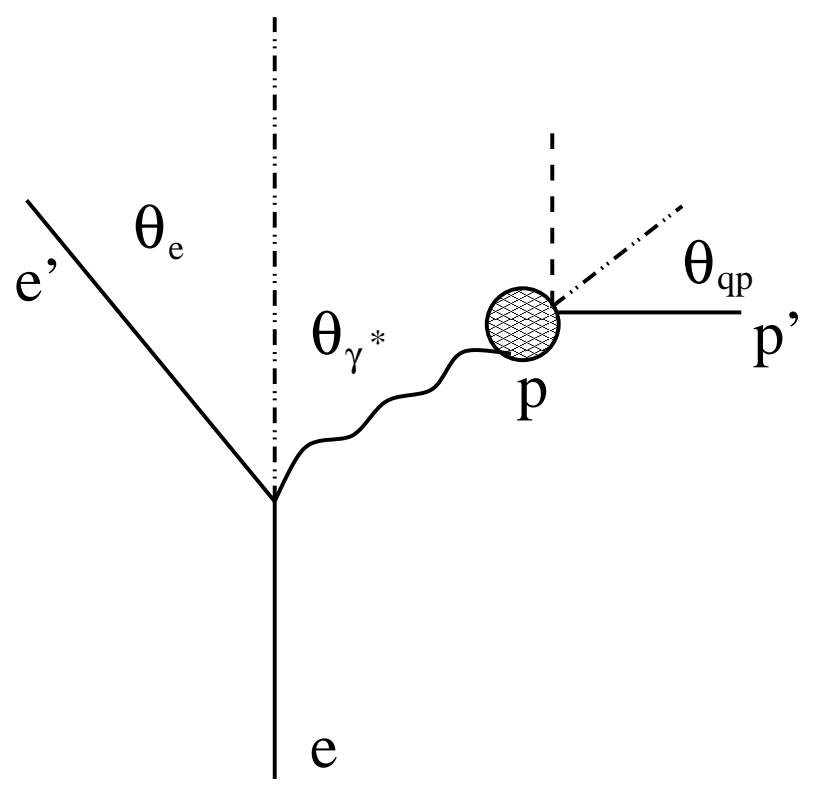

Figure 8.2: Lab frame hadronic kinematics.

in Fig. 8.2 yields the maximum angle $\theta_{q p}$ which can be used to obtain the maximum laboratory angle for the outgoing nucleon $\theta_{\gamma^{*}}+\theta_{q p}$. In order to solve for these angles and momenta one can use simple energy and momentum conservation. If one assumes that the outgoing meson has the mass $m_{m}$ then energy conservation can be 
written as thus.

$$
q^{0}+p^{0}=\sqrt{m_{m}^{2}+\left|\mathbf{k}_{m}\right|^{2}}+\sqrt{m_{p}^{2}+\left|\mathbf{p}^{\prime}\right|^{2}}
$$

Momentum conservation yields similar equations which will allow one to solve for the nucleon momentum $\left|\mathbf{p}^{\prime}\right|$ and the relative angle $\theta_{q p}$.

$$
\begin{aligned}
|\mathbf{q}| & =\left|\mathbf{p}^{\prime}\right| \cos \theta_{q p}+\left|\mathbf{k}_{m}\right| \cos \psi \\
\left|\mathbf{p}^{\prime}\right| \sin \theta_{q p} & =\left|\mathbf{k}_{m}\right| \sin \psi
\end{aligned}
$$

The angle $\psi$ is the laboratory angle between the momentum transfer $\mathbf{q}$ and the outgoing meson momentum $\mathbf{k}_{m}$. One can write these kinematics in the laboratory frame and then use the results in order to observe how the lab frame variables relate to the kinematic invariants which are useful for parameterizing physical dynamics.

$$
\begin{aligned}
q^{0}+p^{0} & =\left(\frac{W^{2}+Q^{2}+m_{p}^{2}}{2 m_{p}}\right) \\
|\mathbf{q}| & =\sqrt{\left(\frac{W^{2}+Q^{2}-m_{p}^{2}}{2 m_{p}}\right)^{2}+Q^{2}}
\end{aligned}
$$

Given the above equations one can construct a simple relation between the nucleon angle and momentum given a value of $Q^{2}$ and $W$.

$$
\left|\mathbf{k}_{m}\right|^{2}=|\mathbf{q}|^{2}-2|\mathbf{q}|\left|\mathbf{p}^{\prime}\right| \cos \theta_{q p}+\left|\mathbf{p}^{\prime}\right|^{2}
$$

This can be written in terms of the kinematical quantities worked out in Eqn. 8.5. Finally the cosine of the angle of the outgoing nucleon relative to the momentum transfer vector can be related to the outgoing nucleon momentum.

$$
\cos \theta_{q p}=\frac{\left(\left(q^{0}+p^{0}-\sqrt{m_{p}^{2}+\left|\mathbf{p}^{\prime}\right|^{2}}\right)^{2}-m_{m}^{2}-\left|\mathbf{p}^{\prime}\right|^{2}-|\mathbf{q}|^{2}\right)}{2|\mathbf{q}|\left|\mathbf{p}^{\prime}\right|}
$$

Figure 8.3 displays the contours for constant $W$ for $.1 \mathrm{GeV}$ intervals beginning from $W=1.1 \mathrm{GeV}$ and ending at $W=1.4 \mathrm{GeV}$. The $Q^{2}$ for each of these contours is selected based on which value puts the outgoing electron closest to the center of 
the geometrical acceptance pictured in Fig. 8.1 centered about $47.5^{\circ}$. Each of the

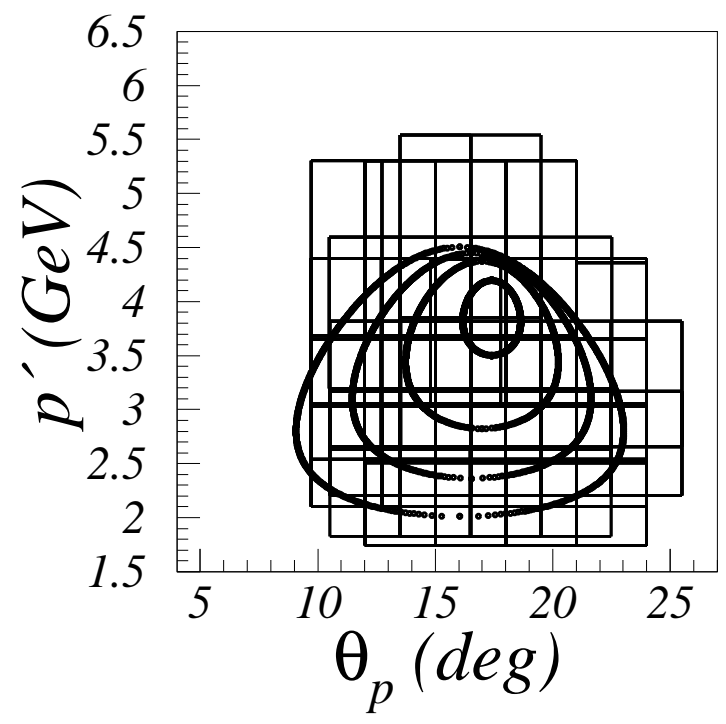

Figure 8.3: $W$ contours for planar kinematics in the laboratory variables for the outgoing nucleon. The contours are calculated for $\theta_{e}=47.5^{\circ}$ and the boxes represent the HMS settings from this experiment.

rectangular areas in the background of Fig. 8.3 represent the geometric acceptances for hadron spectrometer settings in the Hall $\mathrm{C}$ experiment similar to the geometric acceptances displayed in Fig. 8.1. The hadronic kinematics for the higher invariant momentum transfer setting can similarly be obtained. The values of $Q^{2}$ in Fig. 8.4 are thus selected to be closest to the center of the geometrical acceptance pictured in Fig. 8.1 centered about $69.9^{\circ}$. Figure 8.4 , once again, shows $W$ contours which begin from $1.1 \mathrm{GeV}$ and proceed in $0.1 \mathrm{GeV}$ steps to $W=1.4 \mathrm{GeV}$. The innermost contour is $1.1 \mathrm{GeV}$ and proceeds outward. The kinematics in Fig. 8.4 are covered by experimental settings such that all $W$ are present but the full angular kinematics may not be represented. All the kinematics in this figure are related to higher $Q^{2}$ than in Fig. 8.3.

It should also be noted that within each contour figure the value of $Q^{2}$ being probed by the spectrometer will change slightly with increasing $W$. The effect actually makes the data represent lower $Q^{2}$ values with increasing $W$. This effect is easy to understand in view of Fig. 8.1. The $Q^{2}$ values that correspond to the 


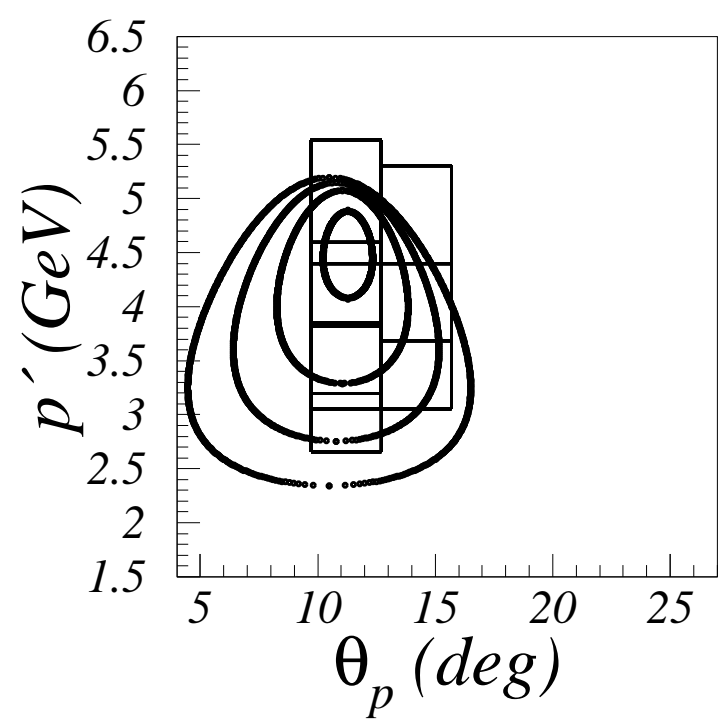

Figure 8.4: $W$ contours for planar kinematics in the laboratory variables for the outgoing nucleon. The contours are calculated for $\theta_{e}=69.9^{\circ}$ and the boxes represent the HMS settings from this experiment.

center (or closest to) of the spectrometer can be easily calculated. The true values

\begin{tabular}{|c|c|c|c|}
\hline SOS $\measuredangle$ & SOS $p(\mathrm{GeV})$ & W $(\mathrm{GeV})$ & $Q^{2}\left(\mathrm{GeV}^{2}\right)$ \\
\hline $47.5^{\circ}$ & 1.72 & 1.1 & 6.59 \\
\hline $47.5^{\circ}$ & 1.72 & 1.2 & 6.42 \\
\hline $47.5^{\circ}$ & 1.72 & 1.232 & 6.36 \\
\hline $47.5^{\circ}$ & 1.72 & 1.3 & 6.24 \\
\hline $47.5^{\circ}$ & 1.72 & 1.4 & 6.05 \\
\hline $69.9^{\circ}$ & 1.04 & 1.1 & 7.94 \\
\hline $69.9^{\circ}$ & 1.04 & 1.2 & 7.75 \\
\hline $69.9^{\circ}$ & 1.04 & 1.232 & 7.69 \\
\hline $69.9^{\circ}$ & 1.04 & 1.3 & 7.55 \\
\hline $69.9^{\circ}$ & 1.04 & 1.4 & 7.33 \\
\hline
\end{tabular}

Table 8.1: $Q^{2}$ values for each invariant energy bin. Invariant energy and $Q^{2}$ are not independent but are kinematically correlated.

of $Q^{2}$ listed in Tbl. 8.1 should be accounted for in actual data measurements. If one wishes to report data at a single value of $Q^{2}$ a correction factor is necessary. This means that some form of $Q^{2}$ has to be assumed or fit in order to make plots at a consistent $Q^{2}$. For the raw data as it applies to the pool of world data on the 
subject, the $Q^{2}$ values for each data point can simply be quoted as the measured value as the rescaling is not necessary for a global fit.

\subsection{Detector Acceptance}

One of the most important aspects of detectors which resolve various kinematic variables, especially angular ones, is the detectors acceptance. Clearly if particles (perhaps from a simple sample decay) emerge isotropically and one only detects the "northern hemisphere" of the angular coverage, the number of counts will be half what is expected for a given decay rate. This situation is summarized by saying that the geometrical acceptance is $50 \%$. In this case the acceptance was strictly geometrical and can be summarized by noting exactly where the detectors are as was seen in Figs. 8.1 ,8.3, and 8.4. The general treatment of acceptance can be more complicated because it is not necessarily true that the detector has a uniform efficiency at each point in its geometrical acceptance.

\subsubsection{General Acceptance}

Actual particle detectors cannot exhibit exact rotational symmetry. Indeed, the principle of detection for some detector systems prevent such a symmetry ${ }^{3}$. The consequence of this inherent lack of symmetry, whether it be due to designed asymmetry or random hardware differences of the detector components is that measurements taken in different regions of the detector to occur with different "efficiencies." This effect of a spatially varying efficiency is usually referred to as detector "acceptance" and includes the previously mentioned geometrical acceptance. One of the most difficult aspects of such an effect is that measurements are often concerned with kinematic variables that depend on the "natural" coordinates of the process as discussed in Chapter 2 but the acceptance is obviously most easily characterized using spacial coordinates with an origin at a convenient place in the lab.

For particle detectors a natural place to start characterizing the acceptance is writing down how many particles one expects to see in a kinematic bin. Though we have a discrete number of particles it is useful to consider the observed number

\footnotetext{
${ }^{3}$ For example, spectrometer detectors make use of a spacial asymmetry in the dispersive direction to make measurements.
} 
as coming from the sampling of a distribution. In that light, the following intuitive equation makes sense.

$$
d N=\mathcal{L} \epsilon_{d} d(A \sigma)
$$

The symbol $d N$ is to denote a measure for particle number that when integrated over the entire physical domain yields the total number of scattered particles. The symbols $\mathcal{L}$ and $\epsilon_{d}$ stand for luminosity and detector efficiency respectively. Finally, the $d(A \sigma)$ stands for the measure induced by the combination of the physical cross section and acceptance. Obviously, can have no handle on the abstract meaning of $d N$ or $d(A \sigma)$ therefore we write these as weights on the measures induced by the appropriate kinematic space. Suppose that the variable $\Lambda$ is taken to refer to all of kinematic space (which in the case of meson electroproduction is a five dimensional phase space). One can then write the more understandable form of Eqn. 8.8.

$$
\left(\frac{d N}{d \Lambda}\right) d \Lambda=\mathcal{L} \epsilon_{d} A(\Lambda)\left(\frac{d \sigma}{d \Lambda}\right) d \Lambda
$$

The function $A(\Lambda)$ is known as the acceptance function and in general depends on the experimental configuration. The acceptance function provides a kinematic variable dependent efficiency as was previously discussed. A large part of the effort in an experimental analysis goes into correcting for this function in order to extract the physical differential cross section $\frac{d \sigma}{d \Lambda}$, or at least some approximation to it.

Take for example, the number of counts registered in a particular kinematic range denoted $\delta \Lambda_{i}$, with center $\Lambda_{i}$. The predicted number based on the cross section can be obtained straight away from Eqn. 8.9.

$$
N_{\delta \Lambda_{i}}=\int_{\delta \Lambda_{i}}\left(\frac{d N}{d \Lambda}\right) d \Lambda=\int_{\delta \Lambda_{i}} \mathcal{L} \epsilon_{d} A(\Lambda)\left(\frac{d \sigma}{d \Lambda}\right) d \Lambda
$$

If physical cross section does not vary much over the phase space region $\delta \Lambda_{i}$ one can make an approximation for the right hand side of Eqn. 8.10. This approximation should be good for any well behaved functions as the phase space volume $\delta \Lambda_{i}$ 
becomes small compared with the natural scale of the function.

$$
\left.N_{\delta \Lambda_{i}} \simeq\left[\mathcal{L} \epsilon_{d} \int_{\delta \Lambda_{i}} A(\Lambda) d \Lambda\right]\left(\frac{d \sigma}{d \Lambda}\right)\right|_{\Lambda_{i}}
$$

This is the basis for all cross section measurements. One now has a reasonable way to characterize the differential cross section ${ }^{4}$ via the measurement of a number of counts in a kinematic bin $\delta \Lambda_{i}$. As advertised, if one is armed with the measured number of counts and a knowledge of the luminosity ${ }^{5}$ the only additional piece of information that is needed to perform the extraction is some characterization of the detector acceptance function.

\subsubsection{Moving Spectrometer Acceptance}

It has been explained how the detector acceptance presents itself in an experiment with a single detector configuration and therefore a single acceptance function $A(\Lambda)$. The situation is complicated if one wants to sum together results from the same kinematic bin but possibly from many different detector configurations. The most obvious method for dealing with this is to analyze each detector configuration independently and obtain the full results. After each set of results is obtained they can be statistically combined. This method is good in principle but has a drawback for the present analysis. One of the particle identification cuts mentioned in the previous chapter, Chapter 7, depends on having a large enough sample of data to judiciously choose how to make the rejection. This was the missing mass restriction. Of course, one could join the data for the purposes of making that restriction and then separate it for the analysis but it is preferred to use a consistent method to avoid systematic errors.

One is then left with the question of how to deal with the acceptance over many configuration changes (as a spectrometer experiment will have in general). A good place to begin is to write the generalization of Eqn. 8.10 from the previous

\footnotetext{
${ }^{4}$ Which is a distribution.

${ }^{5}$ By luminosity here we mean charge per unit area. Rate is irrelevant as both the number of counts and the beam current are to be integrated over the running time of the experiment.
} 
section.

$$
N_{\delta \Lambda_{i}}=\sum_{j} N_{\delta \Lambda_{i}}^{j}=\sum_{j} \int_{\delta \Lambda_{i}} \mathcal{L}^{j} \epsilon_{d} A_{j}(\Lambda)\left(\frac{d \sigma}{d \Lambda}\right) d \Lambda
$$

In Eqn. $8.12 j$ indexes the detector configurations. Note that the acceptance function and luminosity can depend on detector configuration but the physical differential cross section obviously may not. Since the detector efficiency and luminosity are known it is useful to scale them out prior to executing the sum in Eqn. 8.12 by defining $\tilde{N}^{j} \equiv \frac{N^{j}}{\epsilon_{d} \mathcal{L}^{j}}$.

$$
\tilde{N}_{\delta \Lambda_{i}}=\sum_{j} \tilde{N}_{\delta \Lambda_{i}}^{j}=\sum_{j} \int_{\delta \Lambda_{i}} A_{j}(\Lambda)\left(\frac{d \sigma}{d \Lambda}\right) d \Lambda
$$

The sum obviously can move into the integral, and a simplification similar to Eqn. 8.11 of the previous section can be made.

$$
\left.\tilde{N}_{\delta \Lambda_{i}} \simeq\left[\int_{\delta \Lambda_{i}} \sum_{j} A_{j}(\Lambda) d \Lambda\right]\left(\frac{d \sigma}{d \Lambda}\right)\right|_{\Lambda_{i}}
$$

It is clear, then, that expression 8.14 provides a way to measure the differential cross section given information about the integrals of the sums of the acceptance functions over settings. Indeed these acceptance functions are difficult to calculate analytically, so that the previous characterization of measured data is not useful unless one has a reliable way to calculate (or otherwise cancel) the acceptance. Such a method will be realized via simulation technique.

\subsection{Monte Carlo Simulation}

The work of the previous sections makes the goals of a simulation procedure clear. We need the acceptance as it relates to the sum over all of the detector configurations.

There are generally two ways to proceed in constructing a simulation of a particle physics experiment. Either one can generate uniformly over a kinematical phase space and then weight by a distribution function (model cross section) including appropriate normalization factors, or one can attempt to generate data which has 
the appropriate distribution from the start. It is more straightforward to generate uniformly and then apply a simple numerical weight, this is the method advocated in this work (for the most part). Attempting to generate with the desired distribution from the start can help if the physical distribution is sharply peaked in one region of phase space so as to make a uniform generation inefficient in producing useful events. A good example of a process where it is important to try to generate events from the physical distribution is the elastic radiative process because of the strong affinity for radiated photons to emerge along the directions of either the incoming or outgoing electron currents.

There are many well-known methods for generating uniformly over some generalized phase space $\Delta \Lambda$ and then weighting events by a model cross section [95]. This ability coupled with a detailed model of the detection system including the resolutions in each kinematic variable will allow one to simulate an experiment properly. As the simulation moves on events will be rejected or accepted based on the properties of the detector system. Therefore, the number of accepted events in a particular kinematic bin can be written out in a similar fashion as to what has been seen in the previous sections.

$$
\begin{aligned}
N_{\delta \Lambda_{i}}^{m c} & =\int_{\delta \Lambda_{i}} \mathcal{L}^{m c} A^{m c}(\Lambda)\left(\frac{d \sigma^{m c}}{d \Lambda}\right) d \Lambda \\
N_{\delta \Lambda_{i}}^{m c} & \left.\simeq\left[\mathcal{L}^{m c} \int_{\delta \Lambda_{i}} A^{m c}(\Lambda) d \Lambda\right]\left(\frac{d \sigma^{m c}}{d \Lambda}\right)\right|_{\Lambda_{i}}
\end{aligned}
$$

The symbol $A^{m c}(\Lambda)$ is the acceptance function induced by the Monte Carlo acceptance and rejection in the detector model. The detector simulation is designed so that this acceptance function matches the physical acceptance as well as possible. These equations can be generalized to work with several detector configurations (again indexed by $j$ ) summed together.

$$
\left.N_{\delta \Lambda_{i}}^{m c} \simeq\left[\mathcal{L}^{m c} \int_{\delta \Lambda_{i}} \sum_{j} A_{j}^{m c}(\Lambda) d \Lambda\right]\left(\frac{d \sigma^{m c}}{d \Lambda}\right)\right|_{\Lambda_{i}}
$$


The luminosity can obviously be scaled out as with the data.

$$
\left.\tilde{N}_{\delta \Lambda_{i}}^{m c} \simeq\left[\int_{\delta \Lambda_{i}} \sum_{j} A_{j}^{m c}(\Lambda) d \Lambda\right]\left(\frac{d \sigma^{m c}}{d \Lambda}\right)\right|_{\Lambda_{i}}
$$

The quantity that one should form is then obvious, assuming $j$ runs over the same number of settings for both the data and the simulation.

$$
r_{\Lambda_{i}} \equiv \frac{\tilde{N}_{\delta \Lambda_{i}}}{\tilde{N}_{\delta \Lambda_{i}}^{m c}}
$$

To the extent that the acceptances are properly modeled we have that $A_{j}(\Lambda) \sim$ $A_{j}^{m c}(\Lambda)$. And so the above ratio has a simple interpretation in terms of the data and model differential cross sections.

$$
\left.r_{\Lambda_{i}} \simeq\left(\frac{d \sigma}{d \Lambda}\right)\right|_{\Lambda_{i}} /\left.\left(\frac{d \sigma^{m c}}{d \Lambda}\right)\right|_{\Lambda_{i}}
$$

This ratio is useful in extracting the data in a way that is to good approximation insensitive to the model cross section used.

\subsection{Cross Section Extraction}

Sections 8.3.2 and 8.4 covered how detectors with finite acceptance and experiments with many configuration changes ${ }^{6}$ can be treated in a consistent manner. By a method described in those sections one can measure the ratio of a cross section to a specified model cross section used to generate the acceptance model.

For the cross section extraction of the neutral pion production cross section from the current data, this method can be utilized. The simulation package used for both the signal process and elastic radiative background processes (where necessary) is the Hall C simulation package SIMC. This package was developed by many members of the Hall $\mathrm{C}$ collaboration and has been tuned to the appropriate magnet optics and efficiencies of the HMS and SOS spectrometers [7].

\footnotetext{
${ }^{6}$ This means the acceptance can change discontinuously depending on process independent measurement details such as detector setting.
} 
The analysis of the previous sections was not marred by background and efficiency considerations. Here all the logistics of the cross section extraction will be considered as they pertain to the experiment at hand. Firstly, the count numbers referred to in the previous sections were idealized and represented only the signal process. The number of counts in a kinematic bin can be easily represented as the sum of signal processes and background processes. Indexing the kinematic bins by $i$ instead of the $\Lambda_{i}$ of the previous sections, we have:

$$
N_{i}^{e x p}=N_{i}+N_{i}^{\gamma}+N_{i}^{a c c}+N_{i}^{t a r}
$$

The notation is such that $N^{e x p}$ is the number of counts observed in the experiment and is composed of $N_{i}$ which is the number from the signal process, $N_{i}^{\gamma}$ which is the number from Bethe-Heitler events, $N_{i}^{a c c}$ are the accidental counts, and $N_{i}^{\text {tar }}$ are events which emerge from the target container materials. One can immediately move to the scaled number of counts so long as one remembers that the Poisson statistics (assigning a standard deviation of $\sqrt{N}$ ) are to be applied only to the portion of the experiment which is actually observed and the efficiency scalings are to be propagated through. For each experimental configuration (indexed by $j$ ) one will have the scaled number of counts.

$$
\tilde{N}_{i j}^{e x p}=\frac{N_{i j}^{e x p}}{\mathcal{L}_{j} \epsilon_{j}}
$$

Where, $N_{i}^{e x p}=\sum_{j} N_{i j}^{e x p}$ and correspondingly for the scaled number of counts, similar to before. The possible efficiencies for the experiment are contained in the factor $\epsilon_{j}$. Generically, the efficiencies can be expanded as in Eqn. 8.22.

$$
\epsilon=\epsilon^{d c} \times \epsilon^{c d t} \times \epsilon^{e d t}
$$

If one is armed with a method to estimate the number of background counts or a way to exclude them in the first place, the following quantity can easily be formed.

$$
r_{i} \equiv \frac{\tilde{N}_{i}^{e x p}-\tilde{N}_{i}^{\gamma}-\tilde{N}_{i}^{a c c}-\tilde{N}^{t a r}}{\tilde{N}^{m c}}
$$


The ratio formed is equivalent to that observed in Eqn. 8.19 and can be used quite transparently to extract the signal process cross section.

$$
\left.\left(\frac{d \sigma}{d \Lambda}\right)\right|_{\Lambda_{i}}=\left.r_{i}\left(\frac{d \sigma^{m c}}{d \Lambda}\right)\right|_{\Lambda_{i}}
$$

The appropriate kinematic variables can be explicitly written.

$$
d \Lambda=d E^{\prime} d \Omega_{e} d \Omega_{\pi}^{*}
$$

Then the extraction equation becomes more explicit ${ }^{7}$.

$$
\left.\left(\frac{d \tilde{\sigma}}{d E^{\prime} d \Omega_{e} d \Omega_{\pi}^{*}}\right)\right|_{\Lambda_{i}}=\left.r_{i}\left(\frac{d \tilde{\sigma}^{m c}}{d E^{\prime} d \Omega_{e} d \Omega_{\pi}^{*}}\right)\right|_{\Lambda_{i}}
$$

Given the known form of the 5-fold differential cross section in terms of the virtual photon-proton center of mass cross section, one can go further in simplifying the previous expression.

$$
\frac{d \tilde{\sigma}}{d E^{\prime} d \Omega_{e} d \Omega_{\pi}^{*}}=\Gamma \frac{d \tilde{\sigma}}{d \Omega_{\pi}^{*}}
$$

The photon flux factor will cancel since it is the same in the numerator and denominator of Eqn. 8.26.

$$
\left.\left(\frac{d \tilde{\sigma}}{\Omega_{\pi}^{*}}\right)\right|_{\Lambda_{i}}=\left.r_{i}\left(\frac{d \tilde{\sigma}^{m c}}{\Omega_{\pi}^{*}}\right)\right|_{\Lambda_{i}}
$$

In the recent expressions $\Lambda_{i}$ still stands for the center bin position in all kinematic variables for kinematic bin $i$. Equation 8.28 embodies the method used to extract center of mass differential cross sections in this work. First we select a cross section in the center of mass to simulate with then we construct the appropriate ratio from the data analysis after all appropriate subtractions. After these two tasks it is a simple manipulation to produce a final extracted cross section.

In this section the extraction of the cross section which nature provides was covered. This means that we have paid no attention here to the fact that events

\footnotetext{
${ }^{7}$ Here we specialize to a specific cross section which will be denoted $d \tilde{\sigma}$. The reason for this is that this is not exactly the cross section which represents the pure single pion production, it lacks a radiative correction factor.
} 
with low energy radiated photons have necessarily included in the extraction. This means that the cross section extracted corresponds to that of Eqn. 4.9 while the desired cross section is the one represented by the first term on the right hand side of that equation only. This term can be extracted by correcting by a calculated radiative correction factor discussed in Section 8.7.

\subsection{Kinematic Bin Centering}

One more issue should be considered before the entire analysis procedure is documented. Though the cross section extraction strives to introduce as little error as possible into the mix, because of finite statistics one must work with kinematic bins which are large enough to have a reasonable amount of counts. It is possible that the true cross sections vary a significant amount over the bins. Reporting the raw extracted cross section at the bin centers (equidistant from each bin edge) may not be the most logical solution in these cases. This point is most easily illustrated by an example. Figure 8.5 shows the binning situation for a measured variable $y$ and independent variable $x$. In the previous sections it was assumed that the

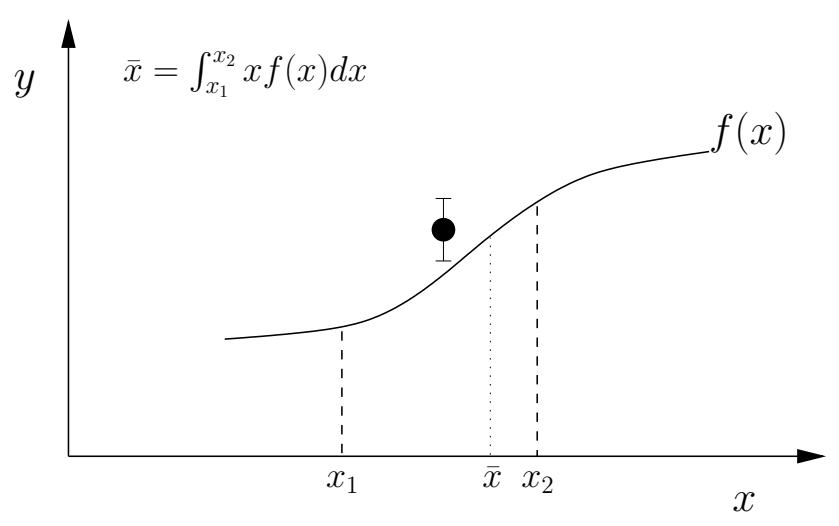

\section{Figure 8.5: Typical binning situation.}

cross section does not vary over the kinematic bin. The corrections applied here are meant to correct for that. There are basically two ways that one can deal with the apparent variation of the measured distribution, similar to the situation pictured in Fig. 8.5. One can either choose a new point to report the cross sections at, based on the curve for the dependence of the distribution or one can modify the value of 
the data point slightly. A logical place to report the cross section is at the centroid of the distribution within each bin. It is however, not desirable to modify the kinematic points at which data is being reported since they were originally laid out in a (presumably) regular fashion and this is the easiest format for interpretation. As a result, in this work the values will be modified by a correction factor for the finite bin sizes.

Notice that if the model is exactly what the data says it "should" be (i.e. from the result of a fit) or if the bin sizes go to zero then whatever bin centering factor that is chosen should go to unity. A reasonable bin centering factor $f$ to apply to the data can be based off of a fit cross section $\sigma_{f}(\Lambda)$, where $\Lambda$, again refers to all kinematic quantities. Keeping with the previously used notation one can define:

$$
f_{i} \equiv \frac{\int_{\Lambda_{i}} d \Lambda d \sigma^{m c}(\Lambda)}{\int_{\Lambda_{i}} d \Lambda d \sigma_{f}(\Lambda)} \frac{d \sigma_{f}\left(\Lambda_{R}\right)}{d \sigma^{m c}\left(\Lambda_{R}\right)}
$$

With the generic shorthand notation:

$$
d \sigma(\Lambda) \equiv\left(\frac{d \sigma}{d \Lambda}\right)
$$

The symbol $\Lambda_{R}$ stands for the value of kinematics one wants to report the experimentally measured cross sections at. Thus defined, these bin centering factors satisfy all the requisite conditions. If the bin sizes approach zero ${ }^{8}$, the first fractional factor in Eqn. 8.29 will approach $\frac{d \sigma^{m c}\left(\Lambda_{R}\right) \times \epsilon}{d \sigma_{f}\left(\Lambda_{R}\right) \times \epsilon}$, where $\epsilon$ is small. This is the inverse of the second fractional term in the limit $\epsilon \rightarrow 0$. On the other hand, if the fitted distribution exactly equals the simulated distribution, each fractional factor in the equation will be unity. This correction is thus a robust way to take into account the slight variation of the cross sections inside kinematic bins.

\subsection{Full Analysis Procedure}

Now that all the cards are on the table (except the issue of meson radiative corrections, which is addressed in what follows) it is useful to put these together in

\footnotetext{
${ }^{8}$ They should approach zero symmetrically about $\Lambda_{R}$.
} 
one section so that a clear logical progression is established and adhered to. This is most easily done with a list of steps.

1. Select binning scheme: Select an appropriate binning scheme in the kinematic variables $\left\{W, \cos \theta^{*}, \phi^{*}\right\}$. This selection may be changed when the statistics of each bin are previewed. The current experimental statistics suggest a reasonable binning for the data reported in Tbls. 8.2 and 8.3.

\begin{tabular}{|c|c|c|c|}
\hline variable & $W(\mathrm{GeV})$ & $\cos \theta^{*}$ & $\phi^{*}(\mathrm{rad})$ \\
\hline range & $1.092 \leq W \leq 1.412$ & $-1.0 \leq \cos \theta^{*} \leq 1.0$ & $-\pi \leq \phi^{*} \leq \pi$ \\
\hline bins & 8 & 10 & 10 \\
\hline
\end{tabular}

Table 8.2: E01-002 analysis binning for low $Q^{2}$ data.

\begin{tabular}{|c|c|c|c|}
\hline variable & $W(\mathrm{GeV})$ & $\cos \theta^{*}$ & $\phi^{*}(\mathrm{rad})$ \\
\hline range & $1.092 \leq W \leq 1.412$ & $-1.0 \leq \cos \theta^{*} \leq 1.0$ & $-\pi \leq \phi^{*} \leq \pi$ \\
\hline bins & 8 & 6 & 6 \\
\hline
\end{tabular}

Table 8.3: E01-002 analysis binning for high $Q^{2}$ data.

2. Apply data restrictions: Apply all the data restrictions listed in Chapter 7. This includes the missing mass restrictions, which are applied on a bin by bin basis.

3. Make bin by bin subtractions: Subtract accidentals or other contaminants by using the data set itself to estimate the contribution in each kinematic bin or by using a simulation technique.

4. Simulate data using model cross section: Put together a model cross section for neutral pion production and simulate to get the acceptance. In this work the initial model for the cross section was simply a flat distribution at $1 \mu \mathrm{b} / \mathrm{Sr}$ in the center of mass angles.

5. Use ratio to obtain physical cross section: The ratio method described in Section 8.4 is used to cancel the acceptance of the detection apparatus in 
places with non-zero acceptance. The acceptance function is never explicitly obtained (but can be).

6. Apply radiative corrections: This is the step that has been put off until now. Typically, for Hall $\mathrm{C}$ studies the radiative corrections are applied implicitly by putting them into the simulation package. In this method one is comparing radiated two radiated cross sections and the ratio of the number of counts is taken to be the same as the ratio of two born level (non-radiated) cross sections. Current codes which compute the radiative effects [2] are too computationally intensive to calculate the full radiative correction on the fly, so "peaking approximations" are used [36]. For exclusive processes this should not introduce large systematic errors but here we follow the more direct approach of extracting a physical cross section (with photon radiation included) and correcting this cross section. The cross section that is extracted has been displayed in Section 4.3 Eqn. 4.9 and is repeated here.

$$
\frac{d \sigma_{m}^{m e s}}{d \Omega_{e} d E^{\prime} d \Omega_{\pi}}=\frac{d \sigma^{m}}{d \Omega_{e} d E^{\prime} d \Omega_{\pi}}+\int_{0}^{\Delta E} \frac{d^{3} k}{(2 \pi)^{3} 2 E_{\gamma}} \frac{d \sigma^{m \gamma}}{d \Omega_{e} d E^{\prime} d \Omega_{\pi} d E_{\gamma} d \Omega_{\gamma}}
$$

The center of mass cross section extracted corresponds to physical cross section displayed on the left hand side of this equation. The corresponding center of mass cross section has been mentioned in previous sections, particularly Eqn. 8.27 and is repeated here in the current notation.

$$
\frac{d \sigma_{m}^{m e s}}{d E^{\prime} d \Omega_{e} d \Omega_{\pi}^{*}}=\Gamma \frac{d \tilde{\sigma}}{d \Omega_{\pi}^{*}}
$$

If one recalls Eqn. 4.12, the factor which one must apply to make $d \tilde{\sigma}$ into the final measured born-level cross section, $d \sigma^{\gamma^{*}}$ is simply $\frac{1}{\delta}$ where:

$$
\delta \equiv \frac{\sigma_{m}^{m e s}}{\sigma^{m}}
$$

is the EXCLURAD [2] calculated radiative correction. The code EXCLURAD must be supplied with a model and we use MAID03 [31] as the standard, 
extrapolating the response functions to higher $Q^{2}$ by a dipole factor.

$$
\frac{d \sigma^{\gamma^{*}}}{d \Omega_{\pi}^{*}}=\frac{1}{\delta} \frac{d \tilde{\sigma}}{d \Omega_{\pi}^{*}}
$$

The final extracted cross section is displayed in Eqn. 8.31. One might worry that this procedure is marred by subtle acceptance effects in the Monte Carlo simulation. If one relaxes the constraint that the model and "data" should look the same in distributions upon iteration, then this is not a problem. The acceptance functions which the Monte Carlo is used for should be the same for a given set of detected particles and their respective momenta. That is, the acceptance should not depend on what other particles are created in any given reaction. Therefore, the only possible problem which can, and will, arise in this procedure is that processes with different numbers of undetected particles can have non-zero cross sections in regions where processes with other undetected particles are kinematically forbidden. For example, elastic radiative events have a different phase space than the pure elastic events. However, one will never seek to measure a cross section in a kinematically dis-allowed region so the ratios will never be extracted in those troublesome regions. The only constraint, then, is that the simulated process has the same detected particles and is kinematically allowed in every region where one wishes to obtain the final cross section.

One should certainly get an idea of the size of the radiative corrections which are produced by the procedure. Figures 8.6 and 8.7 display the sizes of the correction factors in the region of the $\Delta$ resonance. The radiative correction is thus $20.0-25.0 \%$ in the $\Delta$ region for the nominal inelasticity values. Over all of the invariant energy the correction varies over the somewhat larger range 15.0-27.0\%. Since a change in the inelasticity parameter used in the analysis will change the radiative correction, a systematic error should be assigned. In this case the corrections for the nominal inelasticity parameters vary very little with a reasonable sized change in the inelasticity parameter (missing mass restriction). A sysetmatic error of $2.0 \%$ is used to be conservative (this 


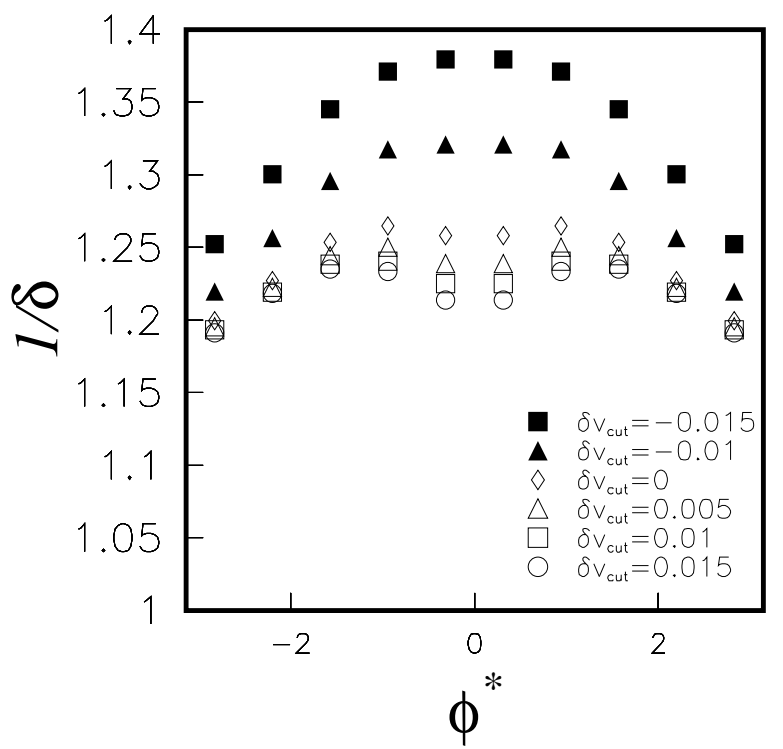

Figure 8.6: Radiative corrections for $W=1.232 \mathbf{G e V}$ and $Q^{2}=6.36 \mathbf{G e V}^{2}$. The inelasticity parameter $v$ was varied to produce several curves. The shift of the inelasticity parameter from nominal, $\delta v_{c u t}$ is displayed in the figure. The value of $\cos \theta^{*}$ is $\mathbf{0 . 0}$ here.

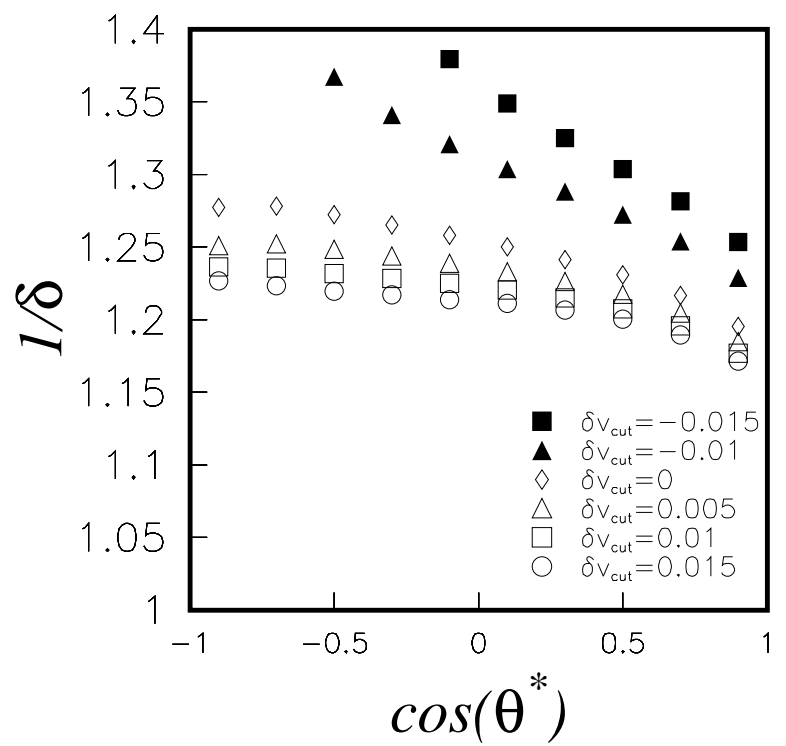

Figure 8.7: Radiative corrections for $W=1.232 \mathrm{GeV}$ and $Q^{2}=6.36 \mathbf{G e V}^{2}$. The inelasticity parameter $v$ was varied to produce several curves. The shift of the inelasticity parameter from nominal, $\delta v_{c u t}$ is displayed in the figure. The value of $\phi^{*}$ is $\mathbf{0 . 0}$ here. 
is the largest deviation).

7. Bin centering corrections: Apply the bin centering corrections to the new radiatively corrected cross section. This cross section can then be fit into any curve which approximates the shape well and fed back into the simulation. The procedure can be restarted from the Monte Carlo simulation, with hopes of convergence. This iteration process is displayed in Fig. 8.8. 


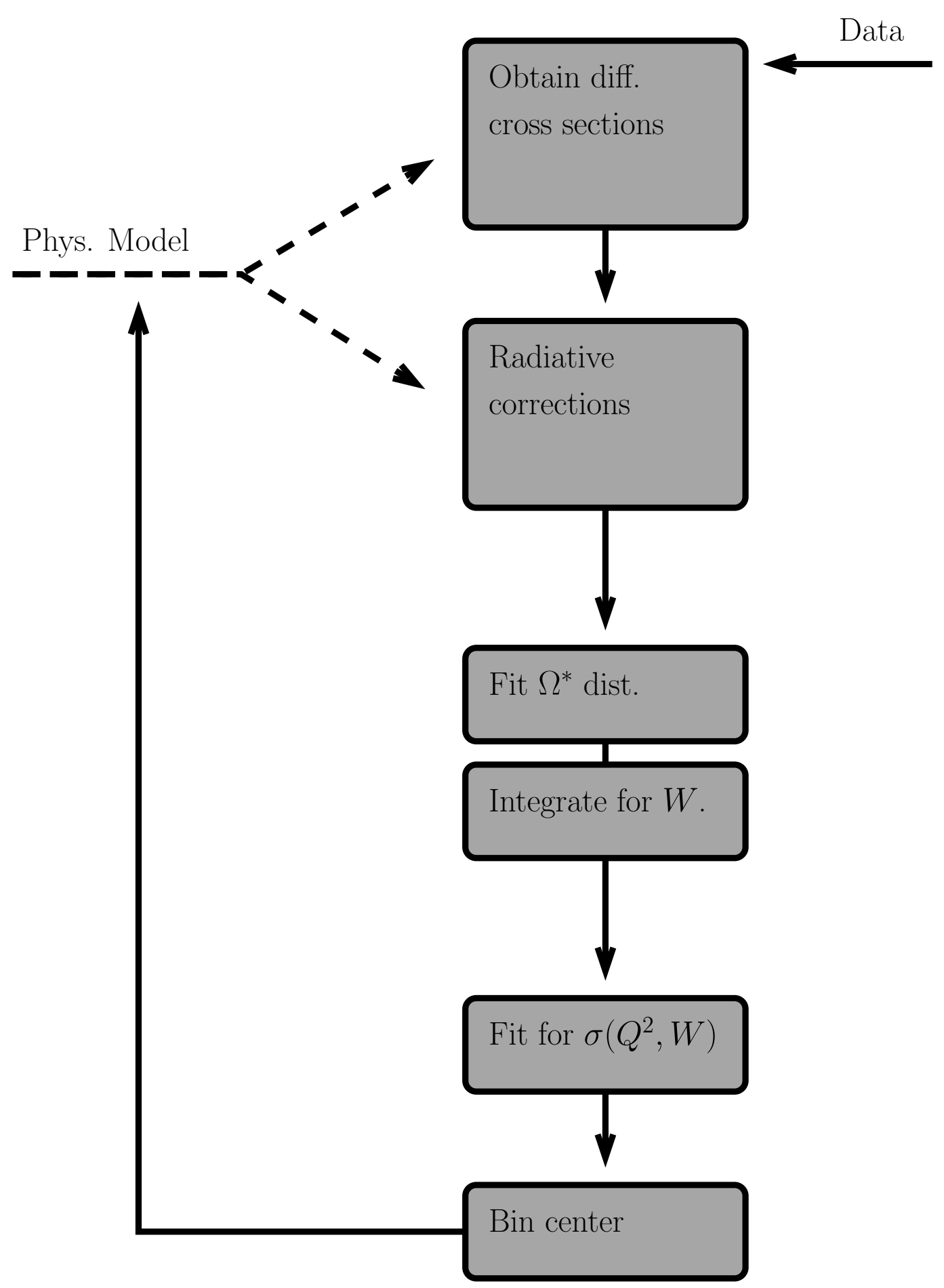

Figure 8.8: Flow chart describing analysis procedure. 


\section{CHAPTER 9 \\ Consistency Checks for Cross Sections}

\subsection{Data Checks}

To ensure the integrity of any extracted data set one should perform consistency checks. Unfortunately, one often has to look hard for ways to test the validity of extracted parameters since it is unlikely that copious amounts of data gathered independently exist for a new result. One then turns to agreement with previous approximate models, models of different processes which are related to the signal process, and agreement of a data set with itself (self-consistency). There are two primary consistency checks which can be applied to the data for single neutral pion production gathered from the E01-002 experiment. Firstly, since the present analyzed data includes processes below $\eta$ production threshold one can examine the inclusive cross section compared to the cross section obtained with only $\pi^{0}$ exclusive production data. In the region of invariant mass that is present in this experiment the inclusive cross section is the sum of only three processes of which $\pi^{0}$ production is one. The other consistency check relates to how the experiment E01-002 was performed. As it turns out for a certain region of electron detection kinematics there are two independent sets of experimental settings which could in principle cover the entire angular acceptance on their own and be used to obtain the same results but with slightly larger statistical errors. That these two data sets agree is an important self consistency check and will ensure that there are no important effects coming from setting overlaps or a misunderstanding of the spectrometer acceptances.

\subsection{Inclusive Check}

The integrated cross section for the center of mass $\pi^{0}$ reaction can be represented easily in terms of the electron angular variables so as to restore the original 5 -fold differential cross section of $e p \rightarrow e^{\prime} p^{\prime} \pi^{0}$. The kinematic factor necessary is the 
photon flux factor, $\Gamma$, as discussed in Section 2.2.

$$
\frac{d \sigma}{d E^{\prime} d \Omega_{e} d \Omega_{\pi}}=\Gamma \frac{d \sigma^{\gamma^{*}}}{d \Omega_{\pi}}
$$

Since the laboratory frame and the center of mass frame discussed in Section 2.2 are related by a boost parallel to both the incident virtual photon $\left(\gamma^{*}\right)$ beam and the incident proton, the differential cross section behaves like a scalar. The integration of the pion production cross section can then be performed in the center of mass, and applied to Eqn. 9.1. Written in the center of mass, the pion production cross section has the previously discussed explicit form for an unpolarized reaction [29].

$$
\begin{aligned}
\frac{d \sigma^{\gamma^{*}}}{d \Omega_{\pi}^{*}}=\frac{1}{2 \pi}\left[\frac{d \sigma_{T}}{d \cos \theta^{*}}+\epsilon \frac{d \sigma_{L}}{d \cos \theta^{*}}+\epsilon \frac{d \sigma_{T T}}{d \cos \theta^{*}}\right. & \cos 2 \phi^{*} \\
& \left.+\sqrt{2 \epsilon(1+\epsilon)} \frac{d \sigma_{L T}}{d \cos \theta^{*}} \cos \phi^{*}\right]
\end{aligned}
$$

Because of the symmetric $\phi^{*}$ dependence of the unpolarized cross section, the last two terms of Eqn. 9.2 integrate to zero. Define the integrals of the first two terms as per the following equation.

$$
\begin{aligned}
\sigma_{T}^{\pi^{0}} & \equiv \int d \cos \theta^{*} \frac{d \sigma_{T}}{d \cos \theta^{*}} \\
\sigma_{L}^{\pi^{0}} & \equiv \int d \cos \theta^{*} \frac{d \sigma_{L}}{d \cos \theta^{*}}
\end{aligned}
$$

These manipulations make the integrated cross section, take the following form.

$$
\frac{d \sigma^{\pi^{0}}}{d E^{\prime} d \Omega_{e}}=\Gamma\left(\sigma_{T}^{\pi^{0}}+\epsilon \sigma_{L}^{\pi^{0}}\right)
$$

Where there could be confusion (where the cross section is not a differential in pion kinematics) the integrated $\pi^{0}$ cross sections are denoted as $\sigma^{\pi^{0}}$ whereas the inclusive versions will have no superscripts. One is now in a position to compare to a model for inclusive cross sections. Remembering back to Section 1.3 one can easily see how a model for the structure functions $F_{1}$ and $F_{2}$ or cross sections $\sigma_{T}$ and $\sigma_{L}$ will lead 
to a specification of the inclusive cross section.

$$
\frac{d \sigma_{i n}}{d E^{\prime} d \Omega_{e}}=\Gamma\left(\sigma_{T}+\epsilon \sigma_{L}\right)
$$

A model for the necessary cross sections is available and widely used in the Hall $\mathrm{C}$ collaboration. The model uses fits to recent Hall $\mathrm{C}$ inclusive data and obtains an acceptable parameterization for seven of the most prominent resonances in the first 4 resonance regions for inclusive scattering off of a proton target. Figure 9.1 shows the comparison of the model to the measured integrated $\pi^{0}$ cross section.

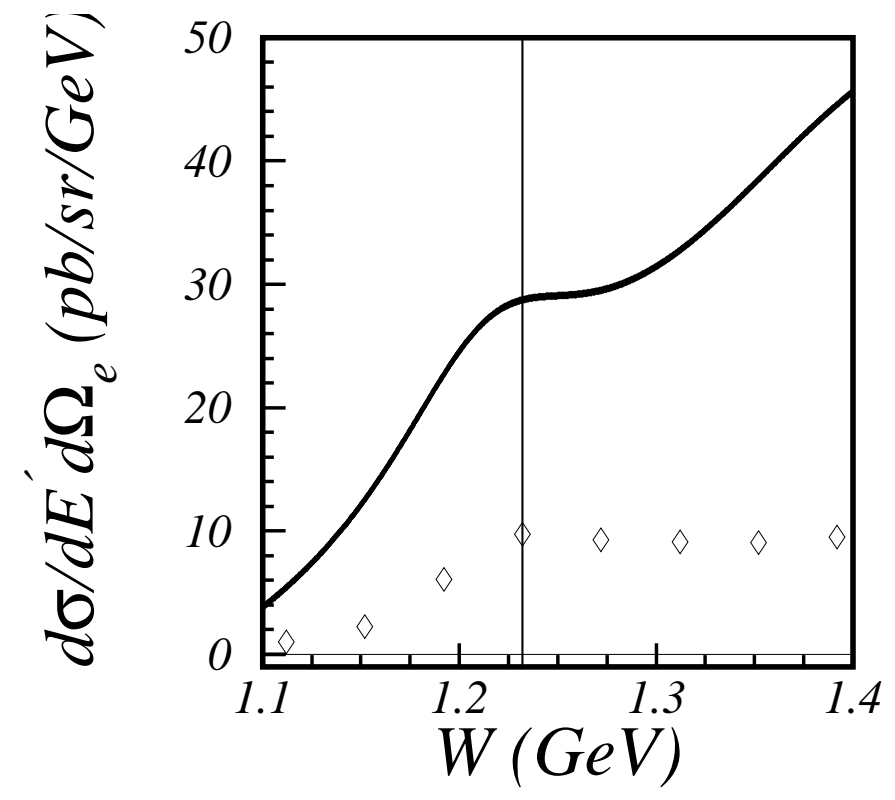

Figure 9.1: Comparison to inclusive cross section at $Q^{2}=6.36$.

Figure 9.2 shows the ratio of the integrated $\pi^{0}$ cross section to the inclusive model. It is notable that the data roughly tracks with the inclusive data but is strictly below it for $W$ below about $1.206 \mathrm{GeV}$ which is threshold for multiple pion production.

Because of the limited number of processes which can exist in this kinematic region, one can go a bit further in understanding this comparison. Since the dominant resonance the region $W$ near $1.232 \mathrm{GeV}$ is the $\Delta(1232)$, and this is a state of definite isospin $\left(I=\frac{3}{2} ; I_{3}=\frac{1}{2}\right)$ a decomposition can be made for $W_{\Delta}=1.232 \mathrm{GeV}$. The total inclusive cross section is made up of three components, the $I=\frac{3}{2}$ resonant component along with an $I=\frac{1}{2}$ nucleon component and a component whose 


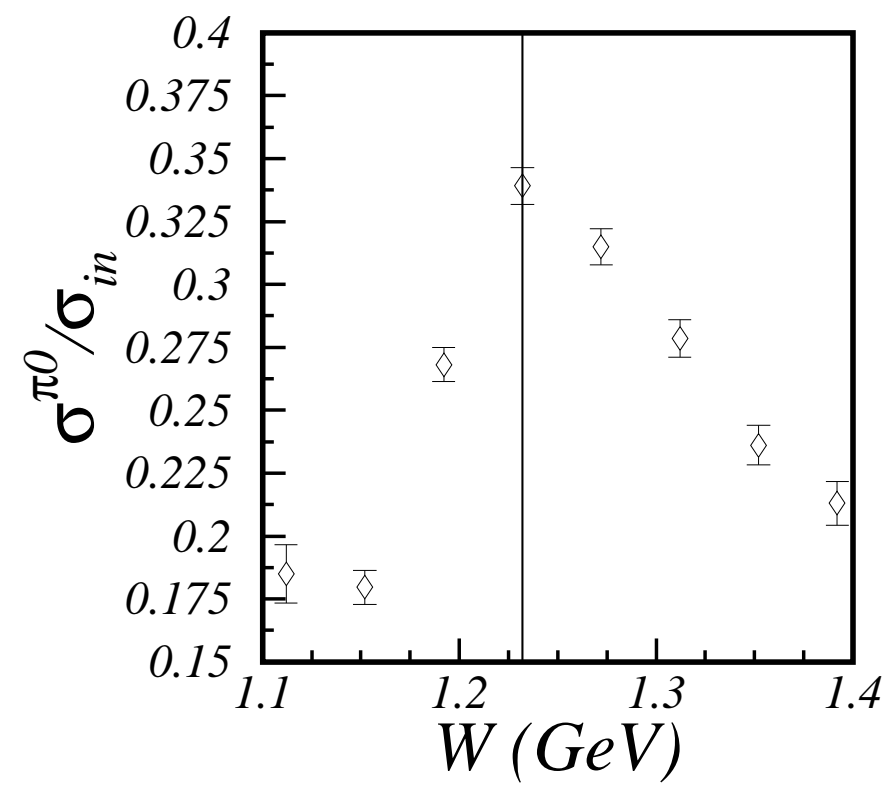

Figure 9.2: Ratio to inclusive cross section at $Q^{2}=6.36$.

intermediate state is not a definite isospin state (the subscript "m" will stand for $\left.\operatorname{mixed}^{1}\right)$.

$$
d \sigma_{i n}=d \sigma_{\frac{3}{2}}+d \sigma_{\frac{1}{2}}+d \sigma_{m}
$$

Near the $\Delta$ resonance region the $\sigma_{\frac{3}{2}}$ should dominate and can also be broken down into two pieces. One of these pieces represents the $\Delta$ being excited and then decaying to a $\pi^{0} p$ final state and the other represents the decay to a $\pi^{+} n$ final state. The cross section measured in this work represents only the former but the contributions are related by isospin symmetry. Denote isospin eigenstates as $\left|M ; I, I_{3}\right\rangle$ where $M$ is some designation of the particle multiplet or a combination of particle symbols. The symbols $I$ and $I_{3}$ designate the isospin. Because of isospin conservation one can write several useful relations.

$$
d \sigma_{\frac{3}{2}} \propto\left|\left\langle\pi N ; \frac{3}{2}, \frac{1}{2} \mid \Delta ; \frac{3}{2}, \frac{1}{2}\right\rangle\right|^{2}
$$

One can then write the final state in the coupled basis when examining the physical

\footnotetext{
${ }^{1}$ The term $d \sigma_{m}$ is understood to contain any possible interferences with the pure amplitudes as well.
} 
production processes.

$$
\begin{gathered}
\left|\pi^{0}\right\rangle \equiv|\pi ; 1,0\rangle \\
|p\rangle \equiv\left|N ; \frac{1}{2}, \frac{1}{2}\right\rangle
\end{gathered}
$$

The physical cross sections $d \sigma^{\pi^{0}}$ and $d \sigma^{\pi^{+}}$each have an isospin decomposition as well.

$$
\begin{aligned}
& d \sigma^{\pi^{0}}=d \sigma_{\frac{3}{2}}^{\pi^{0}}+d \sigma_{\frac{1}{2}}^{\pi^{0}}+d \sigma_{m}^{\pi^{0}} \\
& d \sigma^{\pi^{+}}=d \sigma_{\frac{3}{2}}^{\pi^{+}}+d \sigma_{\frac{1}{2}}^{\pi^{+}}+d \sigma_{m}^{\pi^{+}}
\end{aligned}
$$

One also has the physical cross sections as they are related to isospin matrix elements in the coupled basis.

$$
\begin{aligned}
d \sigma_{\frac{3}{2}}^{\pi^{0}} & \propto \mid\left\langle\pi ; 1,0\left|\left\langle N ; \frac{1}{2}, \frac{1}{2} \mid \Delta ; \frac{3}{2}, \frac{1}{2}\right\rangle\right|^{2}\right. \\
d \sigma_{\frac{3}{2}}^{\pi^{+}} & \propto \mid\left\langle\pi ; 1,1\left|\left\langle N ; \frac{1}{2},-\frac{1}{2} \mid \Delta ; \frac{3}{2}, \frac{1}{2}\right\rangle\right|^{2}\right.
\end{aligned}
$$

An important constraint then comes from expanding the final physical states into $\pi N$ basis states of definite total isospin using the Clebsh Gordon coefficients.

$$
\begin{aligned}
& |\pi ; 1,0\rangle\left|N ; \frac{1}{2}, \frac{1}{2}\right\rangle=\sqrt{\frac{2}{3}}\left|\pi N ; \frac{3}{2}, \frac{1}{2}\right\rangle-\sqrt{\frac{1}{3}}\left|\pi N ; \frac{1}{2}, \frac{1}{2}\right\rangle \\
& |\pi ; 1,1\rangle\left|N ; \frac{1}{2},-\frac{1}{2}\right\rangle=\sqrt{\frac{1}{3}}\left|\pi N ; \frac{3}{2}, \frac{1}{2}\right\rangle+\sqrt{\frac{2}{3}}\left|\pi N ; \frac{1}{2}, \frac{1}{2}\right\rangle
\end{aligned}
$$

From this constraint it is easy to see that the following relationships can be obtained.

$$
\begin{aligned}
d \sigma_{\frac{3}{2}}^{\pi^{0}} & =\frac{2}{3} d \sigma_{\frac{3}{2}} \\
d \sigma_{\frac{3}{2}}^{\pi^{+}} & =\frac{1}{3} d \sigma_{\frac{3}{2}}
\end{aligned}
$$

It is understood that the cross sections referenced here are evaluated at the $W=$ $1.232 \mathrm{GeV}$ point, that is, near the peak position of the $\Delta$ resonance. The "d" in front of the cross sections will be dropped from now on, it is understood that we are comparing the differential cross sections with no dependent variables specified for 
the distributions. From what was learned the following inequality is obviously true.

$$
\begin{gathered}
\frac{\sigma_{\frac{3}{2}}^{\pi^{+}}}{\sigma_{i n}} \leq \frac{\sigma_{\frac{3}{2}}^{\pi^{0}}}{\sigma_{i n}} \leq \frac{\sigma_{\frac{3}{2}}}{\sigma_{i n}} \\
\tilde{\epsilon} \equiv \frac{\sigma_{\frac{1}{2}}+\sigma_{m}}{\sigma_{\frac{3}{2}}} \ll 1 \\
\epsilon_{1} \equiv \frac{\sigma_{\frac{1}{2}}^{\pi^{0}}+\sigma_{m}^{\pi^{0}}}{\sigma_{\frac{3}{2}}^{\pi^{0}}} \ll 1 \\
\epsilon_{2} \equiv \frac{\sigma_{\frac{1}{2}}^{\pi^{+}}+\sigma_{m}^{\pi^{+}}}{\sigma_{\frac{3}{2}}^{\pi^{+}}} \ll 1
\end{gathered}
$$

Using Eqn. 9.14 and assuming that the isospin $\frac{3}{2}$ contribution is in all cases dominant, one can obtain a useful result.

$$
\begin{aligned}
\sigma^{\pi^{0}} & =\frac{2}{3} \sigma_{\frac{3}{2}}\left(1+\epsilon_{1}\right) \\
\sigma^{\pi^{+}} & =\frac{1}{3} \sigma_{\frac{3}{2}}\left(1+\epsilon_{2}\right) \\
\sigma_{i n} & =\sigma_{\frac{3}{2}}(1+\tilde{\epsilon})
\end{aligned}
$$

This means that the plotted quantity should be approximately

$$
\frac{\sigma^{\pi^{0}}}{\sigma_{\text {in }}} \simeq \frac{2}{3}-\tilde{\epsilon}
$$

So the plotted ratio should be larger than $\frac{1}{3}$ and smaller than $\frac{2}{3}$ near the $\Delta$ resonant position. This constraint is respected by the data in Fig. 9.2. It should be noted that in this section all electromagnetic and weak decay processes were ignored. This is justified due to their small strengths compared to the strong mechanisms.

\subsection{Setting Overlap Check}

The data for the Jefferson Lab E01-002 experiment has two "passes" for the low $Q^{2}$ SOS spectrometer setting, that is, for the setting with $Q^{2}=6.36 \mathrm{GeV}^{2}$ at the $\Delta(1232)$ position. While it is clearly advantageous to add these settings together from a statistical error standpoint, comparing the results obtained by their 
independent analysis is an important systematic check especially in view of the acceptance issues addressed in Section 8.3.2. Figures 9.3 and 9.4 show the HMS spectrometer angles and momenta for each of these passes. The invariant mass (W) contours for planar kinematics are shown superimposed. Each setting is represented by a rectangle to show the typical momentum and angle acceptance. Within each pass one can see that the angular ranges do not overlap much, but that the settings from the other pass will have maximal overlap in angle with the first pass. The

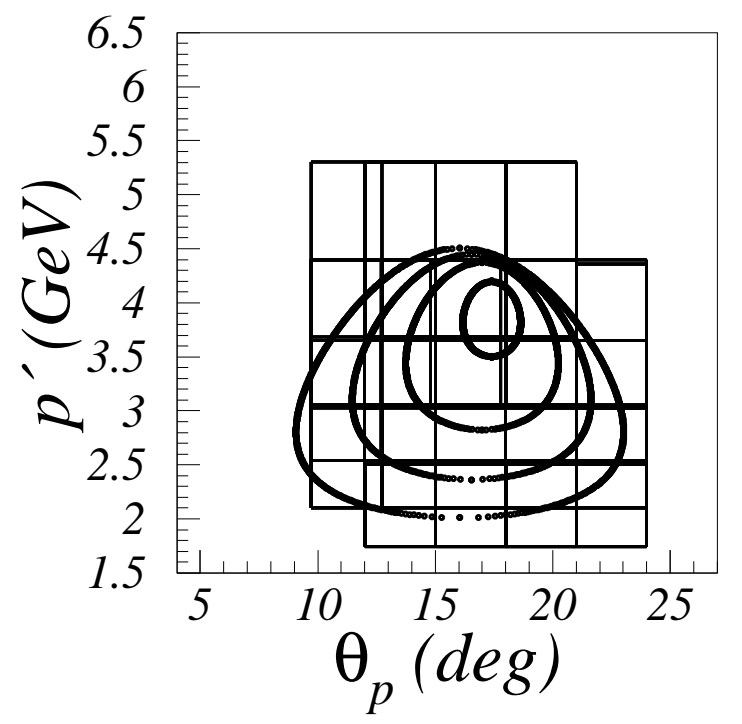

Figure 9.3: Overlap pass 1 for $Q^{2}=6.36$ SOS setting.

contours on the plots represent the planar kinematics for $\mathrm{W}=1.1,1.2,1.3$ and 1.4 $\mathrm{GeV}$ from inside to out. It is easy to see from the superimposed contours that the two groups of HMS settings cover the full kinematic range independently of each other. If one extracts the differential cross section for $\pi^{0}$ production via the prescription discussed in Section 8.7 one obtains the plot displayed in Fig. 9.5. Note that here we deal with the cross section provided by nature, i.e. without radiative corrections. It is this cross section that is more closely related to the possible acceptance issues we are investigating. Indeed, it was explained in Section 4.3 that this is the observable cross section and any other corrected version is to match a theoretical idealization. The agreement of these cross sections seems relatively strong, with few exceptions within statistical errors. It might be useful to have a 


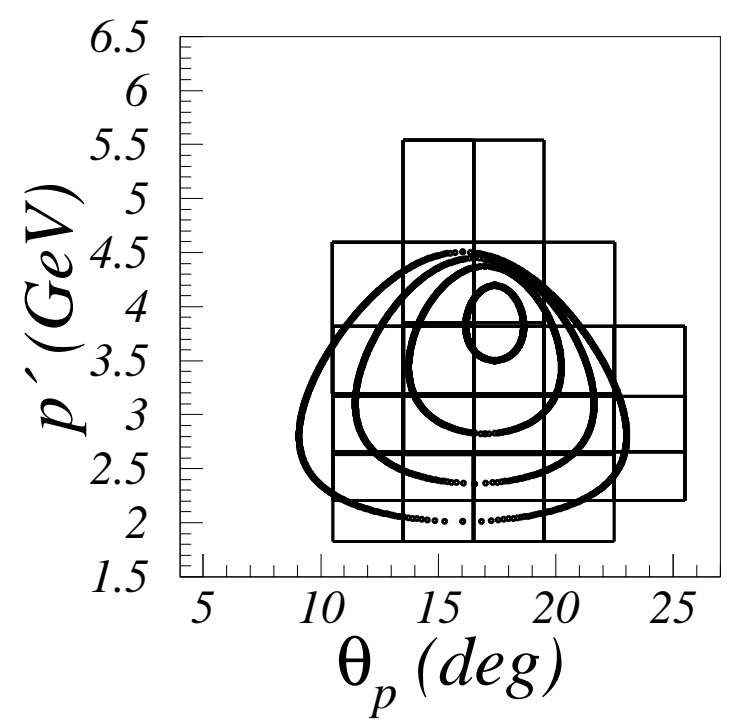

Figure 9.4: Overlap pass 2 for $Q^{2}=6.36$ SOS setting.

look at the $\phi^{*}$ distributions of the same $W=1.232 \mathrm{GeV}$ cross sections. This will allow any possible correlations to be identified. Figure 9.6 shows that there may be some correlations with the differences in the highest $\cos \theta^{*}$ bins at the edges of the $\phi^{*}$ distribution. For example it seems that the pass 2 points may be systematically high. The points have rather large error and the rest of the distribution seems to have strong agreement. It is good practice to use an objective statistical method and not just human judgement. In this particular test there were eight $\mathrm{W}$ bins where the differences between pass 1 and pass 2 could be plotted. One would like to know if the differences of the measured values are consistent with zero for the most part. Because of the relatively small number of trials present (we consider the data set eight trials with 36 points in each) it is difficult to make a strong statement either way but one can easily test for large systematic deviations. One fairly robust statistical method for testing if two data sets came from the same parent distribution is the Kolmogorov-Smirnov test [58]. The Kolmogorov-Smirnov test in its original form is suited to one dimensional distributions. There are attempts at generalizing this statistical test to two dimensions [67] but this will require approximately 160 two dimensional data points and is therefore unsuitable here. Since the robust test which directly tests the statistical similarities of the parent distributions is 


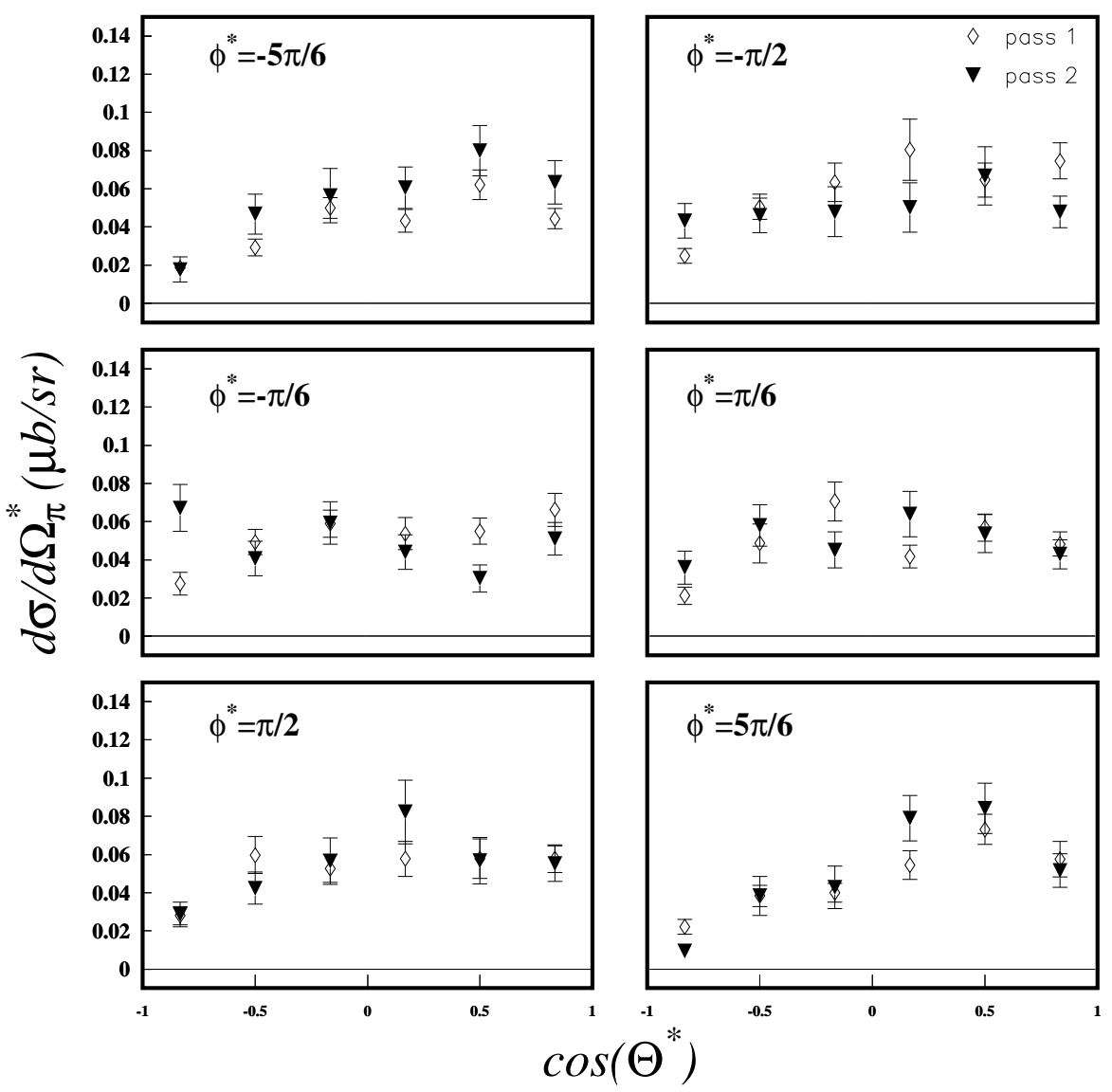

Figure 9.5: Comparison of non radiatively corrected differential cross sections at $W=1.232 \mathrm{GeV}$ and $Q^{2}=6.36 \mathbf{G e V}^{2}$.

unsuitable, we resort to the simpler chi-squared test on the differences, assuming that the theoretical prediction should be zero [51]. Table 9.1 shows the results of a chi-squared analysis on the gathered data. The data in Tbl. 9.1 shows that there may be a systematic shift to larger $\chi^{2}$ since the distribution should be peaked around $n_{\text {dof }}$ with a reasonable probability of being below that mark. Despite this fact it is not terribly unlikely for one to find two data sets whose difference is consistent with zero and has a value of $\chi^{2}$ higher than the total value listed in Tbl. 9.1, this occurrence will happen roughly $25 \%$ of the time. So long as one is sure that all points are not shifted from zero in one direction, there is reasonable statistical agreement between the extractions from each data set. 


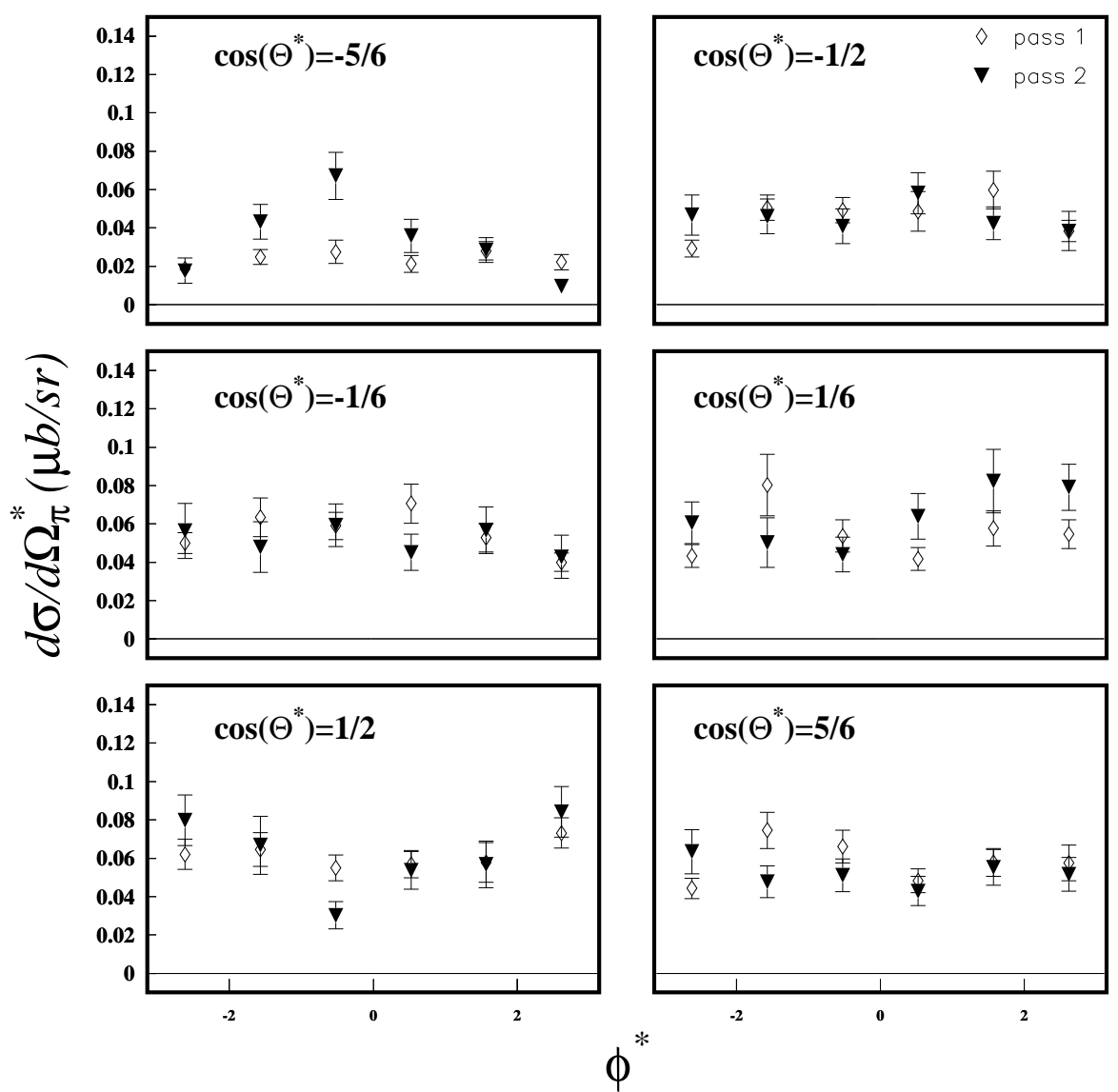

Figure 9.6: Comparison of non radiatively corrected differential cross sections at $W=1.232 \mathrm{GeV}$ and $Q^{2}=6.36 \mathrm{GeV}^{2}$.

\begin{tabular}{|c|c|c|c|}
\hline W range $(\mathrm{GeV})$ & $n_{\text {dof }}$ & $\chi^{2} / n_{\text {dof }}$ & $\int_{\chi^{2}}^{\infty} f(x) d x(\%)$ \\
\hline $1.092 \leq W<1.132$ & 36 & 1.12 & $\sim 32 \%$ \\
\hline $1.132 \leq W<1.172$ & 36 & 1.18 & $\sim 32 \%$ \\
\hline $1.172 \leq W<1.212$ & 36 & 1.38 & $\sim 10 \%$ \\
\hline $1.212 \leq W<1.252$ & 36 & 1.76 & $\sim 1 \%$ \\
\hline $1.252 \leq W<1.292$ & 36 & 1.26 & $\sim 25 \%$ \\
\hline $1.292 \leq W<1.332$ & 36 & 1.14 & $\sim 32 \%$ \\
\hline $1.332 \leq W<1.372$ & 36 & 1.08 & $\sim 50 \%$ \\
\hline $1.372 \leq W<1.402$ & 36 & 1.05 & $\sim 50 \%$ \\
\hline Total & 288 & 1.26 & $\sim 25 \%$ \\
\hline
\end{tabular}

Table 9.1: $\chi^{2}$ values for pass comparison.

\subsection{SOS acceptance issues}

Accurate results in the current experiment depend on modeling the detector acceptance as precisely as possible. It is known, however, that the SOS detector 
acceptance is somewhat more poorly known than the HMS acceptance. There has been an attempt to quantify this uncertainty using both past world data and singlearm inelastic data taken using the better understood HMS spectrometer [16]. In the end the uncertainty of the SOS spectrometer acceptance was estimated to be $5.0 \%$ on average but with stronger local variations (as large as 20.0\%). In the current analysis a simple overall systematic uncertainty of $5.0 \%$ will be included. 


\section{CHAPTER 10 \\ Results and Systematic Errors}

\subsection{The Tie That Binds}

All of the preparation and analysis can now be brought together for the goal of extracting meaningful physics from the data. Of course, the first bit of physics that essentially needs no further interpretation is the differential cross sections for the process of interest. There are other bits of physics obtainable from the functional form of the cross section which will be extracted in Section 10.3. There is one hurdle remaining, which has been mentioned throughout this work. The hurdle is systematic errors. These errors have complicated interdependence and depend strongly on the particular measuring system. There is no clear cut way to deal with this type of error, as opposed to statistical error where one can ask "what happens if I repeat this experiment $\mathrm{N}$ times?" For statistical error the question allows one to use a distribution of possible answers to put an approximate error on the observed value. On the other hand, for systematic errors the answer to the question is easier, yet more troublesome. If you repeat an experiment $\mathrm{N}$ times the systematic error does not change. Therefore one could be systematically off by some factor and never even know it. This situation is unacceptable so some methods must be used to deal with it.

\subsection{Systematic Errors}

We stick to the mantra above and say that a systematic error is one which can never be remedied by repetition of an experiment. This means that if one has a non-unity efficiency in a detector, the error on the correction factor that one must apply is a systematic error. Efficiency corrections and their errors are easy to deal with and one can report this sort of systematic error clearly. Indeed, there have been several efficiency corrections mentioned throughout this work. As long as one is reasonably certain about the efficiency value (presumably from a calibration or previous measurement) one can propagate the error on this value 
to the directly observed physical quantities. Unfortunately efficiencies are not the only way that systematic error can creep into an experiment, there are far more subtle ways. For example, if one uses a physical model to extract an observable the result may depend on the accuracy of the physical model. If the extraction is complicated enough the dependence of the answer on the physical model may be hard to quantify. An example that will be present in this work is the physical model used to correct for the acceptance of the detectors. In situations where it is difficult to quantify the systematic error a priori, a useful tool is a sensitivity study. For the most complicated systematic errors of this experiment this is the method that will be used. Essentially one will vary the parameters which are a likely source of systematic errors within some reasonable bounds. If the final result is not very sensitive to this change, then it is likely that the systematic error due to this parameter is small.

The two different general types of systematic error mentioned above cover most physical situations and are a means of classification for the errors on our extracted observables.

1. Efficiency Systematic Error: Lack of accuracy (not necessarily precision) which results from an inherent inaccuracy of a scale factor to a direct experimental observable.

2. Aggregate Systematic Error: Lack of accuracy which results from effects which are too complicated to quantify by a simple scale factor with transparent meaning. A sensitivity analysis should be done in this case. These errors cannot be remedied by a factor, the analysis is stuck with them unless there is a way to circumvent the cause in the observable extraction.

The quantification of these types of error will give an accurate account of the systematic errors on the measured cross sections because these relate in a simple algebraic way to a direct observable, number of counts. On the other hand quantification of systematic errors on observables extracted by a fitting method can not easily be propagated from the systematic errors on the points used in the fit. In this way, sensitivity studies for the largest suspected errors should be conducted for an ob- 
servable extracted by a fitting method. It is useful at this point to list possible sources of systematic error and how they are dealt with. Table 10.1 displays each important systematic error. The type is noted (efficiency or aggregate). The typical size of the error introduced on the final measurement is noted. The action field indicates which action was taken to combat this error. The symbol "C" stands for a simple correction for a factor and using a nominal systematic error on the factor. An "E" designation means that no correction has been applied for the error but the induced systematic error has been calculated. The symbol "CE" denotes correcting for a factor and computing the error on that factor. The symbol "SS" denotes a sensitivity study, this is common for aggregate type errors. Finally the symbol "CS" stands for corrected in simulation. This means that a parameter was changed in the signal Monte Carlo simulation in order to "correct" for an error in the data. The shift on the simulation parameter is determined by a calibration type study and no additional error is ascribed to the shift. It is assumed that all corrections

\begin{tabular}{|c|c|c|c|c|}
\hline Error & Type & Size & Action & Section \\
\hline beam current & efficiency & $0.5 \%$ & $\mathrm{C}$ & 6.1 \\
\hline proton absorption & efficiency & $1.0 \%$ & $\mathrm{CE}$ & 6.5 \\
\hline fiducial cuts & efficiency & $0.5 \%$ & $\mathrm{E}$ & - \\
\hline collimator cuts & efficiency & $0.5 \%$ & $\mathrm{E}$ & - \\
\hline target boiling & efficiency & $<0.5 \%$ & $\mathrm{E}$ & 6.4 \\
\hline Cerenkov-calorimeter cut & efficiency & $1.6 \%$ & $\mathrm{E}$ & 7.3 .4 \\
\hline HMS D.C. efficiency & efficiency & $0.1 \%$ & $\mathrm{C}$ & 6.3 \\
\hline SOS D.C. efficiency & efficiency & $0.1 \%$ & $\mathrm{C}$ & 6.3 \\
\hline electronic deadtime & efficiency & $0.1 \%$ & $\mathrm{C}$ & 6.6 \\
\hline computer deadtime & efficiency & $0.1 \%$ & $\mathrm{C}$ & 6.6 \\
\hline$M_{x}^{2}$ cut & aggregate & $0.35-2.8 \%$ & $\mathrm{SS}$ & 10.4 .1 \\
\hline radiative cut & aggregate & $0.35-2.8 \%$ & $\mathrm{SS}$ & 10.4 .2 \\
\hline SOS acceptance & efficiency & $5.0 \%$ & $\mathrm{E}$ & 9.4 \\
\hline$\pi^{0}$ radiative & efficiency & $1.0-2.0 \%$ & $\mathrm{E}$ & 8.7 \\
\hline target walls & efficiency & $1.0 \%$ & $\mathrm{E}$ & 7.4 .2 \\
\hline
\end{tabular}

Table 10.1: Systematic errors for E01-002 extracted cross sections. The size reported for is the final estimated error on the experimental result.

which are calculated on a run by run basis, like the deadtimes have a nominal $0.1 \%$ 
uncertainty. This corresponds to approximately 10,000 events used to calculate the deadtimes. An experimental run in E01-002 usually had at least this many events. Before a detailed study of some of the more complicated errors can be pursued,

\begin{tabular}{|c|c|c|c|c|}
\hline Correction & Type & Size & Action & Section \\
\hline beam current & efficiency & $0.5 \%$ & $\mathrm{C}$ & 6.1 \\
\hline proton absorption & efficiency & $4.0 \%$ & $\mathrm{CE}$ & 6.5 \\
\hline HMS D.C. efficiency & efficiency & $5.0 \%$ & $\mathrm{C}$ & 6.3 \\
\hline SOS D.C. efficiency & efficiency & $3.0 \%$ & $\mathrm{C}$ & 6.3 \\
\hline electronic deadtime & efficiency & $0.49 \%$ & $\mathrm{C}$ & 6.6 \\
\hline computer deadtime & efficiency & $6.8 \%$ & $\mathrm{C}$ & 6.6 \\
\hline$M_{x}^{2}$ resolution match & efficiency & $2.0 \%$ & $\mathrm{CE}$ & - \\
\hline target position & aggregate & - & $\mathrm{CS}$ & - \\
\hline multiple scattering & efficiency & - & $\mathrm{CS}$ & 4.5 \\
\hline
\end{tabular}

Table 10.2: Efficiency corrections for E01-002 extracted cross sections. The size reported for is the size (or average size) of the correction factor applied to the data.

one must examine the methods used to extract all important physical information from the experimental data. Some of these methods, particularly those pertaining to extraction of differential cross section, have been discussed in Section 8.7. The next section will reiterate these methods as well as define the methods used to extract more subtle physical observables from the data. Armed with this information Section 10.4 will give a detailed account of selected systematic studies.

\subsection{Physical Extractions}

Section 2.2 was concerned with obtaining the differential cross section for the meson production process in a useable form. It was noted that the electromagnetic part of the process simply factors into the so called "virtual photon flux." With this factorization one can easily isolate the photon-hadron interaction with the understanding that the new "photon" need not be on its mass shell.

$$
\frac{d \sigma}{d E^{\prime} d \Omega_{e} d \Omega_{\pi}^{*}}=\Gamma \frac{d \sigma^{\gamma^{*}}}{d \Omega_{\pi}^{*}}
$$


The procedure outlined in Section 8.7 will do to extract the properly normalized photon-hadron center of mass cross section $\frac{d \sigma^{\gamma^{*}}}{d \Omega_{\pi}^{*}}$. It should be noted that Eqn. 10.1 is written as differential in the electron variables $E^{\prime}$ and $\Omega_{e}$, although the cross sections are reported as functions of $W, Q^{2}$, and $\phi_{e}$. This is legal so long as one remembers that if an integrated cross section is desired the integration should either be carried out in terms of the electron lab variables $E^{\prime}$ and $\Omega_{e}$ or a Jacobian should be included. Focusing on the $W=1.232 \mathrm{GeV}$ data bin will do to understand the extraction procedures and note the approximate level of statistical error. All data points will be reported in Appendix E. Recall that when written explicitly in the center of mass frame the differential photon-hadron cross section can be written as follows [32].

$$
\frac{d \sigma^{\gamma^{*}}}{d \Omega_{\pi}^{*}}=\frac{d \sigma_{T}}{d \cos \theta^{*}}+\epsilon \frac{d \sigma_{L}}{d \cos \theta^{*}}+\epsilon \frac{d \sigma_{T T}}{d \cos \theta^{*}} \cos 2 \phi^{*}+\sqrt{2 \epsilon(1+\epsilon)} \frac{d \sigma_{L T}}{d \cos \theta^{*}} \cos \phi^{*}
$$

The $\phi^{*}$ dependence is already constrained via the spacial rotation properties of the photon density matrix and its symmetry ${ }^{1}$. The $\cos \theta^{*}$ dependence is carried in the "response functions" which have been designated with the labels L,T,LT, or TT. These labels stand for longitudinal, transverse, longitudinal-transverse interference, or transverse-transverse interference respectively. This separation helps for comparison to real photon pion production. Though the $\phi^{*}$ dependence is now obvious the possible $\cos \theta^{*}$ dependence is not so easily constrained unless one restricts himself to states of definite angular momentum or at least states with some finite and small set of definite angular momentum contributions.

Since detailed partial wave expansions are beyond the scope of this work, and also since p-wave dominates to the extend that the $\Delta(1232)$ contribution dominates, the series expansion will only include s and $\mathrm{p}$ waves. Indeed, the $\Delta(1232)$ resonance is sometimes called the $P_{33}$, its spectroscopic symbol, where the "P" represents the orbital angular momentum of the pion-nucleon decay mode. The $\cos \theta^{*}$ dependent cross sections of Eqn. 10.2 can then be written explicitly and fit to experimental data. The dependence that one obtains by including only the lowest two angular

\footnotetext{
${ }^{1}$ The symmetry of the photon density matrix is clearly related to parity invariance as noted in previous sections.
} 
momentum quanta is quoted in several references $[32,41]$. It is then possible to write the $\mathrm{s}$ and $\mathrm{p}$ wave expansion in terms of three unknown functions which depend on $W$ and $Q^{2}$ and are well defined functions of $\cos \theta^{*}$ but not functions of $\phi^{*}$.

$$
\frac{d \sigma^{\gamma^{*}}}{d \Omega_{\pi}^{*}}=A\left(\cos \theta^{*}\right)+\epsilon B\left(\cos \theta^{*}\right) \cos 2 \phi^{*}+\sqrt{2 \epsilon(1+\epsilon)} C\left(\cos \theta^{*}\right) \cos \phi^{*}
$$

The $\sigma_{L}$ and $\sigma_{T}$ contributions get lumped into one representative parameter, $A$, since the experiment at hand does not vary $\epsilon$ and therefore cannot separate these contributions. Using the truncated partial wave expansion one can then write the explicit angular dependence.

$$
\begin{aligned}
& A\left(\cos \theta^{*}\right) \equiv A_{0}+A_{1} \cos \theta^{*}+A_{2} \cos ^{2} \theta^{*} \\
& B\left(\cos \theta^{*}\right) \equiv B_{0} \sin ^{2} \theta^{*} \\
& C\left(\cos \theta^{*}\right) \equiv\left(C_{0}+C_{1} \cos \theta^{*}\right) \sin \theta^{*}
\end{aligned}
$$

The parameters $A_{i}, B_{i}$, and $C_{i}$ are now only functions of the electron variables $W$ and $Q^{2}$, and not functions of the hadronic center of mass angles. A simple way to proceed in characterizing the extracted cross sections is to fit the angular distributions in each $W$ and $Q^{2}$ bin independently. This is the point of view advocated in this work, since to include the $W$ and $Q^{2}$ dependence in the fitting procedures requires detailed knowledge of the dynamics at least at a level where one can add many resonance and background contributions with enough free parameters to obtain a reasonable parameterization. An "energy dependent" analysis, that is, one that includes the dependence on the electron kinematic variables in the fit will be pursued by various groups, when this experimental data is included in the world repository of pion production data. Figure 10.1 shows an example of fit results using the low $Q^{2}$ experimental data and the $W=1.232 \mathrm{GeV}$ center of mass energy bin. The previously described fit is superimposed onto the data. This sample illustrates the procedure for energy independent analysis of the differential cross sections. The binning represented in Fig.10.1 has a $40 \mathrm{MeV}$ wide $\mathrm{W}$ bin centered on $W=1.232 \mathrm{GeV}$ along with six angular bins in $\cos \theta^{*}$ and $\phi^{*}$. This binning is not the final binning for the reporting of the differential cross sections but is used here 

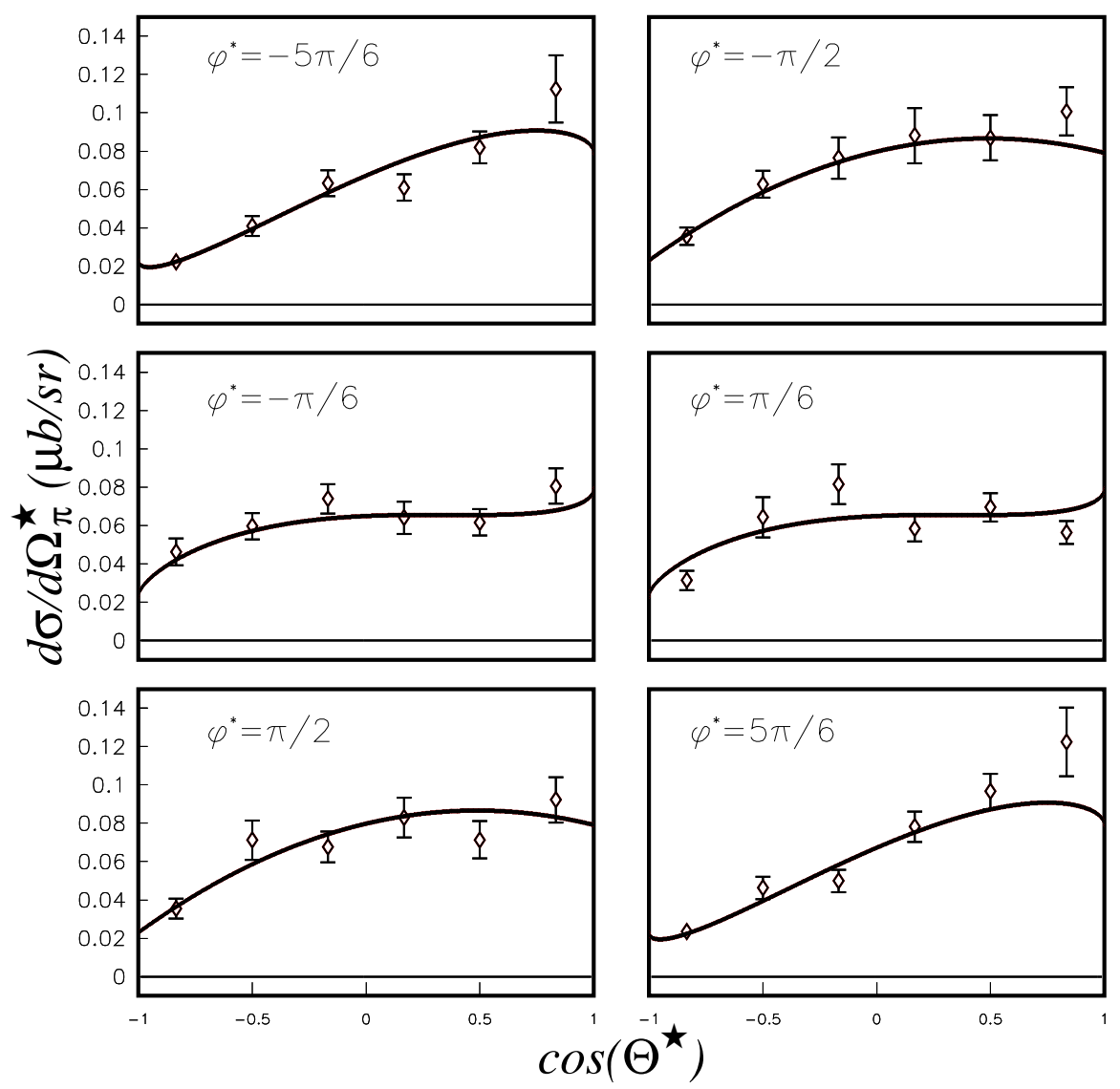

Figure 10.1: Fit to differential cross sections at $W=1.232 \mathrm{GeV}$ and $Q^{2}=$ $6.36 \mathrm{GeV}^{2}$.

for illustrative purposes and to keep statistical errors small. An important feature of the data should be its $\phi^{*}$ symmetry. Since the measurement is unpolarized one should observe only symmetric distributions in $\phi^{*}$ as was discussed in Section 2.2. One can easily see that Fig. 10.2 displays data points which are (to within statistical accuracy) symmetric about the point $\phi^{*}=0$. This fact is a good check on any extracted cross section. The $\phi^{*}$ symmetery is a general feature of the cross sections displayed in Section E.1.

All of the other experimental observables can be extracted from these types of fits by assigning certain physical significance to the fit parameters. The extracted cross sections in the final angular binning scheme are reported in Appendix E for all $\mathrm{W}$ bins. 

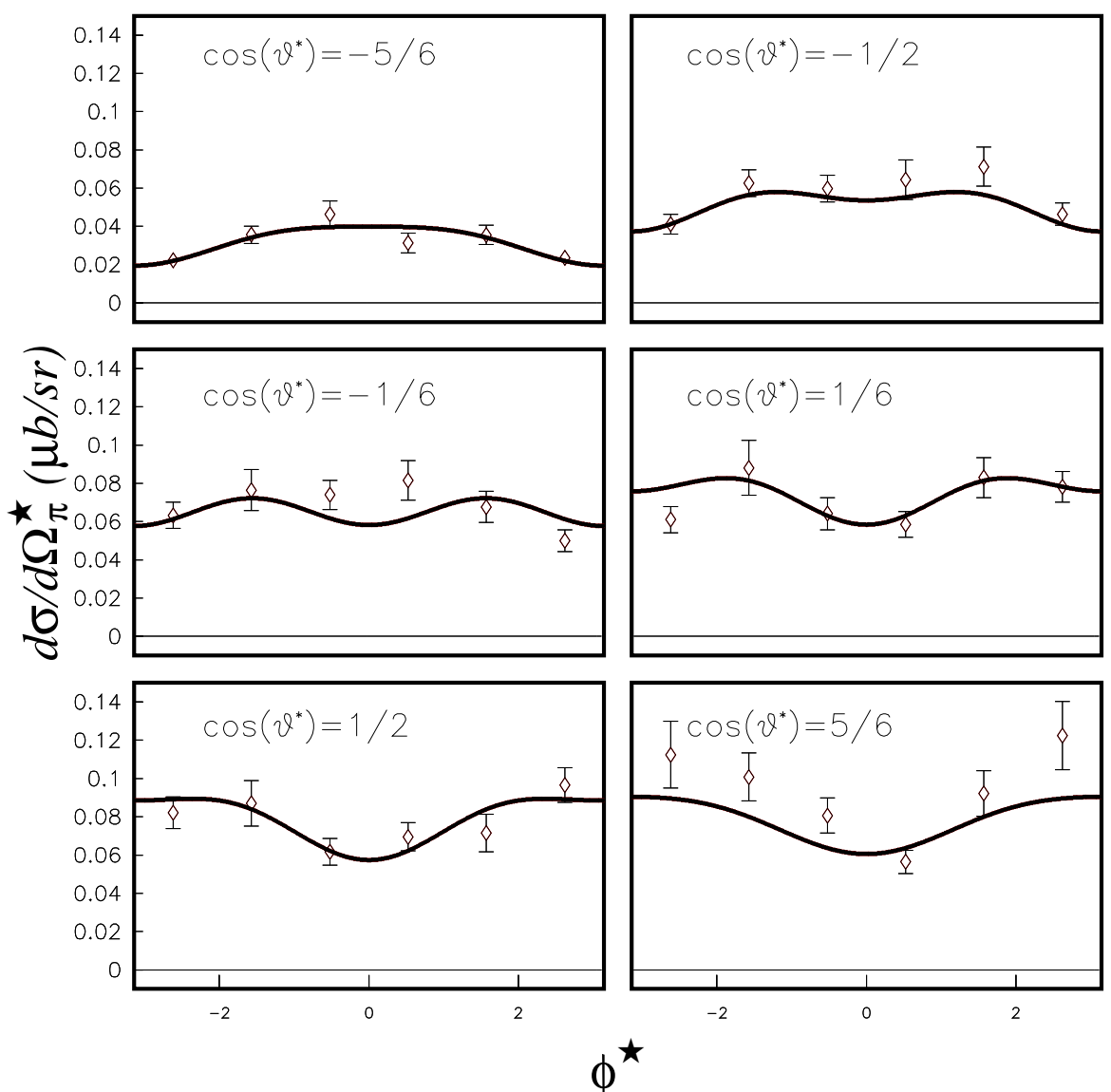

Figure 10.2: Fit to differential cross sections at $W=1.232 \mathrm{GeV}$ and $Q^{2}=$ $6.36 \mathrm{GeV}^{2}$. The data is binned in $\cos \theta^{*}$ and displayed as a function of $\phi^{*}$.

\subsubsection{Multipole Ratios}

The fit parameters used in the last section had but one assumption in their use, namely, that they included only up to P-wave contributions. The $\chi^{2}$ parameters for these fits are fairly good (see Section E.1) and therefore one has confidence for at least the low $Q^{2}$ settings that it is not unreasonable to say the $\mathrm{S}$-wave and $\mathrm{P}$-wave contributions dominate. One can now attempt to go further in the interpretation of these parameters. After all, one would like to constrain the CGLN multipoles. Firstly, if one assumes that the $M_{1+}$ multipole dominates, the previous fit parameters can be related to the multipole ratios in a simple way. In general, the previously 
given fit parameters are related to the CGLN multipoles as follows $[62,41]$.

$$
\begin{aligned}
& A_{0}=\frac{2 W\left|\mathbf{k}_{\pi}^{*}\right|}{W^{2}-m_{p}^{2}}\left[\left|E_{0+}\right|^{2}+\left|M_{1-}\right|^{2}+\frac{5}{2}\left|M_{1+}\right|^{2}+\frac{9}{2}\left|E_{1+}\right|^{2}-3 \Re\left(E_{1+}^{*} M_{1+}\right)\right. \\
& +\Re\left(\left(3 E_{1+}+M_{1+}\right) M_{1-}^{*}\right) \\
& \left.+\frac{\epsilon Q^{2}}{\left|\mathbf{q}^{*}\right|^{2}}\left(\left|S_{0+}\right|^{2}+\left|S_{1-}\right|^{2}+4\left|S_{1+}\right|^{2}-4 \Re\left(S_{1+} S_{1-}^{*}\right)\right)\right] \\
& A_{1}=\frac{2 W\left|\mathbf{k}_{\pi}^{*}\right|}{W^{2}-m_{p}^{2}}\left[2 \Re\left(E_{0+}\left(3 E_{1+}+M_{1+}-M_{1-}\right)^{*}\right)+\frac{\epsilon Q^{2}}{\left|\mathbf{q}^{*}\right|^{2}} 2 \Re\left(S_{0+}\left(4 S_{1+}+S_{1-}\right)^{*}\right)\right] \\
& A_{2}=\frac{2 W\left|\mathbf{k}_{\pi}^{*}\right|}{W^{2}-m_{p}^{2}}\left[-\frac{3}{2}\left|M_{1+}\right|^{2}+\frac{9}{2}\left|E_{1+}\right|^{2}+9 \Re\left(E_{1+}^{*} M_{1+}\right)-3 \Re\left(\left(3 E_{1+}+M_{1+}\right) M_{1-}^{*}\right)\right. \\
& \left.12 \frac{\epsilon Q^{2}}{\left|\mathbf{q}^{*}\right|^{2}}\left(\left|S_{1+}\right|^{2}+\Re\left(S_{1+} S_{1-}^{*}\right)\right)\right] \\
& B_{0}=\frac{2 W\left|\mathbf{k}_{\pi}^{*}\right|}{W^{2}-m_{p}^{2}}\left[-\frac{3}{2}\left|M_{1+}\right|^{2}+\frac{9}{2}\left|E_{1+}\right|^{2}-3 \Re\left(E_{1+}^{*} M_{1+}\right)+3 \Re\left(\left(E_{1+}-M_{1+}\right) M_{1-}^{*}\right)\right] \\
& C_{0}=-\frac{2 W\left|\mathbf{k}_{\pi}^{*}\right|}{W^{2}-m_{p}^{2}} \sqrt{\frac{2 Q^{2}}{\left|\mathbf{q}^{*}\right|^{2}}} \Re\left(S_{0+}\left(3 E_{1+}-M_{1+}+M_{1-}\right)^{*}-E_{0+}\left(2 S_{1+}-S_{1-}\right)^{*}\right) \\
& C_{1}=-\frac{2 W\left|\mathbf{k}_{\pi}^{*}\right|}{W^{2}-m_{p}^{2}} 6 \sqrt{\frac{2 Q^{2}}{\left|\mathbf{q}^{*}\right|^{2}}} \Re\left(S_{1+}\left(E_{1+}-M_{1+}+M_{1-}\right)^{*}+S_{1-} E_{1+}^{*}\right)
\end{aligned}
$$

Now, assuming that the $M_{1+}$ multipole dominates all situations, one can eliminate all combinations in the previous equations which do not contain this multipole.

$$
\begin{aligned}
& A_{0}=\frac{2 W\left|\mathbf{k}_{\pi}^{*}\right|}{W^{2}-m_{p}^{2}}\left|M_{1+}\right|^{2}\left[\frac{5}{2}-3 \frac{\Re\left(E_{1+}^{*} M_{1+}\right)}{\left|M_{1+}\right|^{2}}+\frac{\Re\left(M_{1+}^{*} M_{1-}\right)}{\left|M_{1+}\right|^{2}}\right] \\
& A_{1}=\frac{2 W\left|\mathbf{k}_{\pi}^{*}\right|}{W^{2}-m_{p}^{2}}\left|M_{1+}\right|^{2} 2 \frac{\Re\left(E_{0+}^{*} M_{1+}\right)}{\left|M_{1+}\right|^{2}} \\
& A_{2}=\frac{2 W\left|\mathbf{k}_{\pi}^{*}\right|}{W^{2}-m_{p}^{2}}\left|M_{1+}\right|^{2}\left[-\frac{3}{2}+9 \frac{\Re\left(E_{1+}^{*} M_{1+}\right)}{\left|M_{1+}\right|^{2}}-3 \frac{\Re\left(M_{1-}^{*} M_{1+}\right)}{\left|M_{1+}\right|^{2}}\right] \\
& B_{0}=\frac{2 W\left|\mathbf{k}_{\pi}^{*}\right|}{W^{2}-m_{p}^{2}}\left|M_{1+}\right|^{2}\left[-\frac{3}{2}-3 \frac{\Re\left(E_{1+}^{*} M_{1+}\right)}{\left|M_{1+}\right|^{2}}-3 \frac{\Re\left(M_{1-}^{*} M_{1+}\right)}{\left|M_{1+}\right|^{2}}\right] \\
& C_{0}=\frac{2 W\left|\mathbf{k}_{\pi}^{*}\right|}{W^{2}-m_{p}^{2}}\left|M_{1+}\right|^{2} \sqrt{\frac{2 Q^{2}}{\left|\mathbf{q}^{*}\right|^{2}}} \frac{\Re\left(S_{0+}^{*} M_{1+}\right)}{\left|M_{1+}\right|^{2}} \\
& C_{1}=\frac{2 W\left|\mathbf{k}_{\pi}^{*}\right|}{W^{2}-m_{p}^{2}}\left|M_{1+}\right|^{2} 6 \sqrt{\frac{2 Q^{2}}{\left|\mathbf{q}^{*}\right|^{2}}} \frac{\Re\left(S_{1+}^{*} M_{1+}\right)}{\left|M_{1+}\right|^{2}}
\end{aligned}
$$


Using the simple fitting method developed in the introduction to this section and the data displayed in Section E.1 one can obtain values for the angle integrated cross sections and for the multipole interference terms displayed in Eqn. 10.6. The results of the $W$ independent fits for the current experimental data sets are shown in Figs. 10.3 and 10.4. In this way, the fits can give a constraint on the relevant
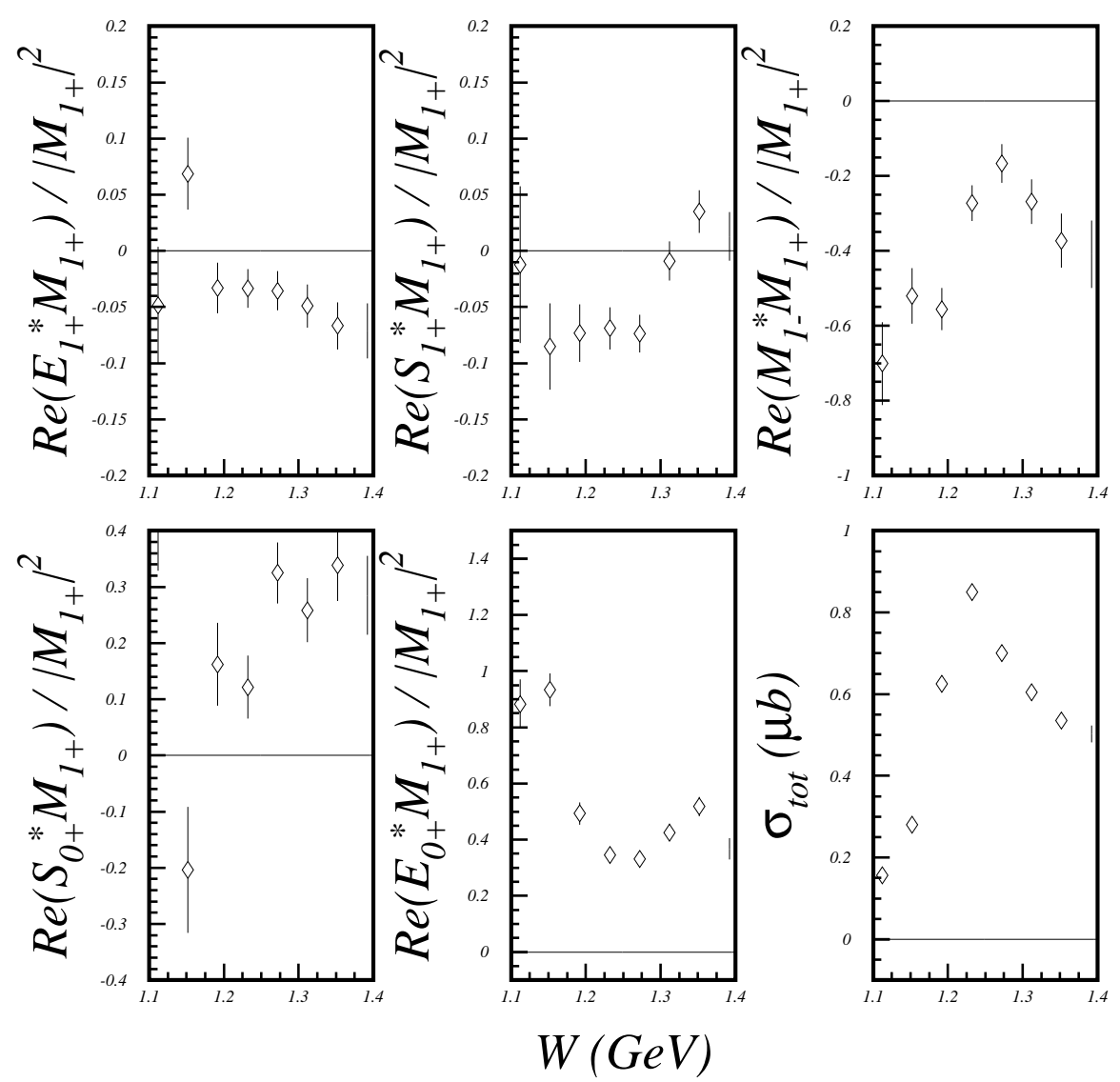

\section{Figure 10.3: Results of the simple $M_{1+}$ dominance fit for the lower $Q^{2}$ data set as a function of invariant energy $W$.}

multipoles. If one further assumes that the resonance is dominant at the pole position (an assumption that becomes less valid with increasing $Q^{2}$ ) the values of $R_{E M}$ and $R_{S M}$ can be extracted from the above fits. The fits for the higher $Q^{2}$ data are largely unconstrained as far as the multipole amplitudes go. The reason for this is that this data has less statistical significance and does not have the ability to constrain slight angular dependencies accurately. The values obtained for the lower $Q^{2}$ data are $R_{E M}=-3.3 \pm 1.7 \%$ and $R_{S M}=-6.9 \pm 1.8 \%$. It is unlikely, 

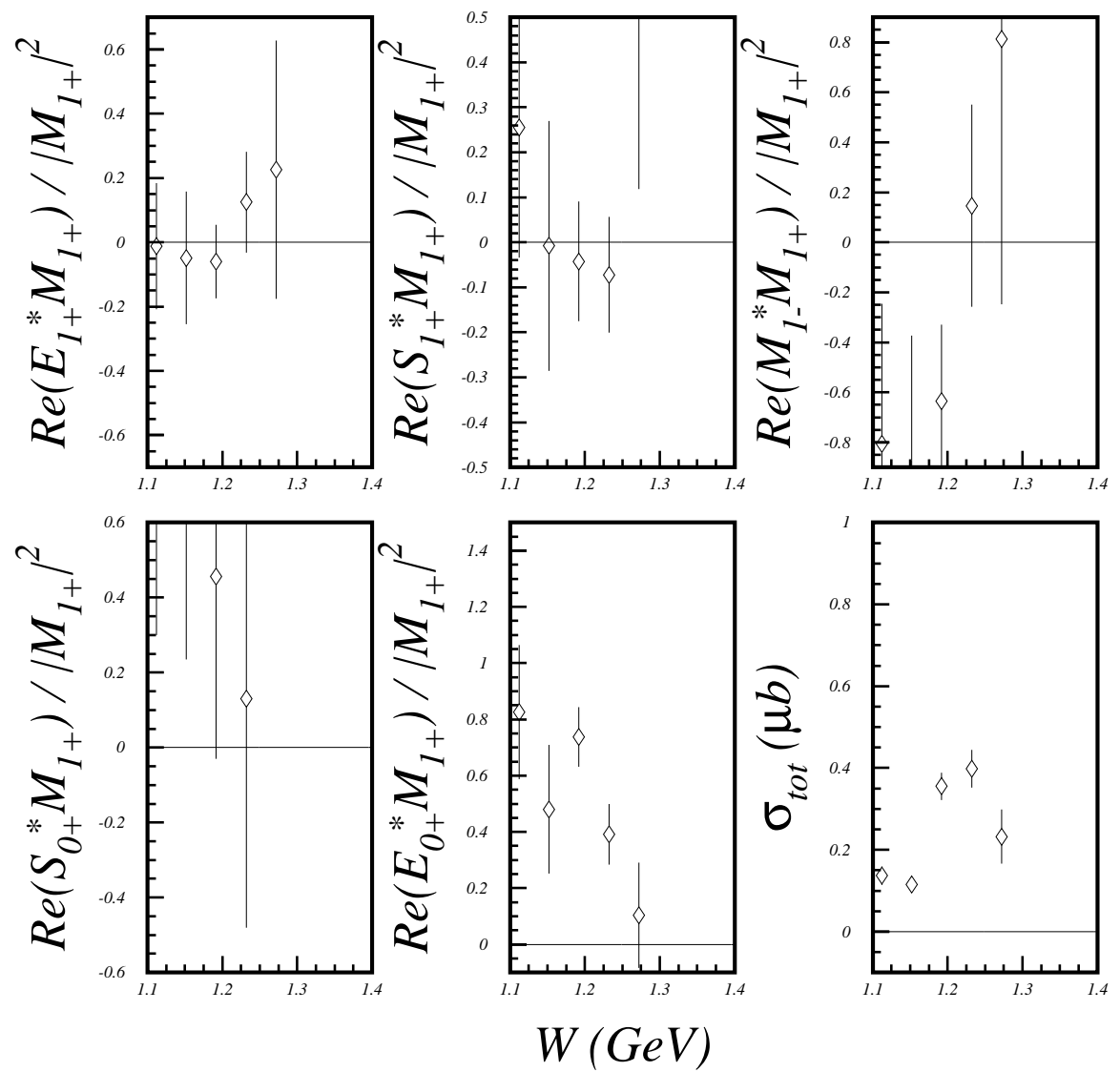

\section{Figure 10.4: Results of the simple $M_{1+}$ dominance fit for the higher $Q^{2}$ data set as a function of invariant energy $W$.}

however that the assumptions used in this fit are true to life. There is a hint that the $E_{0+}$ amplitude is becoming significant compared to the $M_{1+}$. This value of $R_{E M}$ would also represent a value somewhat consistent with previous values which were negative and small in magnitude (few percent). If the ratio was constrained to be above previous values and consistent with zero, this is a hint that $R_{E M}$ is moving toward positive values, and would be very significant.

\subsubsection{Transition Form Factors}

In addition to the multipole ratios the transition form factors (in the Jones and Scadron convention) can be extracted. For the $M_{1+}$ dominant fit, the magnetic form factor $G_{M}^{*}$ will be extract able and directly related to the multipole $M_{1+}$ if the resonance is completely dominant at the peak position. First notice that when one 
integrates the angular distribution quoted in Eqn. 10.3 one obtains $\frac{4 \pi}{3}\left(A_{2}+3 A_{0}\right)$. This expression can easily be put in terms of the multipole amplitudes (assuming, still, $M_{1+}$ dominance) to get:

$$
\sigma=8 \pi \frac{2 W\left|\mathbf{k}_{\pi}^{*}\right|}{W^{2}-m_{p}^{2}}\left|M_{1+}\right|^{2}
$$

One can therefore extract the (presumed dominant) $M_{1+}$ multipole from just a measure of the angle integrated cross section. Further, one has the relation:

$$
\Im\left(M_{1+}^{(3 / 2)}\right)=\eta_{b} \sqrt{\frac{2}{3}} G_{M}^{*}
$$

left over from Eqn. 1.36. Therefore a measurement of the cross section, armed with the assumption that $M_{1+} \simeq i \Im\left(M_{1+}^{(3 / 2)}\right)$, is a direct measurement of the magnetic transition form factor assuming resonance and magnetic multipole dominance.

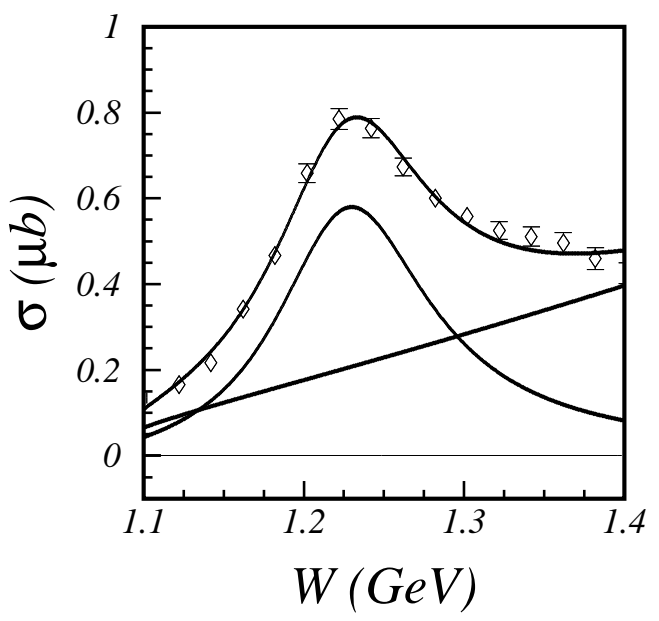

Figure 10.5: The total angle integrated cross section for lower $Q^{2}$ settings fit with a Breit-Wigner function and a fractional power polynomial of second order.

Figures 10.6 and 10.5 display the experimentally observed angle integrated cross sections and fits to them. One can see that the resonance contribution is not necessarily dominant since the background contribution is roughly $50 \%$ and $99 \%$ of the peak height for the lower $Q^{2}$ and higher $Q^{2}$ data respectively. These factors can be used as a rough correction factor to $\left(G_{M}^{*}\right)^{2}$. Figure 10.7 shows the current 


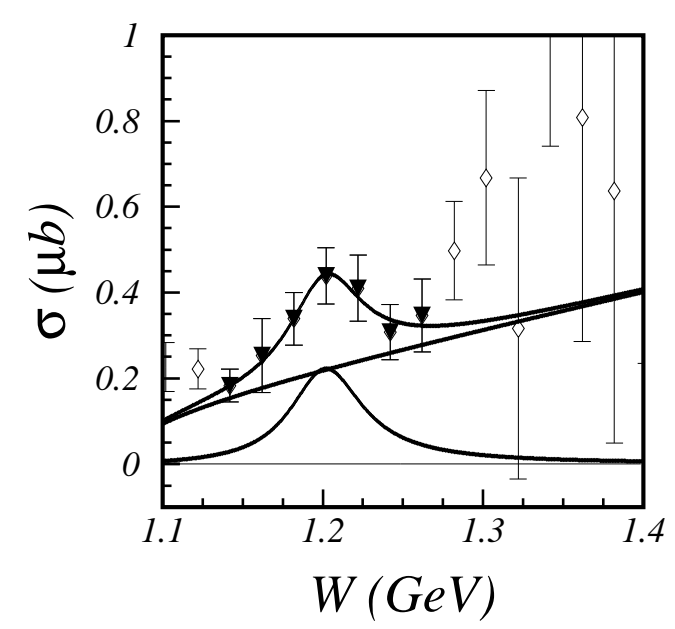

Figure 10.6: The total angle integrated cross section for higher $Q^{2}$ settings fit with a Breit-Wigner function and a polynomial of second order. The dark inverted triangle data points are the only ones used to constrain the fit due to lack of statistics for higher $W$ and the possibility of small elastic radiative contamination at lower $W$.

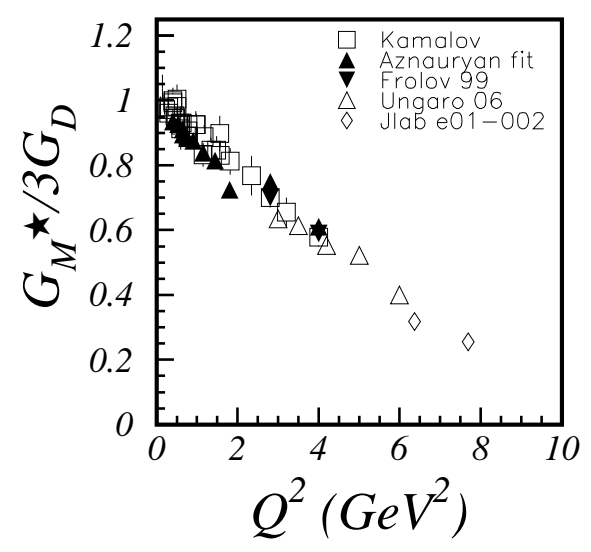

Figure 10.7: Extracted value of the Jones and Scadron $G_{M}^{*}$ transition form factor. The values include this and several other exclusive analyses.

experimental situation for the transition form factor including the values extracted in this work. Note that these values are quite well constrained by the data even at the higher $Q^{2}$ point. In short, our higher $Q^{2}$ data has the ability to give overall cross section sizes but lacks the ability to give detailed information about angular distributions, due to poor statistics. The calculated values of $G_{M}^{*}$ still seem to be 
dropping about $30.0 \%$ faster than the dipole contribution mentioned in Section 1.6. This fact signals that soft processes are probably still important to the $\Delta$ excitation.

\subsection{Systematic Studies}

There are two data restrictions which require systematic studies to quantify the induced error that they cause on the extracted observables. These restrictions are the missing mass restriction and the elastic radiative restriction. The current section outlines a sensitivity study for these restrictions. It is possible for the modifications of these cuts to have some interdependence, this is ignored in the present analysis so that when one studies the elastic radiative restriction all other restrictions remain at the nominal values.

Some systematic errors are easily quantifiable by simple studies. Mostly these are normalization errors like uncertainty in the beam current. If one can accurately compute the values of these uncertainties by some studies then they can be added (in quadrature) as systematic errors. The missing mass and elastic radiative restrictions are not of this type because they depend on somewhat subjective choices of parameters. To quantify these errors one essentially must map out the dependence of the final answer on any arbitrarily defined parameters. To frame this procedure, think of an experimental observable " $f$ " which may depend on an arbitrary analysis parameter " $a$ " as $f(a)$. Now, if one had some concrete way of defining the parameter " $a$ " which is not subjective the problem is solved since a measurement of the uncertainty in " $a$ " can be made and propagated through to the experimental observable. However, here we assume that one does not have a concrete way of defining " $a$ " and that it depends a great deal on the observation of some data distribution. The only option then is to map out the result for different values of " $a$ " and make and report the resulting sensitivity as a systematic error on the measured quantity.

\subsubsection{Missing Mass Restriction}

The missing mass restrictions are known to have some resolution mismatch from the discussion of Section 7.3.2. Even though one can arrive at an approximate correction factor, it is useful to know the error on this factor and the error induced 
by the somewhat arbitrary location of the missing mass data restriction. To do this, as was noted, a sensitivity study is the choice given that a rigorous derivation of this error is lacking. Figure 10.8 shows the various restrictions used to estimate this error. The variation of the cross sections and other extracted observables are
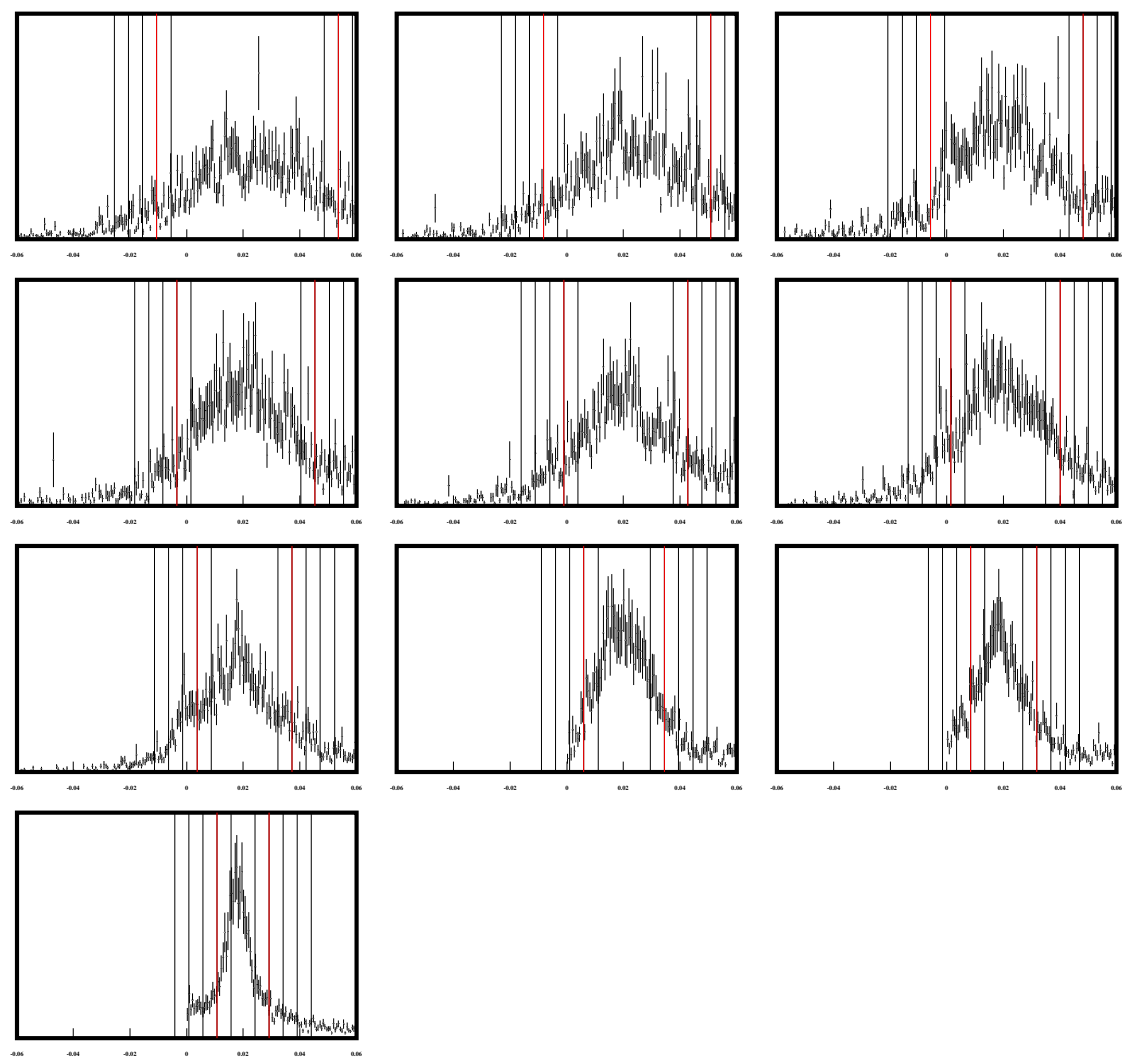

Figure 10.8: Various missing mass restrictions used to probe the systematic error of the nominal width. The highlighted lines represent the nominal width.

monitored with every only the missing mass restrictions displaced from the nominal values. If a simple straight line is fit through the values one can get a determination of the local first order rate of change of the quantities of interest. The scale of the width increments is used as a multiplier for the approximate error. The basic situation is as it would be for a Taylor expansion for a generic $f(a)$.

$$
f(a) \simeq f\left(a_{0}\right)+\left.\frac{d f}{d a}\right|_{a=a_{0}} \delta_{a}
$$


The symbol $a_{0}$ represents the nominal width and $\delta_{a}$ the approximate scale on which $f$ changes, taken to be the width increments. The correction term in Eqn. 10.8 is then taken to be the systematic error on the measured cross section due to the missing mass restriction. Typical values of this error range from $0.35 \%$ to $2.8 \%$. One may also be interested in the systematic error on an observable extracted by a fitting method. Figure 10.9 shows how our estimator for $R_{E M}$ varies due to the missing mass restriction. An estimate for the uncertainty on $R_{E M}$ due to the missing mass

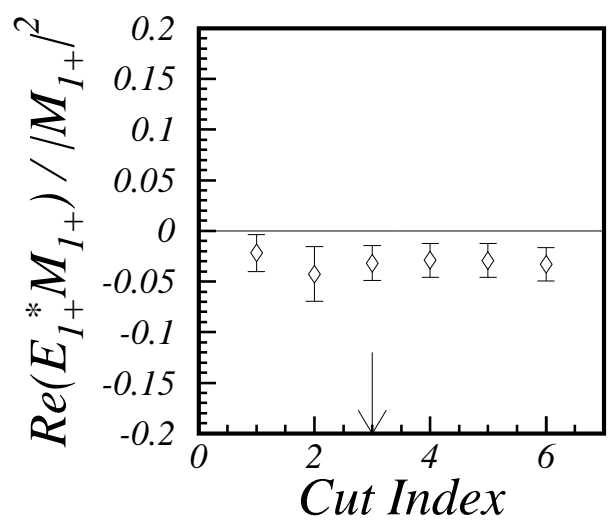

Figure 10.9: The variation of the $R_{E M}$ estimator on missing mass restriction. From left to right the restrictions are labeled from narrowest to widest with the arrow marking the nominal.

restriction is $1.0 \%$, based on this analysis.

\subsubsection{Elastic Radiative Rejection}

An exactly analogous study can be carried out for the elastic radiative rejection. Recall from Section 7.5 that the radiative rejection is broken up into three regions of $\cos \theta^{*}$ with the region corresponding to the most forward going pions left unaltered. The other two regions have a two dimensional restriction. Varying these parameters within reasonable boundaries one can come up with the curves displayed in Figs. 10.10 and 10.11. When one evaluates the error that the uncertainty in these restrictions produce the value is very similar to the uncertainty induced by the missing mass restrictions (or at least in the same range of values for all the points). The error takes values in the range $0.35-2.8 \%$. 


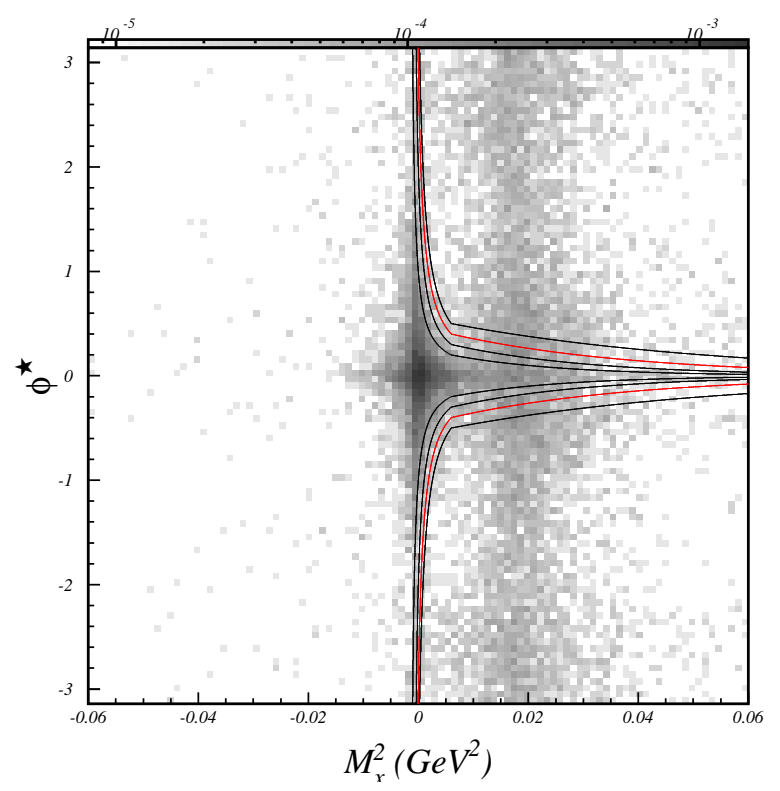

Figure 10.10: Radiative rejection overlay for lowest $\cos \theta^{*}$ bin. Several variations on the nominal rejection curves are displayed. The nominal curves appear as highlighted.

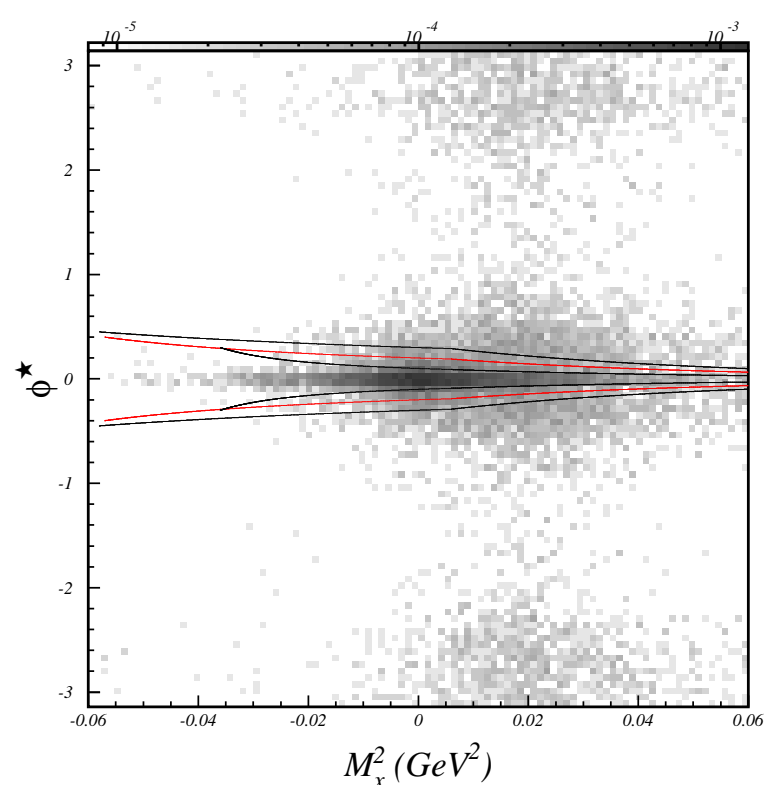

Figure 10.11: Radiative rejection overlay for intermediate $\cos \theta^{*}$ bin. Several variations on the nominal rejection curves are displayed. The nominal curves appear as highlighted. 


\subsection{Conclusions}

This work has accomplished several goals. The first and foremost goal was to extract the center of mass neutral pion electroproduction cross section in the invariant mass region roughly corresponding to the well-known $\Delta$ resonance. This goal was accomplished and the systematic errors on said cross section were evaluated using the best current knowledge of the detector systems and analysis procedures. The next goal of the analysis was to investigate (in a simplified way) what the cross sections suggest for the most important multipole amplitudes and transition form factors relating to the measured process and in particular to the $\Delta$ resonance.

In the realm of a fit which includes only $\mathrm{S}$-wave and $\mathrm{P}$-wave contributions and assumes that the multipole $M_{1+}$ dominates all other multipoles (an assumption which seems to be challenged by the size of $\left.E_{0+}\right)$, with the $\Delta$ resonance being dominant at the resonance position, one can obtain values for $R_{E M}, R_{S M}$ and $G_{M}^{*}$. The specifications of $R_{E M}$ and $R_{S M}$ depend in detail on the angular distribution of the cross section and thus are only well determined for the $Q^{2}=6.36 \mathrm{GeV}^{2}$ data set. The most significant facts that are obtained using these methods are that $R_{E M}=-3.3 \pm 1.7 \%$ and that $G_{M}^{*}$ seems to be still dropping much faster than the simple dipole form. As was reviewed in Chapter 3 , the fact that $G_{M}^{*}$ continues to drop faster than the dipole form suggests that there are soft mechanisms in the $\Delta$ excitation which are still important and the small negative value of $R_{E M}$ (if it stands up to more detailed fitting procedures) indicates that this hadronic observable does not seem to be beginning to interpolate to its pQCD limit [20].

It is important to reiterate the physical effects which were not considered in this work. Everything assumes that single photon exchange is completely appropriate for dealing with observables measured to the accuracy that was displayed in this data. This is probably a good assumption but there are some places, including calculation of radiative effects which two photon exchange mechanisms might have a more important role. These are left to subsequent study. In extracting our estimates for the multipole ratios and transition form factor of the $\Delta$ excitations it was assumed that the $\Delta$ dominates at the resonance position, though this assumption has already been shown to be suspect in Section 10.3.2. Contributions from multipoles other 
than $M_{1+}$, specifically $E_{0+}$ may also be showing their importance and thus spoiling the dominance that was assumed.

A more accurate fitting method which constrains the background and resonant contributions, as well as including other known resonances will be subsequently pursued using this data set, in addition to all the worlds data on the subject to date. This fit should give some insight into some very interesting physics and help to uncover the inner workings of hadronic physics and QCD. 


\section{BIBLIOGRAPHY}

[1] D. Abbott, V. Gyurjyan, G. Heyes, E. Jastrzembski, C. Timmer, and E. Wolin. Jefferson Lab Data Acquisition Run Control System. In CHEP04 Proceedings, 2004.

[2] A. Afanasev, I. Akushevich, V. Burkert, and K. Joo. QED Radiative Corrections in Processes of Exclusive Pion Electroproduction. Phys. Rev., D66:074004, 2002.

[3] J. Ahrens et al. Helicity Dependence of the $\gamma p \rightarrow N \pi$ Channels and Multipole Analysis in the $\Delta$ Region. Eur. Phys. J., A21:323-333, 2004.

[4] H. L. Anderson, E. Fermi, E. A. Long, and D. E. Nagle. Total Cross-Sections of Positive Pions in Hydrogen. Phys. Rev., 85:936, 1952.

[5] C. S. Armstrong et al. Electroproduction of the $S_{11}(1535)$ Resonance at High Momentum Transfer. Phys. Rev., D60:052004, 1999.

[6] Christopher S. Armstrong. Electroproduction of the $S_{11}(1535)$ at High Momentum Transfer. PhD thesis, The College of William and Mary, 1998.

[7] J. Arrington. A-B-SIMC. 18 pages, unpublished, 2001.

[8] John R. Arrington. Inelastic Electron Scattering from Nuclei at $x>1$ and High $Q^{2}$. PhD thesis, California Institute of Technology, 1998.

[9] I. G. Aznauryan et al. Electroexcitation of the $P_{33}(1232), P_{11}(1440), D_{13}(1520)$, $S_{11}(1535)$ at $Q^{2}=0.4-(\mathrm{GeV} / \mathrm{c})^{2}$ and 0.65- $(\mathrm{GeV} / \mathrm{c})^{2}$. Phys. Rev., C71:015201, 2005.

[10] Bettina Bantes. Absolutbestimmung der dominanten Amplituden der Elektroproduktion von neutralen Pionen beim Impulsubertrag von 0.630-GeV ${ }^{2}$. PhD thesis, University of Bonn, 2003. BONN-IR-2003-08. 
[11] D. Yu. Bardin and N. M. Shumeiko. An Exact Calculation of the Lowest Order Electromagnetic Correction to the Elastic Scattering. Nucl. Phys., B127:242, 1977.

[12] C. Becchi and G. Morpurgo. Vanishing of the E2 Part of the $N_{33}^{*} \rightarrow N+\gamma$ Amplitude in the Non-Relativistic Quark Model of "Elementary" Particles. Phys. Lett., 17:352-354, 1965.

[13] P. Benz et al. Measurement of the Reaction $\gamma d \rightarrow \pi^{-} p p$, and Determination of Cross-Sections for the Reaction $\gamma n \rightarrow \pi^{-} p$, at Photon Energies Between 0.2-GeV and 2.0- GeV. Nucl. Phys., B65:158-209, 1973.

[14] Frits A. Berends and A. Donnachie. A Multipole Analysis of Pion Photoproduction in the First Resonance Region. Nucl. Phys., B84:342, 1975.

[15] G. Blanpied et al. $N \rightarrow \Delta$ Transition from Simultaneous Measurements of $p(\vec{\gamma}, \pi)$ and $p(\vec{\gamma}, \gamma)$. Phys. Rev. Lett., 79:4337, 1997.

[16] P. Bosted. Check of SOS Acceptance. private communication, 2007.

[17] V. D. Burkert and T. S. H. Lee. Electromagnetic Meson Production in the Nucleon Resonance Region. Int. J. Mod. Phys., E13:1035-1112, 2004.

[18] Jr. Callan, Curtis G. and David J. Gross. High-Energy Electroproduction and the Constitution of the Electric Current. Phys. Rev. Lett., 22:156-159, 1969.

[19] Simon Capstick and Nathan Isgur. Baryons in a Relativized Quark Model with Chromodynamics. Phys. Rev., D34:2809, 1986.

[20] Carl E. Carlson and Nimai C. Mukhopadhyay. Approach to Perturbative Re-

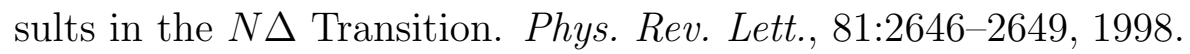

[21] G. F. Chew, M. L. Goldberger, F. E. Low, and Yoichiro Nambu. Relativistic Dispersion Relation Approach to Photomeson Production. Phys. Rev., 106:1345-1355, 1957. 
[22] A. Christ, G. Noldeke, W. Nuding, T. Reichelt, and H. Stanek. Experimental Test for the $1 \geq \Delta I$ (Isospin) Rule in Electromagnetic Transitions. Nucl. Phys., B102:429-438, 1976.

[23] R. W. Clifft et al. A Search for Delta $I=2$ Electromagnetic Currents in Pion Photoproduction. Phys. Rev. Lett., 33:1500, 1974.

[24] T. DeForest Jr. and J. D. Walecka. Electron Scattering and Nuclear Structure. Adv. Phys., 15:1-109, 1966.

[25] J. R. Delayen. Cumulative Beam Breakup in Linear Accelerators with Random Displacement of Cavities and Focusing Elements. Phys. Rev. Special Topics, 7:074402, 2004.

[26] E. Di Capua et al. Results of an Experiment to Test for an $\mathrm{I}=2$ Term in the Electromagnetic Current. Lett. Nuovo Cim., 8S2:692-696, 1973.

[27] Paul A. M. Dirac. Relativistic Wave Equations. Proc. Roy. Soc. Lond., 155A:447-459, 1936.

[28] Norman Dombey. Scattering of Polarized Leptons at High Energy. Rev. Mod. Phys., 41:236-246, 1969.

[29] A. Donnachie, G. Shaw, and D. H. Lyth. Form Factors and Electroproduction. In A. Donnachie and G. Shaw, editors, Electromagnetic Interactions of Hadrons, volume 1, pages 221-256. Plenum Press, New York, 1978.

[30] D. Drechsel and M. M. Giannini. A Note on the Quadrupole $(N \rightarrow \Delta)$ Transition Amplitude in Quark Models. Phys. Lett., B143:329-333, 1984.

[31] D. Drechsel, O. Hanstein, S. S. Kamalov, and L. Tiator. A Unitary Isobar Model for Pion Photo- and Electroproduction on the Proton up to 1-GeV. Nucl. Phys., A645:145-174, 1999.

[32] D. Drechsel and L. Tiator. Threshold Pion Photoproduction on Nucleons. J. Phys., G18:449-497, 1992. 
[33] Dipangkar Dutta and Pat Welch. The Hall C Matrix Element Optimization Package (CMOP). 8 pages, unpublished, 1996.

[34] A. R. Edmonds. Angular Momentum in Quantum Mechanics. Princeton University Press, Princeton NJ, second edition, 1974.

[35] D. Elsner et al. Measurement of the LT-Asymmetry in $\pi^{0}$ Electroproduction at the Energy of the $\Delta(1232)$ Resonance. Eur. Phys. J., A27:91-97, 2006.

[36] R. Ent et al. Radiative Corrections for $\left(e, e^{\prime} p\right)$ Reactions at GeV Energies. Phys. Rev., C64:054610, 2001.

[37] Richard P. Feynman. Photon-Hadron Interactions. Westview APB, USA, 1972.

[38] M. Fierz and W. Pauli. On Relativistic Wave Equations for Particles of Arbitrary Spin in an Electromagnetic Field. Proc. Roy. Soc. Lond., A173:211-232, 1939.

[39] Jerome I. Friedman and Henry W. Kendall. Deep Inelastic Electron Scattering. Ann. Rev. Nucl. Part. Sci., 22:203-254, 1972.

[40] V. V. Frolov et al. Electroproduction of the $\Delta(1232)$ Resonance at High Momentum Transfer. Phys. Rev. Lett., 82:45-48, 1999.

[41] Valery Frolov. Electroproduction of the $\Delta$ (1232) Resonance at High Momentum Transfer. PhD thesis, Rensselaer Polytechnic Institute, 1998.

[42] T. Fujii et al. Measurement of $\pi^{-} / \pi^{+}$Ratios in Photoproduction from Deuterium - the Dip Test of an Isotensor Current. Phys. Rev. Lett., 28:1672-1675, 1972.

[43] D. Gaskell. How We Measure Electronic Dead Time. 10 pages, unpublished, 2003.

[44] Murray Gell-Mann. The Eightfold Way: A Theory of Strong Interaction Symmetry. California Institute of Technology CTSL-20, unpublished. 
[45] S. Godfrey and Nathan Isgur. Mesons in a Relativized Quark Model with Chromodynamics. Phys. Rev., D32:189-231, 1985.

[46] Dan Green. The Physics of Particle Detectors. Cambridge University Press, New York, 2000.

[47] David J. Gross and Frank Wilczek. Ultraviolet Behavior of Non-Abelian Gauge Theories. Phys. Rev. Lett., 30(26):1343-1345, 1973.

[48] A. J. G. Hey. Quarks and Symmetries. In A. Donnachie and G. Shaw, editors, Electromagnetic Interactions of Hadrons, volume 1, pages 1-72. Plenum Press, New York, 1978.

[49] Nathan Isgur and Gabriel Karl. P Wave Baryons in the Quark Model. Phys. Rev., D18:4187, 1978.

[50] Nathan Isgur, Gabriel Karl, and Roman Koniuk. D Waves in the Nucleon: A Test of Color Magnetism. Phys. Rev., D25:2394, 1982.

[51] F. James, R. Cousins, and G. Cowan. Statistics: in Review of Particle Physics (RPP 2004). Phys. Lett., B592:279-288, 2004.

[52] H. F. Jones and M. D. Scadron. Multipole $\gamma N \Delta$ Form-Factors and Resonant Photoproduction and Electroproduction. Ann. Phys., 81:1-14, 1973.

[53] M. K. Jones. Detector studies. private communication, 2007.

[54] M. K. Jones. Reference for pp Collision Cross Section. private communication, 2007.

[55] K. Joo et al. $Q^{2}$ Dependence of Quadrupole Strength in the $\gamma^{*} p \rightarrow \Delta(1232) \rightarrow$ $p \pi^{0}$ Transition. Phys. Rev. Lett., 88:122001, 2002.

[56] S. S. Kamalov, D. Drechsel, O. Hanstein, L. Tiator, and S. N. Yang. Unitary Isobar (MAID) and Dynamical Models for Pion Electroproduction. Nucl. Phys., A684:321-323, 2001. 
[57] J. J. Kelly et al. Recoil Polarization for Delta Excitation in Pion Electroproduction. Phys. Rev. Lett., 95:102001, 2005.

[58] Peter E. Kloeden and Eckhard Platen. Numerical Solution of Stochastic Differential Equations. Springer, New York, 1999.

[59] Christoph W. Leemann. The Continuous Electron Beam Accelerator Facility: CEBAF at the Jefferson Laboratory. Annu. Rev. Nucl. Part. Sci., 51:413-450, 2001.

[60] G. Peter Lepage and Stanley J. Brodsky. Exclusive Processes in Perturbative Quantum Chromodynamics. Phys. Rev., D22:2157, 1980.

[61] Rui Li and Ya. S. Derbenev. Canonical Formulations and Cancellation Effect in Electrodynamics of Relativistic Beams on a Curved Trajectory. 45 pages, unpublished, 2003.

[62] D. H. Lyth. Exclusive Electroproduction Processes. In A. Donnachie and G. Shaw, editors, Electromagnetic Interactions of Hadrons, volume 1, pages 159-217. Plenum Press, New York, 1978.

[63] F. Mandl and G. Shaw. Quantum Field Theory. Wiley, New York, 1993.

[64] Jon Mathews. Leptonic Decay of the $\Omega$. Phys. Rev., 137:B444-B447, 1965.

[65] L. C. Maximon and J. A. Tjon. Radiative Corrections to Electron Proton Scattering. Phys. Rev., C62:054320, 2000.

[66] David Meekins. Hall C Target Configuration. 3 pages, unpublished, 2003.

[67] Stanimir A. Metchev and Jonathan E. Grindlay. A Two-Dimensional Kolmogorov-Smirnov Test for Crowded Field Source Detection: ROSAT Sources in NGC 6397. Mon. Not. Roy. Astron. Soc., 335:73, 2002.

[68] Luke W. Mo and Yung-Su Tsai. Radiative Corrections to Elastic and Inelastic e p and $\mu$ p Scattering. Rev. Mod. Phys., 41:205-235, 1969. 
[69] R. G. Moorhouse. Exclusive Electroproduction Processes. In A. Donnachie and G. Shaw, editors, Electromagnetic Interactions of Hadrons, volume 1, pages 83142. Plenum Press, New York, 1978.

[70] P. Nyborg. Multiparticle Phase-Space in Terms of Invariant Momentum Transfers, Invariant Masses and Angles. Nuovo Cim., A65:544-577, 1970.

[71] Susumu Okubo. Note on Unitary Symmetry in Strong Interactions. Prog. Theor. Phys., 27:949-966, 1962.

[72] Susumu Okubo. Note on Unitary Symmetry in Strong Interactions II: Excited States of Baryons. Prog. Theor. Phys., 28:24-32, 1962.

[73] Vladimir Pascalutsa, Marc Vanderhaeghen, and Shin Nan Yang. Electromagnetic Excitation of the $\Delta(1232)$ Resonance. Phys. Rept., 437:125-232, 2007.

[74] Michael E. Peskin and Daniel V. Schroeder. An Introduction to Quantum Field Theory. Westview APB, USA, 1995.

[75] Hartmut Pilkuhn. The Interactions of Hadrons. North-Holland Publishing, Amsterdam, 1967.

[76] H. David Politzer. Reliable Perturbative Results for Strong Interactions? Phys. Rev. Lett., 30(26):1346, 1973.

[77] Th. Pospischil et al. Measurement of the Recoil Polarization in the $p\left(\vec{e}, \vec{e}^{\prime}\right) \pi^{0}$ Reaction at the $\Delta(1232)$ Resonance. Phys. Rev. Lett., 86:2959-2962, 2001.

[78] W. Rarita and Julian S. Schwinger. On a Theory of Particles With Half Integral Spin. Phys. Rev., 60:61, 1941.

[79] M.N. Rosenbluth. High Energy Elastic Scattering of Electrons on Protons. Phys. Rev., 79:615-619, 1950.

[80] V. Rossi et al. Analysis of the Reaction $\gamma n \rightarrow p \pi^{-}$in the First and Second Resonance Regions. Nuovo Cim., A13:59-89, 1973.

[81] J. J. Sakurai. Advanced Quantum Mechanics. Addison Wesley, New York, 1967. 
[82] A. M. Sandorfi et al. The E2/M1 ratio in Delta Photoproduction. Nucl. Phys., A629:171c-179c, 1998.

[83] N. F. Sparveris et al. Investigation of the Conjectured Nucleon Deformation at Low Momentum Transfer. Phys. Rev. Lett., 94:022003, 2005.

[84] N. F. Sparveris et al. Determination of Quadrupole Strengths in the $\gamma^{*} p \rightarrow$ $\Delta(1232)$ Transition at $Q^{2}=0.20-(\mathrm{GeV} / \mathrm{c})^{2}$. Phys. Lett., B651:102-107, 2007.

[85] S. Stave et al. Lowest $Q^{2}$ Measurement of the $\gamma^{*} p \rightarrow \Delta$ reaction: Probing the Pionic Contribution. Eur. Phys. J., A30:471-476, 2006.

[86] A. Thomas and W. Weise. The Structure of the Nucleon. Wiley-VCH, New York, 2001.

[87] M. Ungaro et al. Measurement of the $N \rightarrow \Delta(1232)^{+}$Transition at high Momentum Transfer by $\pi^{0}$ Electroproduction. Phys. Rev. Lett., 97:112003, 2006.

[88] K. Unser. A Toroidal DC Beam Current Transformer With High Resolution. IEEE Trans. Nuc. Sci., NS-28, 1981.

[89] Mark Vanderhaeghen. Theoretical Issues. In Shapes of Hadrons, pages 25-47. American Institute of Physics, 2006.

[90] G. Von Holtey, G. Knop, H. Stein, J. Stuempfig, and H. Wahlen. The $\pi^{-} / \pi^{+}$ Ratio of Photoproduction on Deuterium for Energies Between 240 and 400 MeV. Phys. Lett., B40:589-592, 1972.

[91] John Dirk Walecka. Theoretical Nuclear and Subnuclear Physics. Oxford, New York, 1995.

[92] John Dirk Walecka. Electron Scattering for Nuclear and Nucleon Structure. Cambridge, Cambridge UK, 2001.

[93] W. M. Yao et al. Review of particle physics. J. Phys., G33:1-1232, 2006. 
[94] D. R. Yennie and H. Suura. Higher Order Radiative Corrections to Electron Scattering. Phys. Rev., 105:1378, 1957.

[95] S. Youssef and R. Cousins. Monte Carlo Techniques: in Review of Particle Physics (RPP 2004). Phys. Lett., B592:289-293, 2004. 


\section{APPENDIX A \\ Leptonic Currents and Couplings}

\section{A.1 Dirac Bilinears}

In order to characterize leptons in the context of quantum field theory it is necessary to construct generalized currents with the proper Lorentz structure. For spin- $\frac{1}{2}$ Dirac particles the most fundamental mathematical objects used to build the Dirac equation are the gamma matrices. It is well known that in order to have a consistent theory of fermions we must be able to construct currents with the proper Lorentz properties yet still behave as half integral spin particles are expected to. In short, one needs a spin- $\frac{1}{2}$ representation of the Lorentz group. The way to construct such a representation is to use matrix objects which satisfy the Clifford algebra.

$$
\left\{\gamma^{\mu}, \gamma^{\nu}\right\}=2 \eta^{\mu \nu}
$$

As the Greek indices imply the objects $\gamma^{\mu}$ behave like Lorentz vectors. Because of this property it is fairly straightforward to construct quantum objects which involve leptonic fields and also have some specified space-time structure. One should realize, however, that the normal object $\psi^{\dagger} \psi$ is not a scalar with respect to space-time structure. Due to the relativistic construction of the theory one must use the object $\psi^{\dagger} \gamma^{0} \psi$ in its stead. For compactness of notation this leads to the following definition of the Pauli conjugate.

$$
\bar{\psi} \equiv \psi^{\dagger} \gamma^{0}
$$

Definition of two more fundamental objects used in spinor representations will allow one to construct currents with any Lorentz structure desired. The first basic object is the natural tensor generalization of the vector objects $\gamma^{\mu}$.

$$
\sigma^{\mu \nu} \equiv\left[\gamma^{\mu}, \gamma^{\nu}\right]
$$


It is also extremely useful to define a fifth gamma matrix which will end up being part of the parity projection operator ${ }^{2}$.

$$
\gamma^{5} \equiv i \gamma^{0} \gamma^{1} \gamma^{2} \gamma^{3}
$$

With all the previous definitions it is easy to define various current objects with given Lorentz properties. There are 16 independent quantities which will emerge.

\begin{tabular}{|c|c|c|}
\hline Lorentz Properties & Form & Independent Components \\
\hline scalar & $\psi \psi$ & 1 \\
vector & $\bar{\psi} \gamma^{\mu} \psi$ & 4 \\
tensor & $\bar{\psi} \sigma^{\mu \nu} \psi$ & 6 \\
pseudoscalar & $\bar{\psi} \gamma^{5} \psi$ & 1 \\
pseudovector & $\bar{\psi} \gamma^{5} \gamma^{\mu} \psi$ & 4 \\
\hline
\end{tabular}

Table A.1: Dirac bilinears.

One can easily verify the Lorentz properties of all the above currents. Notice that the addition of the $\gamma^{5}$ matrix in the definitions cause the object to behave oppositely under parity transformations.

\section{A.2 Dirac Equation and Lagrangian}

To understand the spin- $\frac{1}{2}$ asymptotic spinors one should understand the free Dirac equation. The simplest way to write the equation is in the momentum space representation (Fourier space).

$$
(\not p-m) \psi=0
$$

The notation $\not p$ is simply shorthand for $\gamma^{\mu} p_{\mu}$ where the $\gamma^{\mu}$ are the gamma matrices mentioned in Section A.1. The equation is made far simpler in the rest frame of the particle.

$$
\left(\gamma^{0} p_{0}-m\right) \psi=0
$$

\footnotetext{
${ }^{2}$ In d-dimensions this is the $d+1^{s t}$ gamma matrix but it may have more peculiar properties than one is used to. For example for $d=2$ the matrix $\gamma^{\mu} \gamma^{5}$ is not independent of $\gamma^{\mu}$ itself [74].
} 
One can easily see that there are four independent solutions to the above matrix equation in the rest frame of the particle. These correspond to electrons and positrons of spin up or down. Another important implication of the coordinate space Dirac equation (or properly its conjugate equation) can be easily derived.

$$
\bar{\psi}(\not p+m)=0
$$

Finally, it is important to note that these equations are simply Fourier space versions of the field equations resulting from the free Dirac Lagrangian density.

$$
\mathcal{L}\left[\psi, \partial_{\mu} \psi\right]=\bar{\psi}(x)(i \not \partial-m) \psi(x)
$$

\section{A.3 Spin- $\frac{3}{2}$}

The previous sections dealt with harmonizing the Lorentz transformation properties with the transformation properties for a quantum mechanical spin- $\frac{1}{2}$ field. When dealing with fundamental spin- $\frac{3}{2}$ fields, or at least fields which are allowed to be present in asymptotic states one must find a new representation of the Lorentz group. This representation was obtained as a specific case of a general construction for "spinor" objects of any half-integral spin by Pauli and Fierz [38] and was studied even earlier by Dirac [27]. Later, the spin- $\frac{3}{2}$ version was given in the compact form used here by Rarita and Schwinger [78].

As one can imagine, the simplest way to form a representation would be to basically "glue" together some combination of spin- $\frac{1}{2}$ and spin-1 objects. Indeed, the spinor object for a spin- $\frac{3}{2}$ field carries a Lorentz index along with a spinor index. Assuming all spinor indices are suppressed, one may write the following "merger" of the Dirac and Proca equations for a field of mass $m$.

$$
\left(\gamma^{\tau} \partial_{\tau}+m\right) \psi_{\mu}=0
$$

The field $\psi_{\mu}$ is the spin- $\frac{3}{2}$ field and has an implicit spinor index. The same symbol $\psi$ is used for this field since its Lorentz index will distinguish it from its spin- $\frac{1}{2}$ 
counterpart. The newly defined field also obeys the following equation:

$$
\gamma^{\mu} \psi_{\mu}=0
$$

It is interesting to note, that these equations can be obtained from a Lagrangian. The most natural way to write this Lagrangian (with the expectation of coupling to electromagnetic fields) has been reported by Rarita and Schwinger over sixty years ago [78].

$$
\mathcal{L}=\bar{\psi}_{\mu}\left(\gamma^{\tau} \partial_{\tau}+m\right) \psi^{\mu}-\frac{1}{3} \bar{\psi}_{\mu}\left(\gamma^{\mu} \partial^{\nu}+\gamma^{\nu} \partial^{\mu}\right) \psi_{\nu}+\frac{1}{3} \bar{\psi}_{\mu} \gamma^{\mu}\left(\gamma^{\tau} \partial_{\tau}-m\right) \gamma^{\nu} \psi_{\nu}
$$

Finally, the electromagnetic current which will couple to the photon field can be written out straightforwardly.

$$
J^{\mu}=\psi_{\nu} \gamma^{\mu} \psi^{\nu}
$$




\section{APPENDIX B \\ Lengthy Derivations}

\section{B.1 Inclusive Lepton Scattering}

One can write the unpolarized cross section for the inclusive process straightforwardly. Simply using the phase space in conjunction with the one photon exchange amplitudes defined in Section 1.3 the cross section is the following:

$$
\sigma_{i n}=\frac{e^{4}}{\rho_{1} \rho_{2} v_{\text {rel }}} \int \frac{d^{3} k^{\prime}}{(2 \pi)^{3} 2 E^{\prime}} \sum_{X} \int \prod_{i=1}^{N_{X}}\left(\frac{d^{3} p_{i}}{(2 \pi)^{3} 2 E_{p_{i}}}\right) \frac{1}{4} \sum_{\text {spins }} \tilde{\mathcal{M}}_{i n}^{\star} \tilde{\mathcal{M}}_{\text {in }}
$$

In Eqn. B. $1 \rho_{1}$ is the density of the incoming particle (its plane wave normalization) and $\rho_{2}$ is that of the target particle. Furthermore, the parameter $v_{r e l}$ is the relative velocity of the two particle initial system. Working in the lab frame as we are and assuming the lepton is effectively massless one has that $v_{r e l}=1$, that is, the speed of light. It should also be mentioned that in the interest of saving space the following definition has been used.

$$
\tilde{\mathcal{M}}_{i n}^{\star} \tilde{\mathcal{M}}_{i n} \equiv \frac{1}{e^{4}}(2 \pi)^{4} \delta^{4}\left(q+p-\sum_{i} p_{i}\right) \mathcal{M}_{i n}^{\star} \mathcal{M}_{i n}
$$

The differential cross section can be simply parameterized by using kinematic definitions for the lepton integration and using the high energy limit where $m_{e}$ can be neglected.

$$
\sigma_{i n}=\frac{e^{4}}{\rho_{1} \rho_{2} v_{r e l}} \int \frac{E^{\prime} d E^{\prime} d \Omega_{e}}{2(2 \pi)^{3}} \sum_{X} \int \prod_{i=1}^{N_{X}}\left(\frac{d^{3} p_{i}}{(2 \pi)^{3} 2 E_{p_{i}}}\right) \frac{1}{4} \sum_{\text {spins }} \tilde{\mathcal{M}}_{i n}^{\star} \tilde{\mathcal{M}}_{i n}
$$


It is easy to get the differential cross section for electron scattering from this expression.

$$
\frac{d^{2} \sigma_{i n}}{d \Omega_{e} d E^{\prime}}=\frac{\alpha^{2}}{4 \pi M} \frac{E^{\prime}}{E} \sum_{X} \int \prod_{i=1}^{N_{X}}\left(\frac{d^{3} p_{i}}{(2 \pi)^{3} 2 E_{p_{i}}}\right) \frac{1}{4} \sum_{\text {spins }} \tilde{\mathcal{M}}_{i n}^{\star} \tilde{\mathcal{M}}_{i n}
$$

The symbol $M$ is the mass of the struck system. Using this formulation one can then write the cross section in an equivalent way which separates the leptonic and hadronic contributions.

$$
\frac{d^{2} \sigma_{i n}}{d \Omega_{e} d E^{\prime}}=\frac{\alpha^{2}}{Q^{4}} \frac{E^{\prime}}{E} L_{\mu \nu} W^{\mu \nu}
$$

It should be noted that the definition $Q^{2} \equiv-q^{2}$ has been made simply for the satisfaction of working with positive numbers. As a consequence of this form of the differential cross section one must define the leptonic tensor $L_{\mu \nu}$ and the hadronic tensor $W^{\mu \nu}$ as follows.

$$
\begin{aligned}
& L_{\mu \nu} \equiv \frac{1}{2} \sum_{\text {spins }}\left\langle e\left|J^{\sigma}\right| e^{\prime}\right\rangle\left\langle e^{\prime}\left|J^{\tau}\right| e\right\rangle \eta_{\sigma \mu} \eta_{\tau \nu} \\
& W^{\mu \nu} \equiv \frac{1}{4 \pi M} \frac{1}{2} \sum_{\text {spins }} \sum_{X} \int \prod_{i=1}^{N_{x}} \frac{d^{3} p_{i}}{(2 \pi)^{3} 2 E_{p_{i}}} \\
& \quad \times(2 \pi)^{4} \delta^{4}\left(q+p-\sum_{i} p_{i}\right)\left\langle p\left|J_{\text {had }}^{\mu}\right| X\right\rangle\left\langle X\left|J_{\text {had }}^{\nu}\right| p\right\rangle
\end{aligned}
$$

It should be clear that these are the unpolarized versions of the leptonic and hadronic tensors. If it is necessary to consider the polarized versions the spin sums (and averaging factors) can simply be omitted. The leptonic tensor can always be evaluated explicitly and unambiguously, however, the hadronic tensor requires a detailed theoretical calculation involving QCD or a parameterization with unknown functions. The leptonic tensor can quickly be boiled down to an expression involving only traces of the Dirac matrices, which are well known ${ }^{3}$. In the present situation this

\footnotetext{
${ }^{3}$ In what follows and everywhere in this work the convention of [74] will be used where $\operatorname{tr}(O)$ represents a trace over Dirac indices. The same notation will be used for traces over matrices. The context should clearly separate these situations
} 
computation can be done immediately for the unpolarized case.

$$
L^{\mu \nu}=\frac{1}{2} \operatorname{tr}\left(\gamma^{\mu}\left(\not k^{\prime}+m_{e}\right) \gamma^{\nu}\left(\not k+m_{e}\right)\right)
$$

Furthermore, in any situation at reasonably high energy the electron mass terms can be neglected and a further simplification occurs.

$$
\begin{aligned}
L^{\mu \nu} & =\frac{1}{2} k_{\sigma}^{\prime} k_{\tau} \operatorname{tr}\left(\gamma^{\mu} \gamma^{\sigma} \gamma^{\nu} \gamma^{\tau}\right) \\
L^{\mu \nu} & =2\left(k^{\prime \mu} k^{\nu}+k^{\mu} k^{\prime \nu}-\left(k^{\prime} \cdot k\right) \eta^{\mu \nu}\right)
\end{aligned}
$$

Unfortunately the hadronic tensor does not lend so well to explicit evaluation because of the non-perturbative nature of QCD. One can, however, put the hadronic tensor into a much cleaner form which makes some non-perturbative operator analysis methods conceptually easier to visualize. First one should note that the overall momentum conserving delta function which appears in Eqn. B.6 can be cast in a simple form.

$$
(2 \pi)^{4} \delta^{4}\left(q+p-\sum_{i} p_{i}\right)=\int d^{4} x \exp \left(q+p-\sum_{i} p_{i}\right) \cdot x
$$

This relation can be combined with Eqn. B.6 to yield a more compact expression if one remembers some simple facts about the total 4-momentum operator $\hat{P}^{\mu}$. Obviously if one has a state that is an eigenstate of momentum there is the relation $e^{\hat{P} \cdot x}|p\rangle=e^{p \cdot x}|p\rangle$. Further, one can write a Hilbert space operator which is a function of spacetime in terms of the same operator evaluated at the zero spacetime point as thus: $e^{i \hat{P} \cdot x} \mathcal{O}(0) e^{-i \hat{P} \cdot x}=\mathcal{O}(x)$. In this sense, the 4-momentum operator retains the function of a "translation" operator in spacetime just as its 3-dimensional counterpart was the spacial "translation" operator in ordinary quantum mechanics. Making use of these computational facts one can write the hadronic tensor of Eqn. B.6 in a 
more transparent form.

$$
\begin{aligned}
W^{\mu \nu}=\frac{1}{4 \pi M} \frac{1}{2} \sum_{\text {spins }} \sum_{X} & \int \prod_{i=1}^{N_{X}} \frac{d^{3} p_{i}}{(2 \pi)^{3} 2 E_{p_{i}}} \int d^{4} x e^{i q \cdot x} \\
& \times\left\langle p\left|e^{i \hat{P} \cdot x} J_{\text {had }}^{\mu}(0) e^{-i \hat{P} \cdot x}\right| X\right\rangle\left\langle X\left|J_{\text {had }}^{\nu}(0)\right| p\right\rangle
\end{aligned}
$$

It is easy to see that the sum over all possible final hadronic states allows one to use the completeness relation. Strictly speaking, this sum must run over all states of particles in order to use the completeness relation, however, in the context of this matrix element the kinematically forbidden states will come with delta functions which cannot be satisfied. Therefore, it is enough to sum over only allowed states in this context.

$$
\sum_{X} \int \prod_{i=1}^{N_{X}} \frac{d^{3} p_{i}}{(2 \pi)^{3} 2 E_{p_{i}}}|X\rangle\langle X| \simeq \mathbb{I}
$$

The symbol II represents the identity operator. With this development the hadronic tensor is written in the most straightforward way allowable. This form in terms of operators will lend directly to some operator methods, including the operator product expansion (OPE) and calculation in lattice QCD [86].

$$
W^{\mu \nu}=\frac{1}{4 \pi M} \frac{1}{2} \sum_{\text {spins }} \int d^{4} x e^{i q \cdot x}\left\langle p\left|\left[J_{\text {had }}^{\mu}(x), J_{\text {had }}^{\nu}(0)\right]\right| p\right\rangle
$$

Note that the presence of the commutator in Eqn. B.12 gives a symmetric appearance and is conceptually more pleasing, although only one of the terms will contribute since the term with the ordering $J_{\text {had }}^{\nu}(0) J_{\text {had }}^{\mu}(x)$ will give rise to a delta function which cannot be satisfied kinematically.

\section{B.2 Electromagnetic Transition}

To make a solid connection with standard quantum mechanics of atomic states and their interaction with a quantized radiation field one can resort to a multipole expansion. One can then make use of the rotational properties of matrix elements (via the Wigner-Eckart theorem) to specify transitions between definite states of angular momentum $\left|J_{i} M_{i}\right\rangle$ and $\left|J_{f} M_{f}\right\rangle$. The sacrifice which one makes by using 
electromagnetic multipole amplitudes is manifest Lorentz invariance. This lack of Lorentz invariance of the interesting amplitudes will not be much of a problem since they will only be utilized in one frame, the rest frame of the produced state of definite angular momentum $\left|J_{f} M_{f}\right\rangle$. Figure B.1 shows a schematic of the electromagnetic excitation between states of definite total angular momentum. Though this sort of

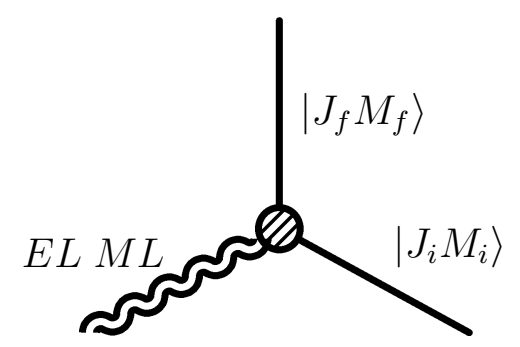

Figure B.1: Radiative transition in multipole basis.

excitation is what is typically observed in atomic physics and any photo-excitation processes in particle physics, it is not the most general which can occur. If one uses electroproduction to create "virtual" radiation a new type of multipole will appear in the transition amplitude, namely the Coulomb multipoles CL as can be seen in Fig. B.2. To begin to parameterize this process one should simply observe

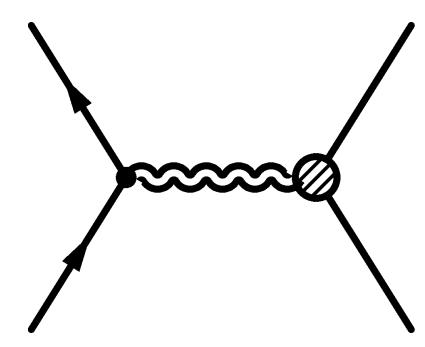

Figure B.2: Generalized electromagnetic transition in multipole basis.

the interaction Hamiltonian (density) of QED.

$$
\mathcal{H}_{I}^{Q E D}(x)=-e A_{\mu}(x) \bar{\psi}(x) \gamma^{\mu} \psi(x)-e_{h} A_{\mu}(x) J_{\text {had }}^{\mu}(x)
$$


The field $A^{\mu}(x)$ is the electromagnetic vector potential and $\psi(x)$ represents a lepton field of charge $e$. The symbol $J_{\text {had }}^{\mu}$ in Eqn. B.13 is the hadronic electromagnetic current operator while $e_{h}$ is the charge of the initial hadronic state. So long as $\frac{e}{e_{h}}$ is of order unity this interaction can be treated perturbatively in powers of $e^{4}$.

Though Eqn. B.13 is an easy way to see the full electromagnetic interaction in a natural covariant setting, it is not the most useful for multipole decompositions which are not Lorentz invariant. In this context, the most useful gauge for the quantization of the QED Hamiltonian is the Coulomb gauge, $\nabla \cdot \mathbf{A}=0$. This gauge will naturally separate the Coulomb multipoles (scalar) from the electric and magnetic (transverse) ones [91]. Taking a while to think, one can see the Hamiltonian (density) B.13 is correct but somehow seems not to easily correspond to the normal classical type Hamiltonian one has for electromagnetism with sources ${ }^{5}$.

$$
H^{C L}=\int d^{3} \mathbf{x}\left(|\mathbf{E}|^{2}+|\mathbf{B}|^{2}+\tilde{\mathbf{J}}_{e m} \cdot \mathbf{A}\right)
$$

Equation B.14 represents all the energies present in a classical collection of sources and radiation except for the energy of the "free" source. The interaction energy $\tilde{\mathbf{J}}_{e m} \cdot \mathbf{A}$ is included in Eqn. B.13 but it seems that the $A^{0}$ source terms like $-e \psi^{\dagger} \psi A^{0}$ are not present in B.14. The fact is that the terms are there but buried inside the energy of the electric field $\mathbf{E}$ because the sources are fully determined by the potential and $A^{0}$ cannot represent a separate degree of freedom according to Maxwell's law in the Coulomb gauge, $\nabla^{2} A^{0}(x)=\tilde{\rho}_{e m}(x)$. When using the covariant quantization and Eqn. B.13 it is useful to ignore this fact at the outset and then force it later by a constraint [81], but working in a non-covariant setting in the Coulomb gauge it is better to eliminate this degree of freedom immediately, before quantization. By a simple argument it can be shown that it is actually the longitudinal piece of $\mathbf{E}$ which contributes the mutual interaction energy of the free charges (and self interaction

\footnotetext{
${ }^{4}$ Or the corresponding fine structure constant.

${ }^{5}$ The symbol $\tilde{\mathbf{J}}_{\text {em }}$ represents the electric current density, in contrast to the quantum mechanical probability current which will be referred to without the tilde as $\mathbf{J}$. The same convention is used for $\tilde{\rho}_{e m}$ and $\rho$
} 
energy) and one can write B.15.

$$
H^{C L}=\int d^{3} \mathbf{x}\left(\left|\mathbf{E}_{T}\right|^{2}+|\mathbf{B}|^{2}+\tilde{\mathbf{J}}_{e m} \cdot \mathbf{A}+\left|\mathbf{E}_{L}\right|^{2}\right)
$$

If one writes $\mathbf{E}_{L}$ in terms of the matter densities, then the interaction Hamiltonian can be seen to be represented by the last two terms of Eqn. B.15. Noting that the first two terms would then correspond to the free electromagnetic field, one can omit this piece in the discussion because it serves only as a basis for quantization which is assumed to be done in some reasonable fashion. Before rewriting the interaction part of the Hamiltonian, some notation is necessary for the decomposition of the fourcurrents $J_{\text {had }}^{\mu}$ and $\bar{\psi} \gamma^{\mu} \psi$ into their non-covariant counterparts and for the passage to a quantum Hamiltonian.

$$
\begin{aligned}
J_{h a d}^{\mu} & \equiv\left(\rho_{h a d}, \mathbf{J}_{h a d}\right) \\
\bar{\psi} \gamma^{\mu} \psi & \equiv\left(\psi^{\dagger} \psi, \bar{\psi} \gamma \psi\right)
\end{aligned}
$$

Now, using Eqn. B.15 as a guide one can write the interaction Hamiltonian in terms of spacial integrals fairly simply. We now interpret all observables and fields as quantum mechanical operators. Quantizing in the Schrödinger picture is useful here, which means that the operators do not carry any time dependence. Everywhere else in this work it is understood that quantization is carried out in the interaction picture, with the "free field" time dependence carried in the operators.

$$
\begin{aligned}
H_{I}=e_{h} \int d^{3} \mathbf{x} & \mathbf{J}_{h a d}(\mathbf{x}) \cdot \mathbf{A}(\mathbf{x}) \\
& +e \int d^{3} \mathbf{x} \bar{\psi} \gamma \psi(\mathbf{x}) \cdot \mathbf{A}(\mathbf{x}) \\
& +\frac{e e_{h}}{4 \pi} \int d^{3} \mathbf{x} \int d^{3} \mathbf{x}^{\prime} \frac{\psi^{\dagger} \psi(\mathbf{x}) \rho_{\text {had }}\left(\mathbf{x}^{\prime}\right)}{\left|\mathbf{x}-\mathbf{x}^{\prime}\right|}
\end{aligned}
$$

The Greens function for the scalar potential has been used to write the last term in B.17. Now the problem of obtaining the excitation amplitudes for a transition from $\left|J_{i} M_{i}\right\rangle$ to a state $\left|J_{f} M_{f}\right\rangle$ are written by taking the matrix elements of the appropriate powers of $H_{I}$. It is obvious that the transitions described by Fig. B.1 
will be possible to lowest non-trivial order in $e_{h}$ while the more general transitions depicted in Fig.B.2 will only be possible to the next highest order, $\mathcal{O}\left(e e_{h}\right)$. Examination of the more general transitions from Fig. B.2 will be carried out here, the other transitions would be the subject of photoproduction analysis. Noting that the intermediate electromagnetic field in Fig. B.2 must be produced by another electrically interacting field which is taken here to be a lepton field, one can write the appropriate probability coefficients using Dirac's time-dependent perturbation theory.

$$
\begin{array}{r}
c^{(1)}(t)=-i \int_{0}^{t} d t^{\prime}\left\langle l_{f} ; J_{f} M_{f}\left|H_{I}\left(t^{\prime}\right)\right| l_{i} ; J_{i} M_{i}\right\rangle e^{i\left(E_{f}-E_{i}\right) t^{\prime}} \\
c^{(2)}(t)=-\sum_{n} \int_{0}^{t} d t^{\prime \prime} \int_{0}^{t^{\prime \prime}} d t^{\prime}\left\langle l_{f} ; J_{f} M_{f}\left|H_{I}\left(t^{\prime \prime}\right)\right| n\right\rangle e^{i\left(E_{f}-E_{n}\right) t^{\prime \prime}} \\
\times\left\langle n\left|H_{I}\left(t^{\prime}\right)\right| l_{i} ; J_{i} M_{i}\right\rangle e^{i\left(E_{n}-E_{i}\right) t^{\prime}}
\end{array}
$$

The interaction Hamiltonian does not depend on time explicitly so that the previous expressions can be simplified considerably by carrying out the time integrations.

$$
\begin{gathered}
c^{(1)}(t)=-i\left\langle l_{f} ; J_{f} M_{f}\left|H_{I}\right| l_{i} ; J_{i} M_{i}\right\rangle \int_{0}^{t} d t^{\prime} e^{i\left(E_{f}-E_{i}\right) t^{\prime}} \\
c^{(2)}(t)=-\left\langle l_{f} ; J_{f} M_{f}\left|H_{I} H_{I}\right| l_{i} ; J_{i} M_{i}\right\rangle \int_{0}^{t} d t^{\prime \prime} \int_{0}^{t^{\prime \prime}} d t^{\prime} e^{i\left(E_{f}-E_{n}\right) t^{\prime \prime}} e^{i\left(E_{n}-E_{i}\right) t^{\prime}}
\end{gathered}
$$

The seemingly complicated functions of time appearing at the end of Eqns.B.20 and B.21 will boil down to linear contributions in time multiplied by energy conserving delta functions upon squaring of the amplitudes. Since the usual $S$ matrix elements are generically related to these coefficients by:

$$
S=\lim _{t \rightarrow \infty} \frac{|c(t)|^{2}}{t}
$$

the linear time dependence will cancel and the portion of the $c(t)$ 's which will be important are the Dirac brackets.

Note that a part of the Dirac brackets we are interested in can be explicitly calculated using Quantum Electrodynamics. Carrying out this operation will leave matrix elements of the hadronic currents and charges which are the unknowns that 
essentially need to be measured. The process will then be boiled down to three complex quantities which will serve as the meeting ground for theory and experiment. Furthermore, since the explicit calculations depend only on the quantization of the lepton fields, the computations are quite independent from any particular gauge. Background information on the quantization of Dirac particles is given in AppendixA. The Coulomb piece of the matrix element can easily be written out explicitly to the order $\mathcal{O}\left(e e_{h}\right)$. Since it is typical that this would be called second order in the electromagnetic interaction a superscript (2) will accompany the matrix element even though it involves only one power of the interaction Hamiltonian.

$$
\begin{aligned}
\mathcal{M}_{c}^{(2)} & \equiv\left\langle l_{f} ; J_{f} M_{f}\left|H_{I}\right| l_{i} ; J_{i} M_{i}\right\rangle \\
& =\frac{e e_{h}}{4 \pi} \int d^{3} \mathbf{x}\left\langle l_{f}\left|\psi^{\dagger} \psi\right| l_{i}\right\rangle \int d^{3} \mathbf{x}^{\prime} \frac{\left\langle J_{f} M_{F}\left|\rho_{h a d}\left(\mathbf{x}^{\prime}\right)\right| J_{i} M_{i}\right\rangle}{\left|\mathbf{x}-\mathbf{x}^{\prime}\right|}
\end{aligned}
$$

Some manipulation of Eqn. B.23 shows a that there is a factor of the Fourier transform of the Coulomb potential.

$$
\begin{aligned}
\mathcal{M}_{c}^{(2)}=e e_{h} \int d^{3} \mathbf{x}^{\prime} u^{\dagger}\left(k^{\prime}, s^{\prime}\right) u(k, s)\left\langle J_{f} M_{F}\right| & \rho_{h a d}\left(\mathbf{x}^{\prime}\right)\left|J_{i} M_{i}\right\rangle e^{i \mathbf{q} \cdot \mathbf{x}^{\prime}} \\
\times & {\left[\int d^{3} \mathbf{x} \frac{e^{i \mathbf{q} \cdot\left(\mathbf{x}-\mathbf{x}^{\prime}\right)}}{4 \pi\left|\mathbf{x}-\mathbf{x}^{\prime}\right|}\right] }
\end{aligned}
$$

The term in brackets is simply the Fourier transform of the Coulomb potential and can be evaluated explicitly after a simple change of variables.

$$
\mathcal{M}_{c}^{(2)}=\frac{e e_{h}}{|\mathbf{q}|^{2}}\left\langle J_{f} M_{F}\left|\int d^{3} \mathbf{x} u_{f}^{\dagger}\left(k^{\prime}, s^{\prime}\right) u_{i}(k, s) \rho_{h a d}(\mathbf{x}) e^{i \mathbf{q} \cdot \mathbf{x}}\right| J_{i} M_{i}\right\rangle
$$

The variables $k, k^{\prime}, s$, and $s^{\prime}$ are the initial lepton 4-momentum, final lepton 4momentum, initial spin and final spin respectively. This is about as far as one can go explicitly before invoking some expansion techniques to separate the contributions to $\left\langle f\left|\rho_{\text {had }} e^{i q x}\right| i\right\rangle$. A similar tack can be used to evaluate the Dirac bracket which will contain the electric and magnetic contributions. This contribution will require 
two actions of the interaction Hamiltonian.

$$
\begin{aligned}
& \mathcal{M}_{e, m}^{(2)} \equiv\left\langle l_{f} ; J_{f} M_{f}\left|H_{I} H_{I}\right| l_{i} ; J_{i} M_{i}\right\rangle \\
&=e e_{h} \int d^{3} \mathbf{x} \int d^{3} \mathbf{x}^{\prime}\left\langle l_{f}\left|\psi^{\dagger} \gamma^{0} \gamma \psi(\mathbf{x}) \cdot \mathbf{A}(\mathbf{x})\right| l_{i}\right\rangle \\
& \quad \times\left\langle J_{f} M_{F}\left|\mathbf{J}_{h a d}\left(\mathbf{x}^{\prime}\right) \cdot \mathbf{A}\left(\mathbf{x}^{\prime}\right)\right| J_{i} M_{i}\right\rangle
\end{aligned}
$$

It is obvious that in Eqn. B.26 one must evaluate the factor $\left\langle 0\left|A^{i}(\mathbf{x}) A^{j}\left(\mathbf{x}^{\prime}\right)\right| 0\right\rangle$. This is essentially the Coulomb gauge analog of the Feynman propagator. Although evaluation of the mentioned factor is not especially difficult there is a simple way around it when working in the one photon exchange limit [24]. Since with one photon exchange the quantum character of the electromagnetic radiation does not come into play, one can replace the photon field operator $\mathbf{A}(\mathbf{x})$ with an external c-number field $\mathbf{A}^{\text {ext }}(\mathbf{x})$ which is sourced by $\left\langle f\left|\mathbf{J}_{\text {had }}(\mathbf{x})\right| i\right\rangle$.

$$
\mathcal{M}_{e, m}^{(2)}=\frac{e e_{h}}{|\mathbf{q}|^{2}}\left\langle J_{f} M_{F}\left|\int d^{3} \mathbf{x} u_{f}^{\dagger}\left(k^{\prime}, s^{\prime}\right) \gamma^{0} \gamma u_{i}(k, s) \cdot \mathbf{J}_{h a d}(\mathbf{x}) e^{i \mathbf{q} \cdot \mathbf{x}}\right| J_{i} M_{i}\right\rangle
$$

For brevity let $E$ stand for the magnitude of the Dirac spinor object above and let e be the unit vector in its direction.

$$
\mathcal{M}_{e, m}^{(2)}=\frac{e e_{h} E}{|\mathbf{q}|^{2}}\left\langle J_{f} M_{F}\left|\int d^{3} \mathbf{x} \mathbf{e} \cdot \mathbf{J}_{h a d}(\mathbf{x}) e^{i \mathbf{q} \cdot \mathbf{x}}\right| J_{i} M_{i}\right\rangle
$$

The expansion of the Dirac brackets in $\mathcal{M}_{c}^{(2)}$ and $\mathcal{M}_{e, m}^{(2)}$ in terms of tensor operators is a useful endeavor. A very elegant treatment has been given in several references $[92,24]$. Indeed, the expansion itself is not too difficult, Eqns. B.27 and B.25 already expand the current and charge matrix elements, but into the continuous Fourier basis. Any physics student knows that the infinite but denumerable set of spherical harmonics plus the set of spherical Bessel functions will do just as well for a continuous three dimensional scalar function. However this set has the advantage that the contributions of definite angular momentum are separated. Expanding the term $e^{i \mathbf{q} \cdot \mathbf{x}}$ will do to make this expansion. Here and in what follows all spins and angular momenta are understood to be quantized along the momentum transfer 
vector $\mathbf{q} \equiv \mathbf{k}-\mathbf{k}^{\prime}$ which defines the " $\mathrm{z}$ " axis.

$$
e^{i \mathbf{q} \cdot \mathbf{x}}=\sum_{J}(i)^{J} \sqrt{4 \pi(2 J+1)} j_{J}(q x) Y_{J 0}\left(\Omega_{x}\right)
$$

Where the magnitudes $|\mathbf{q}|$ and $|\mathbf{x}|$ have been abbreviated by $q$ and $x$ when in the argument of the spherical Bessel function $j_{J}(\cdot)$. The symbols $\Omega_{q}$ and $\Omega_{x}$ are the usual shorthand for the angles of the corresponding vectors. For the electric and magnetic components the expansion requires that the slightly more complex object $\mathbf{e} e^{i \mathbf{q} \cdot \mathbf{x}}$ be expanded $[92,24]$. It is most useful to use the helicity expansion for the unit vector e.

$$
\mathbf{e}=\sum_{\lambda} a_{\lambda} \mathbf{e}_{\lambda}
$$

Then one can use the angular expansion of the factor $e^{i \mathbf{q} \cdot \mathbf{x}}$ along with the transformation from the coupled basis to the basis of total angular momentum to get the desired expansion. As far as taking care of the angular part of this basis transformation one can simply use the vector spherical harmonics.

$$
\mathbf{Y}_{J J 1}^{M}\left(\Omega_{x}\right) \equiv \sum_{m m^{\prime}}\left(J m 1 m^{\prime} \mid J 1 J M\right) Y_{J m}\left(\Omega_{x}\right) \mathbf{e}_{m^{\prime}}
$$

The symbols $\left(j_{1} m_{1} j_{2} m_{2} \mid j_{1} j_{2} j m\right)$ are the Clebsh-Gordon coefficients as used by Edmonds [34]. The appropriate expansion can then be made using the polarization vectors $\mathbf{e}_{\lambda}$. The details of this expansion has been investigated [92] and is mostly an exercise in patience and knowledge of properties of the Clebsh-Gordon coefficients.

$$
\begin{aligned}
\mathbf{e}_{\lambda} e^{i \mathbf{q} \cdot \mathbf{x}}=-\sqrt{2 \pi} \sum_{J \geq 1}(i)^{J} \sqrt{2 J+1}[ & -\lambda j_{J}(q x) \mathbf{Y}_{J J 1}^{\lambda}\left(\Omega_{x}\right) \\
& \left.-\frac{1}{|\mathbf{q}|} \nabla \times\left(j_{J}(q x) \mathbf{Y}_{J J 1}^{\lambda}\left(\Omega_{x}\right)\right)\right]
\end{aligned}
$$

This expansion motivates the definition of several operators. The benefit of these definitions will not only be aesthetic but calculational. In particular one will find that the dependence of the matrix elements on the initial and final magnetic quantum numbers $M_{i}$ and $M_{f}$ is totally superficial and can be written explicitly without 
any knowledge of the hadronic dynamics.

$$
\begin{aligned}
\hat{M}_{J M}^{c}(|\mathbf{q}|) & \equiv \int d^{3} \mathbf{x} j_{J}(q x) Y_{J M}\left(\Omega_{x}\right) \rho_{h a d}(\mathbf{x}) \\
\hat{T}_{J M}^{e}(|\mathbf{q}|) & \equiv \frac{1}{|\mathbf{q}|} \int d^{3} \mathbf{x} \nabla \times\left[j_{J}(q x) \mathbf{Y}_{J J 1}^{M}\left(\Omega_{x}\right)\right] \cdot \mathbf{J}_{h a d}(\mathbf{x}) \\
\hat{T}_{J M}^{m}(|\mathbf{q}|) & \equiv \int d^{3} \mathbf{x} j_{J}(q x) \mathbf{Y}_{J J 1}^{M}\left(\Omega_{x}\right) \cdot \mathbf{J}_{\text {had }}(\mathbf{x})
\end{aligned}
$$

These are the Coulomb, Transverse electric and Transverse magnetic operators respectively. These operators enjoy many useful properties. The most important property that will be exploited is that they are all irreducible tensor operators (ITOs) and have definite rotational properties when taken between states of definite angular momentum [34]. The Coulomb excitation amplitude can be written in terms of matrix elements of these new operators.

$$
\mathcal{M}_{c}^{(2)}=\frac{e e_{h}}{|\mathbf{q}|^{2}} u^{\dagger}\left(k^{\prime}, s^{\prime}\right) u(k, s) \sum_{J}(i)^{J} \sqrt{4 \pi(2 J+1)}\left\langle J_{f} M_{f}\left|\hat{M}_{J 0}^{c}\right| J_{i} M_{i}\right\rangle
$$

The transverse amplitudes may also be written in terms of matrix elements of these special operators.

$$
\begin{aligned}
\mathcal{M}_{e, m}^{(2)}=-\frac{e e_{h} E}{|\mathbf{q}|^{2}} \sqrt{2 \pi} \sum_{\substack{\lambda= \pm J \geq 1}} a_{\lambda}(i)^{J} \sqrt{2 J+1}\left[\lambda\left\langle J_{f} M_{f}\left|\hat{T}_{J \lambda}^{m}\right| J_{i} M_{i}\right\rangle\right. & \\
& \left.+\left\langle J_{f} M_{f}\left|\hat{T}_{J \lambda}^{e}\right| J_{i} M_{i}\right\rangle\right]
\end{aligned}
$$

Equations B.34 and B.35 leave the functional dependence of the irreducible tensor operators implicit. The motivation for calling the operator $\hat{M}_{J M}^{c}$ a "Coulomb" operator is obviously due to the fact that it comes from the instantaneous interaction when QED is quantized in the Coulomb gauge (i.e. with Hamiltonian B.17). The motivation for the "electric" or "magnetic" specification of the transverse operators is due to the analog to electric and magnetic fields. Electric fields are vectors and magnetic fields are pseudovectors. The construction of each of the transverse transition operators is motivated, then, by the fact that the separation listed in Eqn. B.33 has each of the transverse operators with definite parity transformations 
[92].

$$
\begin{aligned}
\hat{\Pi} \hat{T}_{J M}^{e} \hat{\Pi}^{-1} & =(-1)^{J} \hat{T}_{J M}^{e} \\
\hat{\Pi} \hat{T}_{J M}^{m} \hat{\Pi}^{-1} & =(-1)^{J+1} \hat{T}_{J M}^{m}
\end{aligned}
$$

The operator $\hat{\Pi}$ represents the parity transformation. Equation B.36 gives the proper parity rules for the electric and magnetic dipole transitions $(J=1)$, which should have the same parity as the electric and magnetic fields themselves.

The interesting process in this work involves a $\Delta$ particle excitation from a proton target struck with an electron current ${ }^{6}$. The measurement is completely unpolarized so that neither the initial or final magnetic quantum number is observed or constrained in any way. This means that the cross sections produced should include sums over $M_{i}$ and $M_{f}$ in our notation. Some simple combinatorics shows that if this is the case one has $\left(2 J_{i}+1\right)\left(2 J_{f}+1\right)$ possible matrix elements for each ITO to parameterize the interaction.

There are several theorems in quantum mechanics whose genius is only matched by their simplicity. It is an honor for a young student to be able to recognize the ingenuity of his or her predecessors and an inspiration to try to construct ideas of the same utility. In this situation, of course, I am referring to the theorem of Wigner and Eckart. By the rotational properties of the previously constructed ITOs one can explicitly write the dependence on magnetic quantum numbers and even have a clear handle on all selection rules. For a general ITO we define the "double-barred" or "reduced" matrix element by the following equality [34, 92].

$$
\left\langle J_{f} M_{f}\left|\hat{O}_{J M}\right| J_{i} M_{i}\right\rangle=\frac{(-1)^{J_{i}-M_{i}}}{\sqrt{2 J+1}}\left(J_{f} M_{f} J_{i}-M_{i} \mid J_{f} J_{i} J M\right)\left\langle J_{f}|| \hat{O}_{J}|| J_{i}\right\rangle
$$

With this knowledge one can parameterize a $\Delta$ electro-excitation by the three most important reduced matrix elements restricting to at most a p-wave $\pi N$ final state

\footnotetext{
${ }^{6}$ The production of neutral pions is the process actually observed.
} 
as we will in this analysis (Chapter 10).

$$
\begin{aligned}
M 1 & \equiv\left\langle\frac{3}{2}\left\|\hat{T}_{1}^{m}\right\| \frac{1}{2}\right\rangle \\
E 2 & \equiv\left\langle\frac{3}{2}\left\|\hat{T}_{2}^{e}\right\| \frac{1}{2}\right\rangle \\
C 2 & \equiv\left\langle\frac{3}{2}\left\|\hat{M}_{2}^{c}\right\| \frac{1}{2}\right\rangle
\end{aligned}
$$

Note that these amplitudes do not interfere because they have different selection rules for an asymptotic $\Delta$ state and thus can be taken with any phase. Since in the current analysis one only measures outgoing neutral pions, there must be a way to link the angular distributions to these excitation amplitudes to get an indirect measure. This is exactly what will be done by using angular momentum conservation and parity conservation as constraints along with a resonance dominance assumption at the point where the invariant energy is equal to the $\Delta$ mass. 


\section{APPENDIX C Kinematical Conventions}

\section{C.1 Introduction}

Several variations on the electron scattering kinematics are treated throughout the text. It is useful to keep the kinematic definitions as consistent as possible. The purpose of the present appendix is to organize the kinematic definitions for several typical processes. Two specific frames of reference will be used frequently and therefore a general notation will be used for non-covariant variables in each of these frames. As an example take a 4 -vector $k$. The vector will be referred to as such in all frames so long as it is used with its full covariant structure as in the expression $k \cdot p$ or the expression $k^{\mu} p_{\mu}$, where $p$ is some other 4 -vector. However should one need to refer to the components of $k$ which are not covariant, a star will distinguish the center of mass frame from the lab frame. Therefore, $k^{0}$ will be the zeroth component of the 4 -vector in the lab and $k^{0 *}$ the corresponding quantity in the center of mass. Similarly a laboratory angle $\theta$ will be distinguished from $\theta^{*}$, its center of mass counterpart. Any other frame will be explicitly mentioned in the text should the need arise.

The angles defined in this thesis will be implicitly assumed to be consistent with the angular coordinate system in Fig. C.1. The polar angle is measured down from the " $\mathrm{z}$ " axis, the axis corresponding to $\theta=0$. The azimuthal angles are measured such that the " $\mathrm{x}$ " axis corresponds to the point $\theta=\frac{\pi}{2}$ and $\phi=0$. Further,

the "y" axis corresponds to $\theta=\frac{\pi}{2}$ and $\phi=\frac{\pi}{2}$. The azimuthal angle is taken be in the range $-\pi \leq \phi \leq \pi$.

\section{C.2 Elastic Scattering}

Elastic scattering is represented throughout the text by the following lab frame variables which have center of mass frame analogs according to the convention es- 


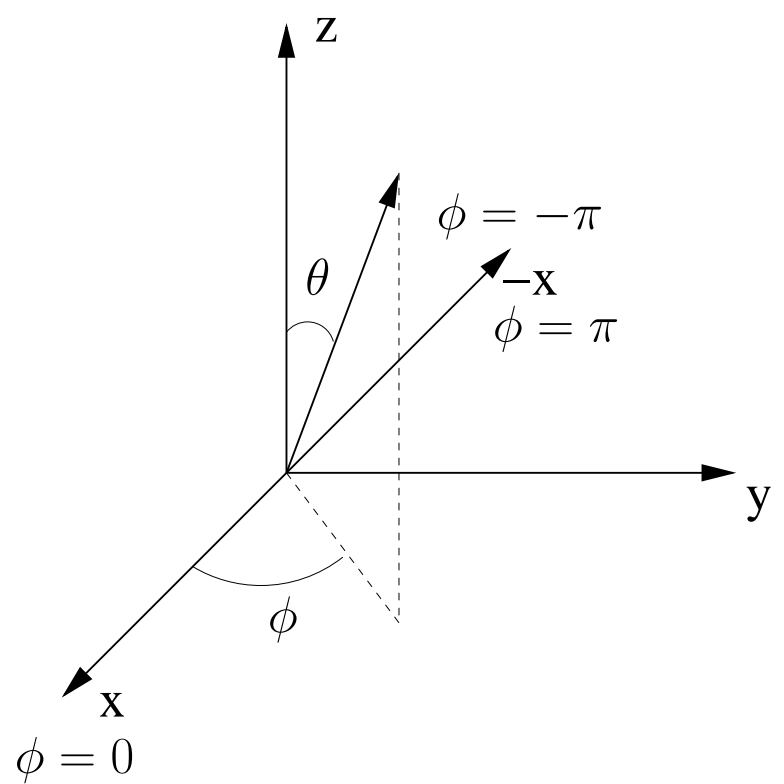

Figure C.1: Angular definitions.

poused in the current appendix.

$$
\begin{aligned}
k & ; \text { initial electron 4-momentum } \\
k^{\prime} ; & \text { final electron 4-momentum } \\
E & ; \text { initial electron energy } \\
E^{\prime} ; & \text { final electron energy } \\
p & ; \text { initial proton 4-momentum } \\
p^{\prime} & ; \text { final proton 4-momentum } \\
E_{p} & ; \text { initial proton energy } \\
E_{p}^{\prime} ; & \text { final proton energy } \\
\theta_{e} & ; \text { angle between the incident and scattered electron }
\end{aligned}
$$




\section{C.3 Pion Production}

Pion production is represented throughout the text by the following.

$k \quad$; initial electron 4-momentum

$k^{\prime} \quad$; final electron 4-momentum

$k_{\pi} \quad ; \quad$ pion 4-momentum

$E \quad ; \quad$ initial electron energy

$E^{\prime} \quad$; final electron energy

$p \quad ; \quad$ initial proton 4-momentum

$p^{\prime} \quad ; \quad$ final proton 4-momentum

$E_{p} \quad ; \quad$ initial proton energy

$E_{p}^{\prime} \quad ; \quad$ final proton energy

$\theta_{e} ;$ angle between the incident and scattered electron

$\theta$; azimuthal angle between the momentum transfer and the pion

$\phi$; polar angle of the pion with z-axis defined along the momentum transfer 


\section{C.4 Elastic Radiative}

Elastic radiative kinematics are represented throughout the text by the following.

$k \quad ; \quad$ initial electron 4-momentum

$k^{\prime} \quad$; final electron 4-momentum

$k_{\gamma} \quad ; \quad$ radiated photon 4 -momentum

$E \quad ; \quad$ initial electron energy

$E^{\prime} \quad$; final electron energy

$p \quad ; \quad$ initial proton 4-momentum

$p^{\prime} \quad ; \quad$ final proton 4-momentum

$E_{p} \quad ; \quad$ initial proton energy

$E_{p}^{\prime} \quad ; \quad$ final proton energy

$\theta_{e} ;$ angle between the incident and scattered electron

$\theta_{\gamma} ;$ azimuthal angle between the radiating particle and the radiated photon 


\section{C.5 Pion Production Radiative}

Pion production radiative kinematics are represented throughout the text by the following.

$k \quad$; initial electron 4-momentum

$k^{\prime} \quad$; final electron 4-momentum

$k_{\gamma} \quad ; \quad$ radiated photon 4 -momentum

$k_{\pi} ;$ pion 4-momentum

$E \quad ; \quad$ initial electron energy

$E^{\prime} \quad$; final electron energy

$p \quad ; \quad$ initial proton 4-momentum

$p^{\prime} \quad ; \quad$ final proton 4-momentum

$E_{p} \quad ; \quad$ initial proton energy

$E_{p}^{\prime} \quad ; \quad$ final proton energy

$\theta_{e} ;$ angle between the incident and scattered electron

$\theta_{\gamma} ;$ azimuthal angle between the radiating particle and the radiated photon

$\theta ;$ azimuthal angle between $\left(\mathbf{k}-\mathbf{k}^{\prime}\right)$ and the pion

$\phi \quad ; \quad$ polar angle of the pion the vector $\left(\mathbf{k}-\mathbf{k}^{\prime}\right)$ 


\section{APPENDIX D \\ Experimental Parameters}

\section{D.1 Data Overview}

Exclusive electroproduction data for the process $e p \rightarrow e^{\prime} p^{\prime} X$ was gathered in the spring of 2003 run period at Jefferson Lab. The data was taken using the facilities in Jefferson Lab's Hall C, including the HMS (high momentum spectrometer) and SOS (short orbit spectrometer). The HMS was used to measure the hadron (proton) properties while the SOS was used to measure the lepton (electron) properties. Though the magnetic spectrometers have a small acceptance compared to the acceptance of a $4 \pi$ detector, full coverage can be obtained in the center of mass variables $^{7}$ using a several HMS angle scans. The spectrometer settings for the experiment are listed in Tbls. D.1 and D.2. The beam energy was $5.5 \mathrm{GeV}$.

\begin{tabular}{|c|c|c|c|}
\hline SOS $\measuredangle$ & $p(\mathrm{GeV})$ & HMS Ł & $\operatorname{HMS} p(\mathrm{GeV})$ \\
\hline \multirow{5}{*}{$47.5^{\circ}$} & \multirow{5}{*}{1.71} & $19.5^{\circ}, 16.5^{\circ}, 13.5^{\circ}, 11.2^{\circ}$ & 4.5 \\
\hline & & $22.5^{o}, 19.5^{o}, 16.5^{o}, 13.5^{o}, 11.2^{o}$ & 3.7 \\
\hline & & $22.5^{o}, 19.5^{o}, 16.5^{o}, 13.5^{o}, 11.2^{o}$ & 3.1 \\
\hline & & $22.5^{o}, 19.5^{o}, 16.5^{o}, 13.5^{o}, 11.2^{o}$ & 2.6 \\
\hline & & $22.5^{o}, 19.5^{o}, 16.5^{o}, 13.5^{o}$ & 2.1 \\
\hline
\end{tabular}

Table D.1: Experimental spectrometer settings for $Q^{2}=6.36 \mathbf{G e V}^{2}$.

\begin{tabular}{|cc|c|c|}
\hline SOS $\measuredangle$ & SOS $p(\mathrm{GeV})$ & HMS $\measuredangle$ & HMS $p(\mathrm{GeV})$ \\
\hline \multirow{6}{*}{$69.9^{\circ}$} & & $11.2^{\circ}$ & 4.7 \\
& & $14.2^{\circ}$ & 4.5 \\
& & $11.2^{o}$ & 3.9 \\
& & $14.2^{o}, 11.2^{\circ}$ & 3.7 \\
& & $11.2^{\circ}$ & 3.2 \\
\hline
\end{tabular}

Table D.2: Experimental spectrometer settings for $Q^{2}=7.7 \mathrm{GeV}^{2}$.

\footnotetext{
${ }^{7}$ This fact is due to the somewhat large value of momentum transfer $Q^{2}$ and kinematic focusing in the transformation from lab to center of mass variables.
} 
It is useful to examine the overall results of the experiment to obtain intuition about backgrounds and cuts. The most natural distributions to look at to gain overall understanding are the invariant mass and missing mass distributions $(W(\mathrm{GeV})$ and $M M^{2}\left(\mathrm{GeV}^{2}\right)$ respectively). These distributions obviously show which mesons are being produced (and selected) along with which regions of invariant mass the meson events come from predominately. For example one can see a clear picture of the resonances under study from the invariant mass distribution. The missing mass distribution can be seen in Fig. D.1. It is clear that in some loose sense $\pi^{0}$ production corresponds to the $\Delta(1232)$ resonance and $\eta$ production corresponds to the $S_{11}(1535)$ resonance. From the picture it looks as if $\omega$ production also has a corresponding bump in the $\mathrm{W}$ distribution, although, it is difficult to say that this is a resonance since this region of $\mathrm{W}$ is near the edge of the experimental acceptance. It should be said that the $\Delta(1232)$ is by no means the only source of $\pi^{0}$ production, and correspondingly for the $\eta$ and $S_{11}(1535)$.
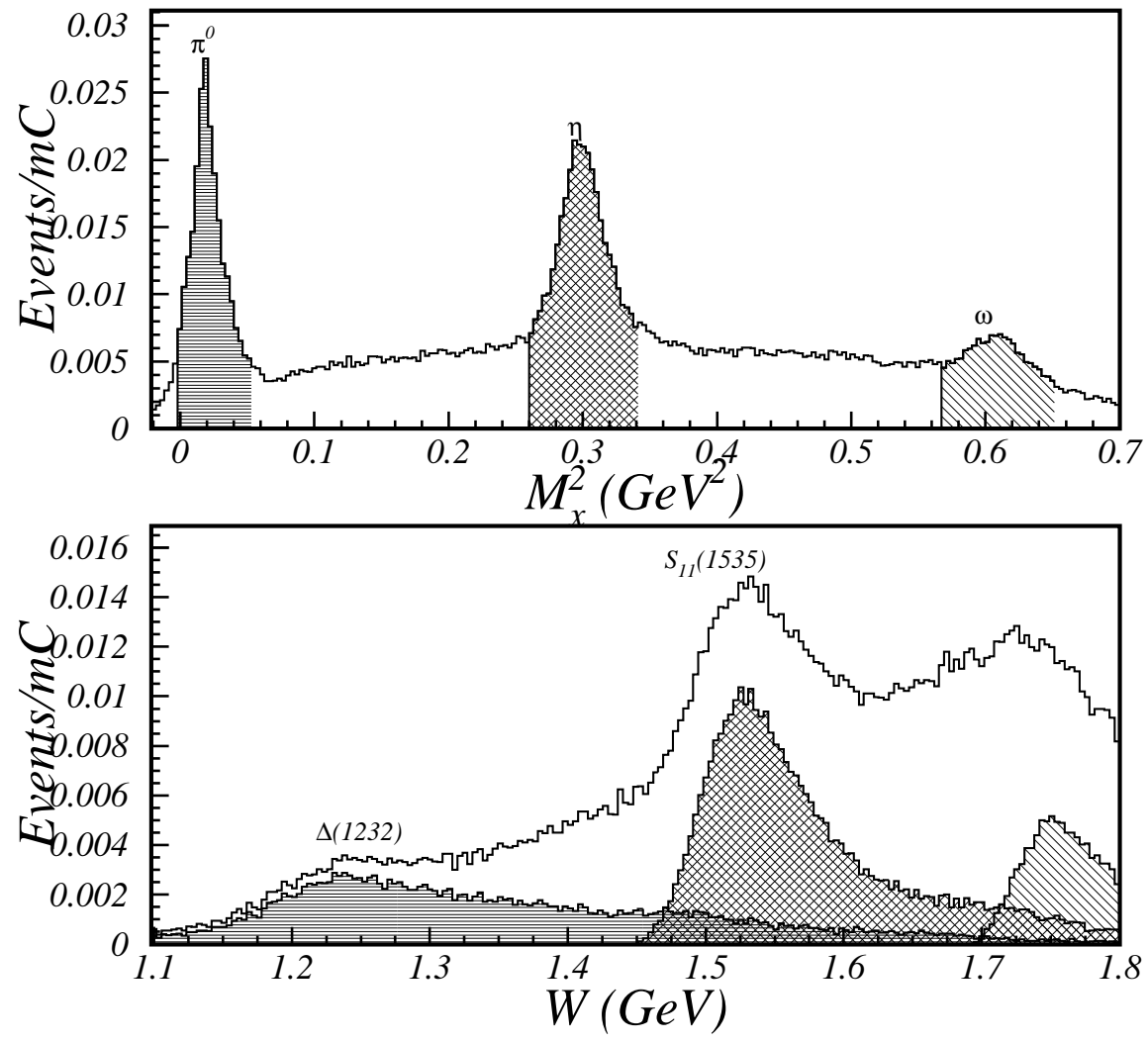

Figure D.1: Missing mass distribution mapped onto W distribution. 
One can also view this same data on a two dimensional plot to bring light to the correlation between invariant mass and missing mass. The elastic, $\pi^{0}, \eta$, and $\omega$

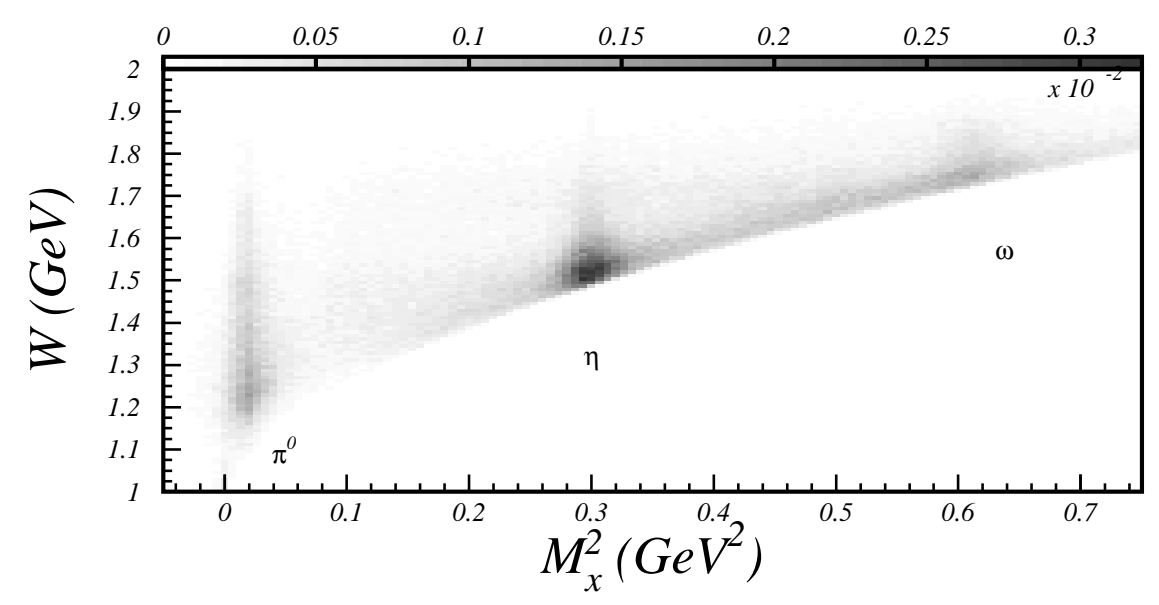

Figure D.2: Invariant mass vs. missing mass distribution.

peaks are clearly visible in Fig. D.2. The pion peak is clearly more spread out in invariant mass and undoubtedly includes a strong non-resonant background. The eta peak seems somewhat sharp in invariant mass and the $S_{11}(1535)$ seems as if it is rising above the background by a significant amount even at this high value of $Q^{2} \sim 6.3 \mathrm{GeV}^{2}$. The omega seems to come from a concentrated region of invariant mass but since the acceptance is dropping near that value of invariant mass it is difficult to associate a clear resonance with it. The $\omega$ peak may simply be reflecting the rise in meson production backgrounds at high invariant mass.

\section{D.2 Experimental Settings}

The settings listed in Section D.1 list clearly the physical settings for each Hall C configuration in the lab frame. The central momenta mentioned for each spectrometer setting are created by adjusting the magnet fields either the HMS or SOS spectrometers. The HMS has three focusing quadrupole magnets along with a bending dipole, while the SOS has a single focusing quadrupole magnet along with a pair of bending dipoles. The settings for each magnet are listed in Tbls. D.3 and D.4. 


\begin{tabular}{|c|c|c|c|c|}
\hline Central Momentum $(\mathrm{GeV})$ & $\mathrm{Q} 1(\mathrm{kG})$ & $\mathrm{Q} 2(\mathrm{kG})$ & $\mathrm{Q} 3(\mathrm{kG})$ & $\mathrm{D}(\mathrm{T})$ \\
\hline 4.7 & -8.3169 & 9.8168 & -4.7460 & 1.291838 \\
\hline 4.5 & -7.972 & 9.4106 & -4.540 & 1.23687 \\
\hline 3.9 & -6.9025 & 8.1547 & -3.9443 & 1.072035 \\
\hline 3.73 & -6.6088 & 7.8064 & -3.7668 & 1.0252213 \\
\hline 3.24 & -5.7346 & 6.7799 & -3.2798 & .890443 \\
\hline 3.1 & -5.4932 & 6.4904 & -3.132 & .8520647 \\
\hline 2.57 & -4.55441 & 5.3829 & -2.5981 & .7063789 \\
\hline 2.13 & -3.7722 & 4.4611 & -2.1575 & .585443 \\
\hline
\end{tabular}

Table D.3: HMS magnet settings.

\begin{tabular}{|c|c|c|c|}
\hline Central Momentum $(\mathrm{GeV})$ & $\mathrm{Q}(\mathrm{kG})$ & $\mathrm{D} 1(\mathrm{kG})$ & $\mathrm{D} 2(\mathrm{kG})$ \\
\hline 1.74 & -10.972 & -19.822 & 20.189 \\
\hline 1.04 & -6.576 & -11.853 & 12.070 \\
\hline
\end{tabular}

Table D.4: SOS magnet settings. 


\section{APPENDIX E Experimental Data}

\section{E.1 Differential Cross Sections}

The computed center of mass differential cross sections are displayed in this section. The statistical errors are always displayed on the inside error bar with a cross bar terminating their lengths. The sum (in quadrature) of the statistical and systematic errors are displayed on the outer error bars without, these do not have a cross bar. One can easily see that for the high SOS angle setting (high $Q^{2}$ ) the acceptance falls off at the higher values of invariant energy $W$. The curves fit through the data correspond to the functional form described in Section 10.3. 


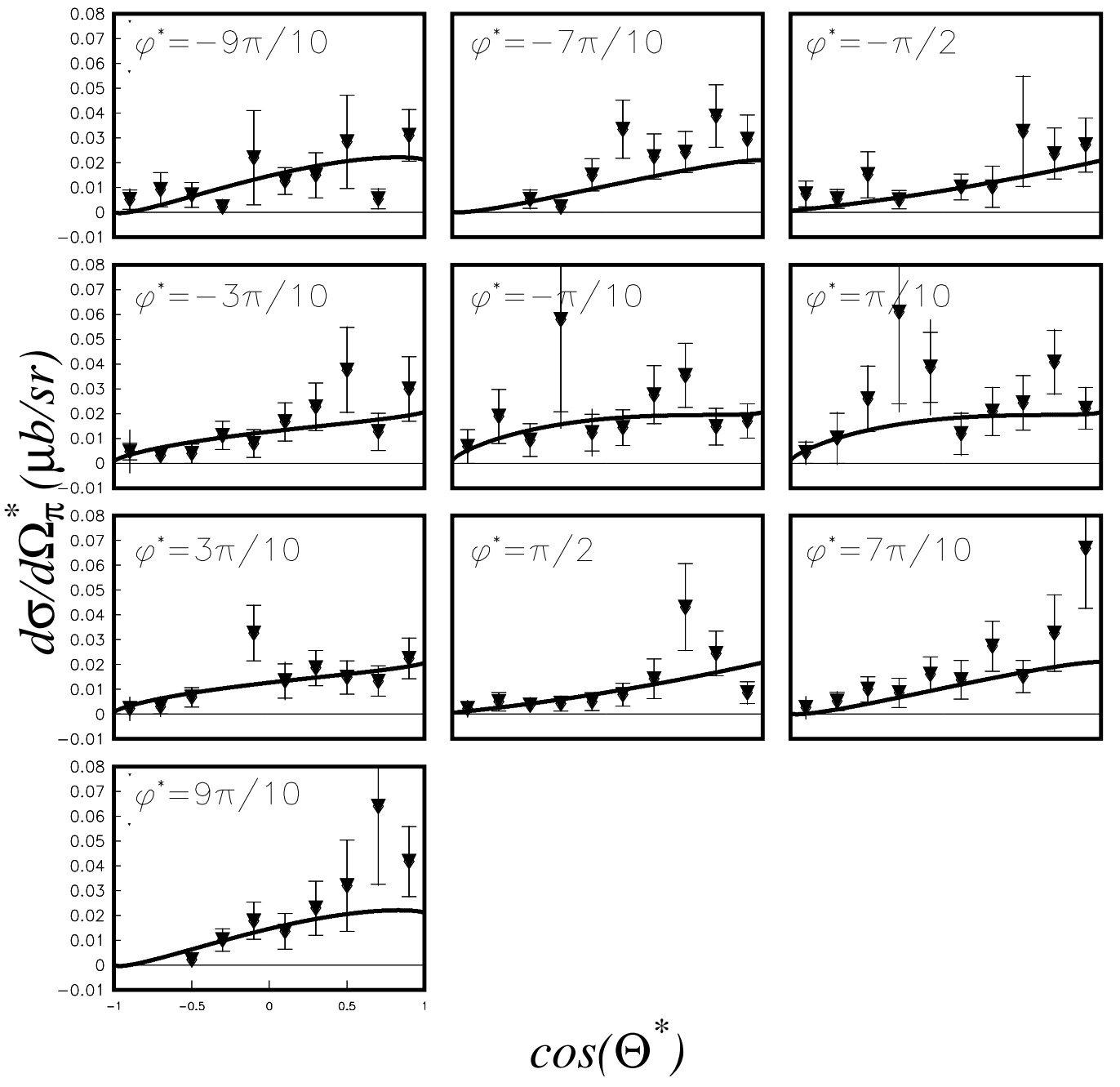

Figure E.1: Center of mass differential cross sections for $W=1.112 \mathrm{GeV}$ and $Q^{2}=6.564 \mathrm{GeV}^{2} \cdot \frac{\chi^{2}}{n_{\text {dof }}}=1.145$. 


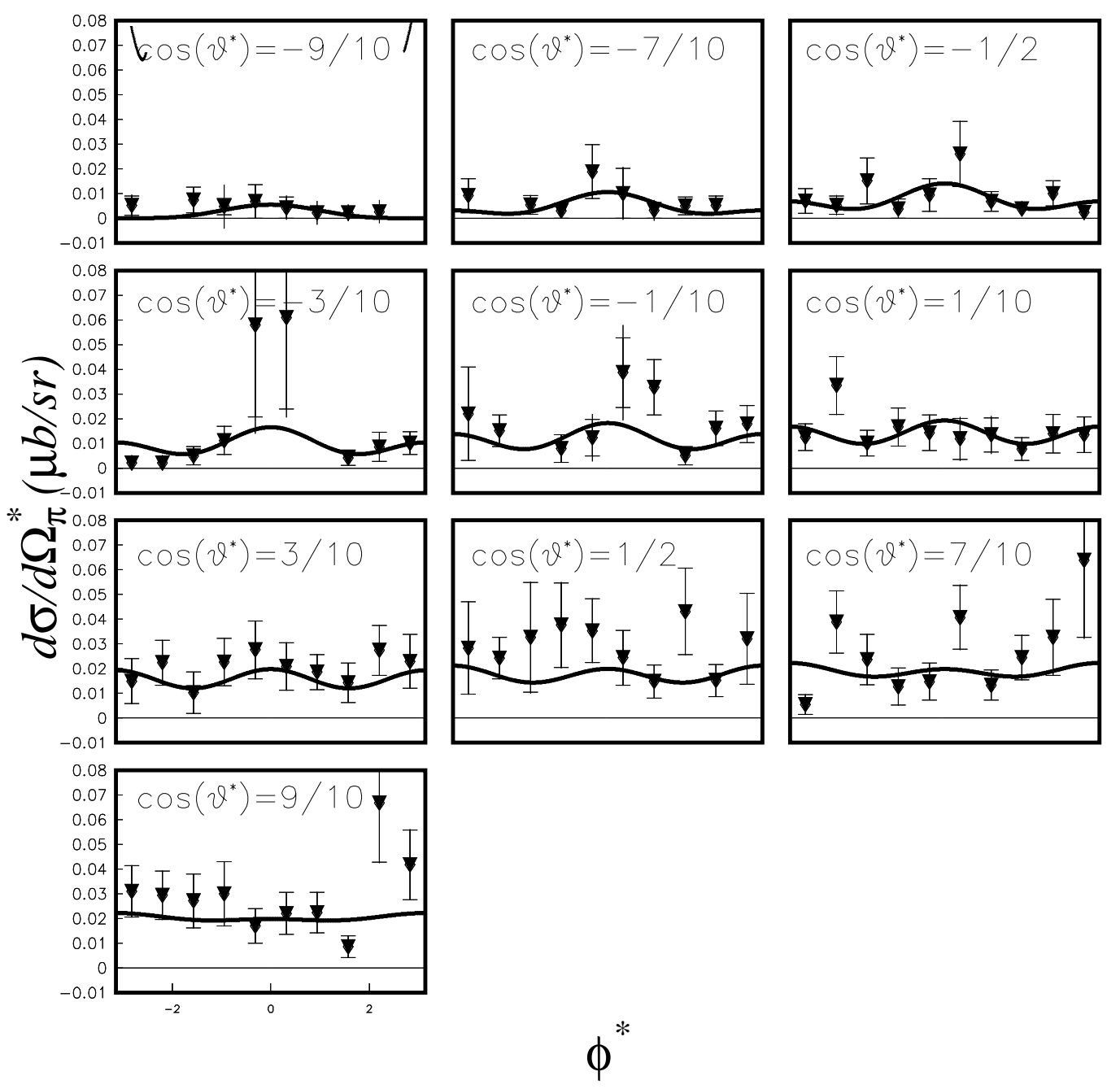

Figure E.2: Center of mass differential cross sections for $W=1.112 \mathrm{GeV}$ and $Q^{2}=6.564 \mathrm{GeV}^{2} \cdot \frac{\chi^{2}}{n_{d o f}}=1.145$. 


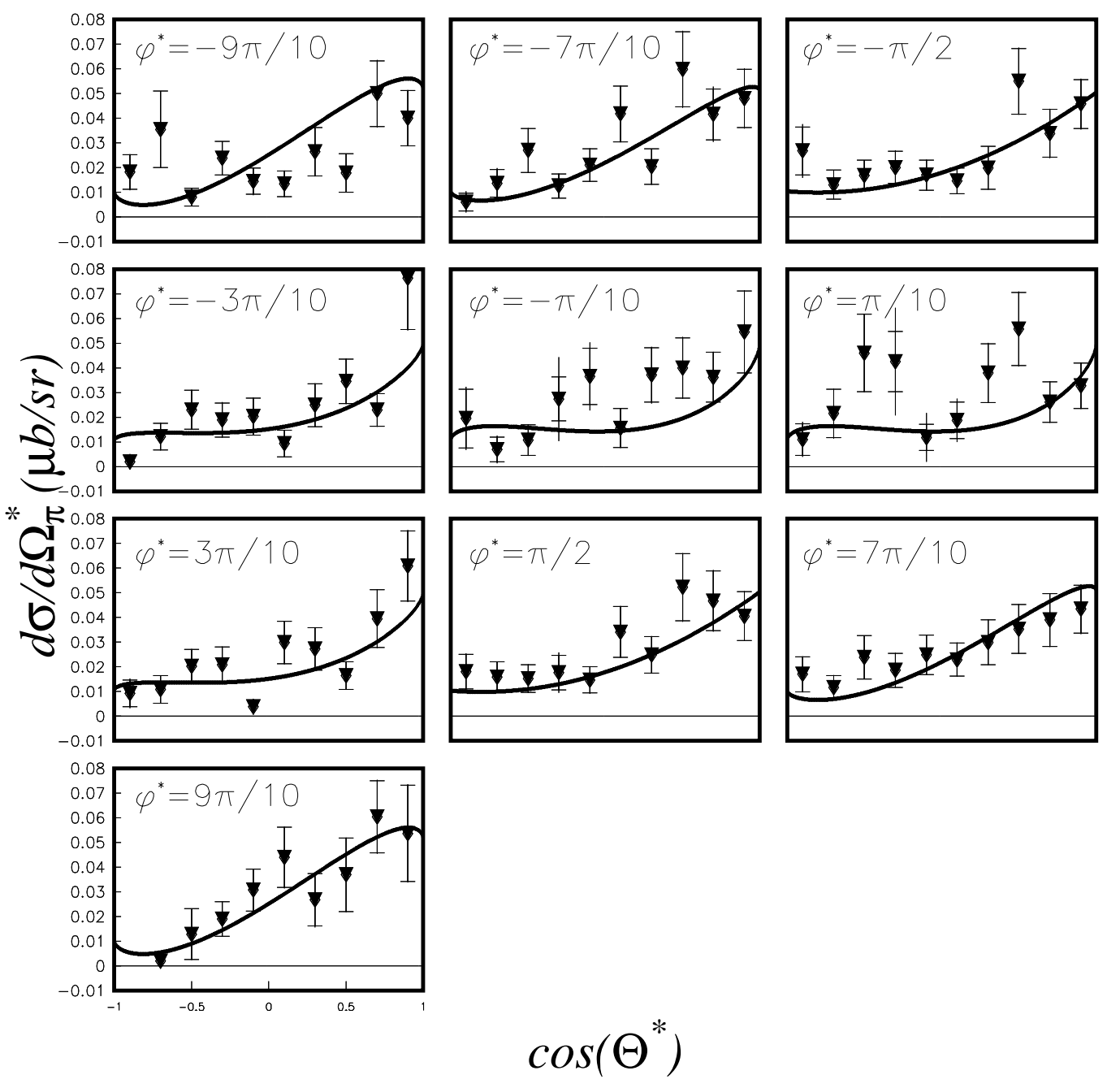

Figure E.3: Center of mass differential cross sections for $W=1.152 \mathrm{GeV}$ and $Q^{2}=6.5 \mathrm{GeV}^{2} \cdot \frac{\chi^{2}}{n_{d o f}}=1.082$. 


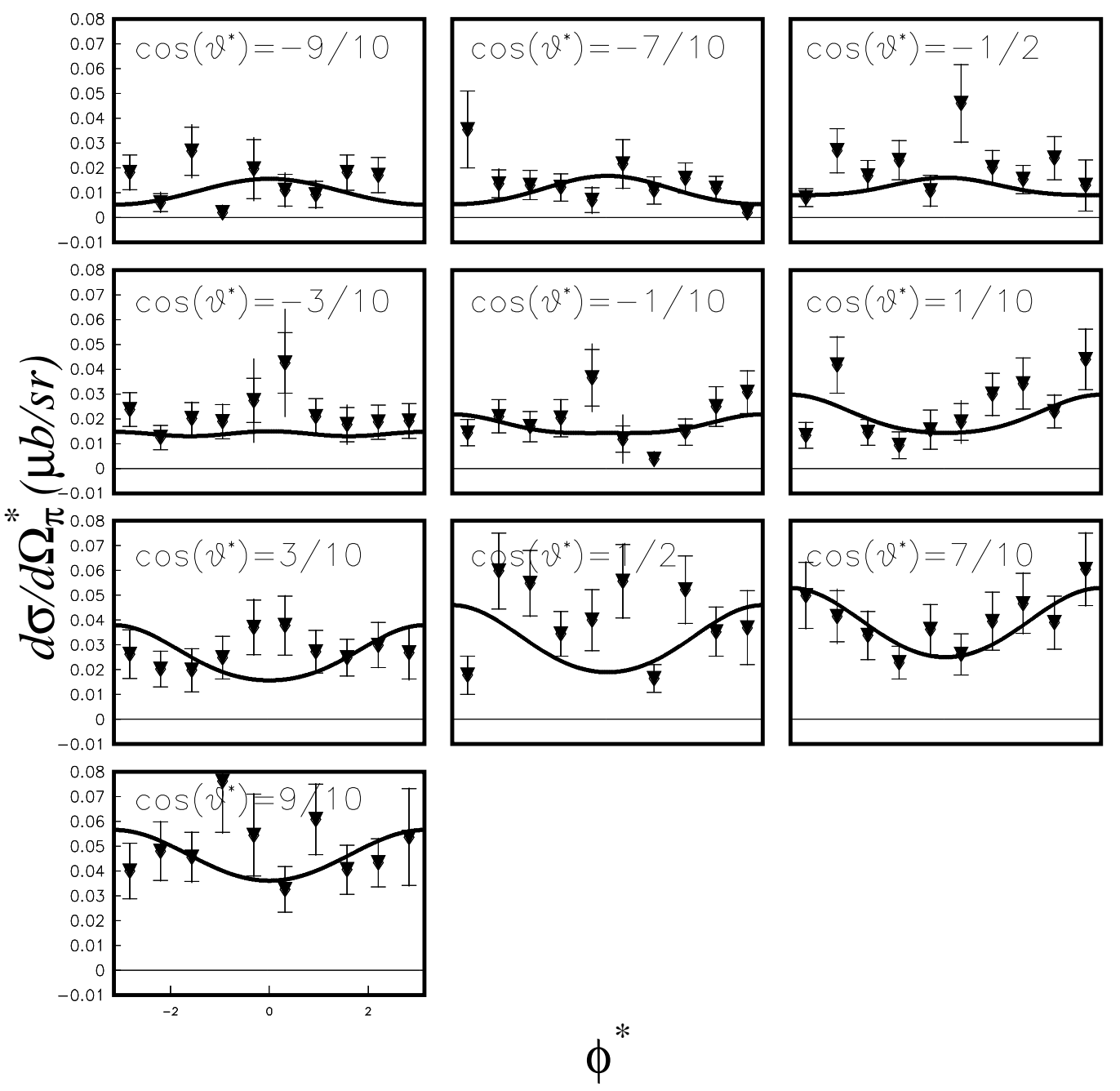

Figure E.4: Center of mass differential cross sections for $W=1.152 \mathrm{GeV}$ and $Q^{2}=6.5 \mathbf{G e V}^{2} \cdot \frac{\chi^{2}}{n_{d o f}}=1.082$. 

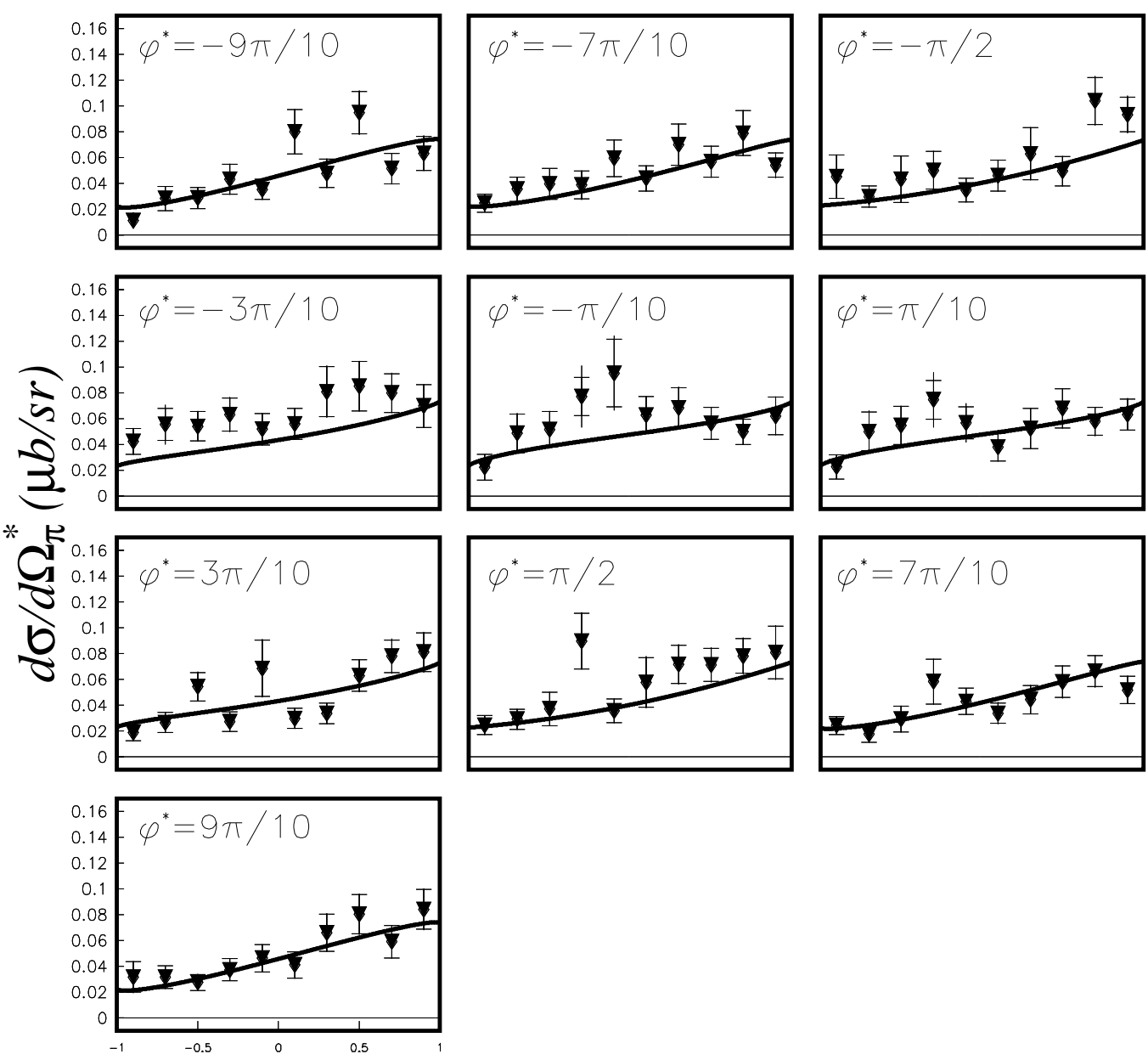

$$
\cos \left(\Theta^{*}\right)
$$

Figure E.5: Center of mass differential cross sections for $W=1.192 \mathrm{GeV}$ and $Q^{2}=6.432 \mathrm{GeV}^{2} \cdot \frac{\chi^{2}}{n_{\text {dof }}}=1.279$. 

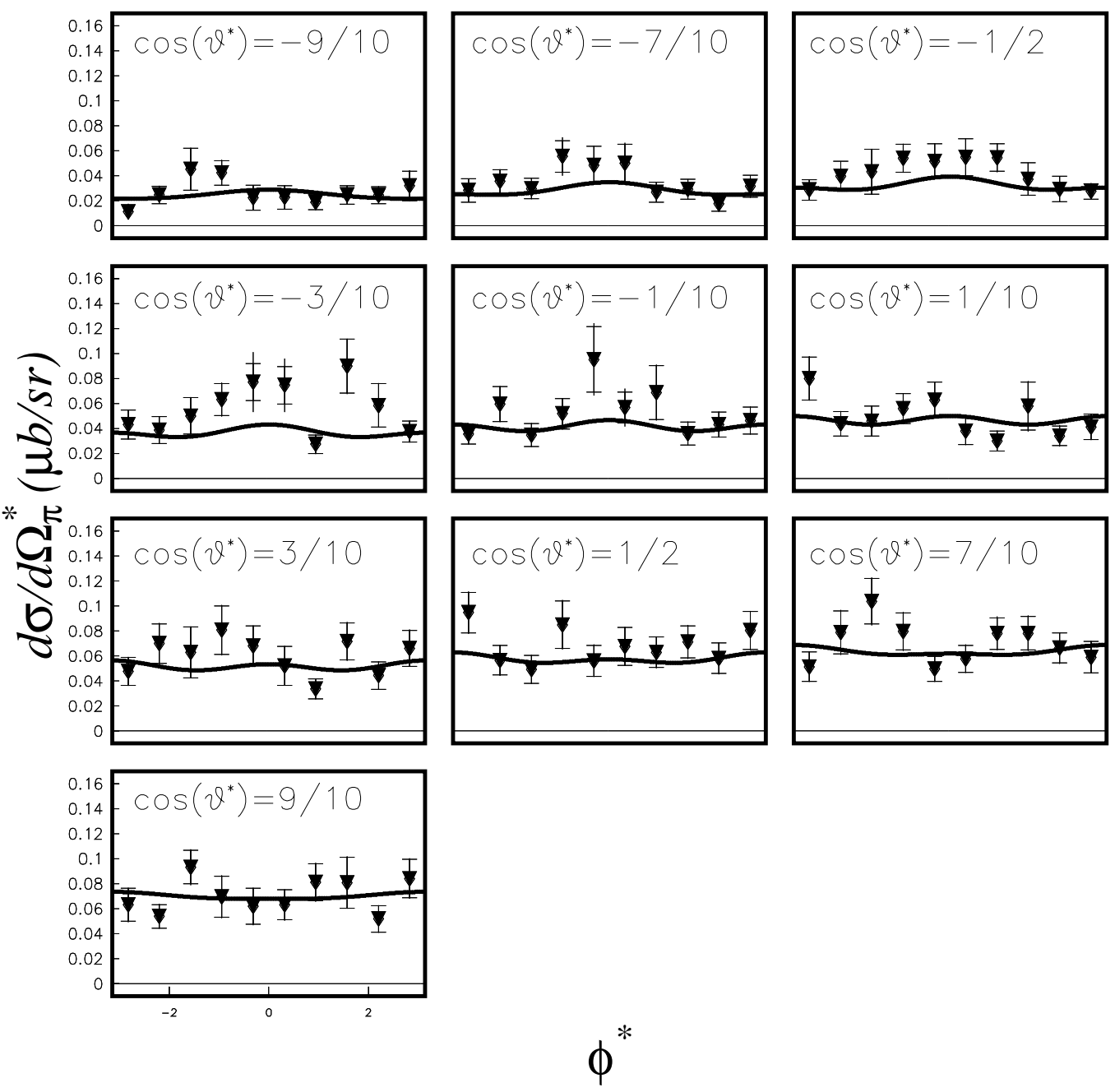

Figure E.6: Center of mass differential cross sections for $W=1.192 \mathrm{GeV}$ and $Q^{2}=6.432 \mathrm{GeV}^{2} \cdot \frac{\chi^{2}}{n_{\text {dof }}}=1.279$. 

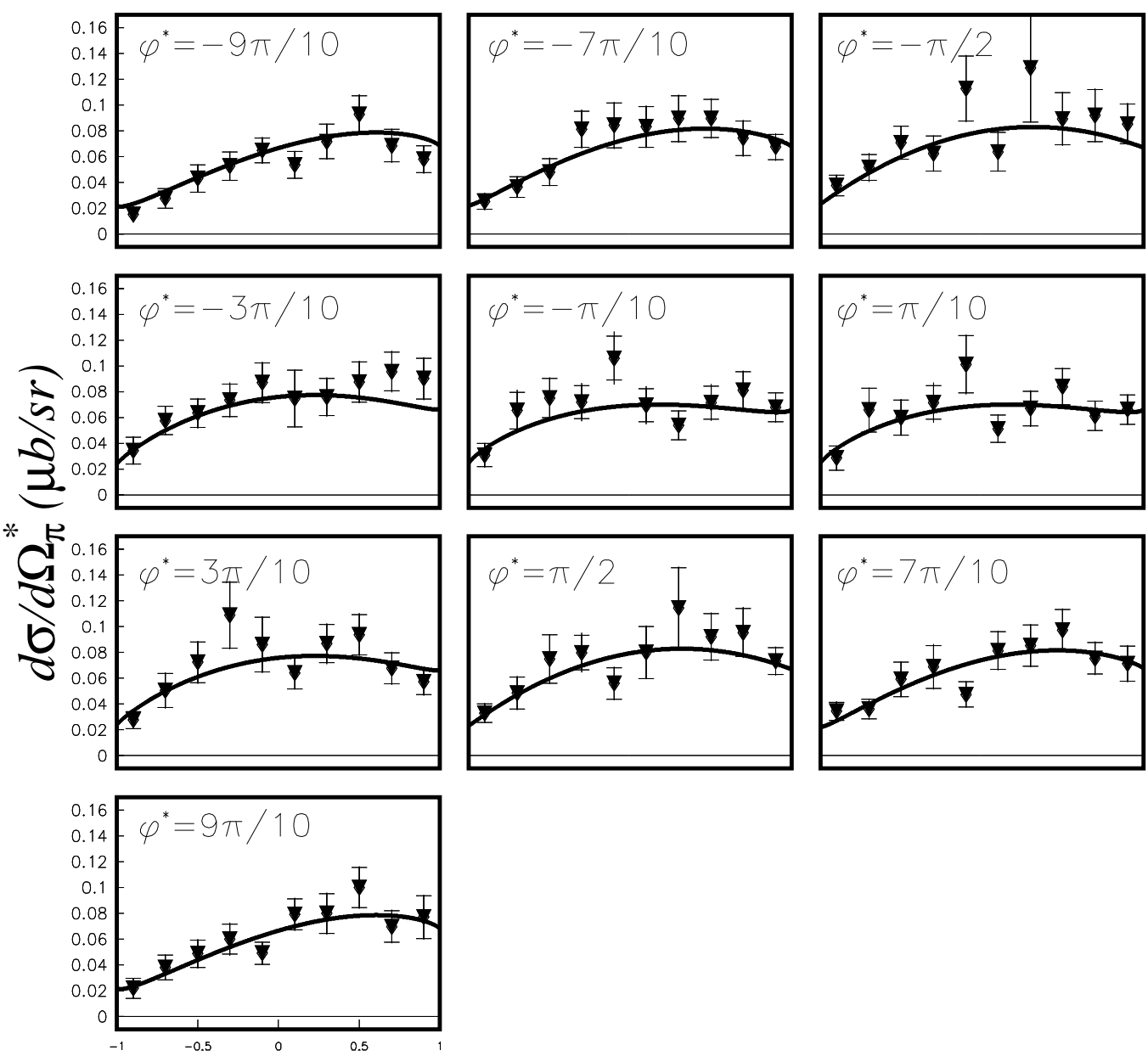

$$
\cos \left(\Theta^{*}\right)
$$

Figure E.7: Center of mass differential cross sections for $W=1.232 \mathrm{GeV}$ and $Q^{2}=6.36 \mathrm{GeV}^{2} \cdot \frac{\chi^{2}}{n_{d o f}}=0.861$. 


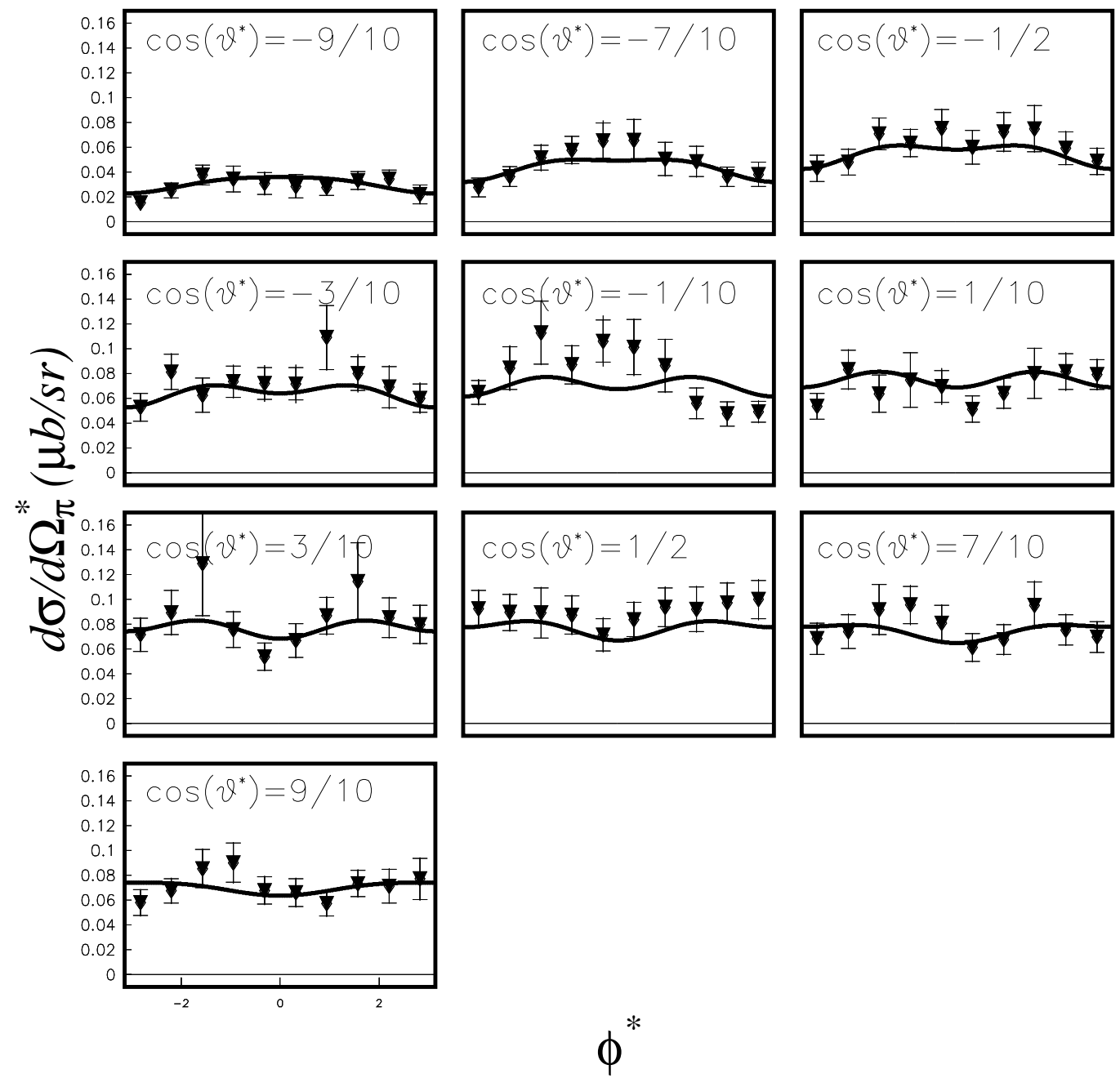

Figure E.8: Center of mass differential cross sections for $W=1.232 \mathrm{GeV}$ and $Q^{2}=6.36 \mathrm{GeV}^{2} \cdot \frac{\chi^{2}}{n_{\text {dof }}}=0.861$. 


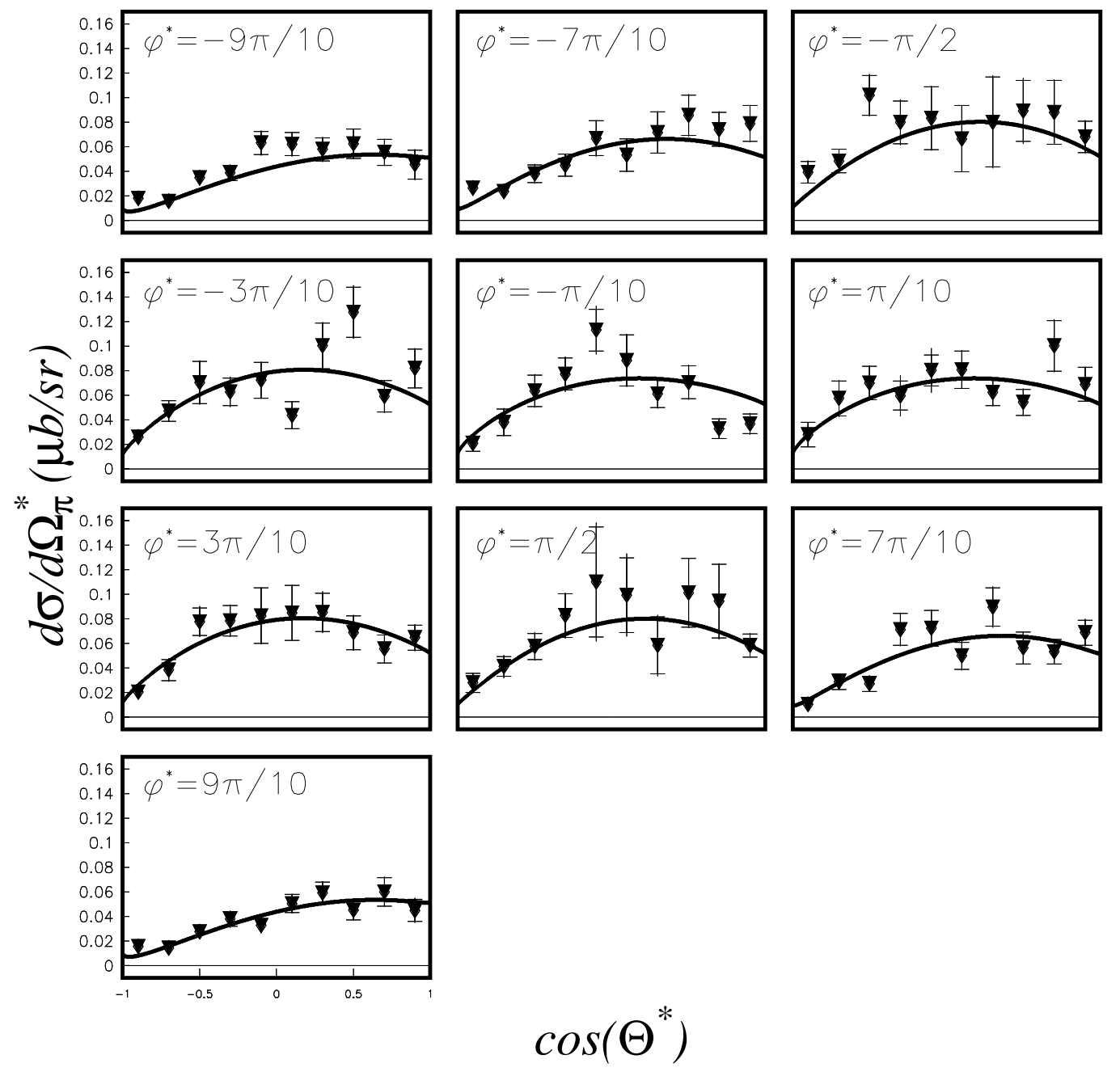

Figure E.9: Center of mass differential cross sections for $W=1.272 \mathrm{GeV}$ and $Q^{2}=6.288 \mathrm{GeV}^{2} \cdot \frac{\chi^{2}}{n_{\text {dof }}}=1.795$. 

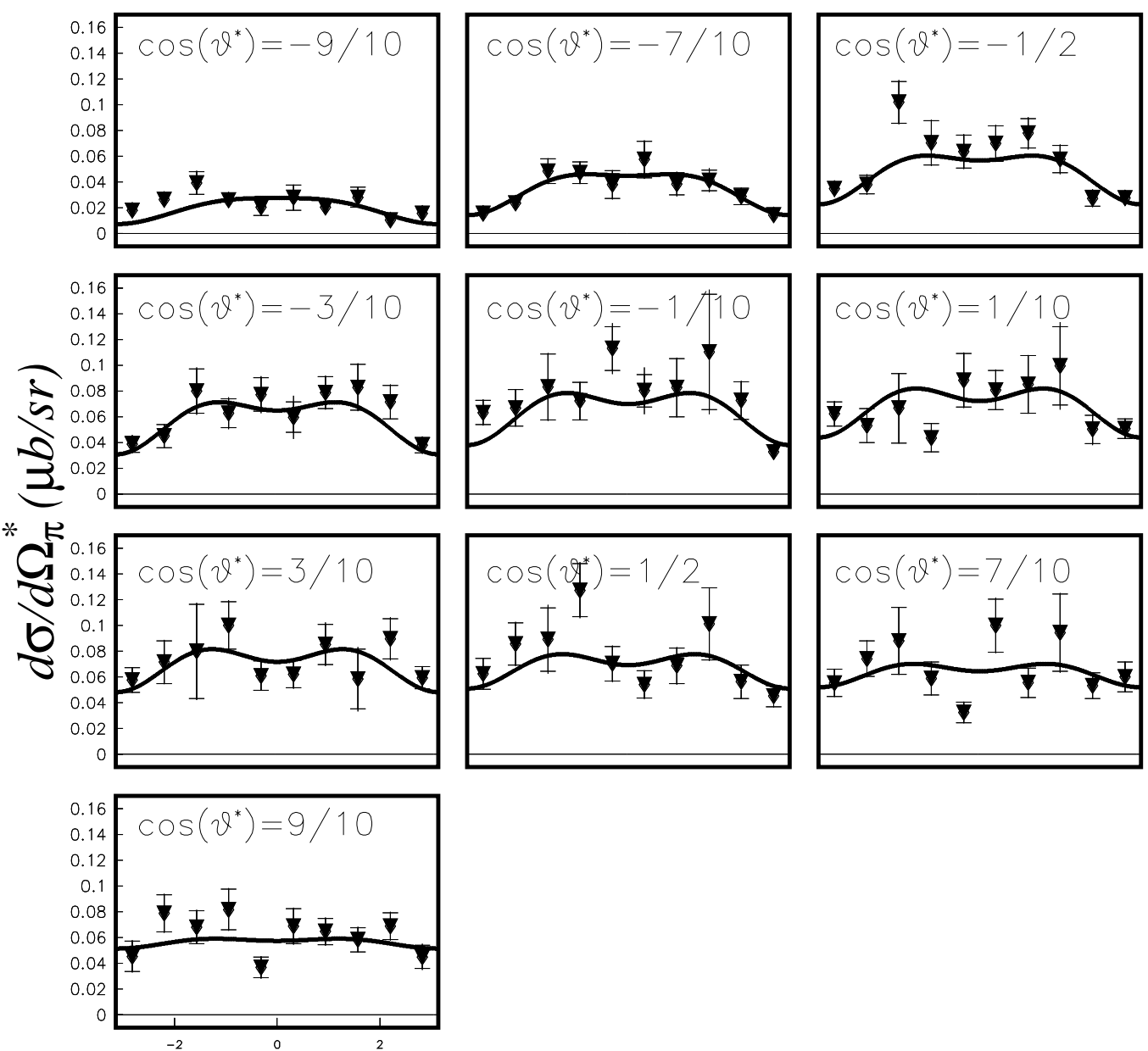

$\phi^{*}$

Figure E.10: Center of mass differential cross sections for $W=1.272 \mathrm{GeV}$ and $Q^{2}=6.288 \mathrm{GeV}^{2} \cdot \frac{\chi^{2}}{n_{\text {dof }}}=1.795$. 


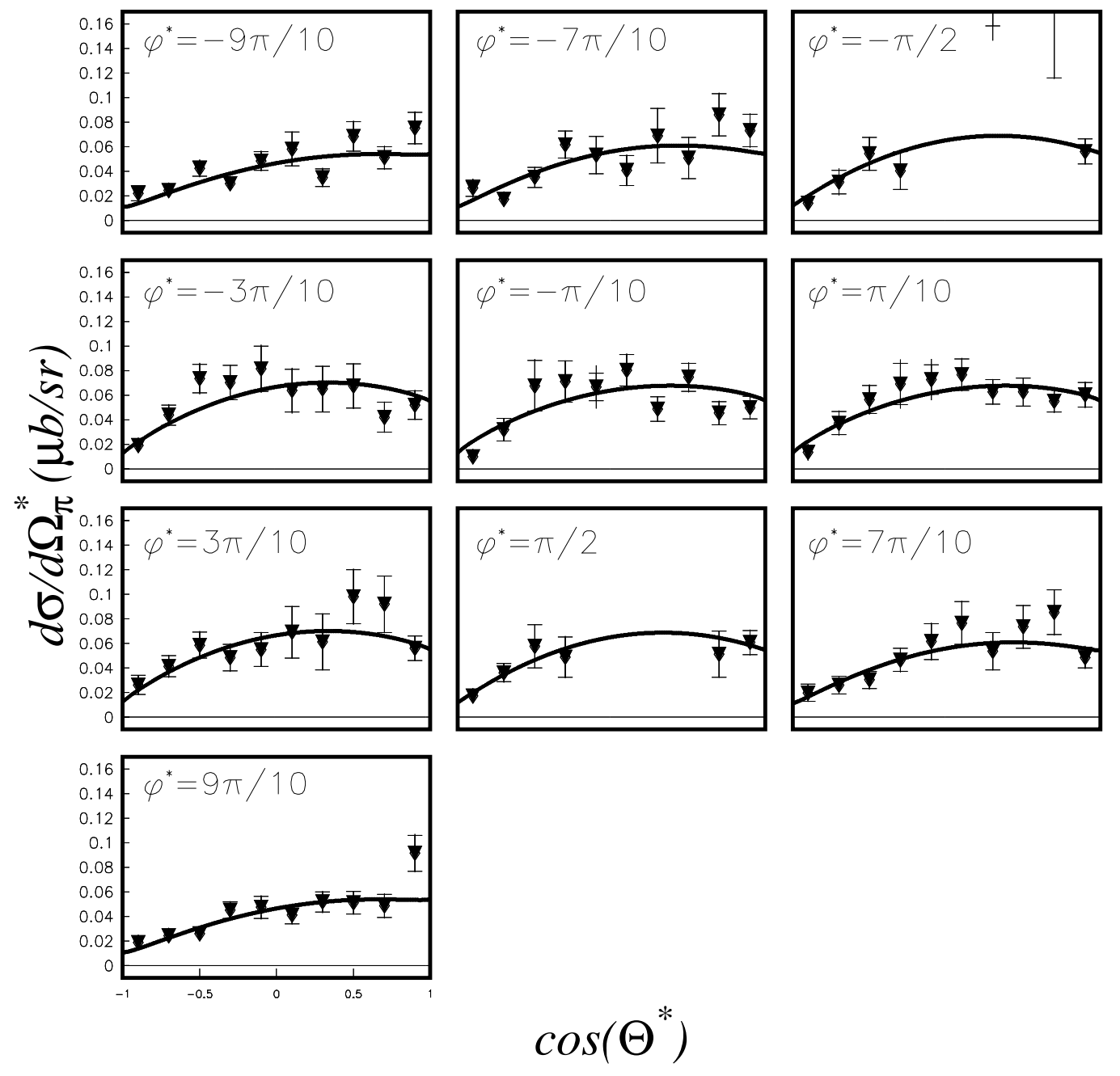

Figure E.11: Center of mass differential cross sections for $W=1.312 \mathrm{GeV}$ and $Q^{2}=6.212 \mathrm{GeV}^{2} \cdot \frac{\chi^{2}}{n_{\text {dof }}}=1.595$. 

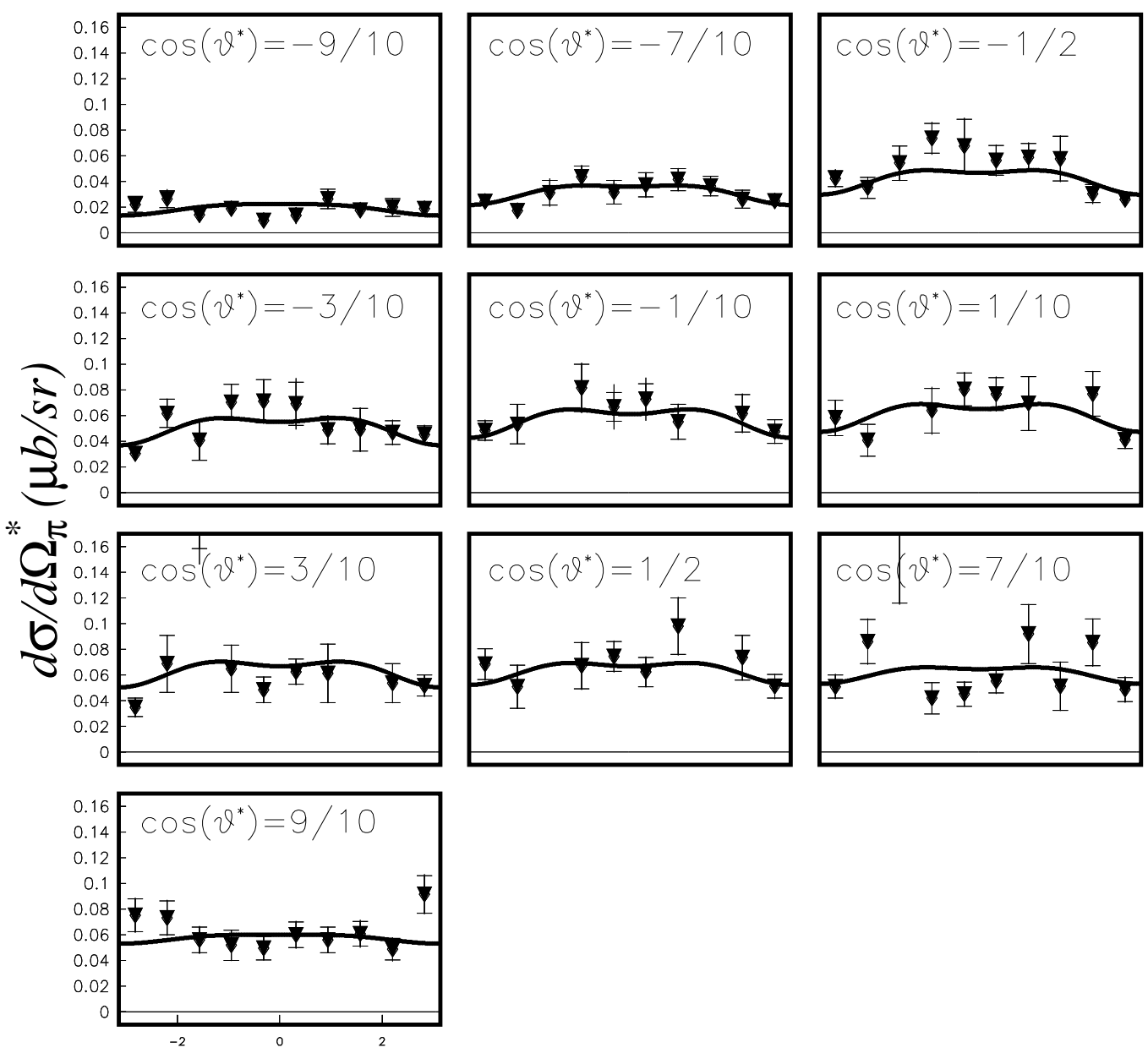

$\phi^{*}$

Figure E.12: Center of mass differential cross sections for $W=1.312 \mathrm{GeV}$ and $Q^{2}=6.212 \mathrm{GeV}^{2} \cdot \frac{\chi^{2}}{n_{\text {dof }}}=1.595$. 

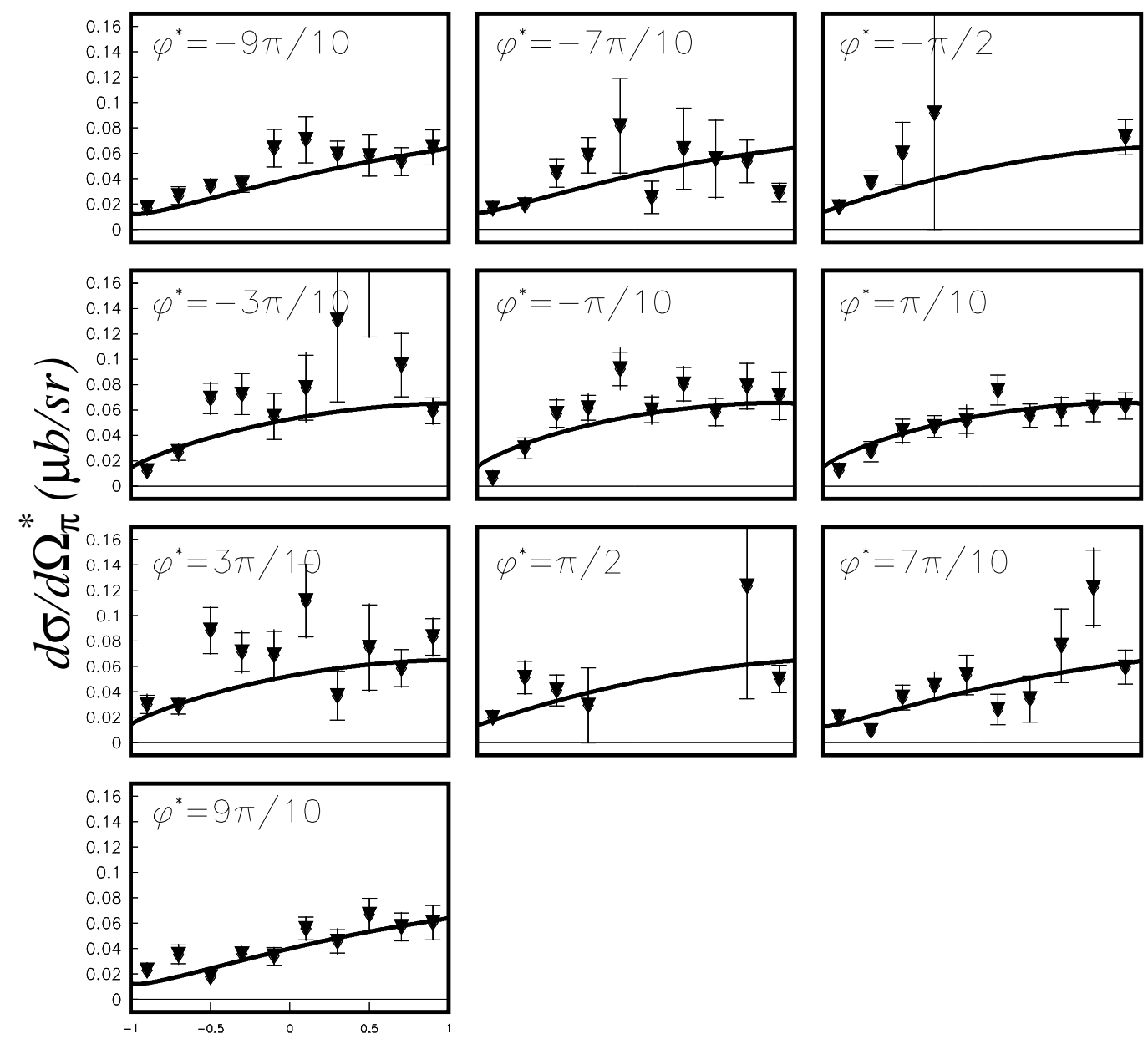

$$
\cos \left(\Theta^{*}\right)
$$

Figure E.13: Center of mass differential cross sections for $W=1.352 \mathrm{GeV}$ and $Q^{2}=6.136 \mathrm{GeV}^{2} \cdot \frac{\chi^{2}}{n_{\text {dof }}}=2.185$. 


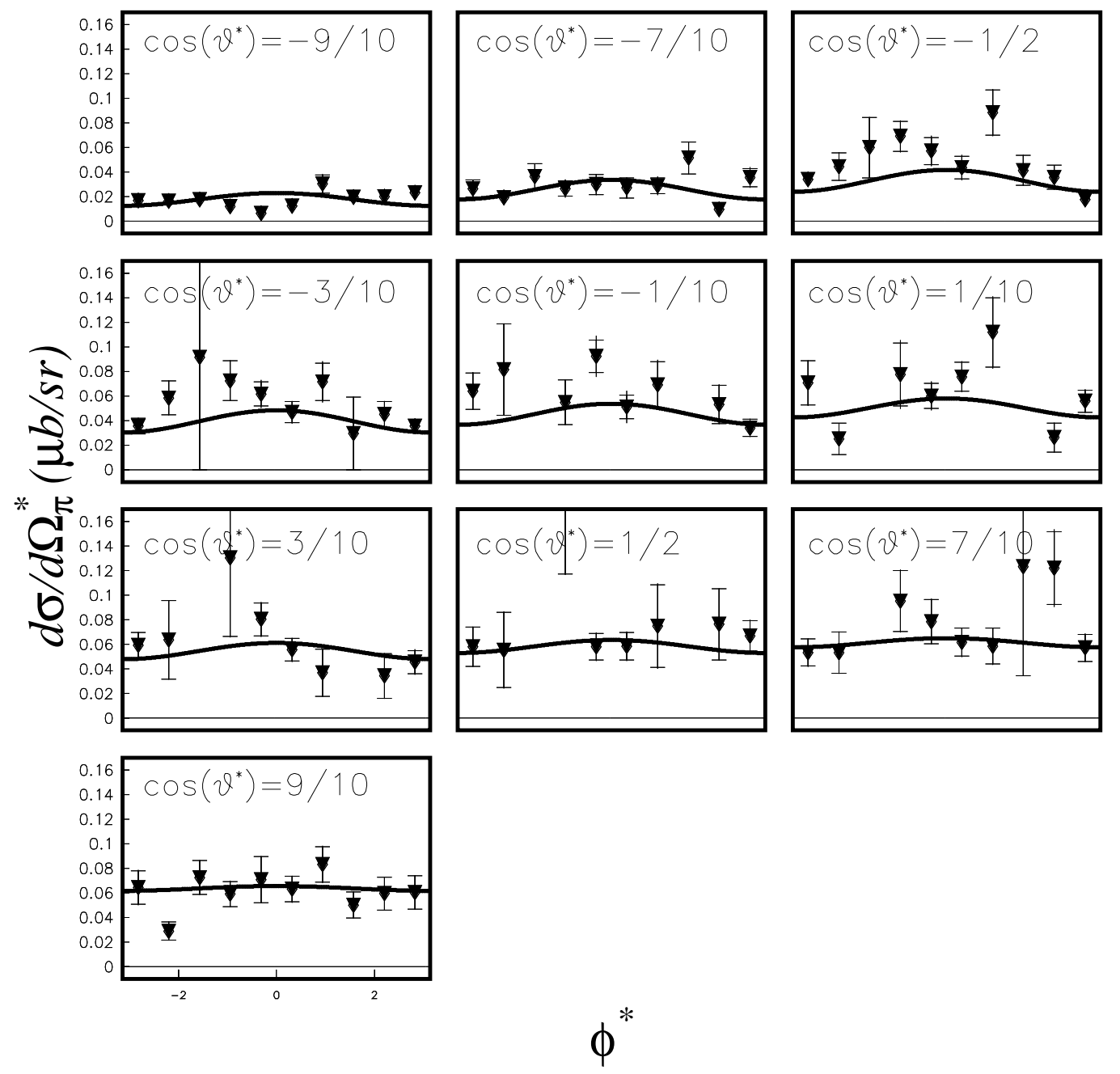

Figure E.14: Center of mass differential cross sections for $W=1.352 \mathrm{GeV}$ and $Q^{2}=6.136 \mathrm{GeV}^{2} \cdot \frac{\chi^{2}}{n_{\text {dof }}}=2.185$. 


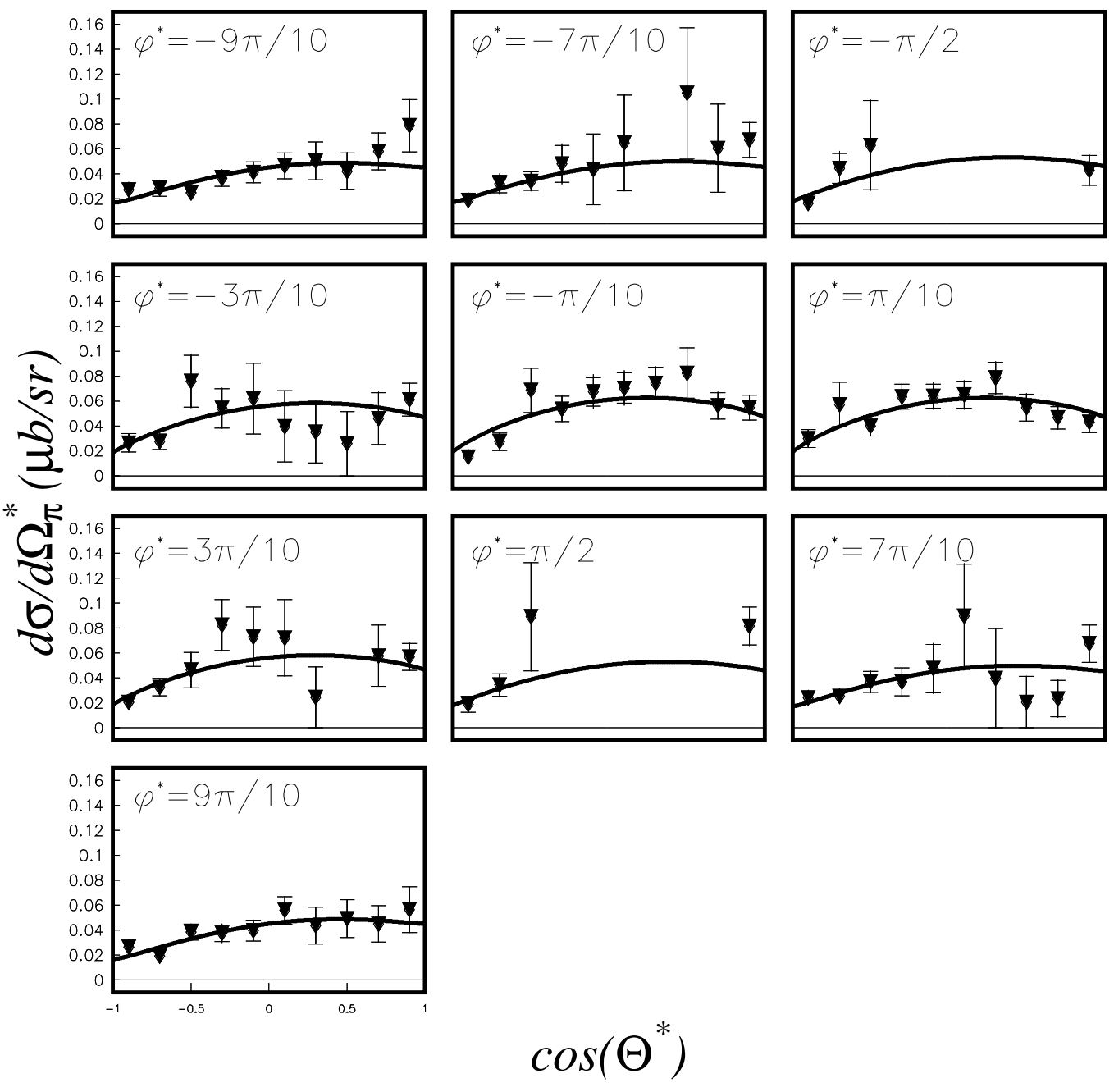

Figure E.15: Center of mass differential cross sections for $W=1.392 \mathrm{GeV}$ and $Q^{2}=6.06 \mathrm{GeV}^{2} \cdot \frac{\chi^{2}}{n_{\text {dof }}}=1.249$. 

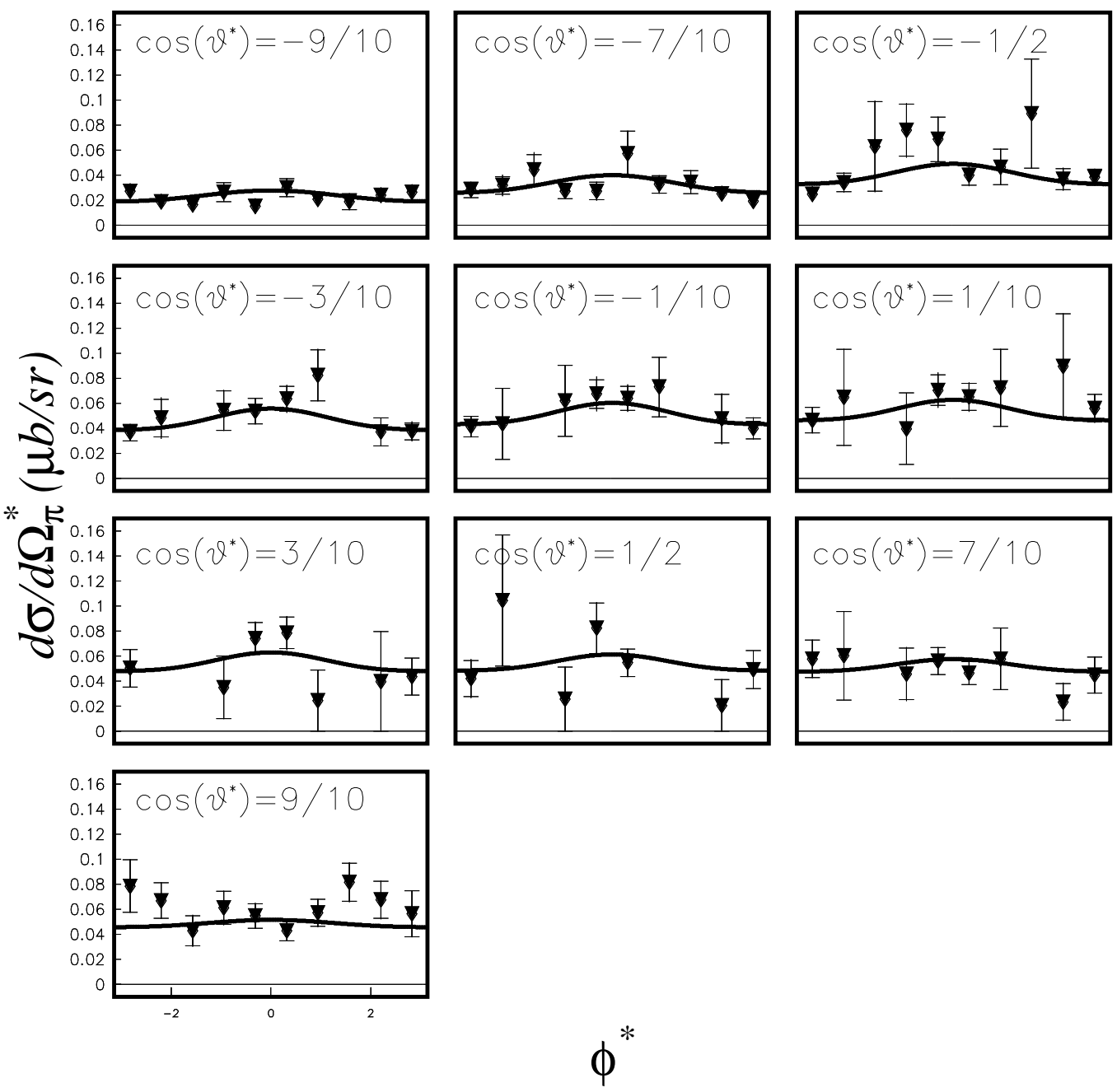

Figure E.16: Center of mass differential cross sections for $W=1.392 \mathrm{GeV}$ and $Q^{2}=6.06 \mathrm{GeV}^{2} \cdot \frac{\chi^{2}}{n_{d o f}}=1.249$. 


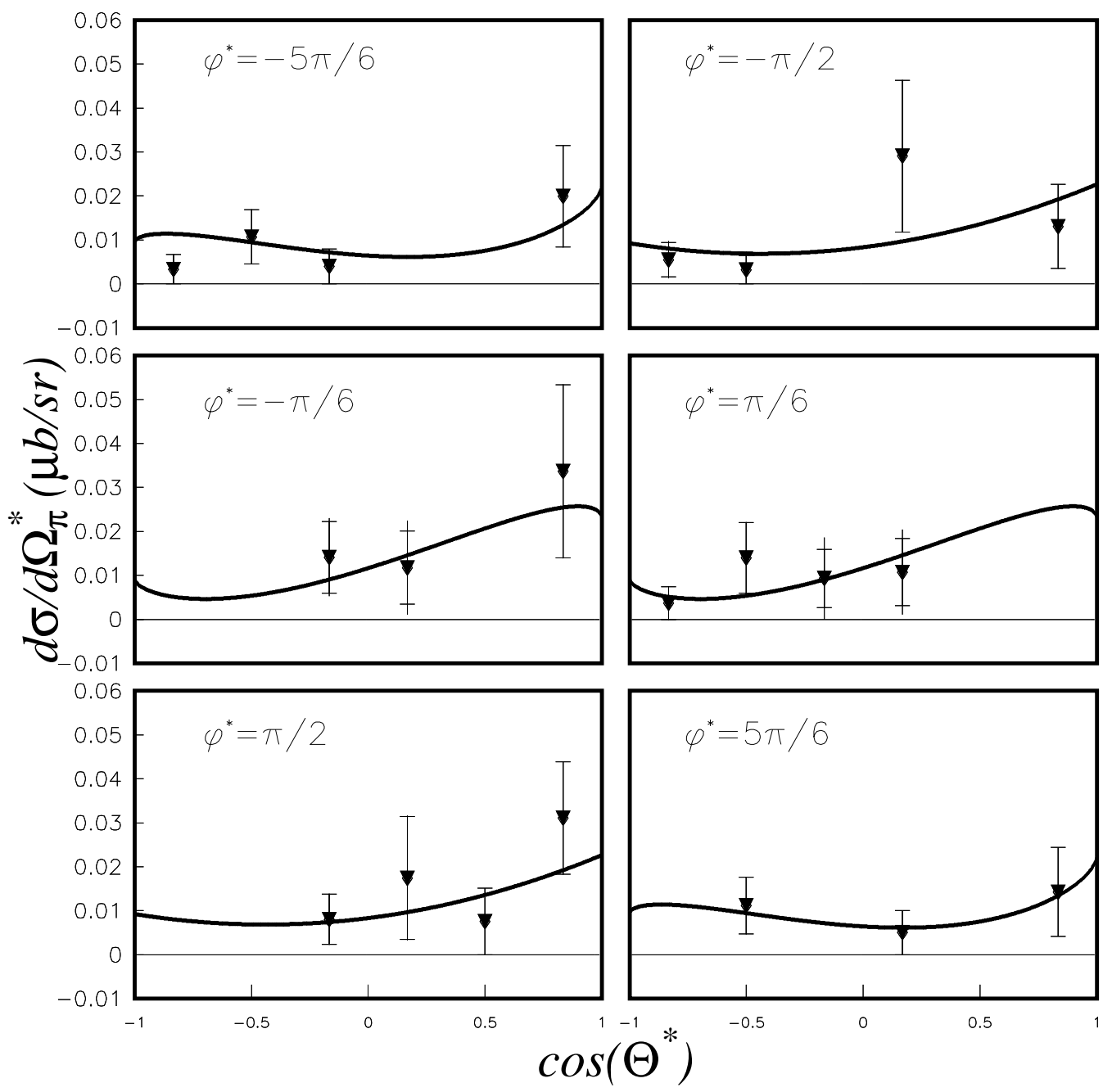

Figure E.17: Center of mass differential cross sections for $W=1.112 \mathrm{GeV}$ and $Q^{2}=7.924 \mathrm{GeV}^{2} \cdot \frac{\chi^{2}}{n_{\text {dof }}}=0.645$.

The fits for the higher $Q^{2}$ data are underconstrained because of lack of statistics. These data are still good but the fits are not trustable. 


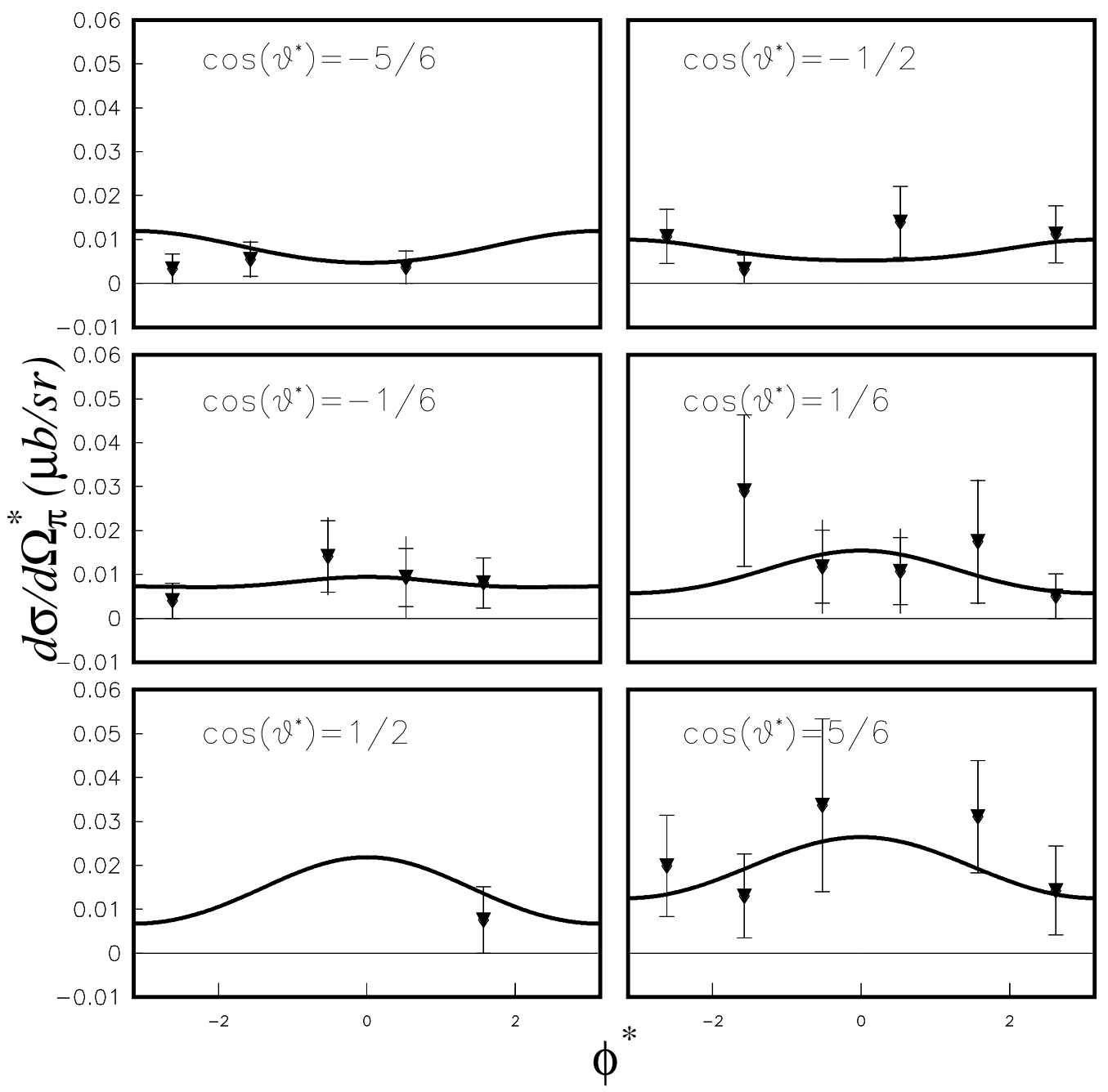

Figure E.18: Center of mass differential cross sections for $W=1.112 \mathrm{GeV}$ and $Q^{2}=7.924 \mathrm{GeV}^{2} \cdot \frac{\chi^{2}}{n_{\text {dof }}}=0.645$. 


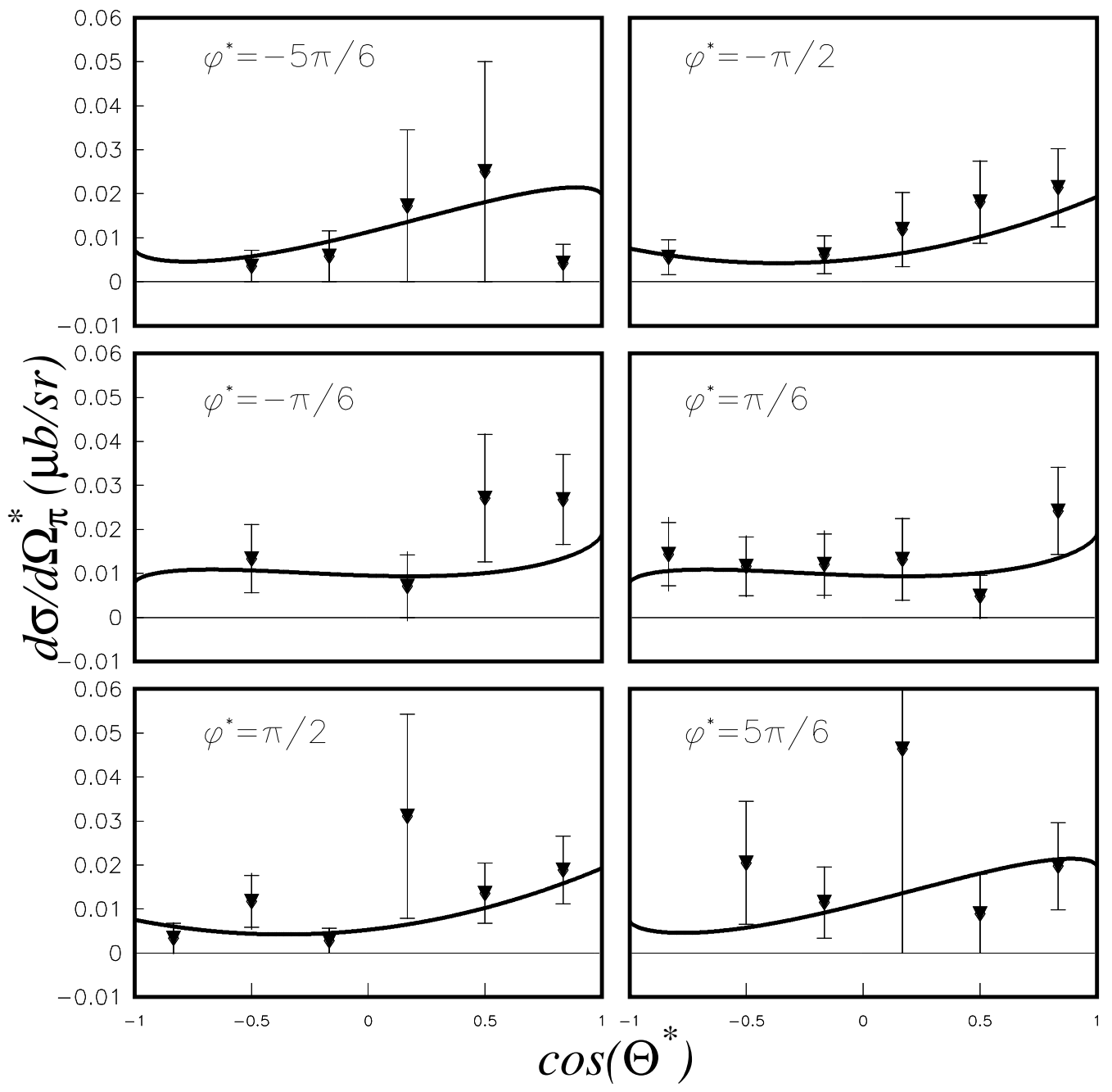

Figure E.19: Center of mass differential cross sections for $W=1.152 \mathrm{GeV}$ and $Q^{2}=7.848 \mathrm{GeV}^{2} \cdot \frac{\chi^{2}}{n_{\text {dof }}}=0.642$. 


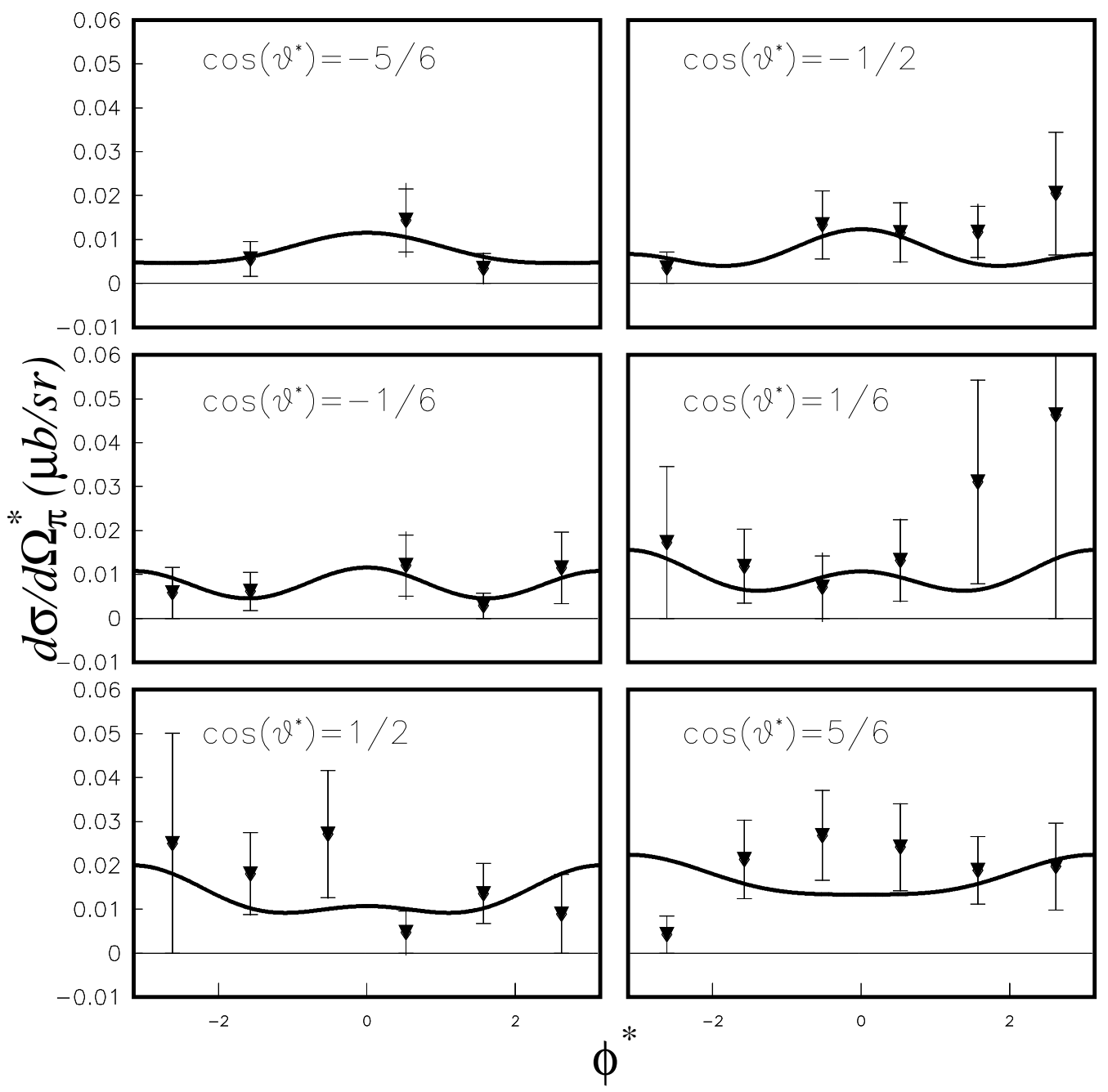

Figure E.20: Center of mass differential cross sections for $W=1.152 \mathrm{GeV}$ and $Q^{2}=7.848 \mathrm{GeV}^{2} \cdot \frac{\chi^{2}}{n_{\text {dof }}}=0.642$. 

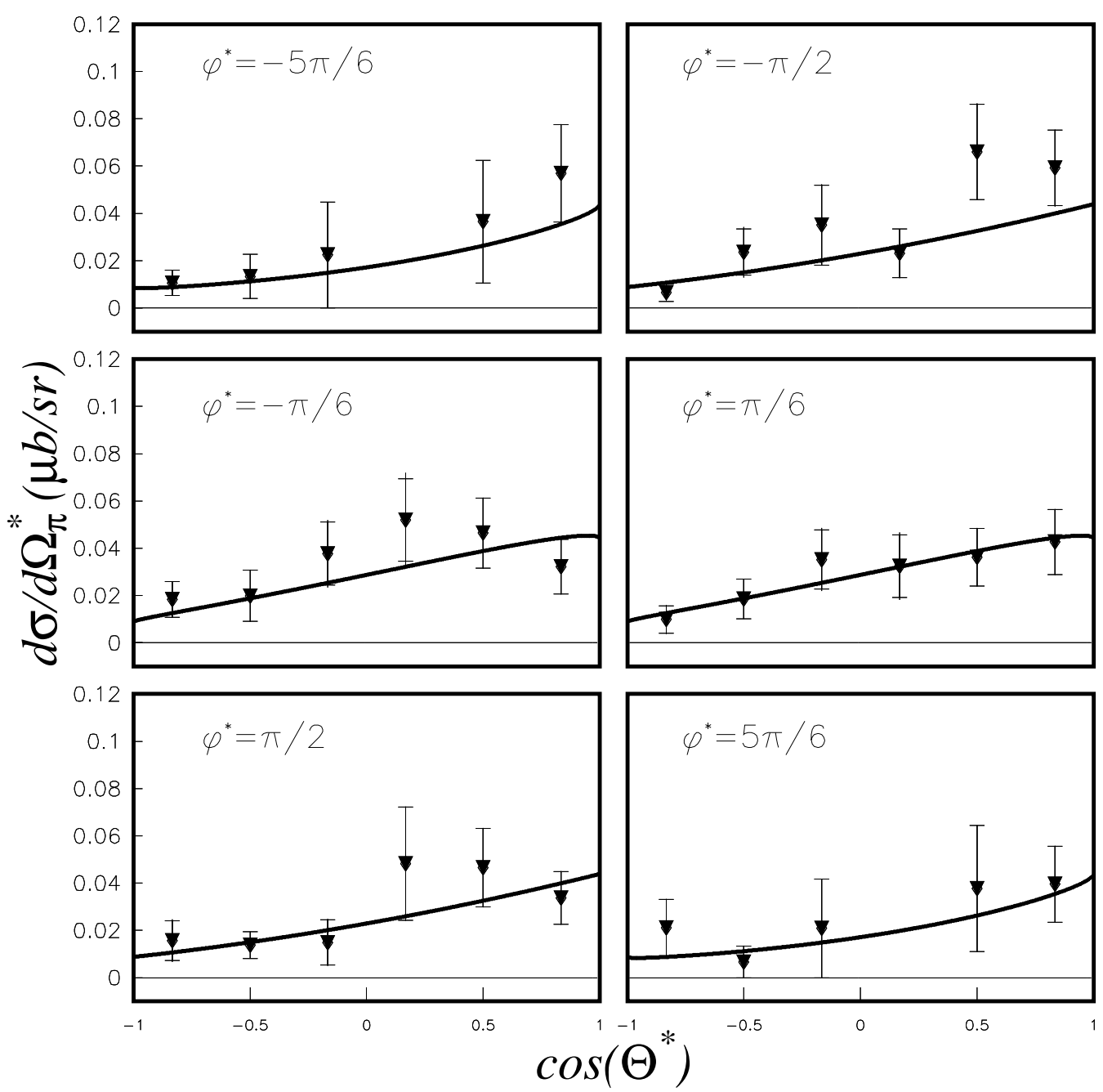

Figure E.21: Center of mass differential cross sections for $W=1.192 \mathrm{GeV}$ and $Q^{2}=7.772 \mathrm{GeV}^{2} \cdot \frac{\chi^{2}}{n_{\text {dof }}}=0.583$. 

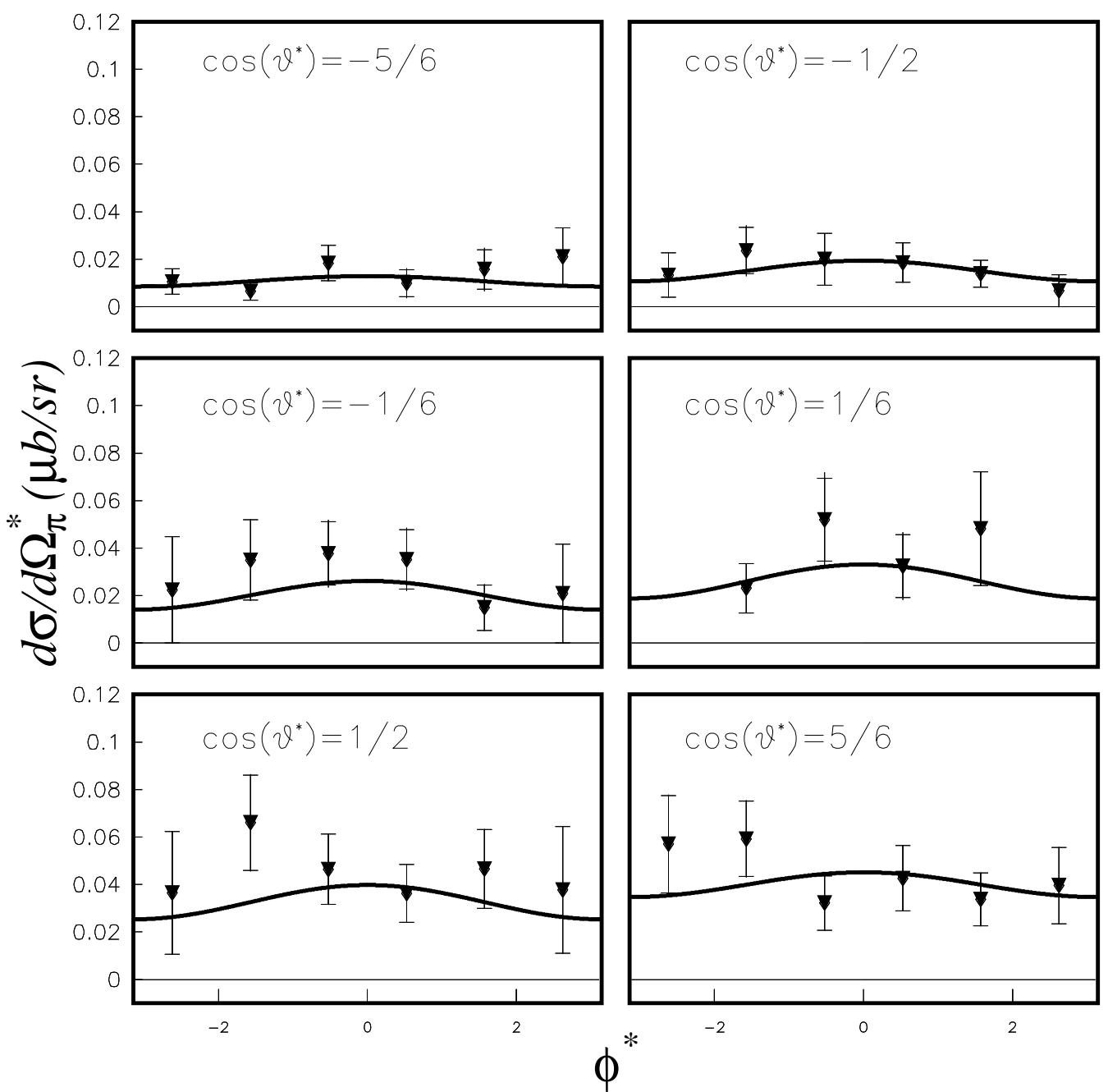

Figure E.22: Center of mass differential cross sections for $W=1.192 \mathrm{GeV}$ and $Q^{2}=7.772 \mathrm{GeV}^{2} \cdot \frac{\chi^{2}}{n_{\text {dof }}}=0.583$. 

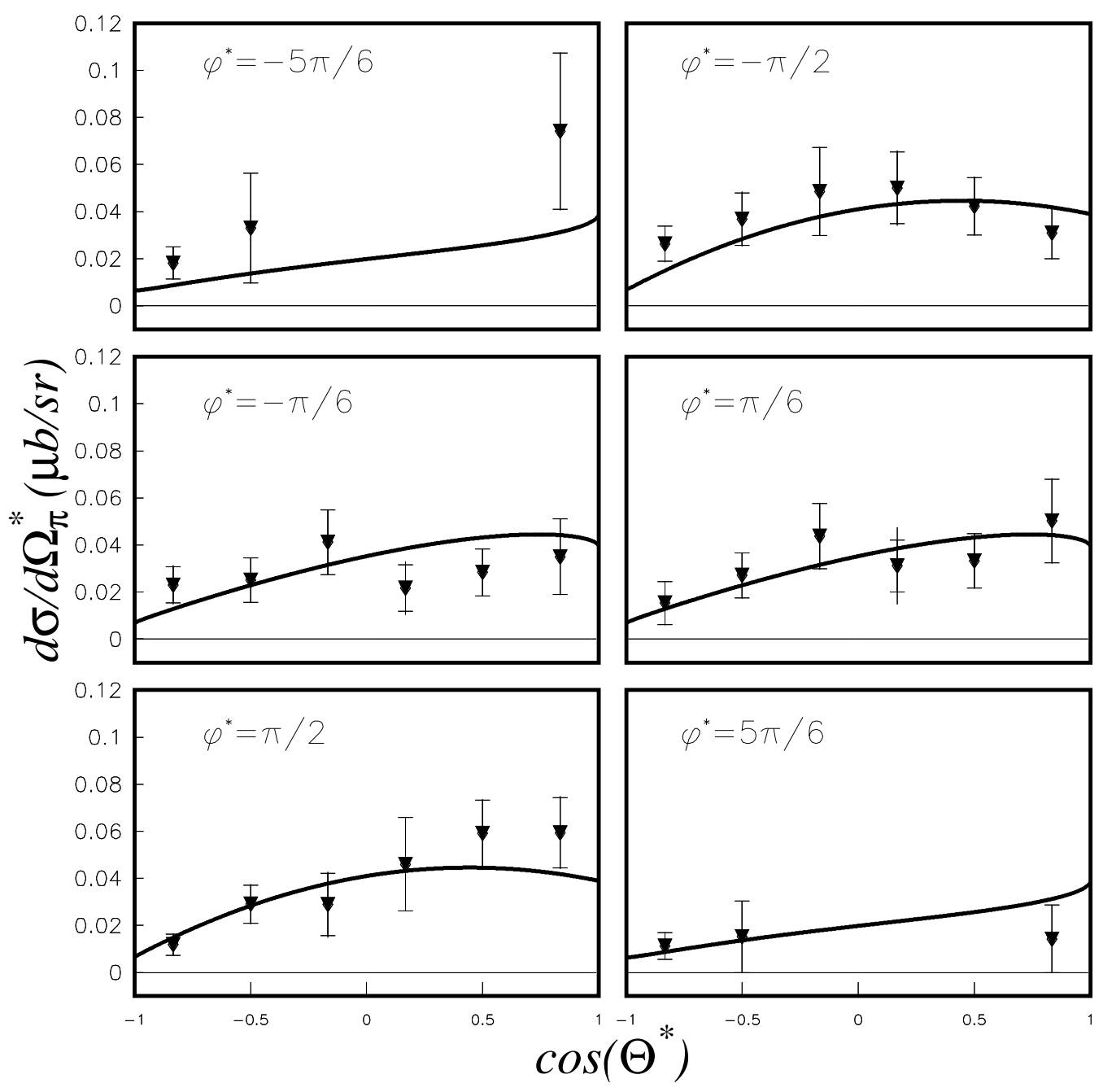

Figure E.23: Center of mass differential cross sections for $W=1.232 \mathrm{GeV}$ and $Q^{2}=7.692 \mathrm{GeV}^{2} \cdot \frac{\chi^{2}}{n_{\text {dof }}}=0.785$. 

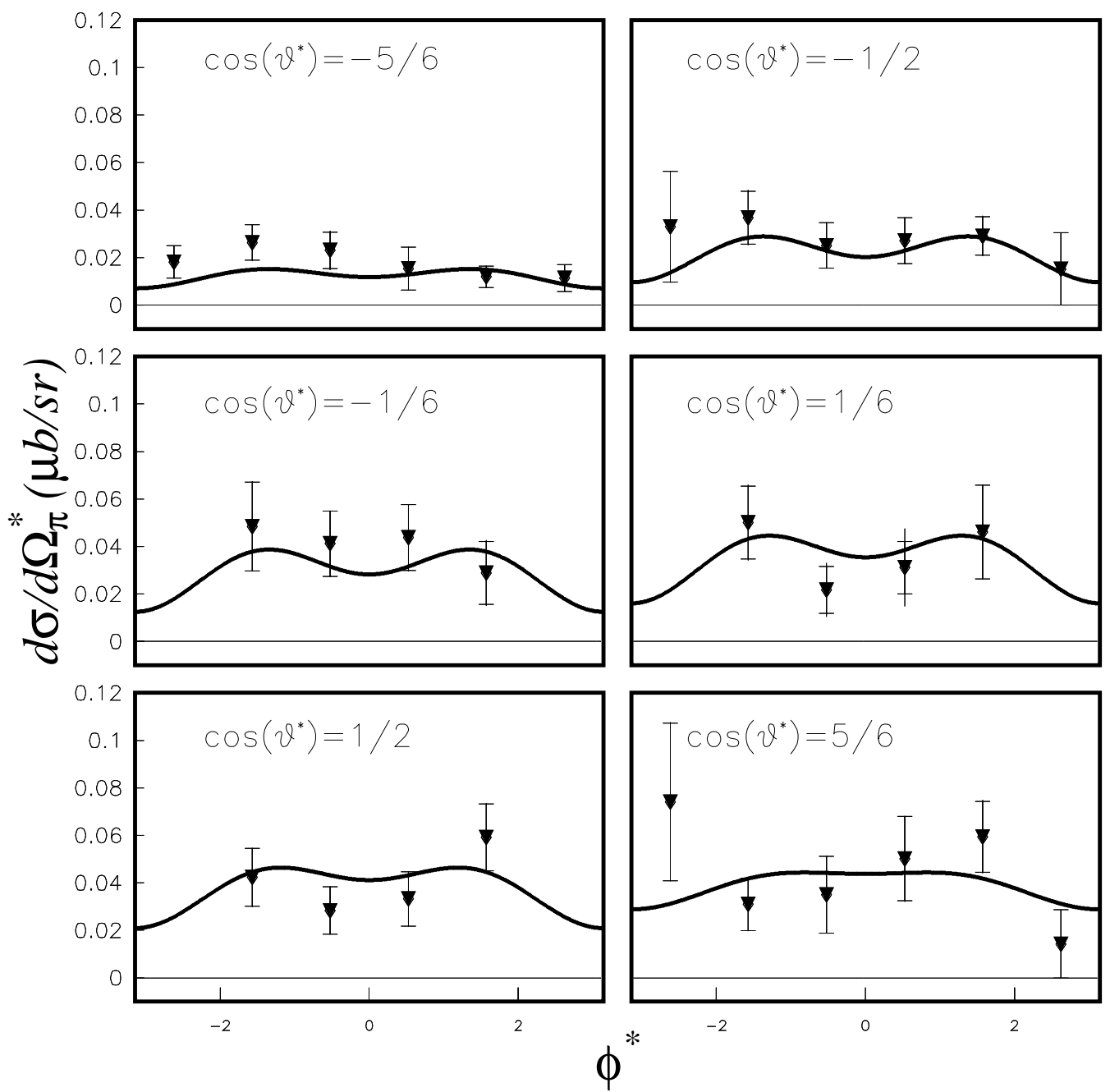

Figure E.24: Center of mass differential cross sections for $W=1.232 \mathrm{GeV}$ and $Q^{2}=7.692 \mathrm{GeV}^{2} \cdot \frac{\chi^{2}}{n_{\text {dof }}}=0.785$. 

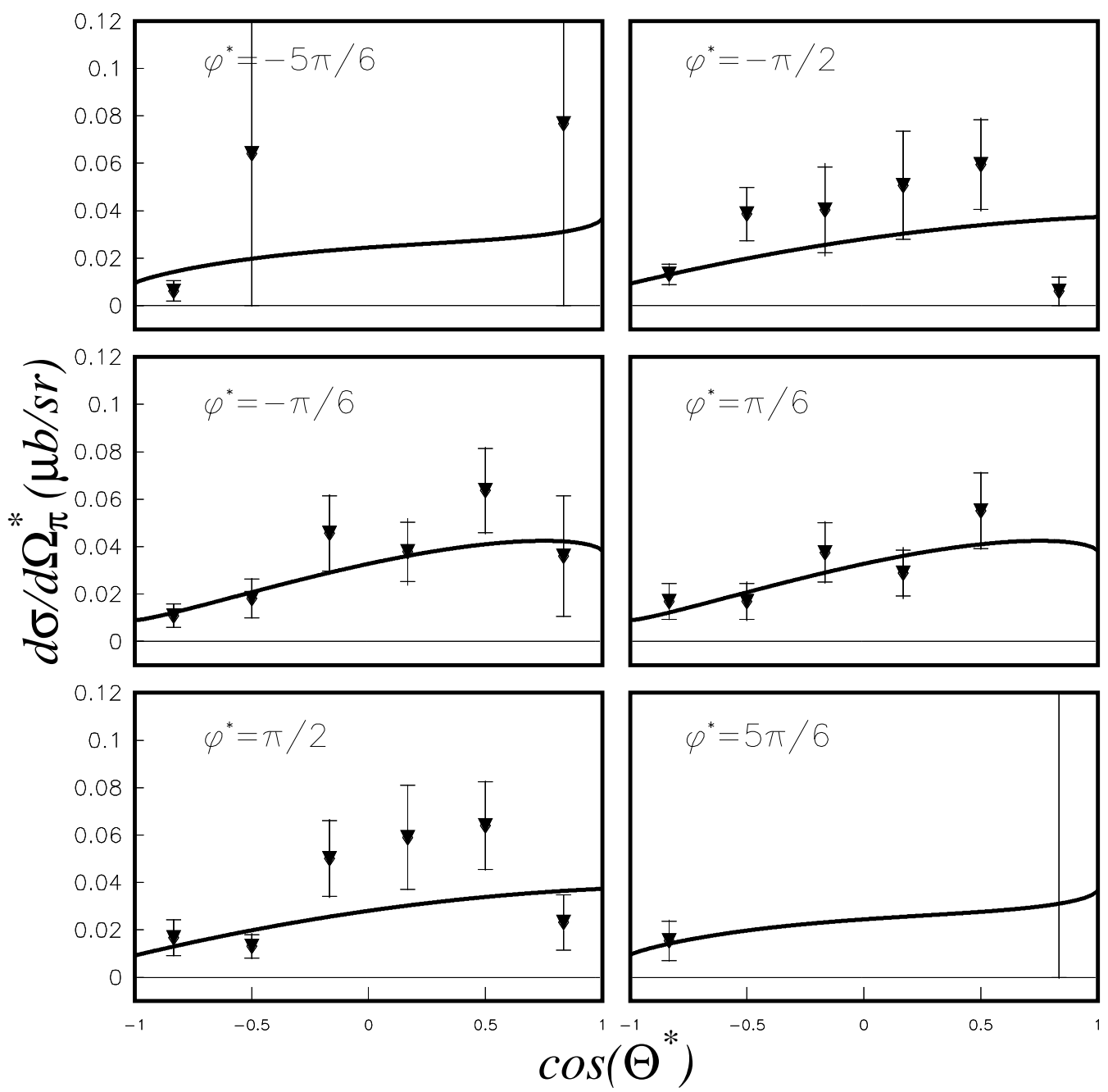

Figure E.25: Center of mass differential cross sections for $W=1.272 \mathrm{GeV}$ and $Q^{2}=7.608 \mathrm{GeV}^{2} \cdot \frac{\chi^{2}}{n_{\text {dof }}}=1.435$. 

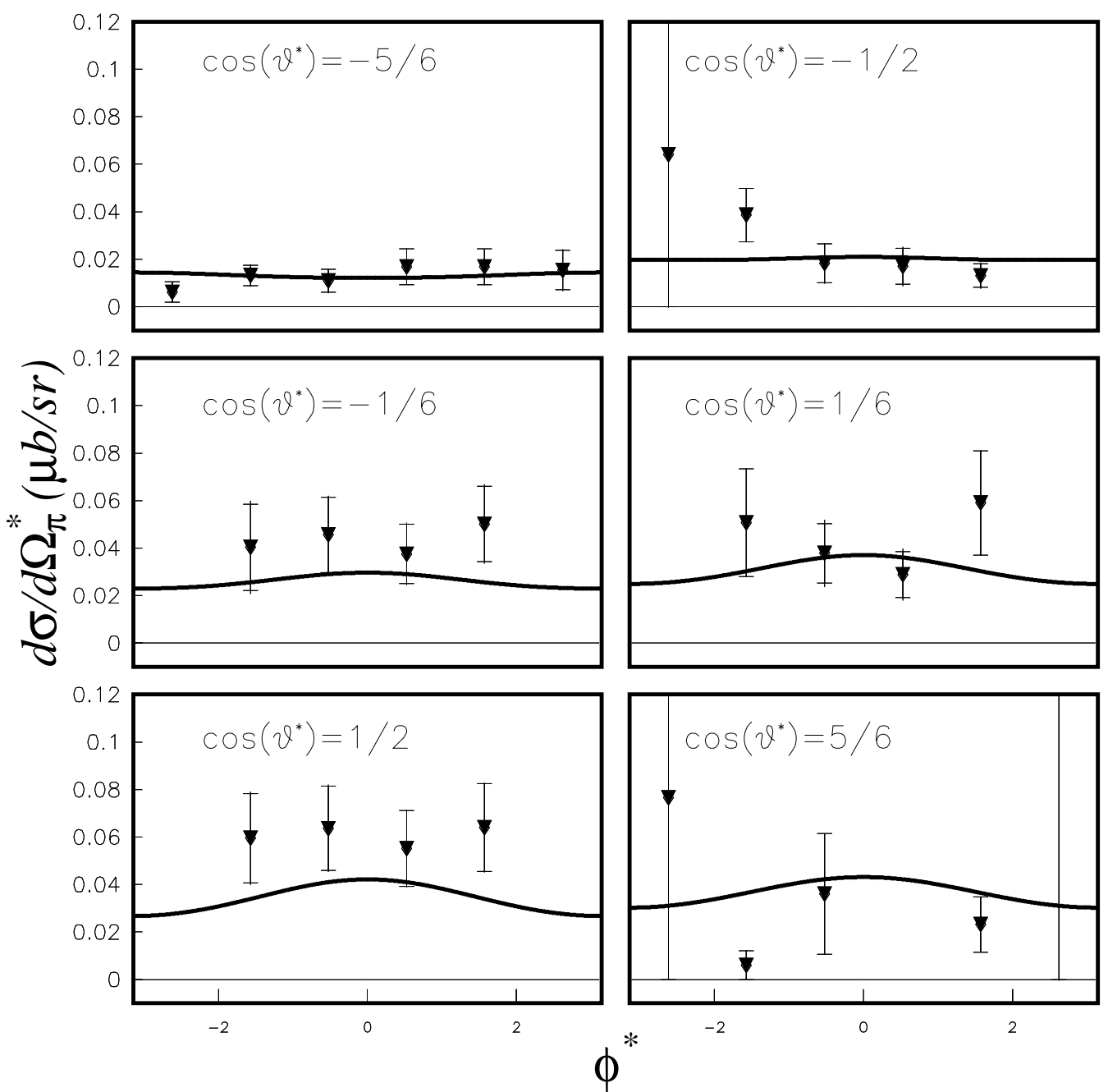

Figure E.26: Center of mass differential cross sections for $W=1.272 \mathrm{GeV}$ and $Q^{2}=7.608 \mathrm{GeV}^{2} \cdot \frac{\chi^{2}}{n_{\text {dof }}}=1.435$. 

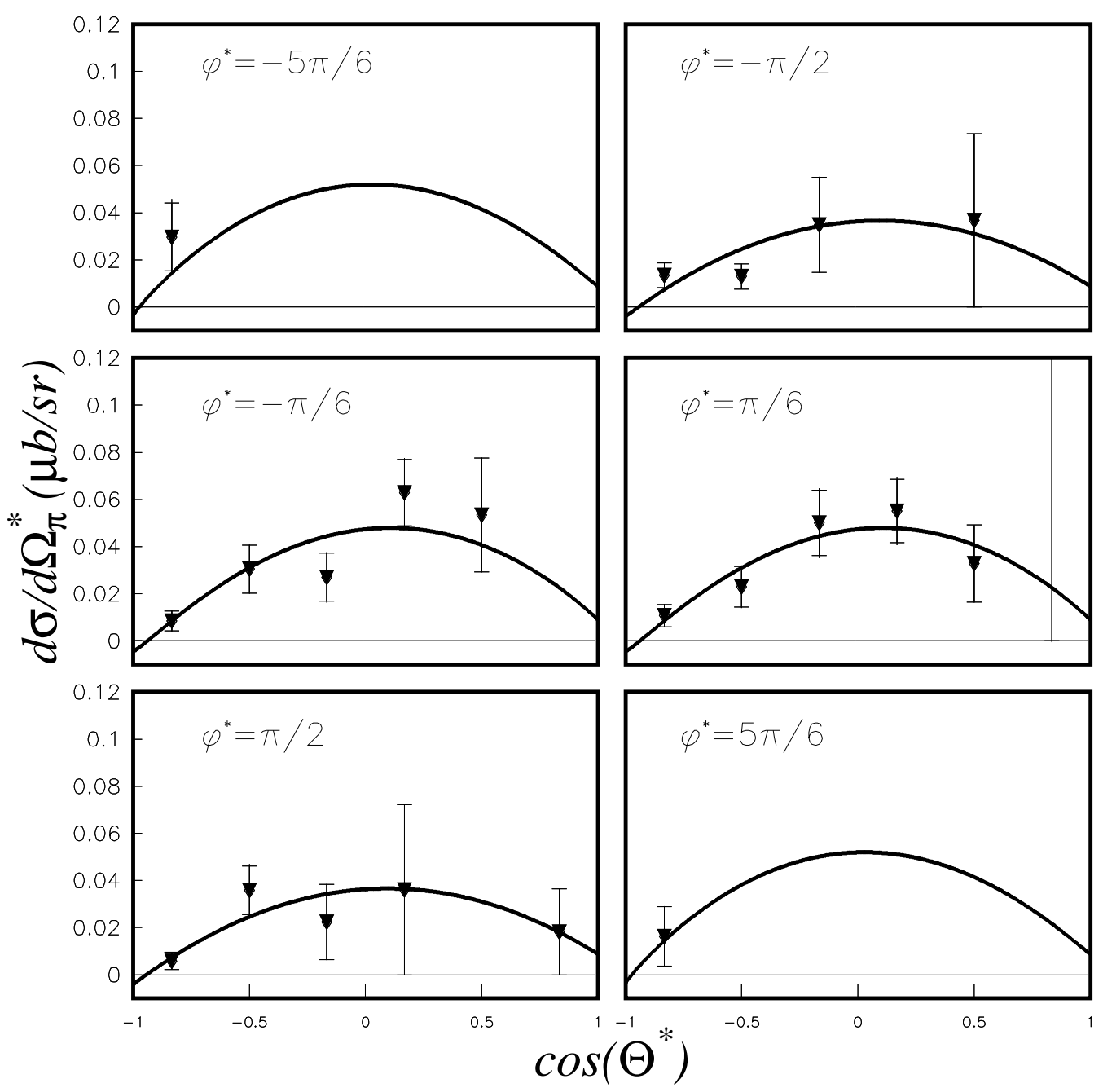

Figure E.27: Center of mass differential cross sections for $W=1.312 \mathrm{GeV}$ and $Q^{2}=7.524 \mathrm{GeV}^{2} \cdot \frac{\chi^{2}}{n_{\text {dof }}}=0.737$. 

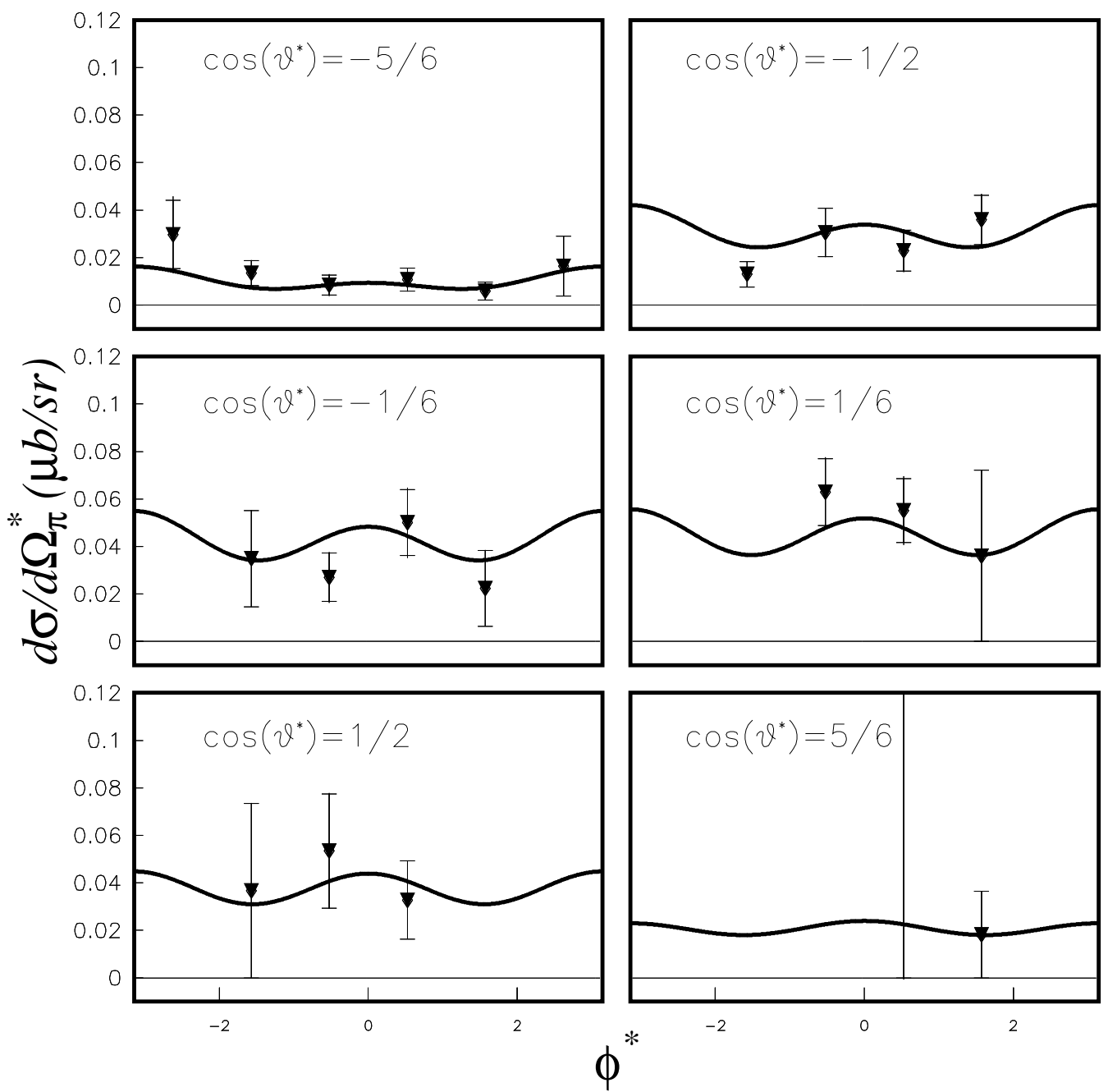

Figure E.28: Center of mass differential cross sections for $W=1.312 \mathrm{GeV}$ and $Q^{2}=7.524 \mathrm{GeV}^{2} \cdot \frac{\chi^{2}}{n_{\text {dof }}}=0.737$. 

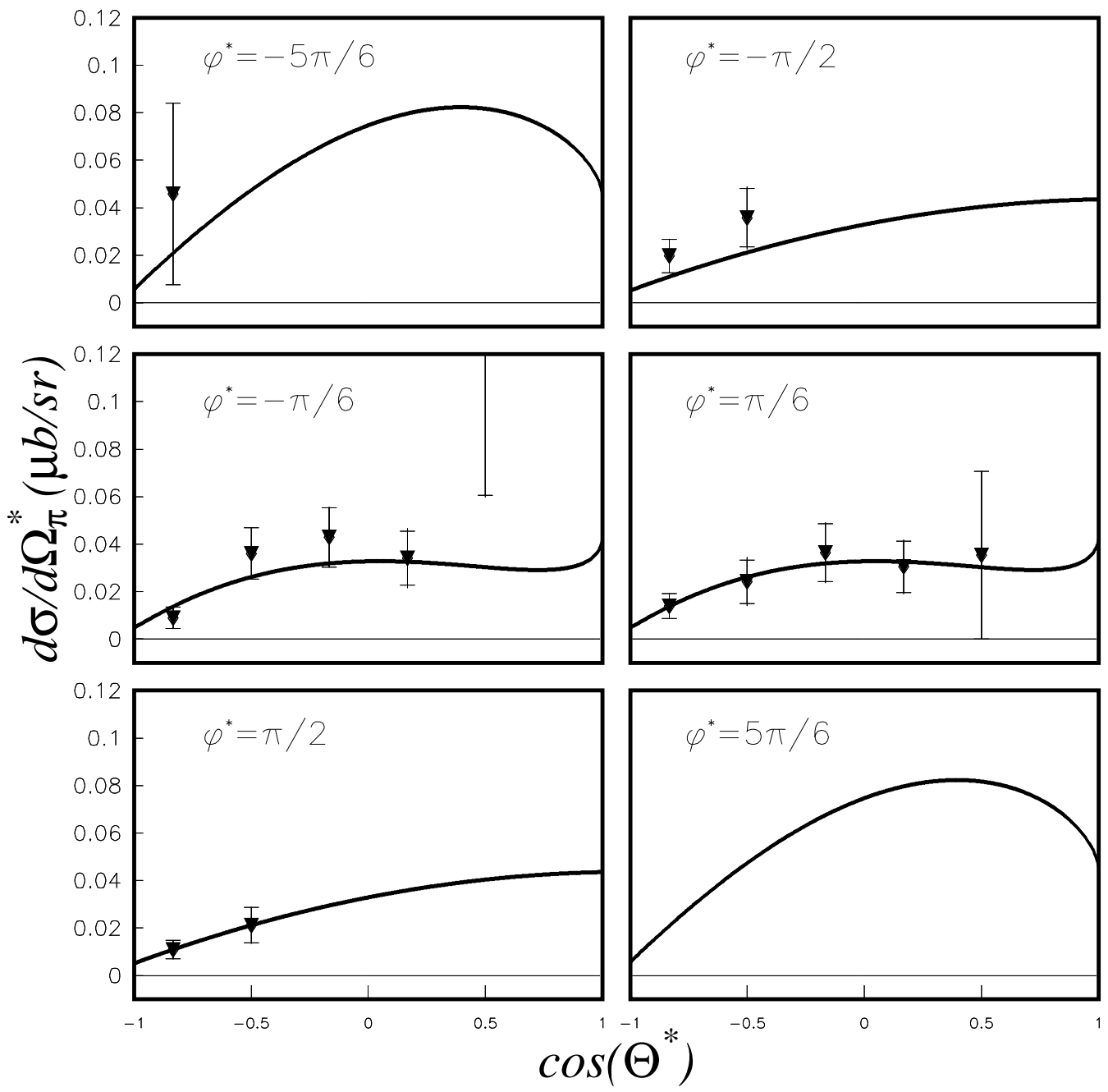

Figure E.29: Center of mass differential cross sections for $W=1.352 \mathrm{GeV}$ and $Q^{2}=7.436 \mathrm{GeV}^{2} \cdot \frac{\chi^{2}}{n_{\text {dof }}}=0.649$. 

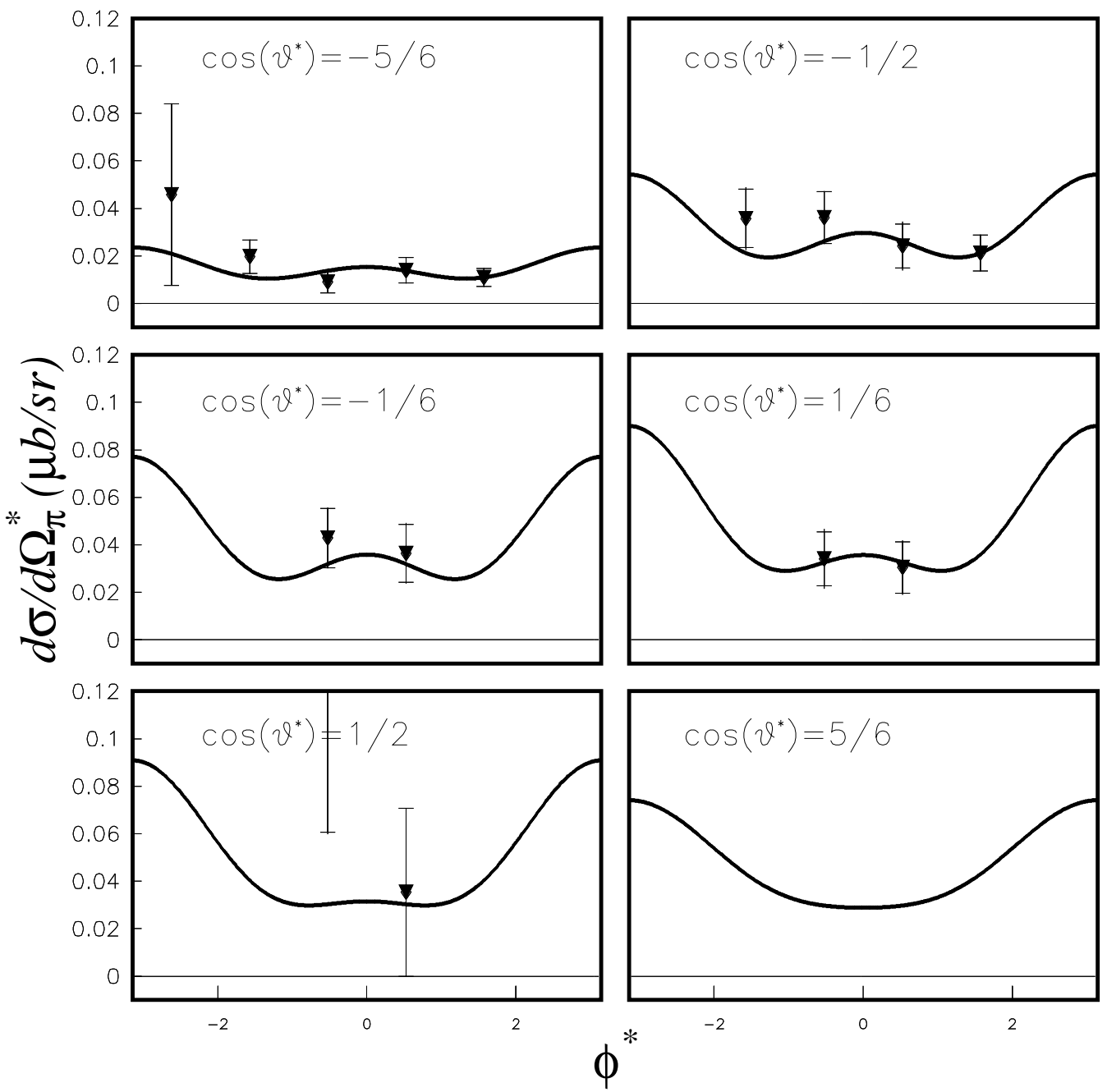

Figure E.30: Center of mass differential cross sections for $W=1.352 \mathrm{GeV}$ and $Q^{2}=7.436 \mathrm{GeV}^{2} \cdot \frac{\chi^{2}}{n_{\text {dof }}}=0.649$. 

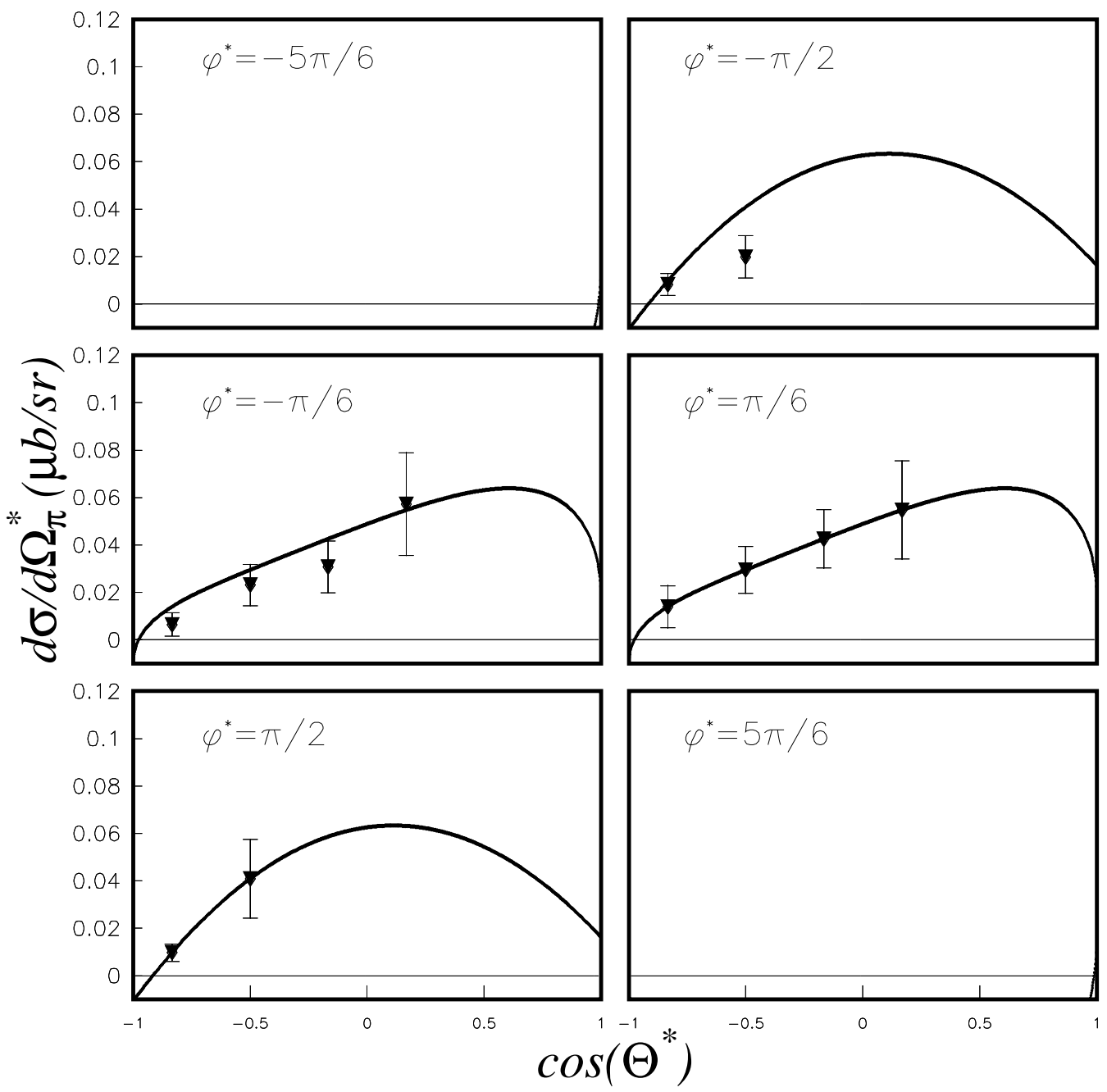

Figure E.31: Center of mass differential cross sections for $W=1.392 \mathrm{GeV}$ and $Q^{2}=7.348 \mathrm{GeV}^{2} \cdot \frac{\chi^{2}}{n_{\text {dof }}}=0.442$. 

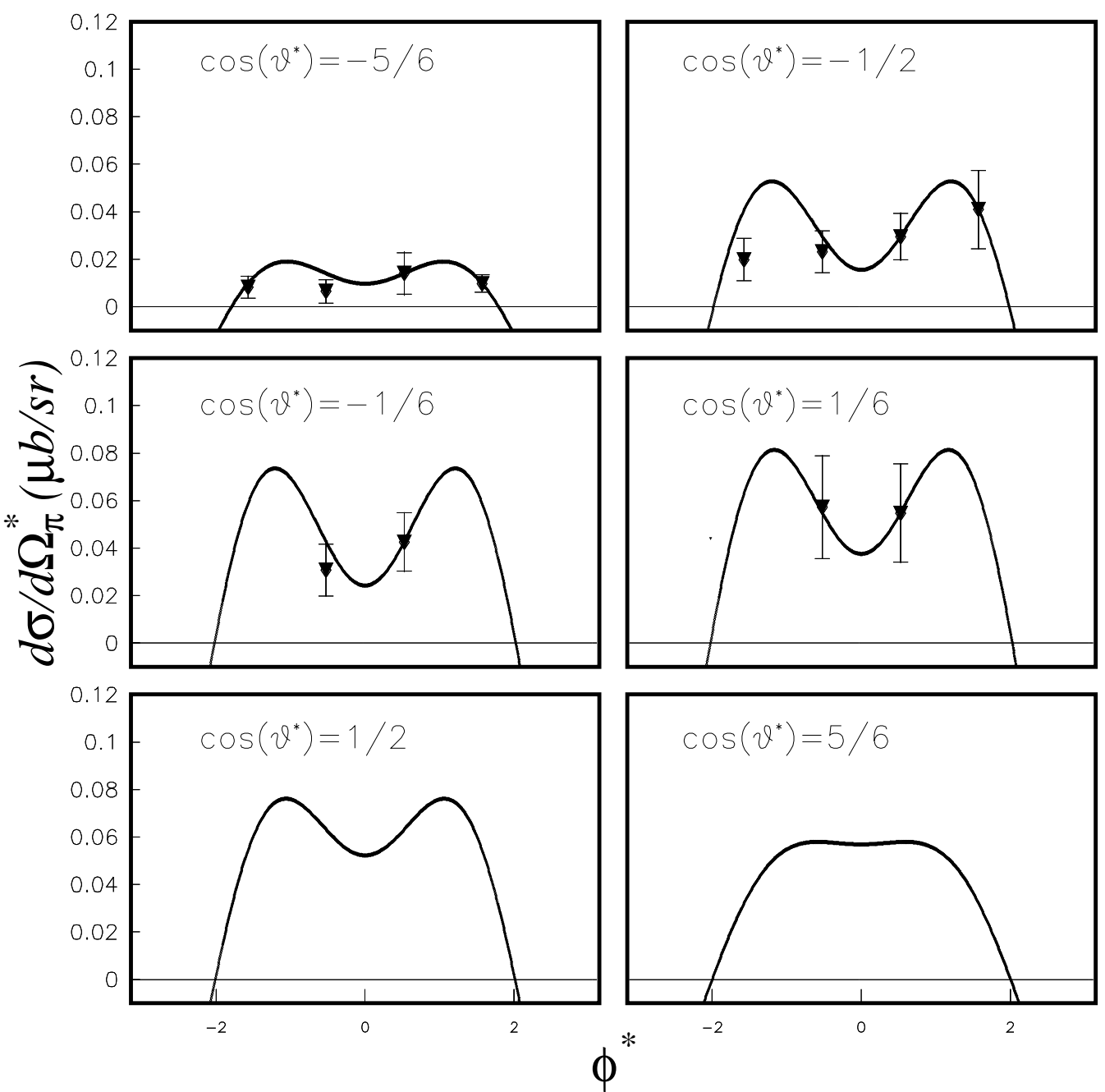

Figure E.32: Center of mass differential cross sections for $W=1.392 \mathrm{GeV}$ and $Q^{2}=7.348 \mathrm{GeV}^{2} \cdot \frac{\chi^{2}}{n_{\text {dof }}}=0.442$. 